\title{
Two Biosynthetic Pathways in Jahnella thaxteri for Thaxteramides, Distinct Types of Lipopeptides
}

Emilia Oueis $^{\dagger \S}$, Thorsten Klefisch ${ }^{\dagger \S}$, Nestor Zaburannyi, ${ }^{\dagger}$ Ronald Garcia $^{\dagger \dagger}$, Alberto Plaza ${ }^{\dagger}$, and Rolf Müller*t'

${ }^{\dagger}$ Department of microbial natural products, Helmholtz-institute for pharmaceutical research Saarland (HIPS), Helmholtz center for infection research (HZI), Campus E8.1, 66123 Saarbrücken, Germany

${ }^{\ddagger}$ German Centre for Infection Research (DZIF), Partner Site Hannover-Braunschweig, Germany

Rolf.Mueller@helmholtz-hips.de 


\section{Table of Contents}

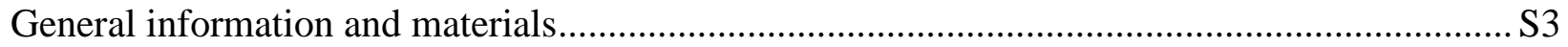

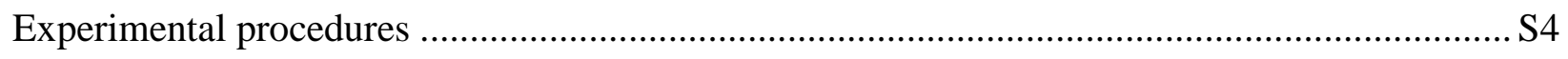

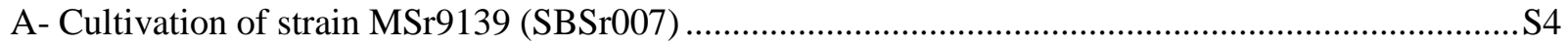

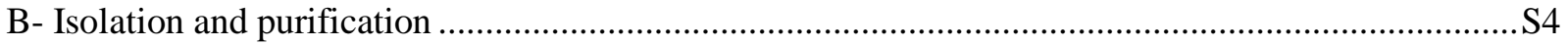

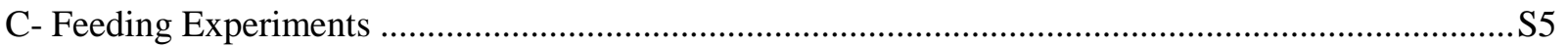

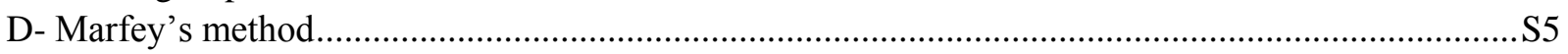

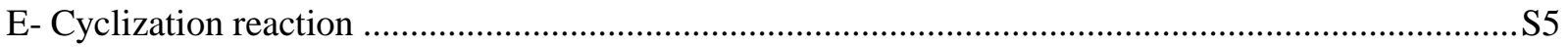

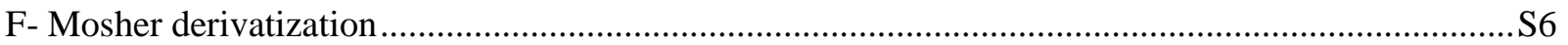

G- Synthesis of $(S)$ - $\beta$-aminoisobutyric acid methyl ester ............................................................. 6

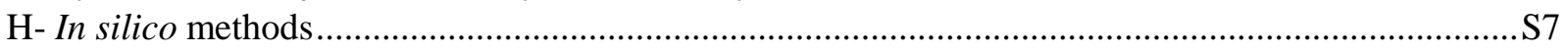

Phylogenetic analysis of $\mathrm{C}$ domains in thaxteramide NRPSs ....................................... 77

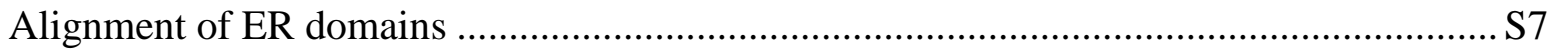

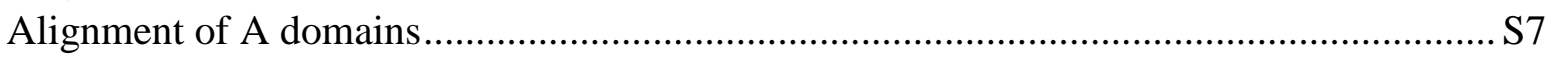

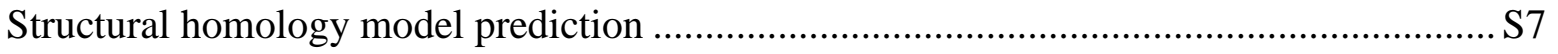

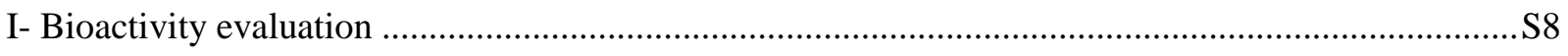

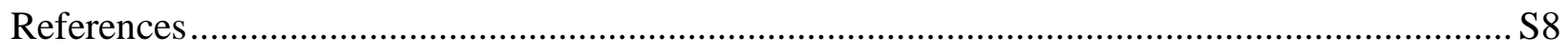

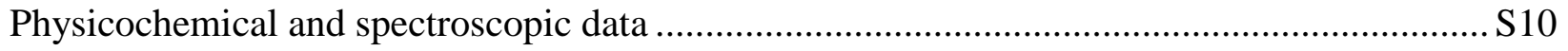

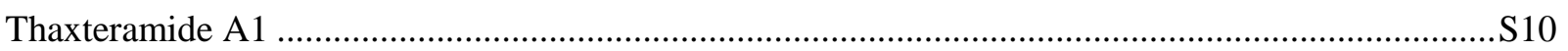

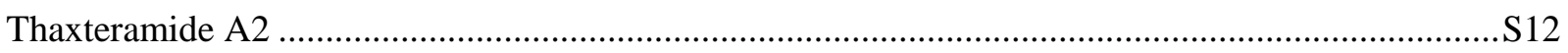

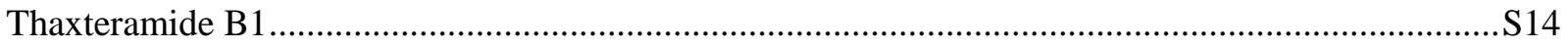

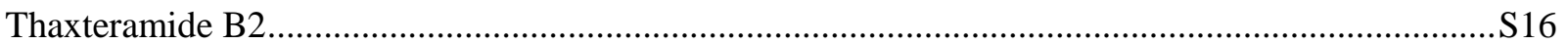

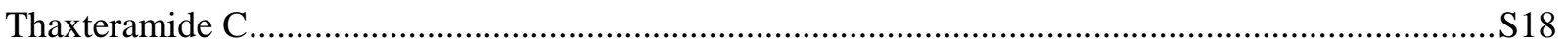

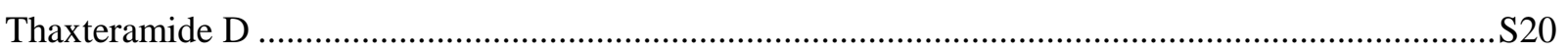

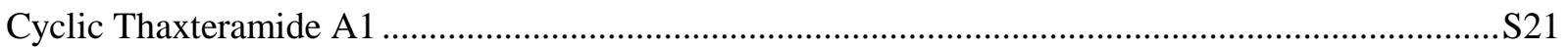

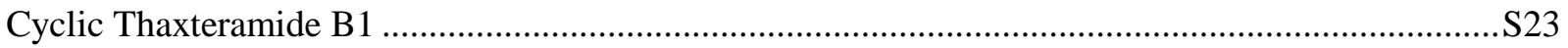

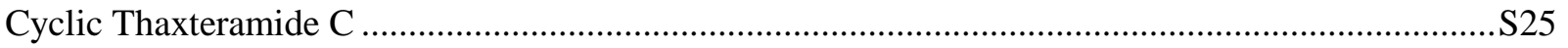

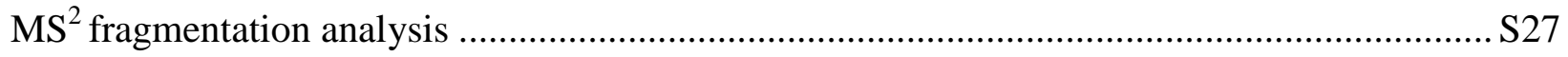

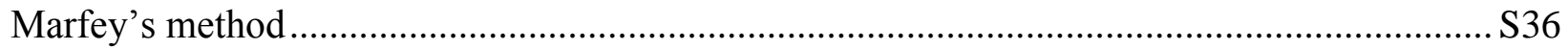

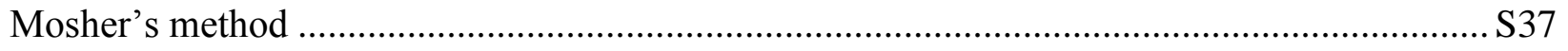

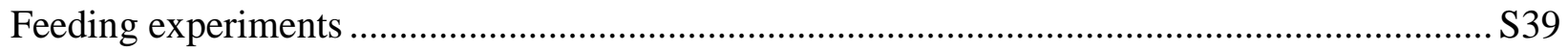

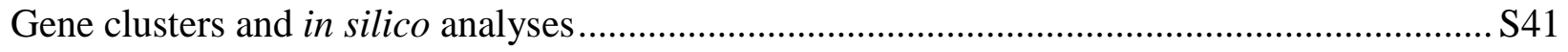

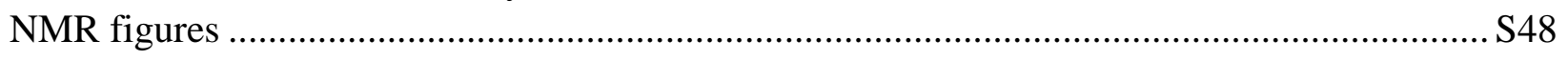

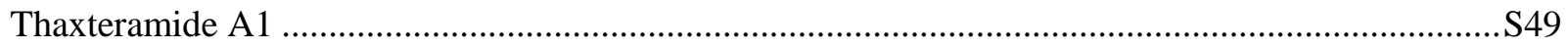

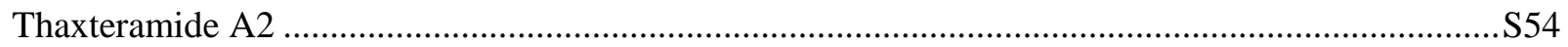

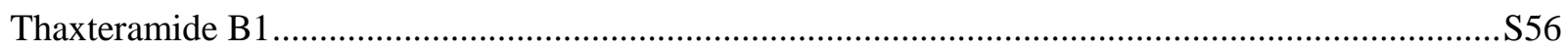

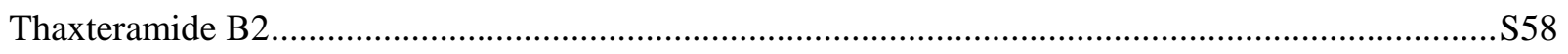

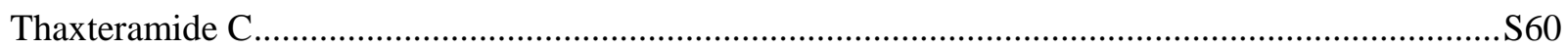

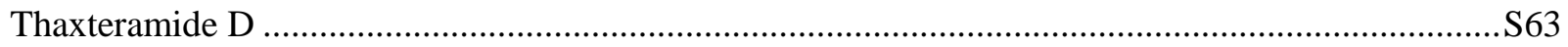

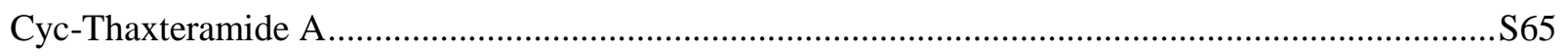

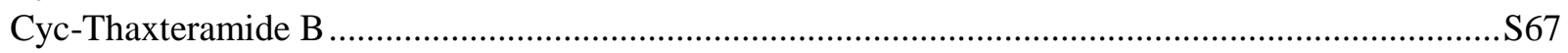

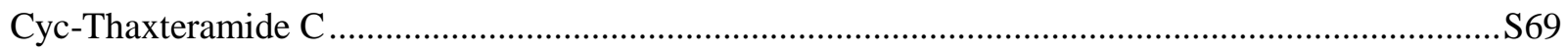

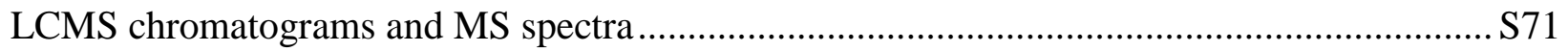




\section{General information and materials}

Aqueous mobile-phases for semi-preparative HPLC were prepared using water purified with a Milli- $\mathrm{Q}^{\circledR}$ Integral water purification system (purified to a resistivity of $18.2 \mathrm{M} \Omega . \mathrm{cm}$ at $25^{\circ} \mathrm{C}$ ). $\mathrm{MeCN}$ and formic acid used were HPLC grade. Mobile-phases for analytical HPLC were prepared using water distilled over a vertical double distillation apparatus, LC-MS grade organic solvents further distilled, and LC-MS grade formic acid. Other solvents, compounds, and reagents were purchased commercially from different sources and used without any further purification.

NMR spectra $(1 \mathrm{H}, 2 \mathrm{D})$ were recorded on a Bruker Ascend 700 spectrometer with a $5 \mathrm{~mm}$ TXI cryoprobe $\left({ }^{1} \mathrm{H}\right.$ at $700 \mathrm{MHz},{ }^{13} \mathrm{C}$ at $\left.175 \mathrm{MHz}\right)$ at ambient temperature using standard pulse programs. Chemical shifts are expressed in parts per million (ppm) from $\mathrm{CD}_{3} \mathrm{OD}(\delta \mathrm{H}=3.31, \delta \mathrm{C}$ = 49.0) ${ }^{1}$ Multiplicities are described as s (singlet), d (doublet), q (quadruplet), dd (doublet of doublets), ddd (doublet of doublets of doublets), $t$ (triplet), dd (doublet of triplets), m (multiplet), br (broad). Coupling constants $J$ are quoted in Hertz (Hz) to the nearest $0.1 \mathrm{~Hz}$.

Analytical LC-MS was performed on a Dionex Ultimate 3000 UPLC system (Thermo Fisher) using a Waters BEH C18 $(100 \times 2.1 \mathrm{~mm}, 1.7 \mu \mathrm{m})$, equipped with a DAD module, and coupled to a Bruker maXis 4G UHR-TOF mass spectrometer (Bruker Daltonics) with electrospray ionization (ESI) and $\mathrm{MS}^{2}$ capabilities. High resolution mass spectra (HRMS) and $\mathrm{MS}^{2}$ fragmentation spectra were recorded using the same systems as for LC-MS. The following chromatographic systems were used: System A: at $45^{\circ} \mathrm{C}$ and a flow rate of $0.6 \mathrm{~mL} / \mathrm{min}$ with $\mathrm{MeCN} / 0.1 \% \mathrm{FA}$ and $\mathrm{H}_{2} \mathrm{O} / 0.1 \% \mathrm{FA}$ [ $5 \% \mathrm{MeCN}$ ( $\left.0.5 \mathrm{~min}\right)$, linear gradient from 5 to $95 \%$ of $\mathrm{MeCN}$ (18 $\mathrm{min}$ ), 95\% MeCN (2 min) followed by re-equilibration to starting conditions] and UV detection in the range from 200 to $600 \mathrm{~nm}$. System B: at $45^{\circ} \mathrm{C}$ and a flow rate of $0.6 \mathrm{~mL} / \mathrm{min}$ with $\mathrm{MeCN} / 0.1 \%$ FA and $\mathrm{H}_{2} \mathrm{O} / 0.1 \%$ FA [5 to $10 \%$ of $\mathrm{MeCN}$ ( $1 \mathrm{~min}$ ), 10 to 35\% of MeCN (14 $\mathrm{min}$ ), 35 to $55 \% \mathrm{MeCN}$ (7 min), 55-80\% MeCN (3 min), 80\% MeCN (1 min) followed by reequilibration to starting conditions] and UV detection in the range from 200 to $600 \mathrm{~nm}$. The LC flow was split to $75 \mu \mathrm{L} / \mathrm{min}$ before entering the maXis $4 \mathrm{G}$ mass spectrometer using the standard ESI source. Mass spectra were acquired in centroid mode ranging from 150-2000 m/z at $2 \mathrm{~Hz}$ scan speed with automatic $\mathrm{MS}^{2}$ settings (system A) or 150-1000 m/z (system B).

Preparative RP-HPLC was performed on a Waters Autopurifier system (Waters) equipped with a DAD detector module and a single-quad MS spectrometer using a waters XBridge C18 column $(10 \mu \mathrm{m}, 19 \times 150 \mathrm{~mm})$ at $25 \mathrm{~mL} / \mathrm{min}$ flow rate and fractions were collected automatically by time-based collection and their purity verified by analytical LC-MS. The chromatographic system $\mathbf{P}$ used was as follows: $\mathrm{MeCN} / 0.1 \% \mathrm{FA}$ and $\mathrm{H}_{2} \mathrm{O} / 0.1 \%$ FA [20\% $\mathrm{MeCN}$ (5 min), 20 to $27.5 \%$ of $\mathrm{MeCN}$ ( $25 \mathrm{~min}$ ), 27.5 to $95 \%$ of $\mathrm{MeCN}$ (1 min),], UV and MS detection.

Semi-preparative RP-HPLC was performed on a Dionex Ultimate (Thermo Fisher) equipped with a DAD detector and for some a ISQEM single-quad MS spectrometer using either a Waters

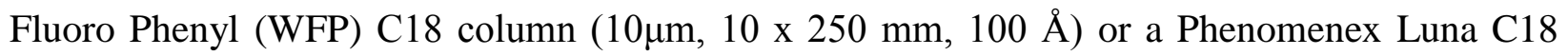


(PLC) column $(10 \mu \mathrm{m}, 10 \times 250 \mathrm{~mm}, 100 \AA)$ at $40^{\circ} \mathrm{C}$ and $5 \mathrm{~mL} / \mathrm{min}$ flow rate and fractions were collected automatically by time-based collection and their purity verified by analytical LC-MS. Multiple chromatographic systems were used; System SP1: WFP; MeCN / 0.1\% FA and $\mathrm{H}_{2} \mathrm{O} /$ $0.1 \% \mathrm{FA}$ [5\% MeCN (3 $\mathrm{min}), 5$ to $25 \%$ of $\mathrm{MeCN}$ (20 $\mathrm{min}$ ), 25 to $25.5 \% \mathrm{MeCN}$ (5min)] and UV detection at 190, 220, 254, and $280 \mathrm{~nm}$. System SP2: WFP; MeCN / 0.1\% FA and $\mathrm{H}_{2} \mathrm{O} / 0.1 \%$ FA [5\% MeCN (3 min), 5 to $15 \%$ of $\mathrm{MeCN}$ (10 $\mathrm{min}), 15$ to $16.7 \% \mathrm{MeCN}(17 \mathrm{~min})]$ and UV detection at 190, 220, 254, and $280 \mathrm{~nm}$. System SP3: PLC; MeCN / 0.1\% FA and $\mathrm{H}_{2} \mathrm{O} / 0.1 \%$ FA [5\% MeCN (5 min), 5 to $50 \%$ of $\mathrm{MeCN}$ (10 min), 50\% $\mathrm{MeCN}(5 \mathrm{~min})]$ and UV detection at 190, 220, 254, and $280 \mathrm{~nm}$. SP4: PLC; MeCN / 0.1\% FA and $\mathrm{H}_{2} \mathrm{O} / 0.1 \%$ FA [0 to $10 \%$ of $\operatorname{MeCN}(16 \mathrm{~min})]$ and UV detection at 190, 220, 254, and $280 \mathrm{~nm}$.

LCMS and $\mathrm{MS}^{2}$ fragmentation data were visualised and analysed using Bruker DataAnalysis software. The software was also used as an LCMS-based de-replication tool by comparison of the LCMS data (mass and rt) of peaks detected within crude extracts to an in house database of known myxobacterial compounds.

Bioassays were conducted on pure compounds to test for cytotoxic, antimicrobial, and antifungal activity. Cytotoxic assays were conducted against KB3.1 (human papillomavirus-related endocervical adenocarcinoma) cell line, antimicrobial tests against a range of Gram-positive and Gram-negative bacteria and antifungal tests against some fungi and yeast. Detailed strain information is given below in experimental procedures, section I. All microorganisms were obtained from the German Collection of Microorganisms and Cell Cultures (Deutsche Sammlung für Mikroorganismen und Zellkulturen, DSMZ) or were part of our internal strain collection.

\section{Experimental procedures}

\section{A- Cultivation of strain MSr9139 (SBSr007)}

The myxobacterium Jahnella thaxteri MSr9139 (SBSr007) was isolated from a dried soil sample with decayed plant materials using mineral salt agar and was maintained by cultivating in buffered VY/2 agar. $^{2-3}$ Fermentation was performed in $8 \times 2$ L flasks containing $1 \mathrm{~L}$ buffered VY/2 broth. ${ }^{3}$ The flask cultures were grown for $10 \mathrm{~d}$ with replacement of the medium every $24 \mathrm{~h}$.

\section{B- Isolation and purification}

The XAD-16 resin (5\%) was added to cultures two days before harvesting. The cell pellet and $\mathrm{XAD}$ resin were then collected by decantation and were extracted three times with methanol. The evaporated combined fractions yielded an orange crude extract, which was analysed by LCMS. Subsequently, the extract was fractionated by preparative HPLC according to system $\mathbf{P}$ and each fraction enriched with one derivative was further purified by semi-preparative HPLC using either system SP1 or SP2. 


\section{C- Feeding Experiments}

Cultivation of MSr9139 was performed in $25 \mathrm{~mL}$ buffered VY/2 broth containing $1 \mathrm{~mL}$ XAD-16 resin. The cultures were grown for $7 \mathrm{~d}$ at $30^{\circ} \mathrm{C}$. Labelled precursors were dissolved in either water or DMSO at 1 or $2 \mathrm{M}$ concentration, and $50 \mu \mathrm{L}$ of each were added to individual cultures over five days to a final concentration of 1 or $2 \mathrm{mM}$, respectively. The individual cell pellets and XAD resin were harvested on day 7 and extracted with $2 \times 25 \mathrm{~mL}$ methanol. The organic solvent was evaporated and the crude extract dissolved in $500 \mu \mathrm{L}$ methanol and analysed by HR-ESIMS.

\section{D- Marfey's method}

50-100 $\mu \mathrm{g}$ of each peptide were dissolved in $100 \mu \mathrm{L}$ of $6 \mathrm{M} \mathrm{HCl}$. The reaction was stirred at 110 ${ }^{\circ} \mathrm{C}$ for 45 minutes, and then dried at $110{ }^{\circ} \mathrm{C}$ in 15 minutes. Each sample was reacted separately with $50 \mu \mathrm{L}$ of L-FDLA and D-FDLA (1\% (w/v) Marfey's reagent in acetone) and $20 \mu \mathrm{L}$ of $1 \mathrm{M}$ $\mathrm{NaHCO}_{3}$ for 2 hours at $40{ }^{\circ} \mathrm{C}, 700 \mathrm{rpm}$. To quench the reaction, $10 \mu \mathrm{L}$ of $2 \mathrm{M} \mathrm{HCl}$ were added, and each sample was diluted with $300 \mu \mathrm{L} \mathrm{MeCN}$. The samples were centrifuged and the supernatant was measured by LC-MS using system $\mathbf{B}$. The same sequence was performed with standard and synthetic amino acids.

\section{E- Cyclization reaction}

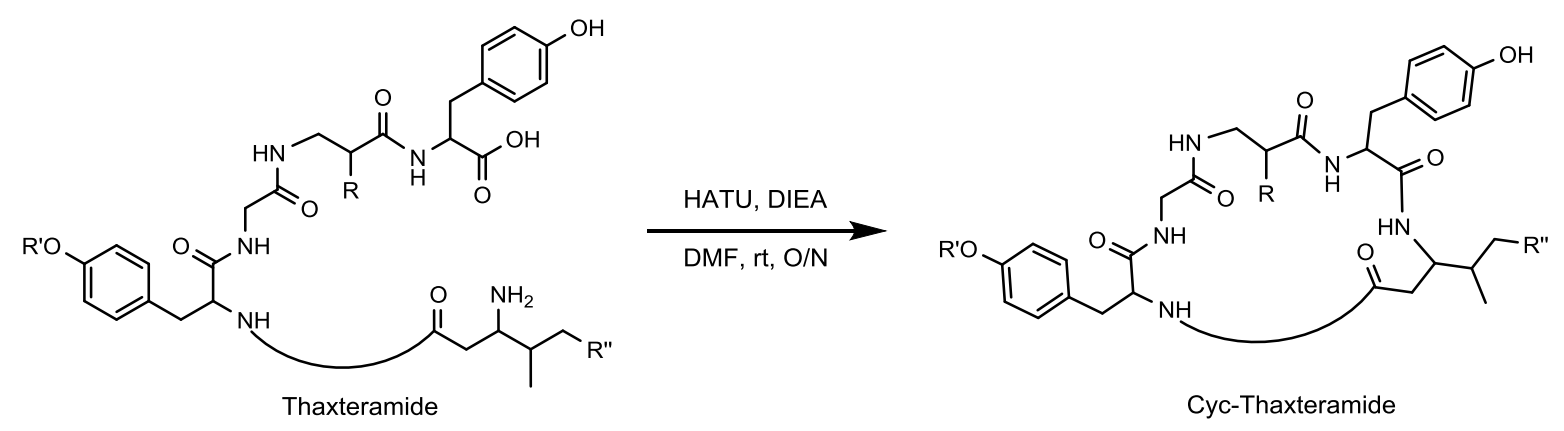

Scheme S1: Cyclization reaction of thaxteramide derivatives

To a solution of the linear lipopeptide in DMF was added 1.5 equivalents of HATU followed by 2.5 equivalents of DIEA. The reaction was stirred at room temperature overnight under inert atmosphere. Upon completion of the reaction (LC-MS monitoring), the reaction mixture was purified by semi-preparative HPLC using system SP3. The corresponding fractions were collected, evaporated under reduced vacuum to a few $\mathrm{mLs}$, and the remaining dried using a vacuum concentrator. 


\section{F- Mosher derivatization}

To a solution of ThxA1 in anhydrous THF was added four equivalents of DIEA followed by 15 equivalents of $(R)$ - or $(S)$-MTPA-Cl ( $\alpha$-methoxy- $\alpha$-(trifluoromethyl)phenylacetyl chloride or Mosher chloride), and the reaction left stirring at room temperature under inert atmosphere until completion. The reaction was monitored by LC-MS. A solution of aqueous HCL (0.1 M) was added to the reaction and the crude mixture freeze-dried. The resulting crude powder was used without any further purification for NMR. The major compound of both reactions was that resulting from one addition of the Mosher chloride onto the free amine, affording the $(S)$-amide (from $(R)$-chloride) and the $(R)$-amide (from $(S)$-chloride).

\section{G- Synthesis of $(S)$ - $\beta$-aminoisobutyric acid methyl ester ${ }^{4}$}

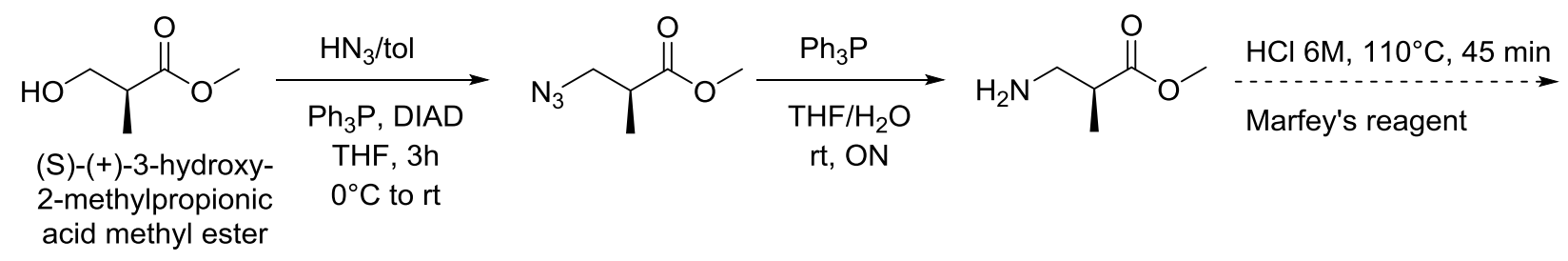

Scheme S2: Synthesis of $(S)-\beta$-aminoisobutyric acid methyl ester standard for Marfey's analysis

To a solution of triphenylphosphine $\left(\mathrm{Ph}_{3} \mathrm{P}, 0.5 \mathrm{~g}, 2 \mathrm{mmol}\right)$ and diisopropyl azodicarboxylate (DIAD, $390 \mu \mathrm{L}, 2 \mathrm{mmol})$ in anhydrous THF $(5.2 \mathrm{~mL})$ at $0^{\circ} \mathrm{C}$ under inert atmosphere, a solution of hydrazoic acid in toluene $(0.5 \mathrm{M}, 4.2 \mathrm{~mL}, 2.1 \mathrm{mmol})$ was added drop-wise. A solution of (S)(+)-3-hydroxy-2-methylpropionic acid methyl ester $(100 \mu \mathrm{L}, 0.9 \mathrm{mmol})$ solubilised in anhydrous THF $(0.5 \mathrm{~mL})$ was added to the mixture and the reaction left stirring at room temperature for $3 \mathrm{~h}$. Upon completion of the reaction (monitoring by TLC), the solvent was concentrated under reduced pressure and the crude mixture was used for the next step without any further purification.

The crude azide mixture $(0.9 \mathrm{mmol})$ and $\mathrm{Ph}_{3} \mathrm{P}(0.26 \mathrm{~g}, 1 \mathrm{mmol})$ were solubilised in THF $(8.3$ $\mathrm{mL})$ and $\mathrm{H}_{2} \mathrm{O}(2.1 \mathrm{~mL})(4 / 1, \mathrm{v} / \mathrm{v})$ and the reaction stirred at room temperature overnight. The THF was evaporated under reduced pressure and the water phase was acidified with $\mathrm{HCl} 1 \mathrm{M}$ to $\mathrm{pH}=1$. The aqueous phase was washed three times with EtOAc to eliminate triphenylphosphine oxide $\left(\mathrm{Ph}_{3} \mathrm{PO}\right)$, the side product of both previous reactions. The aqueous phase was evaporated to dryness, affording the desired $(S)$ - $\beta$-aminoisobutyric acid methyl ester as a light brown solid.

$(S)$ - $\beta$-aminoisobutyric acid methyl ester was used as is without any further purification as a Marfey's standard. 


\section{H- In silico methods}

The Jahnella thaxteri MSr9139 gene cluster sequences for thaxteramide A/B and thaxteramide C biosynthesis were deposited in GenBank with the accession numbers MK551162 and MK551161, respectively.

Genomic DNA of Jahnella thaxteri MSr9139 was sequenced by Illumina sequencing technology. ${ }^{5}$ The prediction and analysis of thaxteramides biosynthesis gene clusters was done using antiSMASH 3.0. ${ }^{6}$ The functional prediction of open reading frames (ORFs) encoding proteins was performed using protein blast and/or blastx program and Pfam. ${ }^{7}$

\section{Phylogenetic analysis of $C$ domains in thaxteramide NRPSs}

For phylogenetic analysis, amino acid sequences of $525 \mathrm{C}$ domains of known NRPSs ${ }^{8}$ were aligned using the Geneious Alignment tool in Geneious Prime 2019 with default settings (Global alignment with free end gaps, cost matrix: Blosum62, gap open penalty: 12, gap extension penalty: 3 , refinement iterations: 2). The maximum likelihood phylogenetic tree was constructed using FastTree 2.1.5 using default settings.

\section{Alignment of ER domains}

Amino acid sequences of 29 ER domains (20 from Kwan et al. ${ }^{9}$ ) of known PKSs were aligned using the Geneious Alignment tool in Geneious Prime 2019 with default settings (Global alignment with free end gaps, cost matrix: Blosum62, gap open penalty: 12, gap extension penalty: 3 , refinement iterations: 2 ).

\section{Alignment of A domains}

Amino acid sequences of known A domains from NRPS genes, of which the specificity has been determined to be for alanine or other $\beta$-amino acids, were aligned using the Geneious Alignement tool in Geneious Prime 2019 with default settings (Global alignment with free end gaps, cost matrix: Blosum62, gap open penalty: 12, gap extension penalty: 3, refinement iterations: 2). These $A$ domains were collected using the MiBIG repository website. ${ }^{10}$

\section{Structural homology model prediction}

The amino acid sequence of the MT domain was analysed using the Phyre2 web portal for protein modelling, prediction and analysis. ${ }^{11}$ It consists of advanced remote homology detection methods that build 3D models to predict ligand binding sites and analyse the effect of amino-acid variants within a custom protein sequence. 


\section{I- Bioactivity evaluation}

Aliquots $(20 \mu \mathrm{L}, 1 \mathrm{mg} / \mathrm{mL})$ of ThxA1, ThxA2, ThxB1, ThxB2, ThxC, ThxD, cyc-ThxA, and cyc-ThxC were tested against three Gram-positive bacteria (Bacillus subtilis DSM10, Mycobacterium smegmatis mc2155, Staphylococcus aureus Newman), four Gram-negative bacteria (Acinetobacter baumanii DSM30008, Citrobacter freundii DSM30039, Escherichia coli BW25113, Escherichia coli JW0451-2 (acrB), Pseudomonas aeruginosa PA-14), two yeasts (Candida. albicans DSM1665, Pichia anomala DSM6766) and one fungus (Mucor hiemalis DSM2656) with methanol as negative control. The MIC values were determined by serial dilution $(150 \mu \mathrm{L})$ in 96-well plates for tissue cultures (TPP). Overnight cultures were prepared from cryopreserved cultures and were diluted to achieve a final inoculum of $10^{4}-10^{5} \mathrm{cfu} / \mathrm{mL}$. The cell suspension was added and microorganisms were grown for $18-24 \mathrm{~h}$ at $37^{\circ} \mathrm{C}$. Streptococci and enterococci were grown under microaerophilic conditions. Growth inhibition was assessed by visual inspection and given MIC values are the lowest concentration of antibiotic at which there was no visible growth.

A second round of antibacterial evaluation of cyc-ThxA, and cyc-ThxC was conducted against the following Gram-positive bacteria: Streptococcus pneumoniae DSM-20566, Streptococcus pneumoniae DSM-11865 (PRSP), Enterococcus faecium DSM-20477, Enterococcus faecalis DSM-12956 (VRE), Enterococcus faecium 17050 (VRE), Staphylococcus aureus HG001 Dap (DRSA), Staphylococcus aureus HG001, Staphylococcus aureus N315 (MRSA).

\section{References}

[1] G. R. Fulmer, A. J. M. Miller, N. H. Sherden, H. E. Gottlieb, A. Nudelman, B. M. Stoltz, J. E. Bercaw, K. I. Goldberg, Organometallics 2010, 29, 2176-2179.

[2] a) A. Plaza, K. Viehrig, R. Garcia, R. Müller, Org. Lett. 2013, 15, 5882-5885; b) T. Hoffmann, S. Müller, S. Nadmid, R. Garcia, R. Müller, J. Am. Chem. Soc. 2013, 135, 16904-16911.

[3] E. Oueis, H. Stevenson, M. Jaspars, N. J. Westwood, J. H. Naismith, Chem. Commun. 2017, 53, 12274-12277.

[4] B. Simon, Pharmacogenomics 2004, 5, 433-438.

[5] K. Blin, T. Weber, H. U. Kim, S. Y. Lee, M. H. Medema, S. Duddela, D. Krug, R. Müller, R. Bruccoleri, M. A. Fischbach, W. Wohlleben, E. Takano, R. Breitling, Nucleic Acids Res. 2015, 43, W237-W243.

[6] R. D. Finn, P. Coggill, R. Y. Eberhardt, S. R. Eddy, J. Mistry, A. L. Mitchell, S. C. Potter, M. Punta, M. Qureshi, A. Sangrador-Vegas, G. A. Salazar, J. Tate, A. Bateman, Nucleic Acids Res. 2016, 44, D279-D285. 
[7] C. Rausch, I. Hoof, T. Weber, W. Wohlleben, D. H. Huson, BMC Evol. Biol. 2007, 7, 7878.

[8] D. H. Kwan, Y. Sun, F. Schulz, H. Hong, B. Popovic, J. C. C. Sim-Stark, S. F. Haydock, P. F. Leadlay, Chem. Biol. 2008, 15, 1231-1240.

[9] M. H. Medema, R. Kottmann, P. Yilmaz, M. Cummings, J. B. Biggins, K. Blin, I. de Bruijn, Y. H. Chooi, J. Claesen, R. C. Coates, P. Cruz-Morales, S. Duddela, S. Düsterhus, D. J. Edwards, D. P. Fewer, N. Garg, C. Geiger, J. P. Gomez-Escribano, A. Greule, M. Hadjithomas, A. S. Haines, E. J. N. Helfrich, M. L. Hillwig, K. Ishida, A. C. Jones, C. S. Jones, K. Jungmann, C. Kegler, H. U. Kim, P. Kötter, D. Krug, J. Masschelein, A. V. Melnik, S. M. Mantovani, E. A. Monroe, M. Moore, N. Moss, H.-W. Nützmann, G. Pan, A. Pati, D. Petras, F. J. Reen, F. Rosconi, Z. Rui, Z. Tian, N. J. Tobias, Y. Tsunematsu, P. Wiemann, E. Wyckoff, X. Yan, G. Yim, F. Yu, Y. Xie, B. Aigle, A. K. Apel, C. J. Balibar, E. P. Balskus, F. Barona-Gómez, A. Bechthold, H. B. Bode, R. Borriss, S. F. Brady, A. A. Brakhage, P. Caffrey, Y.-Q. Cheng, J. Clardy, R. J. Cox, R. De Mot, S. Donadio, M. S. Donia, W. A. van der Donk, P. C. Dorrestein, S. Doyle, A. J. M.

Driessen, M. Ehling-Schulz, K.-D. Entian, M. A. Fischbach, L. Gerwick, W. H. Gerwick, H. Gross, B. Gust, C. Hertweck, M. Höfte, S. E. Jensen, J. Ju, L. Katz, L. Kaysser, J. L. Klassen, N. P. Keller, J. Kormanec, O. P. Kuipers, T. Kuzuyama, N. C. Kyrpides, H.-J. Kwon, S. Lautru, R. Lavigne, C. Y. Lee, B. Linquan, X. Liu, W. Liu, et al., Nat. Chem. Biol. 2015, 11, 625-631.

[10] L. A. Kelley, S. Mezulis, C. M. Yates, M. N. Wass, M. J. E. Sternberg, Nat. Protoc. 2015, $10,845-858$. 


\section{Physicochemical and spectroscopic data}

\section{Thaxteramide A1}

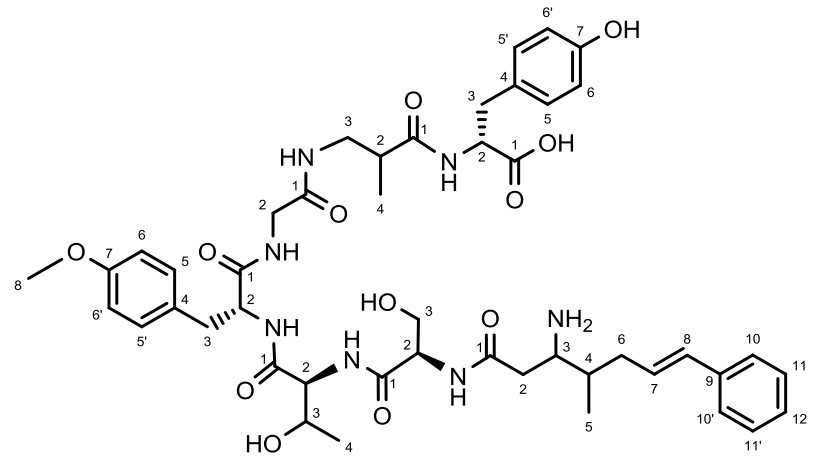

Thaxteramide A1 was isolated as described in procedure $\mathrm{B}$ and purified using systems $\mathrm{P}$ and SP1, successively. White solid, $1.5 \mathrm{mg},{ }^{1} \mathrm{H}$ NMR (700 MHz, $\left.\mathrm{CD}_{3} \mathrm{OD}\right)$ and ${ }^{13} \mathrm{C} \mathrm{NMR}$ as described in NMR table S1; HRMS (ESI+): $m / z$ calcd for $\mathrm{C}_{46} \mathrm{H}_{61} \mathrm{~N}_{7} \mathrm{O}_{12}+\mathrm{H}^{+}: 904.44510\left[M+\mathrm{H}^{+}\right]$, found: 904.44628, $\Delta \mathrm{m} 1.30 \mathrm{ppm} ;$ HPLC $t_{\mathrm{R}}=6.91$ (purity = 99\%; System A).

Table S1: NMR data of Thaxteramide $\mathrm{A} 1$ in $\mathrm{CD}_{3} \mathrm{OD}$

\begin{tabular}{|c|c|c|c|c|c|c|c|}
\hline $\begin{array}{c}\text { Partial } \\
\text { structure }\end{array}$ & $\mathbf{C}$ & Type & $\boldsymbol{\delta}_{\mathrm{C}}$ & $\delta_{H}($ mult., $J$ in $\mathrm{Hz})$ & НМBC & COSY & ROESY \\
\hline \multirow{8}{*}{ Tyrosine } & 1 & $\mathrm{CO}$ & 178.4 & - & - & - & \\
\hline & 2 & $\mathrm{CH}$ & 57.2 & $4.48(1 \mathrm{H}, \mathrm{dd}, 8.7,4.6)$ & $38.0,130.0,176.5,178.4$ & $3.12,2.83$ & $7.05,3.12,2.83$ \\
\hline & 3 & $\mathrm{CH}_{2}$ & 38.0 & $3.16-3.08(1 \mathrm{H}, \mathrm{m})^{*}$ & $57.2,130.0,131.2,178.4$ & $4.48,2.83$ & $2.83,7.05$ \\
\hline & & & & $2.83(1 \mathrm{H}, \mathrm{dd}, 14.1,9.0)$ & $57.2,130.0,131.2,178.4$ & $4.48,3.12$ & $3.12,7.05$ \\
\hline & 4 & $\mathrm{C}$ & 130.0 & - & - & - & \\
\hline & 5,5 & $\mathrm{CH}$ & 131.2 & $7.05(2 \mathrm{H}, \mathrm{d}, 8.4)$ & $115.8,156.7,38.0$ & 6.67 & 6.67 \\
\hline & 6,6 & $\mathrm{CH}$ & 115.8 & $6.67(2 \mathrm{H}, \mathrm{d}, 8.4)$ & $130.0,131.2,156.7$ & 7.05 & 7.05 \\
\hline & 7 & $\mathrm{C}$ & 156.7 & - & - & - & \\
\hline \multirow{5}{*}{$\begin{array}{l}\text { Methyl- } \beta \text { - } \\
\text { Alanine }\end{array}$} & 1 & $\mathrm{CO}$ & 176.5 & - & - & - & \\
\hline & 2 & $\mathrm{CH}$ & 41.3 & $2.59-2.52(1 \mathrm{H}, \mathrm{m})$ & $15.1,43.9,176.5$ & $3.12,0.96$ & $3.29,3.12,0.96$ \\
\hline & 3 & $\mathrm{CH}_{2}$ & 43.9 & $3.30-3.27(1 \mathrm{H}, \mathrm{m})^{\#}$ & $15.1,41.3,171.5,176.5$ & 3.12 & 3.12 \\
\hline & & & & $3.16-3.08(1 \mathrm{H}, \mathrm{m})^{*}$ & $15.1,41.3,171.5,176.5$ & $3.29,2.55$ & 3.29 \\
\hline & 4 & $\mathrm{CH}_{3}$ & 15.1 & $0.96(3 \mathrm{H}, \mathrm{d}, 6.9)$ & $41.3,43.9,176.5$ & 2.55 & 2.55 \\
\hline \multirow{3}{*}{ Glycine } & 1 & $\mathrm{CO}$ & 171.5 & - & - & - & \\
\hline & 2 & $\mathrm{CH}_{2}$ & 43.4 & $3.89(1 \mathrm{H}, \mathrm{d}, 16.3)^{*}$ & $171.5,173.9$ & 3.72 & \\
\hline & & & & $3.72(1 \mathrm{H}, \mathrm{d}, 16.5)$ & $171.5,173.9$ & 3.89 & \\
\hline
\end{tabular}

* Overlapped signals

\# Signal under solvent peak 
Table S1: NMR data of Thaxteramide $\mathrm{A} 1$ in $\mathrm{CD}_{3} \mathrm{OD}$ (continued)

\begin{tabular}{|c|c|c|c|c|c|c|c|}
\hline $\begin{array}{l}\text { Partial } \\
\text { structure }\end{array}$ & $\mathbf{C}$ & Type & $\boldsymbol{\delta}_{\mathbf{C}}$ & $\delta_{\mathrm{H}}($ mult., $J$ in $\mathrm{Hz})$ & НМВС & COSY & ROESY \\
\hline \multirow{9}{*}{$\begin{array}{l}\text { O- } \\
\text { Methyl- } \\
\text { Tyrosine }\end{array}$} & 1 & $\mathrm{CO}$ & 173.9 & - & - & - & - \\
\hline & 2 & $\mathrm{CH}$ & 56.6 & $4.56(1 \mathrm{H}, \mathrm{dd}, 9.7,5.2)$ & $37.4,130.2,172.7,173.9$ & $3.12,2.88$ & $7.15,2.88,3.12$ \\
\hline & 3 & $\mathrm{CH}_{2}$ & 37.4 & $3.16-3.08(1 \mathrm{H}, \mathrm{m})$ & $56.6,130.2,131.2,173.9$ & $4.56,2.88$ & $2.88,7.15$ \\
\hline & & & & $2.88(1 \mathrm{H}, \mathrm{dd}, 14.0,9.8)$ & $56.6,130.2,131.2,173.9$ & $4.56,3.12$ & $3.12,7.15$ \\
\hline & 4 & $\mathrm{C}$ & 130.2 & - & - & - & - \\
\hline & 5,5 & $\mathrm{CH}$ & 131.2 & $7.15(2 \mathrm{H}, \mathrm{d}, 8.5)$ & $114.7,159.8,37.4$ & 6.81 & 6.81 \\
\hline & 6,6 & $\mathrm{CH}$ & 114.7 & $6.81(2 \mathrm{H}, \mathrm{d}, 8.6)$ & $130.2,131.2,159.8$ & 7.15 & $7.15,3.75$ \\
\hline & 7 & $\mathrm{C}$ & 159.8 & - & - & - & - \\
\hline & 8 & $\mathrm{OCH}_{3}$ & 55.4 & $3.75(3 \mathrm{H}, \mathrm{s})$ & 159.8 & & 6.81 \\
\hline \multirow{4}{*}{ Threonine } & 1 & $\mathrm{CO}$ & 172.7 & - & - & - & - \\
\hline & 2 & $\mathrm{CH}$ & 60.3 & $4.22(1 \mathrm{H}, \mathrm{d}, 3.6)$ & $20.0,67.6,172.7$ & 4.19 & 1.06 \\
\hline & 3 & $\mathrm{CH}$ & 67.6 & 4.21-4.17 (1H, m) & 20.0 & $4.22,1.06$ & 1.06 \\
\hline & 4 & $\mathrm{CH}_{3}$ & 20.0 & $1.06(3 \mathrm{H}, \mathrm{d}, 5.3)$ & $60.3,67.6$ & 4.19 & 4.19 \\
\hline \multirow{4}{*}{ Serine } & 1 & $\mathrm{CO}$ & 173.0 & - & - & - & - \\
\hline & 2 & $\mathrm{CH}$ & 57.8 & $4.42(1 \mathrm{H}, \mathrm{t}, 5.3)$ & $62.3,173.0$ & $3.79,3.90$ & $3.79,3.90$ \\
\hline & 3 & $\mathrm{CH}_{2}$ & 62.3 & $3.90(1 \mathrm{H}, \mathrm{dd}, 11.0,5.4)^{*}$ & $57.8,173.0$ & $4.42,3.79$ & \\
\hline & & & & $3.79(1 \mathrm{H}, \mathrm{dd}, 11.0,5.4)$ & $57.8,173.0$ & $4.42,3.90$ & \\
\hline \multirow{14}{*}{$\begin{array}{l}\text { Lipid } \\
\text { chain }\end{array}$} & 1 & $\mathrm{CO}$ & 173.2 & - & - & - & - \\
\hline & 2 & $\mathrm{CH}_{2}$ & 33.8 & $2.78(1 \mathrm{H}, \mathrm{dd}, 16.4,3.4)$ & $36.7,53.2,173.2$ & $2.70,3.63$ & $1.05,2.78,2.70$ \\
\hline & & & & $2.70(1 \mathrm{H}, \mathrm{dd}, 16.5,9.7)$ & $36.7,53.2,173.2$ & $2.78,3.63$ & $3.63,1.05,2.78,2.70$ \\
\hline & 3 & $\mathrm{CH}$ & 53.2 & $3.63(1 \mathrm{H}, \mathrm{ddd}, 9.6,3.6,4.8)$ & $14.5,33.8,36.7,173.2$ & $2.09,2.78,2.70$ & $2.09,2.78,2.70$ \\
\hline & 4 & $\mathrm{CH}$ & 36.7 & $2.12-2.05(1 \mathrm{H}, \mathrm{m})$ & $14.5,33.8,37.4,53.2,127.8$ & $1.05,2.19,3.63$ & $3.63,1.05,6.22$ \\
\hline & 5 & $\mathrm{CH}_{3}$ & 14.5 & $1.05(3 \mathrm{H}, \mathrm{d}, 5.8)$ & $36.7,53.2$ & 2.09 & 2.09 \\
\hline & 6 & $\mathrm{CH}_{2}$ & 37.4 & $2.38(1 \mathrm{H}, \mathrm{dt}, 14.0,7.0)$ & $14.5,36.7,53.2,127.8,133.7$ & $6.22,2.19$ & $2.19,6.22,6.49$ \\
\hline & & & & $2.19(1 \mathrm{H}, \mathrm{dt}, 14.5,7.2)$ & $14.5,36.7,53.2,127.8,133.7$ & $6.22,2.38,2.09$ & $2.386 .22,6.49$ \\
\hline & 7 & $\mathrm{CH}$ & 127.8 & $6.22(1 \mathrm{H}, \mathrm{dt}, 15.8,7.2)$ & $138.4,37.4$ & $6.49,2.22,2.19$ & $7.36,2.38,2.19$ \\
\hline & 8 & $\mathrm{CH}$ & 133.7 & $6.49(1 \mathrm{H}, \mathrm{d}, 15.8)$ & $138.4,126.9,37.4$ & 6.22 & $7.36,2.38,2.19$ \\
\hline & 9 & $\mathrm{C}$ & 138.4 & - & - & - & - \\
\hline & $10,10^{\prime}$ & $\mathrm{CH}$ & 126.9 & $7.36(2 \mathrm{H}, \mathrm{d}, 7.5)$ & $128.0,134.0$ & 7.26 & $6.49,6.22$ \\
\hline & 11,11 & $\mathrm{CH}$ & 129.3 & $7.26(2 \mathrm{H}, \mathrm{t}, 7.6)$ & 138.8 & $7.36,7.18$ & \\
\hline & 12 & $\mathrm{CH}$ & 128.0 & $7.18(1 \mathrm{H}, \mathrm{t}, 7.4)$ & 126.9 & 7.26 & \\
\hline
\end{tabular}

* Overlapped signals 


\section{Thaxteramide A2}

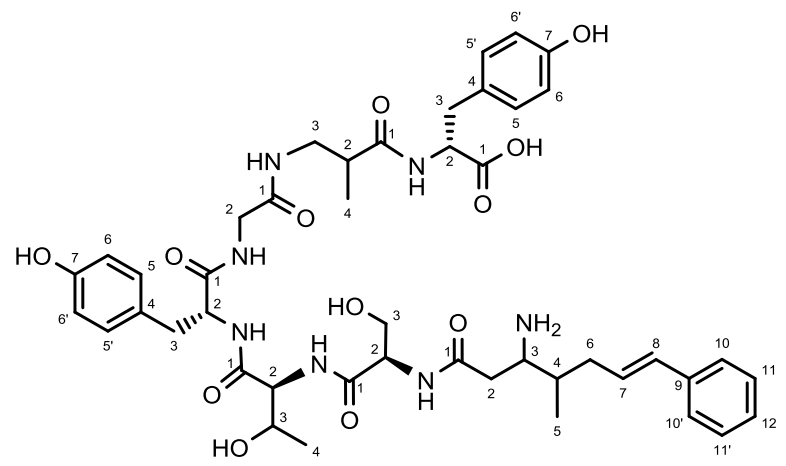

Thaxteramide A2 was isolated as described in procedure $\mathrm{B}$ and purified using systems $\mathrm{P}$ and SP1, successively. White solid, $0.1 \mathrm{mg},{ }^{1} \mathrm{H}$ NMR (700 MHz, $\mathrm{CD}_{3} \mathrm{OD}$ ) and ${ }^{13} \mathrm{C}$ NMR as described in NMR table S2; HRMS (ESI+): $m / z$ calcd for $\mathrm{C}_{45} \mathrm{H}_{59} \mathrm{~N}_{7} \mathrm{O}_{12}+\mathrm{H}^{+}: 890.42945\left[M+\mathrm{H}^{+}\right]$, found: 890.42856, $\Delta \mathrm{m}-1.00$ ppm; HPLC $t_{\mathrm{R}}=6.19$ (purity $=99 \%$; System A).

Table S2: NMR data of Thaxteramide A2 in $\mathrm{CD}_{3} \mathrm{OD}$

\begin{tabular}{|c|c|c|c|c|c|c|}
\hline $\begin{array}{c}\text { Partial } \\
\text { structure }\end{array}$ & $\mathbf{C}$ & Type & $\boldsymbol{\delta}_{\mathrm{C}}$ & $\delta_{\mathbf{H}}($ mult., $J$ in $\mathbf{H z})$ & HMBC & COSY \\
\hline \multirow{8}{*}{ Tyrosine } & 1 & $\mathrm{CO}$ & 178.6 & - & - & - \\
\hline & 2 & $\mathrm{CH}$ & 57.4 & $4.46(1 \mathrm{H}, \mathrm{dd}, 8.8,4.4)$ & $38.1,130.0,176.1,178.6$ & $3.14,2.82$ \\
\hline & 3 & $\mathrm{CH}_{2}$ & 38.1 & $3.14(1 \mathrm{H}, \mathrm{dd}, 14.0,4.3)$ & $57.4,130.0,131.1,178.6$ & $4.46,2.82$ \\
\hline & & & & $2.82(1 \mathrm{H}, \mathrm{dd}, 13.9,8.9)$ & $57.4,130.0,131.1,178.6$ & $4.46,3.14$ \\
\hline & 4 & $\mathrm{C}$ & 130.0 & - & - & - \\
\hline & 5,5 & $\mathrm{CH}$ & 131.1 & $7.05(2 \mathrm{H}, \mathrm{d}, 8.5)$ & $115.7,156.4,38.1$ & 6.66 \\
\hline & 6,6 & $\mathrm{CH}$ & 115.7 & $6.66(2 \mathrm{H}, \mathrm{d}, 8.5)$ & $130.0,156.4$ & 7.05 \\
\hline & 7 & $\mathrm{C}$ & 156.4 & - & - & - \\
\hline \multirow{5}{*}{$\begin{array}{l}\text { Methyl- } \beta \text { - } \\
\text { Alanine }\end{array}$} & 1 & $\mathrm{CO}$ & 176.1 & - & - & - \\
\hline & 2 & $\mathrm{CH}$ & 41.2 & $2.59-2.52(1 \mathrm{H}, \mathrm{m})$ & $15.0,43.8,176.1$ & $3.34,3.06,0.95$ \\
\hline & 3 & $\mathrm{CH}_{2}$ & 43.8 & $3.36-3.32(1 \mathrm{H}, \mathrm{m})^{\#}$ & $15.0,41.2,171.3,176.1$ & $3.06,2.55$ \\
\hline & & & & $3.06(1 \mathrm{H}, \mathrm{dd}, 13.2,10.1)$ & $15.0,41.2,171.3,176.1$ & $3.34,2.55$ \\
\hline & 4 & $\mathrm{CH}_{3}$ & 15.0 & $0.95(3 \mathrm{H}, \mathrm{d}, 6.9)$ & $41.2,43.8,176.1$ & 2.55 \\
\hline \multirow{3}{*}{ Glycine } & 1 & $\mathrm{CO}$ & 171.3 & - & - & - \\
\hline & 2 & $\mathrm{CH}_{2}$ & 43.2 & $3.89(1 \mathrm{H}, \mathrm{d}, 16.5)^{*}$ & $171.3,173.7$ & 3.72 \\
\hline & & & & $3.72(1 \mathrm{H}, \mathrm{d}, 16.6)$ & $171.3,173.7$ & 3.89 \\
\hline
\end{tabular}

* Overlapped signals

\# Signal under solvent peak 
Table S2: NMR data of Thaxteramide $\mathrm{A} 2$ in $\mathrm{CD}_{3} \mathrm{OD}$ (continued)

\begin{tabular}{|c|c|c|c|c|c|c|}
\hline $\begin{array}{c}\text { Partial } \\
\text { structure }\end{array}$ & $\mathbf{C}$ & Type & $\boldsymbol{\delta}_{\mathbf{C}}$ & $\delta_{H}($ mult., $J$ in $\mathrm{Hz})$ & HMBC & COSY \\
\hline \multirow{8}{*}{ Tyrosine } & 1 & $\mathrm{CO}$ & 173.7 & - & - & - \\
\hline & 2 & $\mathrm{CH}$ & 56.7 & $4.54(1 \mathrm{H}, \mathrm{dd}, 9.6,5.2)$ & $37.5,129.0,172.3,173.7$ & $3.08,2.85$ \\
\hline & 3 & $\mathrm{CH}_{2}$ & 37.5 & $3.08(1 \mathrm{H}, \mathrm{dd}, 14.3,5.6)$ & $56.7,129.0,131.1,173.7$ & $4.54,2.85$ \\
\hline & & & & $2.85(1 \mathrm{H}, \mathrm{dd}, 13.8,9.6)$ & $56.7,129.0,131.1,173.7$ & $4.54,3.08$ \\
\hline & 4 & $\mathrm{C}$ & 129.0 & - & - & - \\
\hline & 5,5 & $\mathrm{CH}$ & 131.1 & $7.06(2 \mathrm{H}, \mathrm{d}, 8.5)$ & $159.8,37.5$ & 6.68 \\
\hline & 6,6 & $\mathrm{CH}$ & 115.7 & $6.68(2 \mathrm{H}, \mathrm{d}, 8.5)$ & $129.0,157.2$ & 7.06 \\
\hline & 7 & $\mathrm{C}$ & 157.2 & - & - & - \\
\hline \multirow{4}{*}{ Threonine } & 1 & $\mathrm{CO}$ & 172.3 & - & - & - \\
\hline & 2 & $\mathrm{CH}$ & 60.2 & $4.22(1 \mathrm{H}, \mathrm{d}, 3.6)$ & $20.0,67.5,172.3$ & 4.19 \\
\hline & 3 & $\mathrm{CH}$ & 67.5 & $4.19(1 \mathrm{H}, \mathrm{dd}, 3.8,6.4)$ & & $4.22,1.07$ \\
\hline & 4 & $\mathrm{CH}_{3}$ & 20.0 & $1.07(3 \mathrm{H}, \mathrm{d}, 6.4)$ & $60.2,67.5$ & 4.19 \\
\hline \multirow{4}{*}{ Serine } & 1 & $\mathrm{CO}$ & 172.7 & - & - & - \\
\hline & 2 & $\mathrm{CH}$ & 57.7 & $4.41(1 \mathrm{H}, \mathrm{t}, 5.3)$ & $62.2,172.7$ & $3.79,3.89$ \\
\hline & 3 & $\mathrm{CH}_{2}$ & 62.2 & $3.92-3.87(1 \mathrm{H}, \mathrm{m})^{*}$ & 57.7 & $4.41,3.79$ \\
\hline & & & & $3.79(1 \mathrm{H}, \mathrm{dd}, 11.0,5.1)$ & $57.7,172.7$ & $4.41,3.89$ \\
\hline \multirow{14}{*}{$\begin{array}{l}\text { Lipid } \\
\text { chain }\end{array}$} & 1 & $\mathrm{CO}$ & ND & - & - & - \\
\hline & 2 & $\mathrm{CH}_{2}$ & ND & $2.78-2.72(1 \mathrm{H}, \mathrm{m})$ & & $2.66,3.59$ \\
\hline & & & & $2.71-2.64(1 \mathrm{H}, \mathrm{m})$ & & $2.75,3.59$ \\
\hline & 3 & $\mathrm{CH}$ & 53.1 & $3.62-3.56(1 \mathrm{H}, \mathrm{m})$ & & $2.75,2.66$ \\
\hline & 4 & $\mathrm{CH}$ & 37.0 & $2.10-2.05(1 \mathrm{H}, \mathrm{m})$ & & $1.05,2.19,2.37$ \\
\hline & 5 & $\mathrm{CH}_{3}$ & 14.4 & $1.05(3 \mathrm{H}, \mathrm{d}, 6.8)$ & $37.0,37.5,53.1$ & 2.07 \\
\hline & 6 & $\mathrm{CH}_{2}$ & 37.5 & $2.37(1 \mathrm{H}, \mathrm{dt}, 13.2,6.6)$ & $14.4,37.0,53.0,128.0,133.6$ & $6.23,2.17$ \\
\hline & & & & $2.19-2.14(1 \mathrm{H}, \mathrm{m})$ & $14.4,37.0,53.0,128.0,133.6$ & $6.23,2.37,2.07$ \\
\hline & 7 & $\mathrm{CH}$ & 128.0 & $6.23(1 \mathrm{H}, \mathrm{dt}, 15.8,7.3)$ & 138.3 & $6.48,2.36,2.19$ \\
\hline & 8 & $\mathrm{CH}$ & 133.6 & $6.48(1 \mathrm{H}, \mathrm{d}, 15.6)$ & $138.3,126.9,37.3$ & 6.23 \\
\hline & 9 & $\mathrm{C}$ & 138.3 & - & - & - \\
\hline & $10,10^{\prime}$ & $\mathrm{CH}$ & 126.9 & $7.36(2 \mathrm{H}, \mathrm{d}, 7.3)$ & $128.0,133.6$ & 7.26 \\
\hline & 11,11 , & $\mathrm{CH}$ & 129.2 & $7.26(2 \mathrm{H}, \mathrm{t}, 7.7)$ & 138.3 & $7.36,7.18$ \\
\hline & 12 & $\mathrm{CH}$ & 128.0 & $7.18(1 \mathrm{H}, \mathrm{t}, 7.3)$ & 126.9 & 7.26 \\
\hline
\end{tabular}

* Overlapped signals

ND: not determined 


\section{Thaxteramide B1}

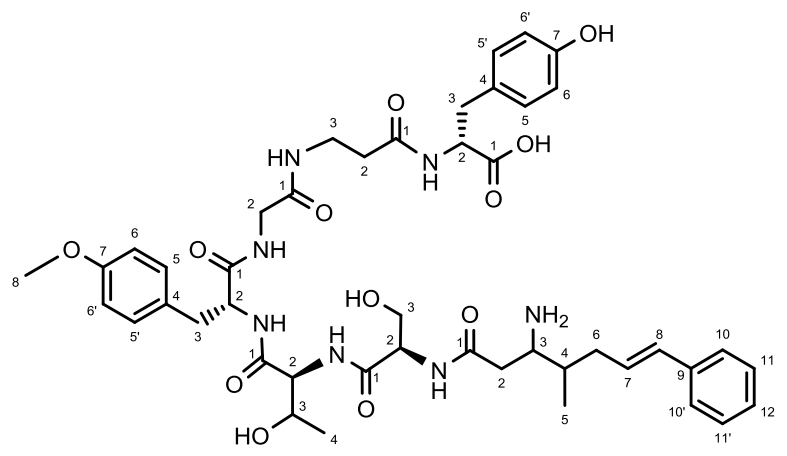

Thaxteramide $\mathrm{B} 1$ was isolated as described in procedure $\mathrm{B}$ and purified using systems $\mathrm{P}$ and SP1, successively. White solid, $0.6 \mathrm{mg},{ }^{1} \mathrm{H}$ NMR $\left(700 \mathrm{MHz}, \mathrm{CD}_{3} \mathrm{OD}\right)$ and ${ }^{13} \mathrm{C}$ NMR as described in NMR table S3; HRMS (ESI+): $m / z$ calcd for $\mathrm{C}_{45} \mathrm{H}_{59} \mathrm{~N}_{7} \mathrm{O}_{12}+\mathrm{H}^{+}: 890.42945\left[M+\mathrm{H}^{+}\right]$, found: 890.42919, $\Delta \mathrm{m}-0.29$ ppm; HPLC $t_{\mathrm{R}}=6.70$ (purity $=99 \%$; System A).

Table S3: NMR data of Thaxteramide $\mathrm{B} 1$ in $\mathrm{CD}_{3} \mathrm{OD}$

\begin{tabular}{|c|c|c|c|c|c|c|}
\hline $\begin{array}{c}\text { Partial } \\
\text { structure }\end{array}$ & $\mathbf{C}$ & Type & $\boldsymbol{\delta}_{\mathrm{C}}$ & $\delta_{\mathbf{H}}($ mult., $J$ in $\mathbf{H z})$ & HМBC & COSY \\
\hline \multirow{8}{*}{ Tyrosine } & 1 & $\mathrm{CO}$ & 178.5 & - & - & - \\
\hline & 2 & $\mathrm{CH}$ & 57.7 & 4.44-4.39 $(1 \mathrm{H}, \mathrm{m}) *$ & $37.9,130.1,178.5$ & $3.13,2.84$ \\
\hline & 3 & $\mathrm{CH}_{2}$ & 37.9 & $3.13(1 \mathrm{H}, \mathrm{dd}, 13.7,8.5)$ & $57.7,130.1,131.0,178.5$ & $4.41,2.84$ \\
\hline & & & & $2.84(1 \mathrm{H}, \mathrm{dd}, 14.2,8.9)$ & $57.7,130.1,131.0,178.5$ & $4.41,3.13$ \\
\hline & 4 & $\mathrm{C}$ & 130.1 & - & - & - \\
\hline & 5,5 & $\mathrm{CH}$ & 131.0 & $7.05(2 \mathrm{H}, \mathrm{d}, 8.3)$ & $37.9,156.5$ & 6.67 \\
\hline & 6,6 & $\mathrm{CH}$ & 115.6 & $6.67(2 \mathrm{H}, \mathrm{d}, 8.3)$ & $130.1,156.5$ & 7.05 \\
\hline & 7 & $\mathrm{C}$ & 156.5 & - & - & - \\
\hline \multirow{5}{*}{$\beta$-Alanine } & 1 & $\mathrm{CO}$ & 172.6 & - & - & - \\
\hline & 2 & $\mathrm{CH}_{2}$ & 36.2 & $2.45-2.38(1 \mathrm{H}, \mathrm{m})^{*}$ & $36.9,172.6$ & $2.29,3.48,3.34$ \\
\hline & & & & $2.29(1 \mathrm{H}, \mathrm{dt}, 14.1,5.8)$ & $36.9,172.6$ & $2.42,3.48,3.34$ \\
\hline & 3 & $\mathrm{CH}_{2}$ & 36.9 & $3.48(1 \mathrm{H}, \mathrm{dt}, 12.6,6.3)$ & $36.2,172.6$ & $2.29,2.42,3.34$ \\
\hline & & & & $3.36-3.32(1 \mathrm{H}, \mathrm{m})^{\#}$ & $36.2,172.6$ & $2.29,2.42,3.48$ \\
\hline \multirow{3}{*}{ Glycine } & 1 & $\mathrm{CO}$ & 170.4 & - & - & - \\
\hline & 2 & $\mathrm{CH}_{2}$ & 43.4 & $3.91(1 \mathrm{H}, \mathrm{d}, 16.7)^{*}$ & $170.4,173.7$ & 3.66 \\
\hline & & & & $3.66(1 \mathrm{H}, \mathrm{d}, 16.6)$ & $170.4,173.7$ & 3.91 \\
\hline \multirow{9}{*}{$\begin{array}{l}\text { O-Methyl- } \\
\text { Tyrosine }\end{array}$} & 1 & $\mathrm{CO}$ & 173.1 & - & - & - \\
\hline & 2 & $\mathrm{CH}$ & 56.5 & $4.53(1 \mathrm{H}, \mathrm{dd}, 9.7,5.2)$ & $37.1,130.2,173.1$ & $3.13,2.88$ \\
\hline & 3 & $\mathrm{CH}_{2}$ & 37.1 & $3.13(1 \mathrm{H}, \mathrm{dd}, 13.5,7.8)$ & $56.5,130.1,173.1$ & 4.532 .88 \\
\hline & & & & $2.88(1 \mathrm{H}, \mathrm{dd}, 14.0,9.9)$ & $56.5,130.1,173.1$ & $4.53,3.13$ \\
\hline & 4 & $\mathrm{C}$ & 130.1 & - & - & - \\
\hline & 5,5 & $\mathrm{CH}$ & 131.0 & $7.15(2 \mathrm{H}, \mathrm{d}, 8.5)$ & $159.7,37.1$ & 6.82 \\
\hline & $6,6^{\prime}$ & $\mathrm{CH}$ & 114.5 & $6.82(2 \mathrm{H}, \mathrm{d}, 8.6)$ & $130.1,159.7$ & 7.15 \\
\hline & 7 & $\mathrm{C}$ & 159.7 & - & - & - \\
\hline & 8 & $\mathrm{OCH}_{3}$ & 55.2 & $3.75(3 \mathrm{H}, \mathrm{s})$ & 159.7 & \\
\hline
\end{tabular}

* Overlapped signals 
Table S3: NMR data of thaxteramide $\mathrm{B} 1$ in $\mathrm{CD}_{3} \mathrm{OD}$ (continued)

\begin{tabular}{|c|c|c|c|c|c|c|}
\hline $\begin{array}{c}\text { Partial } \\
\text { structure }\end{array}$ & $\mathbf{C}$ & Type & $\boldsymbol{\delta}_{\mathrm{C}}$ & $\delta_{H}$ (mult., $J$ in $\mathrm{Hz}$ ) & НМВС & COSY \\
\hline \multirow{4}{*}{ Threonine } & 1 & $\mathrm{CO}$ & 172.2 & - & - & - \\
\hline & 2 & $\mathrm{CH}$ & 60.2 & $4.20(1 \mathrm{H}, \mathrm{d}, 3.9)$ & 172.2 & 4.16 \\
\hline & 3 & $\mathrm{CH}$ & 67.7 & $4.16(1 \mathrm{H}, \mathrm{dq}, 4.2,6.0)$ & & $4.21,1.05$ \\
\hline & 4 & $\mathrm{CH}_{3}$ & 20.0 & $1.04(3 \mathrm{H}, \mathrm{d}, 6.6)$ & $60.2,67.7$ & 4.16 \\
\hline \multirow{4}{*}{ Serine } & 1 & $\mathrm{CO}$ & 173.0 & - & - & - \\
\hline & 2 & $\mathrm{CH}$ & 57.7 & 4.44-4.39 $(1 \mathrm{H}, \mathrm{m})^{*}$ & $62.2,173.0$ & $3.81,3.90$ \\
\hline & 3 & $\mathrm{CH}_{2}$ & 62.2 & $3.90(1 \mathrm{H}, \mathrm{dd}, 10.5,5.9)^{*}$ & & $4.42,3.81$ \\
\hline & & & & $3.81(1 \mathrm{H}, \mathrm{dd}, 10.9,4.9)$ & & $4.42,3.90$ \\
\hline \multirow{14}{*}{ Lipid chain } & 1 & $\mathrm{CO}$ & 173.1 & - & - & - \\
\hline & 2 & $\mathrm{CH}_{2}$ & 33.9 & $2.80-2.74(1 \mathrm{H}, \mathrm{m})$ & - & 2.69 \\
\hline & & & & $2.69(1 \mathrm{H}, \mathrm{dd}, 16.3,9.5)$ & $53.2,173.1$ & $2.77,3.62$ \\
\hline & 3 & $\mathrm{CH}$ & 53.2 & $3.64-3.59(1 \mathrm{H}, \mathrm{m})$ & - & $2.69,2.77,2.08$ \\
\hline & 4 & $\mathrm{CH}$ & 36.6 & $2.11-2.05(1 \mathrm{H}, \mathrm{m})$ & $14.4,33.9$ & $1.05,2.39,2.18,3$ \\
\hline & 5 & $\mathrm{CH}_{3}$ & 14.4 & $1.05(3 \mathrm{H}, \mathrm{d}, 7.0)$ & $37.2,53.2$ & 2.08 \\
\hline & 6 & $\mathrm{CH}_{2}$ & 37.2 & $2.43-2.34(1 \mathrm{H}, \mathrm{m})$ & $14.4,36.6,53.2,127.8,133.7$ & $2.18,2.08,6.23$ \\
\hline & & & & $2.14-2.22(1 \mathrm{H}, \mathrm{m})$ & $14.4,36.6,53.2,127.8,133.7$ & $2.39,2.08,6.23$ \\
\hline & 7 & $\mathrm{CH}$ & 127.8 & $6.23(1 \mathrm{H}, \mathrm{dt}, 14.9,7.5)$ & 138.4 & $6.49,2.18,2.39$ \\
\hline & 8 & $\mathrm{CH}$ & 133.7 & $6.49(1 \mathrm{H}, \mathrm{d}, 15.9)$ & $126.9,37.2$ & 6.23 \\
\hline & 9 & $\mathrm{C}$ & 138.4 & - & - & - \\
\hline & $10,10^{\prime}$ & $\mathrm{CH}$ & 126.9 & $7.36(2 \mathrm{H}, \mathrm{d}, 7.5)$ & $128.0,133.7$ & 7.26 \\
\hline & $11,11^{\prime}$ & $\mathrm{CH}$ & 129.3 & $7.26(2 \mathrm{H}, \mathrm{t}, 7.6)$ & 138.4 & $7.36,7.18$ \\
\hline & 12 & $\mathrm{CH}$ & 128.0 & $7.18(1 \mathrm{H}, \mathrm{t}, 7.3)$ & 126.9 & 7.26 \\
\hline
\end{tabular}

* Overlapped signals 


\section{Thaxteramide B2}

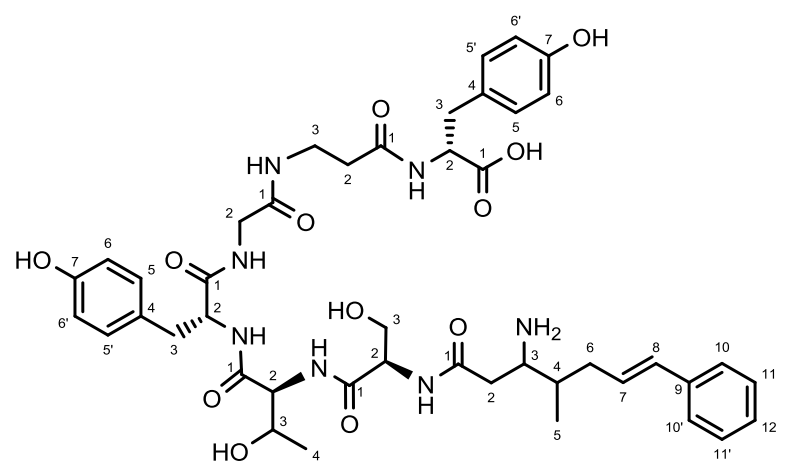

Thaxteramide B2 was isolated as described in procedure $\mathrm{B}$ and purified using systems $\mathrm{P}$ and $\mathrm{SP} 2$, successively. White solid, $0.06 \mathrm{mg},{ }^{1} \mathrm{H} \mathrm{NMR}\left(700 \mathrm{MHz}, \mathrm{CD}_{3} \mathrm{OD}\right)$ and ${ }^{13} \mathrm{C} \mathrm{NMR}$ as described in NMR table S4; HRMS (ESI+): $m / z$ calcd for $\mathrm{C}_{44} \mathrm{H}_{57} \mathrm{~N}_{7} \mathrm{O}_{12}+\mathrm{H}^{+}: 876.41462\left[M+\mathrm{H}^{+}\right]$, found: 876.41290, $\Delta \mathrm{m} 1.96$ ppm; HPLC $t_{\mathrm{R}}=5.93$ (purity $=95 \%$; System A).

Table S4: NMR data of thaxteramide $\mathrm{B} 2$ in $\mathrm{CD}_{3} \mathrm{OD}$

\begin{tabular}{|c|c|c|c|c|c|c|}
\hline $\begin{array}{c}\text { Partial } \\
\text { structure }\end{array}$ & $\mathbf{C}$ & Type & $\boldsymbol{\delta}_{\mathbf{C}}$ & $\delta_{H}$ (mult., $J$ in $\mathrm{Hz}$ ) & HМBC & COSY \\
\hline \multirow{8}{*}{ Tyrosine } & 1 & $\mathrm{CO}$ & 178.4 & - & - & - \\
\hline & 2 & $\mathrm{CH}$ & 57.7 & $4.43-4.39(1 \mathrm{H}, \mathrm{m})^{*}$ & $37.8,130.9,178.2$ & $3.12,2.85$ \\
\hline & 3 & $\mathrm{CH}_{2}$ & 37.4 & $3.12(1 \mathrm{H}, \mathrm{dd}, 13.7,4.5)^{*}$ & $57.7,130.9,178.2$ & 2.85 \\
\hline & & & & $2.85(1 \mathrm{H}, \mathrm{dd}, 14.3,6.2)^{*}$ & $57.7,130.9,178.2$ & $4.41,3.12$ \\
\hline & 4 & $\mathrm{C}$ & 130.9 & - & - & - \\
\hline & 5,5 & $\mathrm{CH}$ & 131.0 & $7.05(2 \mathrm{H}, \mathrm{d}, 8.3)$ & $156.4,37.4$ & 6.67 \\
\hline & 6,6 & $\mathrm{CH}$ & 115.8 & $6.67(2 \mathrm{H}, \mathrm{d}, 8.5)$ & $130.9,156.4$ & 7.05 \\
\hline & 7 & $\mathrm{C}$ & 156.4 & - & - & - \\
\hline \multirow{5}{*}{$\beta$-Alanine } & 1 & $\mathrm{CO}$ & 173.1 & - & - & - \\
\hline & 2 & $\mathrm{CH}_{2}$ & 36.2 & $2.44-2.35(1 \mathrm{H}, \mathrm{m})^{*}$ & $37.0,173.1$ & $2.29,3.47,3.35$ \\
\hline & & & & $2.29(1 \mathrm{H}, \mathrm{dt}, 12.9,6.4)$ & $37.0,173.1$ & $2.39,3.47,3.35$ \\
\hline & 3 & $\mathrm{CH}_{2}$ & 37.0 & $3.50-3.44(1 \mathrm{H}, \mathrm{m})$ & $36.2,173.1,171.9$ & $2.29,2.39,3.35$ \\
\hline & & & & $3.40-3.30(1 \mathrm{H}, \mathrm{m})^{\#}$ & $36.2,173.1,171.9$ & $2.29,2.39,3.47$ \\
\hline \multirow{3}{*}{ Glycine } & 1 & $\mathrm{CO}$ & 171.9 & - & - & - \\
\hline & 2 & $\mathrm{CH}_{2}$ & 43.1 & $3.89(1 \mathrm{H}, \mathrm{d}, 16.7)^{*}$ & $171.9,173.8$ & 3.66 \\
\hline & & & & $3.66(1 \mathrm{H}, \mathrm{d}, 16.8)$ & $171.9,173.8$ & 3.89 \\
\hline \multirow{8}{*}{ Tyrosine } & 1 & $\mathrm{CO}$ & 173.8 & - & - & - \\
\hline & 2 & $\mathrm{CH}$ & 56.7 & $4.51(1 \mathrm{H}, \mathrm{dd}, 9.5,5.5)$ & $37.4,128.5,172.4,173.8$ & $3.10,2.84$ \\
\hline & 3 & $\mathrm{CH}_{2}$ & 37.4 & $3.10(1 \mathrm{H}, \mathrm{dd}, 13.8,5.2)^{*}$ & $56.7,128.5,173.8$ & 2.84 \\
\hline & & & & $2.84(1 \mathrm{H}, \mathrm{dd}, 14.1,5.9)^{*}$ & $56.7,128.5,173.8$ & $4.51,3.10$ \\
\hline & 4 & $\mathrm{C}$ & 128.5 & - & - & - \\
\hline & 5,5 & $\mathrm{CH}$ & 131.0 & $7.06(2 \mathrm{H}, \mathrm{d}, 8.4)$ & $157.1,37.4$ & 6.69 \\
\hline & 6,6 & $\mathrm{CH}$ & 115.8 & $6.69(2 \mathrm{H}, \mathrm{d}, 8.5)$ & $128.5,157.1$ & 7.06 \\
\hline & 7 & $\mathrm{C}$ & 157.1 & - & - & - \\
\hline
\end{tabular}


Table S4: NMR data of thaxteramide $\mathrm{B} 2$ in $\mathrm{CD}_{3} \mathrm{OD}$ (continued)

\begin{tabular}{|c|c|c|c|c|c|c|}
\hline $\begin{array}{c}\text { Partial } \\
\text { structure }\end{array}$ & $\mathbf{C}$ & Type & $\boldsymbol{\delta}_{\mathrm{C}}$ & $\delta_{H}$ (mult., $J$ in $\mathbf{H z}$ ) & HМBC & COSY \\
\hline \multirow{4}{*}{ Threonine } & 1 & $\mathrm{CO}$ & 172.4 & - & - & - \\
\hline & 2 & $\mathrm{CH}$ & 60.2 & $4.22(1 \mathrm{H}, \mathrm{d}, 4.0)$ & $20.0,67.5,172.4$ & 4.15 \\
\hline & 3 & $\mathrm{CH}$ & 67.5 & $4.17-4.13(1 \mathrm{H}, \mathrm{m})$ & & $4.22,1.06$ \\
\hline & 4 & $\mathrm{CH}_{3}$ & 20.0 & $1.05(3 \mathrm{H}, \mathrm{d}, 4.3)$ & $60.2,67.5$ & 4.15 \\
\hline \multirow{4}{*}{ Serine } & 1 & $\mathrm{CO}$ & 172.8 & - & - & - \\
\hline & 2 & $\mathrm{CH}$ & 57.7 & 4.43-4.39 $(1 \mathrm{H}, \mathrm{m})^{*}$ & $62.2,172.8$ & $3.89,3.80$ \\
\hline & 3 & $\mathrm{CH}_{2}$ & 62.2 & $3.89(1 \mathrm{H}, \mathrm{dd}, 11.1,5.7)^{*}$ & $57.7,172.8$ & $4.41,3.80$ \\
\hline & & & & $3.80(1 \mathrm{H}, \mathrm{dd}, 11.0,5.1)$ & $57.7,172.8$ & $4.41,3.89$ \\
\hline \multirow{14}{*}{ Lipid chain } & 1 & $\mathrm{CO}$ & 173.1 & - & - & - \\
\hline & 2 & $\mathrm{CH}_{2}$ & 33.9 & $2.79-2.74(1 \mathrm{H}, \mathrm{m})$ & 173.1 & $2.68,3.63$ \\
\hline & & & & $2.68(1 \mathrm{H}, \mathrm{dd}, 16.0,9.3)$ & $53.0,173.1$ & $2.77,3.63$ \\
\hline & 3 & $\mathrm{CH}$ & 53.0 & $3.67-3.59(1 \mathrm{H}, \mathrm{m})$ & & $2.77,2.68$ \\
\hline & 4 & $\mathrm{CH}$ & 36.7 & $2.10-2.04(1 \mathrm{H}, \mathrm{m})$ & 53.0 & 1.06 \\
\hline & 5 & $\mathrm{CH}_{3}$ & 14.5 & $1.06(3 \mathrm{H}, \mathrm{d}, 4.7)$ & $36.7,37.1,53.0$ & 2.07 \\
\hline & 6 & $\mathrm{CH}_{2}$ & 37.1 & $2.41-2.35(1 \mathrm{H}, \mathrm{m})^{*}$ & $14.5,36.7,53.0,127.9,133.6$ & $6.23,2.18$ \\
\hline & & & & $2.21-2.16(1 \mathrm{H}, \mathrm{m})$ & $14.5,36.7,53.0,127.9,133.6$ & $6.23,2.38$ \\
\hline & 7 & $\mathrm{CH}$ & 127.9 & $6.23(1 \mathrm{H}, \mathrm{dt}, 15.1,7.6)$ & 138.4 & 6.49 \\
\hline & 8 & $\mathrm{CH}$ & 133.6 & $6.49(1 \mathrm{H}, \mathrm{d}, 15.9)$ & $138.4,126.9,37.1$ & 6.23 \\
\hline & 9 & $\mathrm{C}$ & 138.4 & - & - & - \\
\hline & 10,10 & $\mathrm{CH}$ & 126.9 & $7.36(2 \mathrm{H}, \mathrm{d}, 7.3)$ & $127.9,133.6$ & 7.26 \\
\hline & $11,11^{\prime}$ & $\mathrm{CH}$ & 129.3 & $7.26(2 \mathrm{H}, \mathrm{t}, 7.9)$ & 138.4 & $7.36,7.18$ \\
\hline & 12 & $\mathrm{CH}$ & 127.9 & $7.18(1 \mathrm{H}, \mathrm{t}, 7.3)$ & 126.9 & 7.26 \\
\hline
\end{tabular}

* Overlapped signals 


\section{Thaxteramide C}

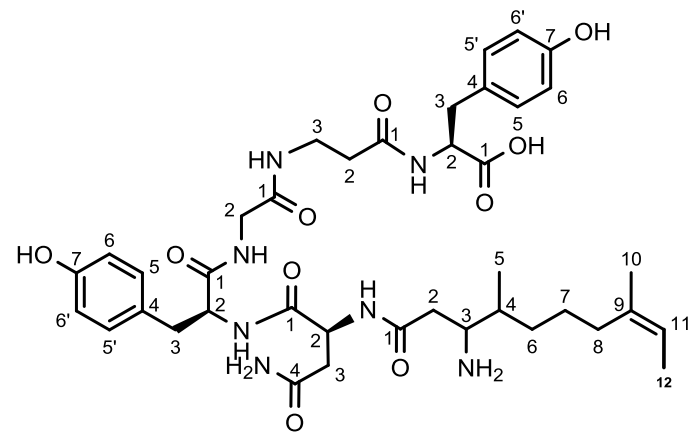

Thaxteramide $\mathrm{C}$ was isolated as described in procedure $\mathrm{B}$ and purified using systems $\mathrm{P}$ and SP2, successively. White solid, $0.45 \mathrm{mg},{ }^{1} \mathrm{H} \mathrm{NMR}\left(700 \mathrm{MHz}, \mathrm{CD}_{3} \mathrm{OD}\right)$ and ${ }^{13} \mathrm{C} \mathrm{NMR}$ as described in NMR table S5; HRMS (ESI+): $m / z$ calcd for $\mathrm{C}_{39} \mathrm{H}_{55} \mathrm{~N}_{7} \mathrm{O}_{10}+\mathrm{H}^{+}: 782.40832\left[M+\mathrm{H}^{+}\right]$, found: 782.40859, $\Delta \mathrm{m} 0.35$ ppm; HPLC $t_{\mathrm{R}}=5.96$ (purity = 95\%; System A).

Table S5: NMR data of thaxteramide $\mathrm{C}$ in $\mathrm{CD}_{3} \mathrm{OD}$

\begin{tabular}{|c|c|c|c|c|c|c|c|}
\hline $\begin{array}{l}\text { Partial } \\
\text { structure }\end{array}$ & C & Type & $\boldsymbol{\delta}_{\mathrm{C}}$ & $\delta_{\mathrm{H}}($ mult., $J$ in $\mathbf{H z})$ & HMBC & COSY & NOESY \\
\hline & 1 & $\mathrm{CO}$ & 178.2 & - & - & - & - \\
\hline \multirow[t]{8}{*}{ Tyrosine } & 2 & $\mathrm{CH}$ & 57.6 & $4.43(1 \mathrm{H}, \mathrm{dd}, 8.7,4.5)$ & $37.9,130.2,173.2,178.2$ & $3.14,2.83$ & $3.14,2.83$ \\
\hline & 3 & $\mathrm{CH}_{2}$ & 37.9 & $3.14(1 \mathrm{H}, \mathrm{dd}, 14.0,4.3)$ & $57.6,130.2,131.0178 .2$ & $4.43,2.83$ & $4.43,2.83$ \\
\hline & & & & $2.83(1 \mathrm{H}, \mathrm{dd}, 14.1,8.9)$ & $57.6,130.2,131.0178 .2$ & $4.43,3.14$ & $4.43,3.14$ \\
\hline & 4 & $\mathrm{C}$ & 130.2 & - & - & - & - \\
\hline & 5,5 & $\mathrm{CH}$ & 131.0 & $7.06(2 \mathrm{H}, \mathrm{d}, 8.4)$ & $116.0,37.9,156.7$ & 7.06 & 7.06 \\
\hline & 6,6 & $\mathrm{CH}$ & 116.0 & $6.67(2 \mathrm{H}, \mathrm{d}, 8.4)$ & $130.2,156.7$ & 6.67 & 6.67 \\
\hline & 7 & $\mathrm{C}$ & 156.7 & - & - & - & - \\
\hline & 1 & $\mathrm{CO}$ & 173.2 & - & - & - & - \\
\hline \multirow[t]{5}{*}{$\beta$-Alanine } & 2 & $\mathrm{CH}_{2}$ & 36.3 & $2.41(1 \mathrm{H}, \mathrm{ddd}, 14.4,5.6,8.5)$ & $36.9,173.2$ & $3.53,3.34,2.31$ & 3.53 \\
\hline & & & & $2.31(1 \mathrm{H}, \mathrm{ddd}, 14.4,5.8,5.8)$ & $36.9,173.2$ & $3.53,3.34,2.41$ & 3.53 \\
\hline & 3 & $\mathrm{CH}_{2}$ & 36.9 & $3.53(1 \mathrm{H}, \mathrm{dt}, 13.4,6.0)$ & $36.3,171.5,173.2$ & $3.34,2.41,2.31$ & $2.41,2.31$ \\
\hline & & & & $3.37-3.31(1 \mathrm{H})^{\#}$ & $36.3,171.5,173.2$ & & $2.41,2.31$ \\
\hline & 1 & $\mathrm{CO}$ & 171.5 & - & - & - & - \\
\hline \multirow[t]{3}{*}{ Glycine } & 2 & $\mathrm{CH}_{2}$ & 43.4 & $3.93(1 \mathrm{H}, \mathrm{d}, 16.9)$ & $171.5,174.1$ & 3.62 & 3.62 \\
\hline & & & & $3.62(1 \mathrm{H}, \mathrm{d}, 16.9)$ & $171.5,174.1$ & 3.93 & 3.93 \\
\hline & 1 & $\mathrm{CO}$ & 174.1 & - & - & - & - \\
\hline \multirow[t]{7}{*}{ Tyrosine } & 2 & $\mathrm{CH}$ & 57.1 & $4.40(1 \mathrm{H}, \mathrm{dd}, 9.1,5.8)$ & $36.7,128.8,173.4,174.1$ & $3.11,2.87$ & $3.11,2.87$ \\
\hline & 3 & $\mathrm{CH}_{2}$ & 36.7 & $3.11(1 \mathrm{H}, \mathrm{dd}, 14.0,5.6)$ & $57.1,128.8,131.0,174.1$ & $4.40,2.87$ & $4.40,2.87$ \\
\hline & & & & $2.87(1 \mathrm{H}, \mathrm{dd}, 14.0,9.2)$ & $57.1,128.8,131.0,174.1$ & $4.40,3.11$ & $4.40,3.11$ \\
\hline & 4 & $\mathrm{C}$ & 128.8 & - & - & - & - \\
\hline & 5,5 & $\mathrm{CH}$ & 131.0 & $7.06(2 \mathrm{H}, \mathrm{d}, 8.4)$ & $157.1,36.7$ & 7.06 & 7.06 \\
\hline & 6,6 & $\mathrm{CH}$ & 116.0 & $6.71(2 \mathrm{H}, \mathrm{d}, 8.4)$ & $128.8,157.1$ & 6.71 & 6.71 \\
\hline & 7 & $\mathrm{C}$ & 157.1 & - & - & - & - \\
\hline
\end{tabular}

\footnotetext{
${ }^{\#}$ Signal under solvent peak
} 
Table S5: NMR data of Thaxteramide $\mathrm{C}$ in $\mathrm{CD}_{3} \mathrm{OD}$ (continued)

\begin{tabular}{|c|c|c|c|c|c|c|c|}
\hline $\begin{array}{c}\text { Partial } \\
\text { structure }\end{array}$ & $\mathbf{C}$ & Type & $\boldsymbol{\delta}_{\mathrm{C}}$ & $\delta_{\mathbf{H}}($ mult., $J$ in $\mathrm{Hz})$ & HMBC & COSY & NOESY \\
\hline \multirow{5}{*}{ Asparagine } & 1 & $\mathrm{CO}$ & 173.4 & - & - & - & - \\
\hline & 2 & $\mathrm{CH}$ & 51.4 & $4.68(1 \mathrm{H}, \mathrm{dd}, 8.2,5.4)$ & $37.5,172.5,173.4,174.6$ & $2.56,2.67$ & $2.56,2.67$ \\
\hline & 3 & $\mathrm{CH}_{2}$ & 37.5 & $2.67(1 \mathrm{H}, \mathrm{dd}, 15.6,5.2)$ & $51.4,173.4,174.6$ & 2.56 & 4.68 \\
\hline & & & & $2.56(1 \mathrm{H}, \mathrm{dd}, 15.7,8.3)$ & & 2.67 & 4.68 \\
\hline & 4 & $\mathrm{CO}$ & 174.6 & - & - & - & - \\
\hline \multirow{15}{*}{$\begin{array}{l}\text { Lipid } \\
\text { chain }\end{array}$} & 1 & $\mathrm{CO}$ & 172.5 & - & - & - & - \\
\hline & 2 & $\mathrm{CH}_{2}$ & 35.5 & $2.65(1 \mathrm{H}, \mathrm{dd}, 16.1,4.0)$ & $36.3,54.3,172.5$ & $2.51,3.45$ & \\
\hline & & & & $2.51(1 \mathrm{H}, \mathrm{dd}, 16.1,9.7)$ & $36.3,54.3,172.5$ & $2.65,3.45$ & \\
\hline & 3 & $\mathrm{CH}$ & 54.3 & $3.45(\mathrm{ddd}, 9.3,4.5,4.5)$ & $14.7,32.7,36.3,172.5$ & $2.51,2.65,1.80$ & $2.51,2.65$ \\
\hline & 4 & $\mathrm{CH}$ & 36.3 & $1.84-1.76(1 \mathrm{H}, \mathrm{m})$ & $14.7,35.5,54.3$ & $3.45,1.21,1.00$ & \\
\hline & 5 & $\mathrm{CH}_{3}$ & 14.7 & $1.00(3 \mathrm{H}, \mathrm{d}, 6.9)$ & $32.7,36.6,54.3$ & 1.80 & 1.80 \\
\hline & 6 & $\mathrm{CH}_{2}$ & 32.7 & $1.25-1.18(1 \mathrm{H}, \mathrm{m})$ & $14.7,26.0,31.9,36.3$ & $1.80,1.47,1.38$ & \\
\hline & & & & $1.49-1.44(1 \mathrm{H}, \mathrm{m})$ & $14.7,26.0,31.9,36.3$ & 1.21 & \\
\hline & 7 & $\mathrm{CH}_{2}$ & 26.0 & $1.42-1.34(1 \mathrm{H}, \mathrm{m})$ & $32.7,31.9$ & $1.52,2.21,2.07$ & \\
\hline & & & & $1.54-1.49(1 \mathrm{H}, \mathrm{m})$ & $32.7,31.9$ & $1.38,2.07$ & \\
\hline & 8 & $\mathrm{CH}_{2}$ & 31.9 & $2.12-2.03(2 \mathrm{H}, \mathrm{m})$ & $23.3,26.0,120.0,136.5$ & $1.52,1.38$ & \\
\hline & 9 & $\mathrm{C}$ & 136.5 & - & - & - & - \\
\hline & 10 & $\mathrm{CH}_{3}$ & 23.3 & $1.67(3 \mathrm{H}, \mathrm{t}, 1.5)$ & $31.9,120.0,136.9$ & & 5.22 \\
\hline & 11 & $\mathrm{CH}$ & 120.0 & $5.22(1 \mathrm{H}, \mathrm{q}, 6.7)$ & $13.2,23.3,31.9$ & 1.56 & $1.56,1.67$ \\
\hline & 12 & $\mathrm{CH}_{3}$ & 13.2 & $1.56(3 \mathrm{H}, \mathrm{dd}, 6.8,1.3)$ & $23.3,120.0,136.5$ & 5.22 & 5.22 \\
\hline
\end{tabular}




\section{Thaxteramide D}

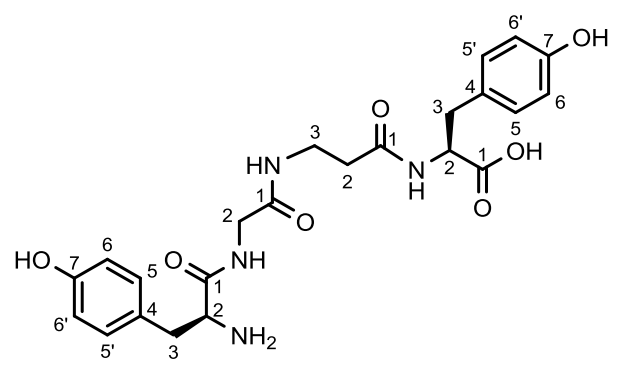

Thaxteramide $\mathrm{D}$ was isolated as described in procedure $\mathrm{B}$ and purified using system SP4. White solid, $0.3 \mathrm{mg},{ }^{1} \mathrm{H}$ NMR (700 MHz, $\mathrm{CD}_{3} \mathrm{OD}$ ) and ${ }^{13} \mathrm{C}$ NMR as described in NMR table S6; HRMS (ESI+): $m / z$ calcd for $\mathrm{C}_{23} \mathrm{H}_{28} \mathrm{~N}_{4} \mathrm{O}_{7}+\mathrm{H}^{+}: 473.20308\left[M+\mathrm{H}^{+}\right]$, found: 473.20352, $\Delta \mathrm{m} 0.93$ ppm; HPLC $t_{\mathrm{R}}=2.38$ (purity $=94 \%$; System A).

Table S6: NMR data of Thaxteramid D in $\mathrm{CD}_{3} \mathrm{OD}$

\begin{tabular}{|c|c|c|c|c|c|c|}
\hline $\begin{array}{c}\text { Partial } \\
\text { structure }\end{array}$ & $\mathbf{C}$ & Type & $\boldsymbol{\delta}_{\mathrm{C}}$ & $\delta_{\mathbf{H}}($ mult., $J$ in $\mathbf{H z})$ & НMBC & COSY \\
\hline & 1 & $\mathrm{CO}$ & 179.0 & - & - & - \\
\hline \multirow[t]{8}{*}{ Tyrosine } & 2 & $\mathrm{CH}$ & 57.6 & $4.41(1 \mathrm{H}, \mathrm{dd}, 10.0,4.1)$ & $37.7,130.6,173.9,179.0$ & $3.15,2.76$ \\
\hline & 3 & $\mathrm{CH}_{2}$ & 37.7 & $3.15(1 \mathrm{H}, \mathrm{dd}, 14.2,4.0)$ & $57.6,130.6,131.1,179.0$ & $4.41,2.76$ \\
\hline & & & & $2.76(1 \mathrm{H}, \mathrm{dd}, 14.2,10.1)$ & $57.6,130.6,131.1,179.0$ & $4.41,3.15$ \\
\hline & 4 & $\mathrm{C}$ & 130.6 & - & - & - \\
\hline & 5,5 & $\mathrm{CH}$ & 131.1 & $7.06(2 \mathrm{H}, \mathrm{d}, 8.5)$ & $37.7,115.7,156.0$ & 7.06 \\
\hline & 6,6 & $\mathrm{CH}$ & 115.7 & $6.67(2 \mathrm{H}, \mathrm{d}, 8.4)$ & $131.1,156.0$ & 6.67 \\
\hline & 7 & $\mathrm{C}$ & 156.0 & - & - & - \\
\hline & 1 & $\mathrm{CO}$ & 174.1 & - & - & - \\
\hline \multirow[t]{5}{*}{$\beta$-Alanine } & 2 & $\mathrm{CH}_{2}$ & 36.8 & $2.36(1 \mathrm{H}, \mathrm{ddd}, 13.6,8.3,4.7)$ & $37.1,174.1$ & $2.18,3.53,3.44$ \\
\hline & & & & $2.18(1 \mathrm{H}, \mathrm{ddd}, 13.8,7.3,4.4)$ & $37.1,174.1$ & $2.36,3.53,3.44$ \\
\hline & 3 & $\mathrm{CH}_{2}$ & 37.1 & $3.53(1 \mathrm{H}, \mathrm{ddd}, 13.8,7.5,4.6)$ & 36.8174 .1 & $2.36,2.18,3.44$ \\
\hline & & & & $3.44(1 \mathrm{H}, \mathrm{ddd}, 13.4,8.2,4.4)$ & $36.8,174.1$ & $2.36,2.18,3.53$ \\
\hline & 1 & $\mathrm{CO}$ & 170.9 & - & - & - \\
\hline \multirow[t]{3}{*}{ Glycine } & 2 & $\mathrm{CH}_{2}$ & 43.4 & $4.04(1 \mathrm{H}, \mathrm{d}, 16.9)$ & 170.9 & 3.50 \\
\hline & & & & $3.50(1 \mathrm{H}, \mathrm{d}, 16.9)$ & 170.9 & 4.04 \\
\hline & 1 & $\mathrm{CO}$ & 170.9 & - & - & - \\
\hline \multirow[t]{7}{*}{ Tyrosine } & 2 & $\mathrm{CH}$ & 56.0 & $4.01(1 \mathrm{H}, \mathrm{t}, 7.5)$ & $37.5,126.1,170.9$ & $3.06,2.95$ \\
\hline & 3 & $\mathrm{CH}_{2}$ & 37.5 & $3.06(1 \mathrm{H}, \mathrm{dd}, 13.9,7.3)$ & $56.0,126.1,131.1,170.9$ & $4.01,2.95$ \\
\hline & & & & $2.95(1 \mathrm{H}, \mathrm{dd}, 13.9,7.7)$ & $56.0,126.1,131.1,170.9$ & $4.01,3.06$ \\
\hline & 4 & $\mathrm{C}$ & 126.1 & - & - & - \\
\hline & 5,5 & $\mathrm{CH}$ & 131.1 & $7.08(2 \mathrm{H}, \mathrm{d}, 8.5)$ & $37.5,116.5,158.1$ & 7.08 \\
\hline & 6,6 & $\mathrm{CH}$ & 116.5 & $6.77(2 \mathrm{H}, \mathrm{d}, 8.5)$ & $126.1,158.1$ & 6.77 \\
\hline & 7 & $\mathrm{C}$ & 158.1 & - & - & - \\
\hline
\end{tabular}




\section{Cyclic Thaxteramide A1}

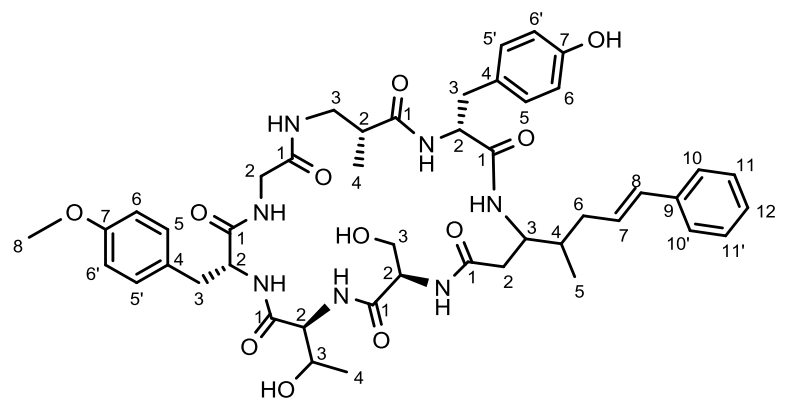

Cyc-Thaxteramide A1 was synthesised as described in procedure E starting from ThxA1 (2.2 $\mathrm{mg}$ ) and purified using system SP3. White solid, $1.7 \mathrm{mg}, 80 \%$ yield. ${ }^{1} \mathrm{H}$ NMR (700 MHz, $\mathrm{CD}_{3} \mathrm{OD}$ ) and ${ }^{13} \mathrm{C}$ NMR as described in NMR table S7; HRMS (ESI+): $\mathrm{m} / z$ calcd for $\mathrm{C}_{46} \mathrm{H}_{59} \mathrm{~N}_{7} \mathrm{O}_{11}+\mathrm{H}^{+}: 886.43453\left[M+\mathrm{H}^{+}\right]$, found: 886.43522, $\Delta \mathrm{m} 0.78 \mathrm{ppm}$; HPLC $t_{\mathrm{R}}=8.61$ (purity $=97 \%$; System A).

Table S7: NMR data of Cyc-Thaxteramide A1 in $\mathrm{CD}_{3} \mathrm{OD}$

\begin{tabular}{|c|c|c|c|c|c|c|}
\hline $\begin{array}{c}\text { Partial } \\
\text { structure }\end{array}$ & $\mathbf{C}$ & Type & $\boldsymbol{\delta}_{\mathrm{C}}$ & $\delta_{H}$ (mult., $J$ in $\mathbf{H z}$ ) & HMBC & COSY \\
\hline \multirow{8}{*}{ Tyrosine } & 1 & $\mathrm{CO}$ & 172.8 & - & - & - \\
\hline & 2 & $\mathrm{CH}$ & 56.1 & $4.56(1 \mathrm{H}, \mathrm{dd}, 8.2,7.5)$ & $38.0,172.8$ & $3.03,2.78$ \\
\hline & 3 & $\mathrm{CH}_{2}$ & 38.0 & $3.03(1 \mathrm{H}, \mathrm{dd}, 13.7,7.4)$ & $56.1,128.6,131.1,172.8$ & $4.56,2.78$ \\
\hline & & & & $2.78(1 \mathrm{H}, \mathrm{dd}, 13.7,8.5)$ & $56.1,128.6,131.1,172.8$ & $4.56,3.03$ \\
\hline & 4 & $\mathrm{C}$ & 128.6 & - & - & - \\
\hline & 5,5 & $\mathrm{CH}$ & 131.1 & $7.07(2 \mathrm{H}, \mathrm{d}, 8.4)$ & $157.0,38.0$ & 6.70 \\
\hline & 6,6 & $\mathrm{CH}$ & 116.0 & $6.70(2 \mathrm{H}, \mathrm{d}, 8.5)$ & $128.6,157.0$ & 7.07 \\
\hline & 7 & $\mathrm{C}$ & 157.0 & - & - & - \\
\hline \multirow{5}{*}{$\begin{array}{l}\text { Methyl- } \beta \text { - } \\
\text { Alanine }\end{array}$} & 1 & $\mathrm{CO}$ & 177.1 & - & - & - \\
\hline & 2 & $\mathrm{CH}$ & 41.0 & $2.62(1 \mathrm{H}, \mathrm{d}, 6.7)^{*}$ & & $3.30,3.18,0.94$ \\
\hline & 3 & $\mathrm{CH}_{2}$ & 43.5 & $3.30(1 \mathrm{H}, \mathrm{dd}, 13.4,9.4)^{\#}$ & $41.0,171.3,177.1$ & $3.18,2.62$ \\
\hline & & & & $3.18(1 \mathrm{H}, \mathrm{dd}, 13.4,4.6)$ & $15.0,41.0,171.3,177.1$ & $3.30,2.62$ \\
\hline & 4 & $\mathrm{CH}_{3}$ & 15.0 & $0.94(3 \mathrm{H}, \mathrm{d}, 6.9)$ & $41.0,43.5,177.1$ & 2.62 \\
\hline \multirow{3}{*}{ Glycine } & 1 & $\mathrm{CO}$ & 171.3 & - & - & - \\
\hline & 2 & $\mathrm{CH}_{2}$ & 43.5 & $3.93(1 \mathrm{H}, \mathrm{d}, 16.6)$ & $171.3,173.8$ & 3.84 \\
\hline & & & & $3.84(1 \mathrm{H}, \mathrm{d}, 16.5)$ & $171.3,173.8$ & 3.93 \\
\hline
\end{tabular}

* Overlapped signals

\# Signal under solvent peak 
Table S7: NMR data of Cyc-Thaxteramide A1 in $\mathrm{CD}_{3} \mathrm{OD}$ (continued)

\begin{tabular}{|c|c|c|c|c|c|c|}
\hline $\begin{array}{c}\text { Partial } \\
\text { structure }\end{array}$ & $\mathbf{C}$ & Type & $\boldsymbol{\delta}_{\mathrm{C}}$ & $\delta_{\mathbf{H}}($ mult., $J$ in $\mathbf{H z})$ & HMBC & COSY \\
\hline \multirow{10}{*}{$\begin{array}{l}\text { O-Methyl- } \\
\text { Tyrosine }\end{array}$} & 1 & $\mathrm{CO}$ & 173.8 & - & - & - \\
\hline & 2 & $\mathrm{CH}$ & 57.6 & $4.42(1 \mathrm{H}, \mathrm{dd}, 10.3,4.5)$ & $37.0,130.3,172.8,173.8$ & $3.05,2.89$ \\
\hline & 3 & $\mathrm{CH}_{2}$ & 37.0 & $3.05(1 \mathrm{H}, \mathrm{dd}, 13.7,4.7)$ & $57.6,130.3,131.0,173.8$ & $4.42,2.89$ \\
\hline & & & & $2.89(1 \mathrm{H}, \mathrm{dd}, 13.9,10.4)$ & $57.6,130.3,131.0,173.8$ & $4.42,3.05$ \\
\hline & 4 & $\mathrm{C}$ & 130.3 & - & - & - \\
\hline & 5,5 & $\mathrm{CH}$ & 131.0 & $7.17(2 \mathrm{H}, \mathrm{d}, 8.5)^{*}$ & $159.7,37.0$ & 6.81 \\
\hline & 6,6 & $\mathrm{CH}$ & 114.6 & $6.81(2 \mathrm{H}, \mathrm{d}, 8.5)$ & $130.3,159.7$ & 7.17 \\
\hline & 7 & $\mathrm{C}$ & 159.7 & - & - & - \\
\hline & 8 & $\mathrm{OCH}_{3}$ & 55.3 & $3.75(3 \mathrm{H}, \mathrm{s})$ & 159.7 & \\
\hline & 1 & $\mathrm{CO}$ & 172.8 & - & - & - \\
\hline \multirow[t]{5}{*}{ Threonine } & 2 & $\mathrm{CH}$ & 60.1 & $4.09(1 \mathrm{H}, \mathrm{d}, 3.4)$ & $67.1,172.8$ & 4.20 \\
\hline & 3 & $\mathrm{CH}$ & 67.1 & $4.20(1 \mathrm{H}, \mathrm{dq}, 6.3,3.5)$ & & $4.09,1.05$ \\
\hline & 4 & $\mathrm{CH}_{3}$ & 20.2 & $1.05(3 \mathrm{H}, \mathrm{d}, 6.3)$ & $60.1,67.1$ & 4.20 \\
\hline & 1 & $\mathrm{CO}$ & 172.4 & - & - & - \\
\hline & 2 & $\mathrm{CH}$ & 58.7 & $4.23(1 \mathrm{H}, \mathrm{t}, 4.6)$ & $61.8,172.4$ & $3.87,3.90$ \\
\hline \multirow[t]{3}{*}{ Serine } & 3 & $\mathrm{CH}_{2}$ & 61.8 & $3.90(1 \mathrm{H}, \mathrm{dd}, 11.4,5.1)$ & $58.7,172.4$ & $4.23,3.87$ \\
\hline & & & & $3.87(1 \mathrm{H}, \mathrm{dd}, 11.2,4.2)$ & $58.7,172.4$ & $4.23,3.90$ \\
\hline & 1 & $\mathrm{CO}$ & 175.1 & - & - & - \\
\hline \multirow[t]{12}{*}{ Lipid chain } & 2 & $\mathrm{CH}_{2}$ & 38.0 & $2.62(1 \mathrm{H}, \mathrm{d}, 6.7)^{*}$ & $36.7,53.2,175.1$ & 4.25 \\
\hline & 3 & $\mathrm{CH}$ & 51.9 & $4.25(1 \mathrm{H}, \mathrm{q}, 6.3)$ & $16.1,38.0,37.6,172.8,175.1$ & $2.62,1.79$ \\
\hline & 4 & $\mathrm{CH}$ & 37.6 & $1.79(1 \mathrm{H}, \mathrm{dt}, 12.9,6.5)$ & 51.9 & $0.84,1.84,4.25$ \\
\hline & 5 & $\mathrm{CH}_{3}$ & 16.1 & $0.84(3 \mathrm{H}, \mathrm{d}, 6.6)$ & $36.9,37.6,51.9$ & 1.79 \\
\hline & 6 & $\mathrm{CH}_{2}$ & 36.9 & $2.20(1 \mathrm{H}, \mathrm{dt}, 13.4,6.7)$ & $16.1,129.3,132.7$ & $6.17,1.84$ \\
\hline & & & & $1.84(1 \mathrm{H}, \mathrm{dt}, 14.7,7.4)$ & $37.6,51.9,129.3,132.7$ & $6.17,2.20$ \\
\hline & 7 & $\mathrm{CH}$ & 129.3 & $6.17(1 \mathrm{H}, \mathrm{dt}, 15.8,7.3)$ & 138.9 & $6.38,2.20,1.84$ \\
\hline & 8 & $\mathrm{CH}$ & 132.7 & $6.38(1 \mathrm{H}, \mathrm{d}, 15.8)$ & $126.8,36.9$ & 6.17 \\
\hline & 9 & $\mathrm{C}$ & 138.9 & - & - & - \\
\hline & $10,10^{\prime}$ & $\mathrm{CH}$ & 126.8 & $7.33(2 \mathrm{H}, \mathrm{d}, 7.4)$ & $127.7,132.7$ & 7.24 \\
\hline & $11,11^{\prime}$ & $\mathrm{CH}$ & 129.2 & $7.24(2 \mathrm{H}, \mathrm{t}, 7.7)$ & 138.9 & $7.33,7.16$ \\
\hline & 12 & $\mathrm{CH}$ & 127.7 & $7.16(1 \mathrm{H})^{*}$ & 126.8 & 7.24 \\
\hline
\end{tabular}

* Overlapped signals 


\section{Cyclic Thaxteramide B1}

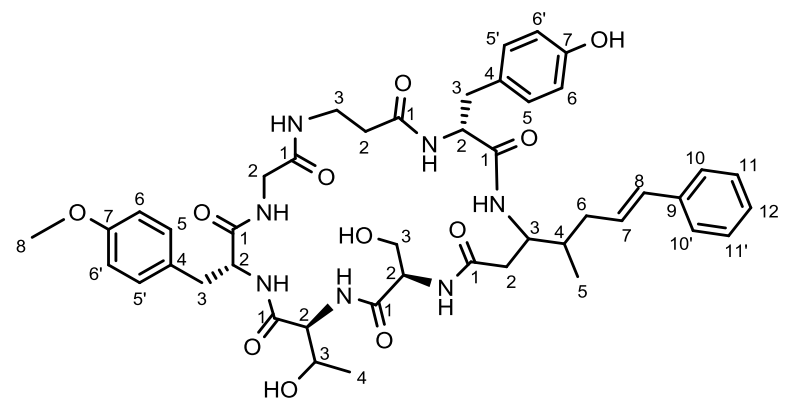

Cyc-Thaxteramide B1 was synthesised as described in procedure E starting from ThxB1 (0.6 $\mathrm{mg}$ ) and purified using system SP3. White solid, $0.4 \mathrm{mg}, 70 \%$ yield. ${ }^{1} \mathrm{H}$ NMR $(700 \mathrm{MHz}$, $\mathrm{CD}_{3} \mathrm{OD}$ ) and ${ }^{13} \mathrm{C}$ NMR as described in NMR table S8; HRMS (ESI+): $\mathrm{m} / \mathrm{z}$ calcd for $\mathrm{C}_{45} \mathrm{H}_{57} \mathrm{~N}_{7} \mathrm{O}_{11}+\mathrm{H}^{+}: 872.41888\left[M+\mathrm{H}^{+}\right]$, found: 872.41912, $\Delta \mathrm{m} 0.28$ ppm; HPLC $t_{\mathrm{R}}=8.34$ (purity $=99 \%$; System A).

Table S8: NMR data of Cyc-Thaxteramide $\mathrm{B} 1$ in $\mathrm{CD}_{3} \mathrm{OD}$

\begin{tabular}{|c|c|c|c|c|c|c|}
\hline $\begin{array}{c}\text { Partial } \\
\text { structure }\end{array}$ & $\mathbf{C}$ & Type & $\boldsymbol{\delta}_{\mathrm{C}}$ & $\delta_{\mathbf{H}}($ mult., $J$ in $\mathbf{H z})$ & HMBC & COSY \\
\hline \multirow{8}{*}{ Tyrosine } & 1 & $\mathrm{CO}$ & 172.9 & - & - & - \\
\hline & 2 & $\mathrm{CH}$ & 57.1 & $4.42(1 \mathrm{H}, \mathrm{dd}, 8.7,6.9)$ & $37.5,128.1,172.9$ & $2.93,2.84$ \\
\hline & 3 & $\mathrm{CH}_{2}$ & 37.5 & $2.93(1 \mathrm{H}, \mathrm{dd}, 13.5,8.9)$ & $57.1,128.1,131.1$ & 4.422 .84 \\
\hline & & & & $2.84(1 \mathrm{H}, \mathrm{dd}, 13.5,7.2)$ & $57.1,128.1,131.1,172.9$ & $4.42,2.93$ \\
\hline & 4 & $\mathrm{C}$ & 128.1 & - & - & - \\
\hline & 5,5 & $\mathrm{CH}$ & 131.1 & $7.06(2 \mathrm{H}, \mathrm{d}, 8.5)$ & $157.2,37.5$ & 6.69 \\
\hline & 6,6 & $\mathrm{CH}$ & 116.1 & $6.69(2 \mathrm{H}, \mathrm{d}, 8.5)$ & $128.1,157.2$ & 7.06 \\
\hline & 7 & $\mathrm{C}$ & 157.2 & - & - & - \\
\hline \multirow{5}{*}{$\begin{array}{l}\text { Methyl- } \beta \text { - } \\
\text { Alanine }\end{array}$} & 1 & $\mathrm{CO}$ & 173.6 & - & - & - \\
\hline & 2 & $\mathrm{CH}_{2}$ & 35.6 & $2.48(1 \mathrm{H}, \mathrm{dd}, 15.4,7.3)^{*}$ & 173.6 & $2.39,3.51,3.42$ \\
\hline & & & & $2.39(1 \mathrm{H}, \mathrm{ddd}, 15.2,7.7,4.4)$ & 173.6 & $2.48,3.51,3.42$ \\
\hline & 3 & $\mathrm{CH}_{2}$ & 36.5 & 3.54-3.48 (1H, m) & 173.6 & $2.39,2.48,3.42$ \\
\hline & & & & $3.44-3.41(1 \mathrm{H}, \mathrm{m})$ & 173.6 & $2.39,2.48,3.51$ \\
\hline \multirow{3}{*}{ Glycine } & 1 & $\mathrm{CO}$ & 171.5 & - & - & - \\
\hline & 2 & $\mathrm{CH}_{2}$ & 43.7 & $3.97(1 \mathrm{H}, \mathrm{d}, 16.6)^{*}$ & $171.5,173.8$ & 3.67 \\
\hline & & & & $3.67(1 \mathrm{H}, \mathrm{d}, 16.5)$ & $171.5,173.8$ & 3.97 \\
\hline \multirow{9}{*}{$\begin{array}{l}\text { O-Methyl- } \\
\text { Tyrosine }\end{array}$} & 1 & $\mathrm{CO}$ & 173.8 & - & - & - \\
\hline & 2 & $\mathrm{CH}$ & 57.4 & $4.43(1 \mathrm{H}, \mathrm{dd}, 10.3,5.7)$ & $36.7,130.1,173.8$ & $3.17,2.85$ \\
\hline & 3 & $\mathrm{CH}_{2}$ & 36.7 & $3.17(1 \mathrm{H}, \mathrm{dd}, 14.3,5.5)$ & $57.4,130.1,131.1$ & $4.43,2.85$ \\
\hline & & & & $2.85(1 \mathrm{H}, \mathrm{dd}, 13.9,10.0)$ & $57.4,130.1,131.1,173.8$ & $4.43,3.17$ \\
\hline & 4 & $\mathrm{C}$ & 130.1 & - & - & - \\
\hline & 5,5 & $\mathrm{CH}$ & 131.1 & $7.16(2 \mathrm{H}, \mathrm{d}, 8.7)^{*}$ & $36.7,159.9$ & 6.83 \\
\hline & $6,6^{\prime}$ & $\mathrm{CH}$ & 114.7 & $6.83(2 \mathrm{H}, \mathrm{d}, 8.7)$ & $130.1,159.9$ & 7.16 \\
\hline & 7 & $\mathrm{C}$ & 159.9 & - & - & - \\
\hline & 8 & $\mathrm{OCH}_{3}$ & 55.3 & $3.75(3 \mathrm{H}, \mathrm{s})$ & 159.9 & \\
\hline
\end{tabular}

* Overlapped signals 
Table S8: NMR data of Cyc-Thaxteramide $\mathrm{B} 1$ in $\mathrm{CD}_{3} \mathrm{OD}$ (continued)

\begin{tabular}{|c|c|c|c|c|c|c|}
\hline $\begin{array}{c}\text { Partial } \\
\text { structure }\end{array}$ & $\mathbf{C}$ & Type & $\boldsymbol{\delta}_{\mathrm{C}}$ & $\delta_{\mathbf{H}}$ (mult., $J$ in $\mathrm{Hz}$ ) & HМBC & COSY \\
\hline \multirow{4}{*}{ Threonine } & 1 & $\mathrm{CO}$ & 172.9 & - & - & - \\
\hline & 2 & $\mathrm{CH}$ & 60.5 & $4.13(1 \mathrm{H}, \mathrm{d}, 4.9)$ & $20.0,67.9,172.9$ & 4.00 \\
\hline & 3 & $\mathrm{CH}$ & 67.9 & $4.00(1 \mathrm{H}, \mathrm{dd}, 6.2,5.1)$ & & $4.13,0.95$ \\
\hline & 4 & $\mathrm{CH}_{3}$ & 20.0 & $0.95(3 \mathrm{H}, \mathrm{d}, 6.4)$ & $60.5,67.9$ & 4.00 \\
\hline \multirow{4}{*}{ Serine } & 1 & $\mathrm{CO}$ & 172.8 & - & - & - \\
\hline & 2 & $\mathrm{CH}$ & 57.3 & $4.34(1 \mathrm{H}, \mathrm{dd}, 6.5,5.2)$ & $61.8,172.8$ & $3.87,3.83$ \\
\hline & 3 & $\mathrm{CH}_{2}$ & 61.8 & $3.87(1 \mathrm{H}, \mathrm{dd}, 11.3,5.0)$ & 172.4 & $4.34,3.83$ \\
\hline & & & & $3.83(1 \mathrm{H}, \mathrm{dd}, 11.1,6.5)$ & $57.3,172.8$ & $4.34,3.87$ \\
\hline \multirow{14}{*}{ Lipid chain } & 1 & $\mathrm{CO}$ & 173.9 & - & - & - \\
\hline & 2 & $\mathrm{CH}_{2}$ & 38.2 & $2.58(1 \mathrm{H}, \mathrm{dd}, 14.3,4.6)$ & $36.8,52.5,173.9$ & $3.97,2.47$ \\
\hline & & & & $2.47(1 \mathrm{H}, \mathrm{dd}, 14.6,7.0)^{*}$ & $36.8,52.5,173.9$ & $2.58,3.97$ \\
\hline & 3 & $\mathrm{CH}$ & 52.5 & $3.99-3.94(1 \mathrm{H}, \mathrm{m})^{*}$ & 37.1 & $2.58,2.47,1.70$ \\
\hline & 4 & $\mathrm{CH}$ & 36.8 & $1.74-1.67(1 \mathrm{H}, \mathrm{m})$ & & $0.85,3.97$ \\
\hline & 5 & $\mathrm{CH}_{3}$ & 16.3 & $0.85(3 \mathrm{H}, \mathrm{d}, 6.6)$ & $36.8,37.1,52.5$ & 1.70 \\
\hline & 6 & $\mathrm{CH}_{2}$ & 37.1 & $2.0-1.95(1 \mathrm{H}, \mathrm{m})$ & & $1.65,6.08$ \\
\hline & & & & $1.68-1.63(1 \mathrm{H}, \mathrm{m})$ & $16.3,36.8,129.2,132.7$ & $1.98,6.08$ \\
\hline & 7 & $\mathrm{CH}$ & 129.2 & $6.08(1 \mathrm{H}, \mathrm{dt}, 15.7,7.2)$ & 139.0 & $6.31,1.98,1.65$ \\
\hline & 8 & $\mathrm{CH}$ & 132.7 & $6.31(1 \mathrm{H}, \mathrm{d}, 15.6)$ & $126.8,37.1$ & 6.08 \\
\hline & 9 & $\mathrm{C}$ & 139.0 & - & - & - \\
\hline & $10,10^{\prime}$ & $\mathrm{CH}$ & 126.8 & $7.34(2 \mathrm{H}, \mathrm{d}, 7.6)$ & $127.7,132.8$ & 7.25 \\
\hline & 11,11 & $\mathrm{CH}$ & 129.1 & $7.25(2 \mathrm{H}, \mathrm{t}, 7.7)$ & 139.0 & $7.34,7.16$ \\
\hline & 12 & $\mathrm{CH}$ & 127.7 & $7.16(1 \mathrm{H})^{*}$ & 126.8 & 7.25 \\
\hline
\end{tabular}

* Overlapped signals 


\section{Cyclic Thaxteramide $\mathbf{C}$}

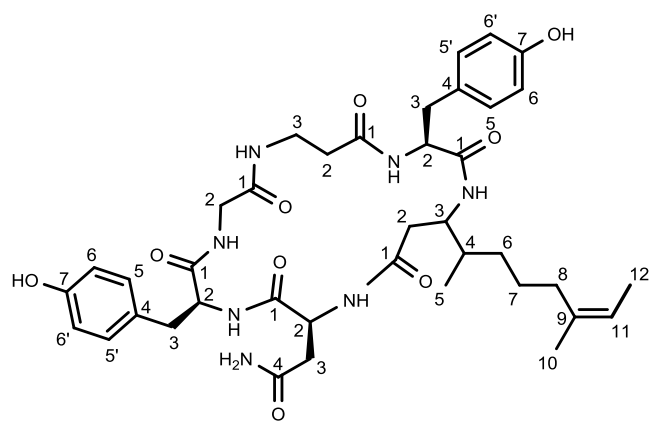

Cyc-Thaxteramide $\mathrm{C}$ was synthesised as described in procedure $\mathrm{E}$ starting from ThxC $(0.6 \mathrm{mg})$ and purified using system SP3. White solid, $0.5 \mathrm{mg}, 80 \%$ yield. ${ }^{1} \mathrm{H}$ NMR $\left(700 \mathrm{MHz}, \mathrm{CD}_{3} \mathrm{OD}\right.$ ) and ${ }^{13} \mathrm{C}$ NMR as described in NMR table S9; HRMS (ESI+): $\mathrm{m} / z$ calcd for $\mathrm{C}_{39} \mathrm{H}_{53} \mathrm{~N}_{7} \mathrm{O}_{9}+\mathrm{H}^{+}$: $764.39775\left[M+\mathrm{H}^{+}\right]$, found: 764.39692, $\Delta \mathrm{m}-1.09 \mathrm{ppm}$; HPLC $t_{\mathrm{R}}=7.90$ (purity $=99 \%$; System A).

Table S9: NMR data of Cyc-Thaxteramide $\mathrm{C}$ in $\mathrm{CD}_{3} \mathrm{OD}$

\begin{tabular}{|c|c|c|c|c|c|c|}
\hline $\begin{array}{c}\text { Partial } \\
\text { structure }\end{array}$ & $\mathbf{C}$ & Type & $\boldsymbol{\delta}_{\mathrm{C}}$ & $\delta_{H}($ mult., $J$ in $\mathrm{Hz})$ & НМBC & COSY \\
\hline & 1 & $\mathrm{CO}$ & 173.5 & - & - & - \\
\hline \multirow[t]{8}{*}{ Tyrosine } & 2 & $\mathrm{CH}$ & 56.4 & $4.53(1 \mathrm{H}, \mathrm{dd}, 8.3,7.2)$ & $38.6,173.1$ & $2.89,2.83$ \\
\hline & 3 & $\mathrm{CH}_{2}$ & 38.6 & $2.89(1 \mathrm{H}, \mathrm{dd}, 13.6,8.5)$ & $56.4,128.4,131.0,173.1$ & $4.53,2.83$ \\
\hline & & & & $2.83(1 \mathrm{H}, \mathrm{dd}, 13.6,6.9)$ & $56.4,128.4,131.0,173.0$ & $4.53,2.89$ \\
\hline & 4 & $\mathrm{C}$ & 128.4 & - & - & - \\
\hline & 5,5 & $\mathrm{CH}$ & 131.0 & $7.04(2 \mathrm{H}, \mathrm{d}, 8.5)$ & $157.1,38.6$ & 7.04 \\
\hline & $6,6^{\prime}$ & $\mathrm{CH}$ & 116.0 & $6.68(2 \mathrm{H}, \mathrm{d}, 8.5)$ & $128.4,157.1$ & 6.68 \\
\hline & 7 & $\mathrm{C}$ & 157.1 & - & - & - \\
\hline & 1 & $\mathrm{CO}$ & 172.8 & - & - & - \\
\hline \multirow[t]{4}{*}{$\beta$-Alanine } & 2 & $\mathrm{CH}_{2}$ & 35.8 & $2.38(1 \mathrm{H}, \mathrm{t}, 5.4)$ & $35.8,172.8$ & $3.53,3.45$ \\
\hline & 3 & $\mathrm{CH}_{2}$ & 35.8 & $3.55-3.51(1 \mathrm{H}, \mathrm{m})$ & $35.8,171.5,172.8$ & $3.45,2.38$ \\
\hline & & & & $3.45(1 \mathrm{H}, \mathrm{dt}, 13.7,5.5)$ & $35.8,171.5,172.8$ & $3.53,2.38$ \\
\hline & 1 & $\mathrm{CO}$ & 171.5 & - & - & - \\
\hline \multirow[t]{3}{*}{ Glycine } & 2 & $\mathrm{CH}_{2}$ & 43.4 & $3.80(1 \mathrm{H}, \mathrm{d}, 17.0)$ & $171.5,174.1$ & 3.76 \\
\hline & & & & $3.76(1 \mathrm{H}, \mathrm{d}, 17.0)$ & $171.5,174.1$ & 3.80 \\
\hline & 1 & $\mathrm{CO}$ & 174.1 & - & - & - \\
\hline \multirow[t]{7}{*}{ Tyrosine } & 2 & $\mathrm{CH}$ & 58.0 & $4.26(1 \mathrm{H}, \mathrm{dd}, 9.1,5.7)$ & $36.1,128.8,174.1$ & $3.08,2.95$ \\
\hline & 3 & $\mathrm{CH}_{2}$ & 36.1 & $3.08(1 \mathrm{H}, \mathrm{dd}, 14.1,5.6)$ & $58.0,128.8,131.0,174.1$ & $4.26,2.95$ \\
\hline & & & & $2.95(1 \mathrm{H}, \mathrm{dd}, 14.0,9.2)$ & $58.0,128.8,131.0,174.1$ & $4.26,3.08$ \\
\hline & 4 & $\mathrm{C}$ & 128.8 & - & - & - \\
\hline & 5,5 & $\mathrm{CH}$ & 131.0 & $7.06(2 \mathrm{H}, \mathrm{d}, 8.5)$ & $36.1,157.1$ & 7.06 \\
\hline & $6,6^{\prime}$ & $\mathrm{CH}$ & 116.0 & $6.71(2 \mathrm{H}, \mathrm{d}, 8.5)$ & $128.8,157.1$ & 6.71 \\
\hline & 7 & $\mathrm{C}$ & 157.1 & - & - & - \\
\hline
\end{tabular}


Table S10: NMR data of Cyc-Thaxteramide $\mathrm{C}$ in $\mathrm{CD}_{3} \mathrm{OD}$ (continued)

\begin{tabular}{|c|c|c|c|c|c|c|}
\hline $\begin{array}{c}\text { Partial } \\
\text { structure }\end{array}$ & $\mathbf{C}$ & Type & $\boldsymbol{\delta}_{\mathrm{C}}$ & $\delta_{\mathrm{H}}($ mult., $J$ in $\mathrm{Hz})$ & HMBC & COSY \\
\hline & 1 & $\mathrm{CO}$ & 173.8 & - & - & - \\
\hline \multirow[t]{5}{*}{ Asparagine } & 2 & $\mathrm{CH}$ & 51.7 & $3.99(1 \mathrm{H}, \mathrm{ddd}, 9.3,5.8,2.9)$ & 173.8 & $2.30,2.36$ \\
\hline & 3 & $\mathrm{CH}_{2}$ & 40.2 & $2.30(1 \mathrm{H}, \mathrm{dd}, 14.2,9.3)$ & $51.7,173.8$ & $2.36,3.99$ \\
\hline & & & & $2.36(1 \mathrm{H}, \mathrm{dd}, 14.3,3.1)$ & $51.7,173.8$ & $2.30,2.99$ \\
\hline & 4 & $\mathrm{CO}$ & 173.8 & - & - & - \\
\hline & 1 & $\mathrm{CO}$ & 175.1 & - & - & - \\
\hline \multirow[t]{12}{*}{ Lipid chain } & 2 & $\mathrm{CH}_{2}$ & 37.5 & $2.63(2 \mathrm{H}, \mathrm{dd}, 6.4,1.9)$ & $52.0,173.5,175.1$ & 4.50 \\
\hline & 3 & $\mathrm{CH}$ & 52.0 & $4.50(1 \mathrm{H}, \mathrm{t}, 6.4)$ & $37.5,173.5,175.1$ & 2.63 \\
\hline & 4 & $\mathrm{CH}$ & 37.9 & $1.51-1.45(1 \mathrm{H}, \mathrm{m})$ & $14.8,34.1,52.0$ & 0.65 \\
\hline & 5 & $\mathrm{CH}_{3}$ & 14.8 & $0.65(3 \mathrm{H}, \mathrm{d}, 6.9)$ & $33.7,34.1,52.0$ & 1.48 \\
\hline & 6 & $\mathrm{CH}_{2}$ & 34.1 & $1.27-1.19(1 \mathrm{H}, \mathrm{m})$ & $14.9,37.9$ & 0.93 \\
\hline & & & & $0.97-0.85(1 \mathrm{H}, \mathrm{m})$ & & 1.23 \\
\hline & 7 & $\mathrm{CH}_{2}$ & 26.0 & $1.41-1.30(2 \mathrm{H}, \mathrm{m})^{*}$ & & \\
\hline & 8 & $\mathrm{CH}_{2}$ & 32.1 & $2.02-1.98(1 \mathrm{H}, \mathrm{m})$ & $23.4,26.0,119.7,136.8$ & 1.35 \\
\hline & 9 & $\mathrm{C}$ & 136.8 & - & - & - \\
\hline & 10 & $\mathrm{CH}_{3}$ & 23.4 & $1.66(3 \mathrm{H}, \mathrm{t}, 1.5)$ & $32.1,119.7,136.8$ & $1.55,5.19$ \\
\hline & 11 & $\mathrm{CH}$ & 119.7 & $5.19(1 \mathrm{H}, \mathrm{q}, 6.2)$ & $13.2,23.4,32.1$ & $1.55,1.66$ \\
\hline & 12 & $\mathrm{CH}_{3}$ & 13.2 & $1.55(3 \mathrm{H}, \mathrm{dd}, 6.7,1.3)$ & $23.5,119.7,136.8$ & $5.19,1.66$ \\
\hline
\end{tabular}

* Overlapped signals 
MS $^{2}$ fragmentation analysis

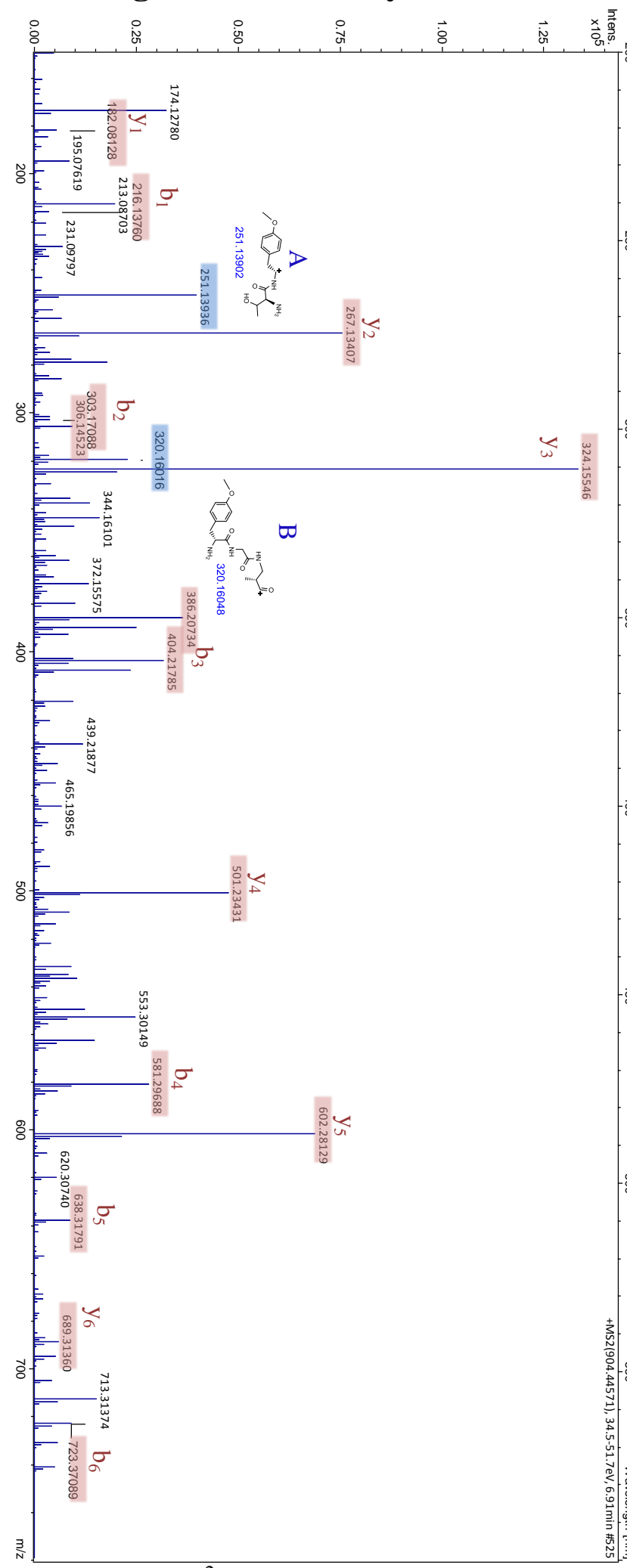

\section{Thaxteramide A1}

Table S10: Identified $\mathrm{MS}^{2}$ fragments of thaxteramide A1

\begin{tabular}{|c|c|c|c|}
\hline Ion & Expected & Found & $\begin{array}{l}\Delta \mathbf{m} \\
(\mathbf{p p m})\end{array}$ \\
\hline $\mathbf{H}^{+}$ & 904.44510 & 904.44577 & 0.74 \\
\hline$-\mathrm{H}_{2} \mathrm{O}$ & 886.43453 & 886.43280 & -1.95 \\
\hline $\mathbf{y}_{6}$ & 689.31408 & 689.31360 & -0.70 \\
\hline $\mathbf{y}_{5}$ & 602.28205 & 602.28129 & -1.26 \\
\hline $\mathbf{y}_{4}$ & 501.23438 & 501.23431 & -0.14 \\
\hline $\mathbf{y}_{\mathbf{3}}$ & 324.15540 & 324.15546 & 0.19 \\
\hline $\mathbf{y}_{3}-\mathbf{H}_{2} \mathbf{O}$ & 306.14483 & 306.14523 & 1.31 \\
\hline $\mathbf{y}_{2}$ & 267.13393 & 267.13407 & 0.52 \\
\hline $\mathbf{y}_{1}$ & 182.08117 & 182.08128 & 0.60 \\
\hline$b_{6}$ & 723.37120 & 723.37089 & -0.43 \\
\hline $\mathbf{b}_{5}$ & 638.31844 & 638.31791 & -0.83 \\
\hline $\mathbf{b}_{4}$ & 581.29698 & 581.29688 & -0.17 \\
\hline $\mathbf{b}_{3}$ & 404.21800 & 404.21785 & -0.37 \\
\hline$b_{3}-H_{2} O$ & 386.20743 & 386.20734 & 0.23 \\
\hline $\mathbf{b}_{2}$ & 303.17032 & 303.17088 & 1.85 \\
\hline$b_{1}$ & 216.13829 & 216.13760 & -3.19 \\
\hline $\mathbf{A}$ & 251.13902 & 251.13936 & 1.35 \\
\hline B & 320.16048 & 320.16016 & -1.00 \\
\hline
\end{tabular}

Figure S1: $\mathrm{MS}^{2}$ fragmentation spectrum of thaxteramide $\mathrm{A} 1$ 


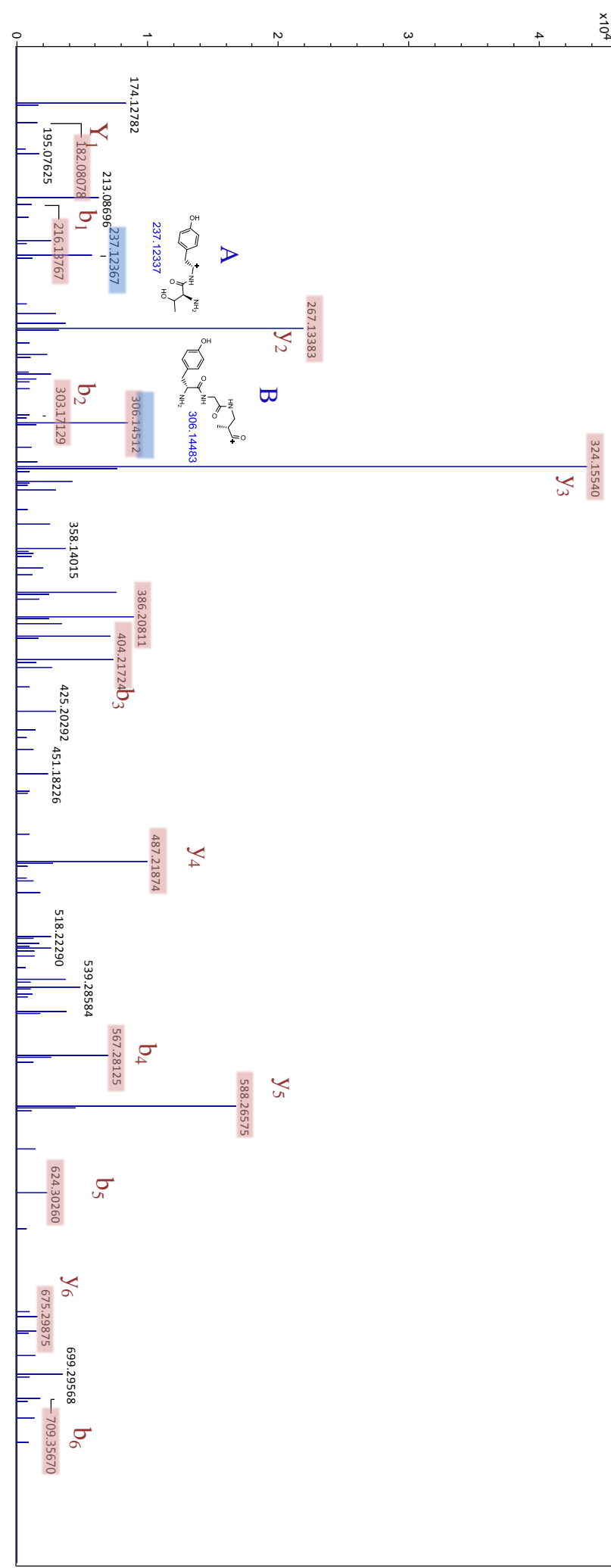

Thaxteramide A2

Table S11: Identified $\mathrm{MS}^{2}$ fragments of thaxteramide A2

\begin{tabular}{|c|c|c|c|}
\hline Ion & Expected & Found & $\begin{array}{l}\Delta \mathbf{m} \\
(\mathbf{p p m})\end{array}$ \\
\hline $\mathbf{H}^{+}$ & 890.42945 & 890.42935 & -0.11 \\
\hline$-\mathrm{H}_{2} \mathrm{O}$ & 872.41888 & 872.41582 & -3.51 \\
\hline $\mathbf{y}_{6}$ & 675.29843 & 675.29875 & 0.47 \\
\hline $\mathbf{y}_{5}$ & 588.26640 & 588.26575 & -1.10 \\
\hline $\mathbf{y}_{4}$ & 487.21873 & 487.21874 & 0.02 \\
\hline $\mathbf{y}_{3}$ & 324.15540 & 324.15540 & 0.00 \\
\hline $\mathbf{y}_{3}-\mathbf{H}_{2} \mathrm{O}$ & 306.14483 & 306.14512 & 0.95 \\
\hline $\mathbf{y}_{2}$ & 267.13393 & 267.13383 & -0.37 \\
\hline $\mathbf{y}_{1}$ & 182.08117 & 182.08078 & -2.14 \\
\hline$b_{6}$ & 709.35555 & 709.35670 & 1.62 \\
\hline$b_{5}$ & 624.30279 & 624.30260 & -0.30 \\
\hline $\mathbf{b}_{4}$ & 567.28133 & 567.28125 & -0.14 \\
\hline $\mathbf{b}_{3}$ & 404.21800 & 404.21724 & -1.88 \\
\hline$b_{3}-H_{2} O$ & 386.20743 & 386.20811 & 1.76 \\
\hline $\mathbf{b}_{2}$ & 303.17032 & 303.17129 & 3.20 \\
\hline$b_{1}$ & 216.13829 & 216.13767 & -2.87 \\
\hline $\mathbf{A}$ & 237.12337 & 237.12367 & 1.27 \\
\hline B & 306.14483 & 306.14512 & 0.95 \\
\hline
\end{tabular}

Figure S2: $\mathrm{MS}^{2}$ fragmentation spectrum of thaxteramide $\mathrm{A} 2$ 


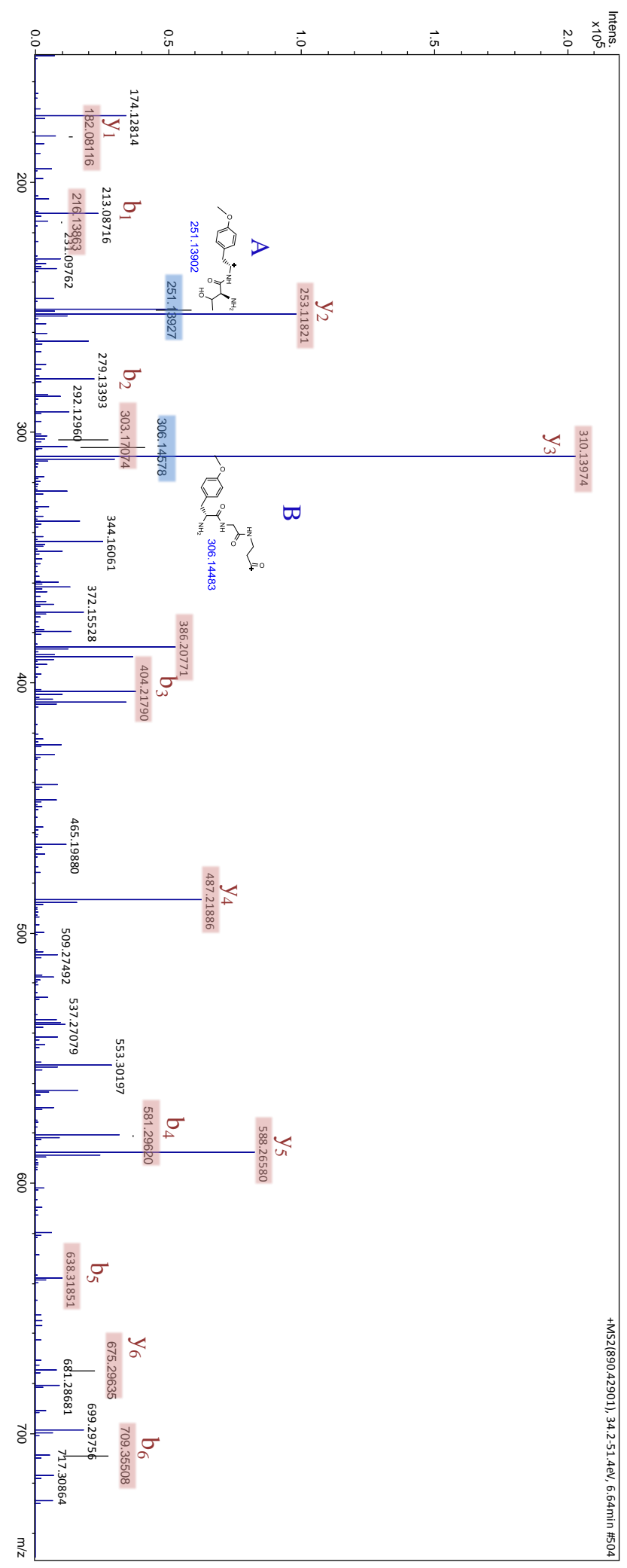

\section{Thaxteramide B1}

Table S12: Identified $\mathrm{MS}^{2}$ fragments of thaxteramide B1

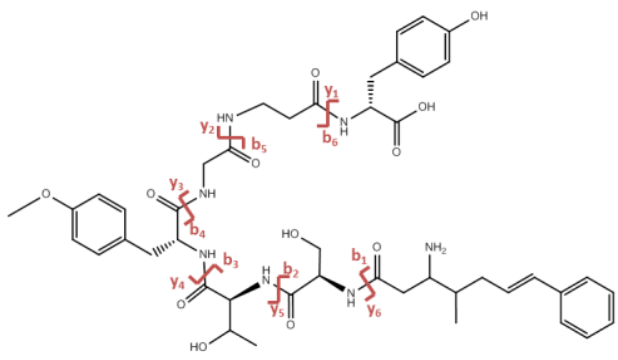

\begin{tabular}{llll}
\hline Ion & Expected & Found & $\begin{array}{l}\Delta \mathbf{m} \\
(\mathbf{p p m})\end{array}$ \\
\hline $\mathbf{H}^{+}$ & 890.42945 & 890.42919 & -0.29 \\
$\mathbf{- H}_{\mathbf{2}} \mathbf{O}$ & 872.41888 & 872.41758 & -1.49 \\
$\mathbf{y}_{\mathbf{6}}$ & 675.29843 & 675.29635 & -3.08 \\
$\mathbf{y}_{\mathbf{5}}$ & 588.26640 & 588.26580 & -1.02 \\
$\mathbf{y}_{\mathbf{4}}$ & 487.21873 & 487.21886 & -0.27 \\
$\mathbf{y}_{\mathbf{3}}$ & 310.13975 & 310.13974 & 0.03 \\
$\mathbf{y}_{\mathbf{2}}$ & 253.11828 & 253.11821 & 0.28 \\
$\mathbf{y}_{\mathbf{1}}$ & 182.08117 & 182.08116 & 0.05 \\
$\mathbf{b}_{\mathbf{6}}$ & 709.35555 & 709.35508 & -0.66 \\
$\mathbf{b}_{\mathbf{5}}$ & 638.31844 & 638.31851 & 0.11 \\
$\mathbf{b}_{\mathbf{4}}$ & 581.29698 & 581.29620 & -1.34 \\
$\mathbf{b}_{\mathbf{3}}$ & 404.21800 & 404.21790 & -0.25 \\
$\mathbf{b}_{\mathbf{3}}-\mathbf{H}_{\mathbf{2}} \mathbf{O}$ & 386.20743 & 386.20771 & 0.72 \\
$\mathbf{b}_{\mathbf{2}}$ & 303.17032 & 303.17074 & 1.39 \\
$\mathbf{b}_{\mathbf{1}}$ & 216.13829 & 216.13863 & 1.57 \\
$\mathbf{A}$ & 251.13902 & 251.13927 & 1.00 \\
$\mathbf{B}$ & 306.14483 & 306.14578 & 3.10 \\
\hline
\end{tabular}

Figure S3: $\mathrm{MS}^{2}$ fragmentation spectrum of thaxteramide B1 


\section{Thaxteramide B2}

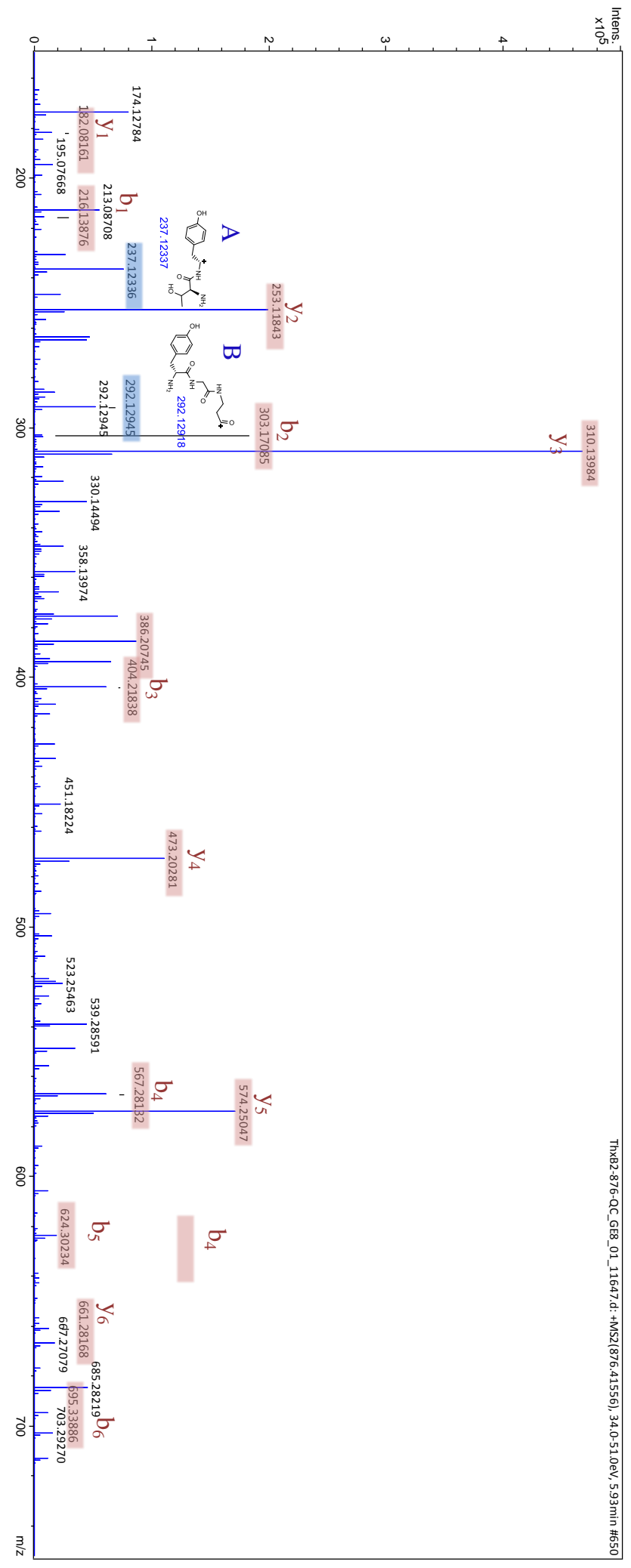

Table S13: Identified $\mathrm{MS}^{2}$ fragments of thaxteramide B2

\begin{tabular}{|c|c|c|c|}
\hline Ion & Expected & Found & $\begin{array}{l}\Delta \mathrm{m} \\
(\mathrm{ppm})\end{array}$ \\
\hline$\overline{\mathbf{H}^{+}}$ & 876.41380 & 876.41491 & 1.27 \\
\hline $\mathbf{y}_{6}$ & 661.28278 & 661.28168 & -1.66 \\
\hline $\mathbf{y}_{5}$ & 574.25075 & 574.25047 & -0.49 \\
\hline $\mathbf{y}_{4}$ & 473.20308 & 473.20281 & -0.57 \\
\hline $\mathbf{y}_{3}$ & 310.13975 & 310.13984 & 0.29 \\
\hline $\mathbf{y}_{2}$ & 253.11828 & 253.11843 & 0.59 \\
\hline $\mathbf{y}_{1}$ & 182.08117 & 182.08161 & 2.42 \\
\hline$b_{6}$ & 695.33990 & 695.33886 & -1.50 \\
\hline$b_{5}$ & 624.30279 & 624.30234 & -0.72 \\
\hline $\mathbf{b}_{4}$ & 567.29698 & 567.281322 & -27.60 \\
\hline $\mathbf{b}_{3}$ & 404.21800 & 404.21838 & 0.94 \\
\hline $\mathbf{b}_{3}-\mathbf{H}_{2} \mathrm{O}$ & 386.20743 & 386.20745 & 0.05 \\
\hline $\mathbf{b}_{2}$ & 303.17032 & 303.17085 & 1.75 \\
\hline$b_{1}$ & 216.13829 & 216.13876 & 2.17 \\
\hline $\mathbf{A}$ & 237.12337 & 237.12336 & -0.04 \\
\hline B & 292.12918 & 292.12945 & 0.92 \\
\hline
\end{tabular}

Figure S4: $\mathrm{MS}^{2}$ fragmentation spectrum of Thaxteramide B2 


\section{Thaxteramide C}

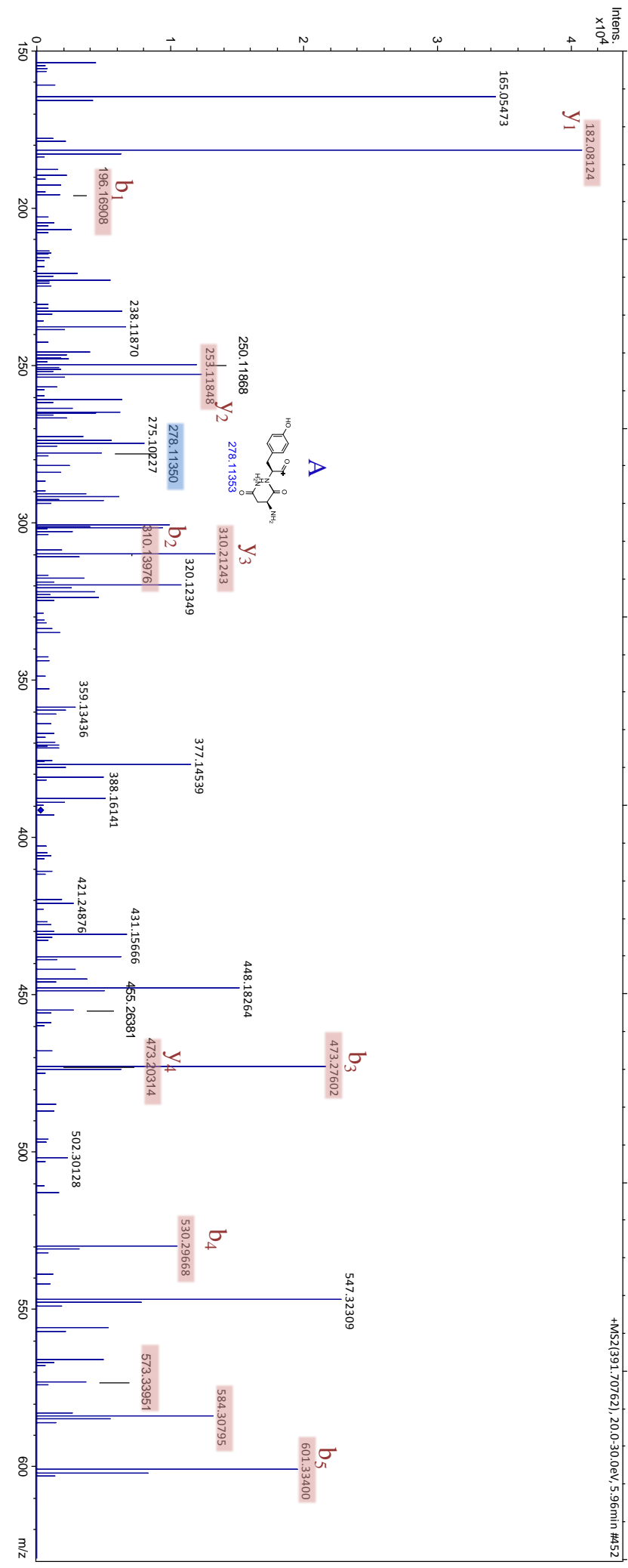

Table S14: Identified $\mathrm{MS}^{2}$ fragments of thaxteramide $\mathrm{C}$

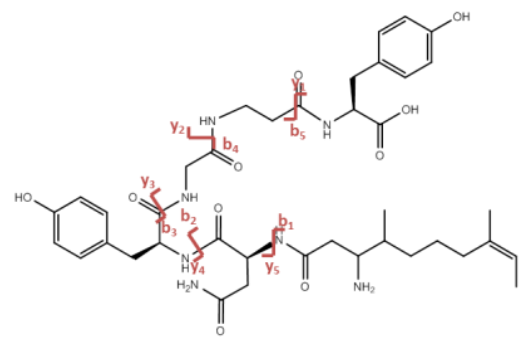

\begin{tabular}{llll}
\hline Ion & Expected & Found & $\begin{array}{l}\Delta \mathbf{m} \\
(\mathbf{p p m})\end{array}$ \\
\hline $\mathbf{H}^{+}$ & 782.40832 & 782.40772 & -0.77 \\
$\mathbf{- H}_{\mathbf{2}} \mathbf{O}$ & 764.39775 & 764.39745 & -0.39 \\
$\mathbf{y}_{\mathbf{4}}$ & 473.20308 & 473.20314 & 0.13 \\
$\mathbf{y}_{\mathbf{3}}$ & 310.13975 & 310.13976 & 0.03 \\
$\mathbf{y}_{\mathbf{2}}$ & 253.11828 & 253.11848 & 0.79 \\
$\mathbf{y}_{\mathbf{1}}$ & 182.08117 & 182.08124 & 0.38 \\
$\mathbf{b}_{\mathbf{5}}$ & 601.33442 & 601.33400 & -0.70 \\
$\mathbf{b}_{\mathbf{5}}-\mathbf{N H} \mathbf{3}$ & 584.30788 & 584.30795 & 0.12 \\
$\mathbf{b}_{\mathbf{5}}-\mathbf{C O}$ & 573.33951 & 573.33951 & 0 \\
$\mathbf{b}_{\mathbf{4}}$ & 530.29731 & 530.29668 & -1.19 \\
$\mathbf{b}_{\mathbf{3}}$ & 473.27585 & 473.27602 & 0.36 \\
$\mathbf{b}_{\mathbf{2}}$ & 310.21252 & 310.21243 & -0.29 \\
$\mathbf{b}_{\mathbf{1}}$ & 196.16959 & 196.16908 & -2.60 \\
$\mathbf{A}$ & 278.11353 & 278.11350 & -0.11 \\
\hline
\end{tabular}

Figure S5: $\mathrm{MS}^{2}$ fragmentation spectrum of thaxteramide $\mathrm{C}$ 


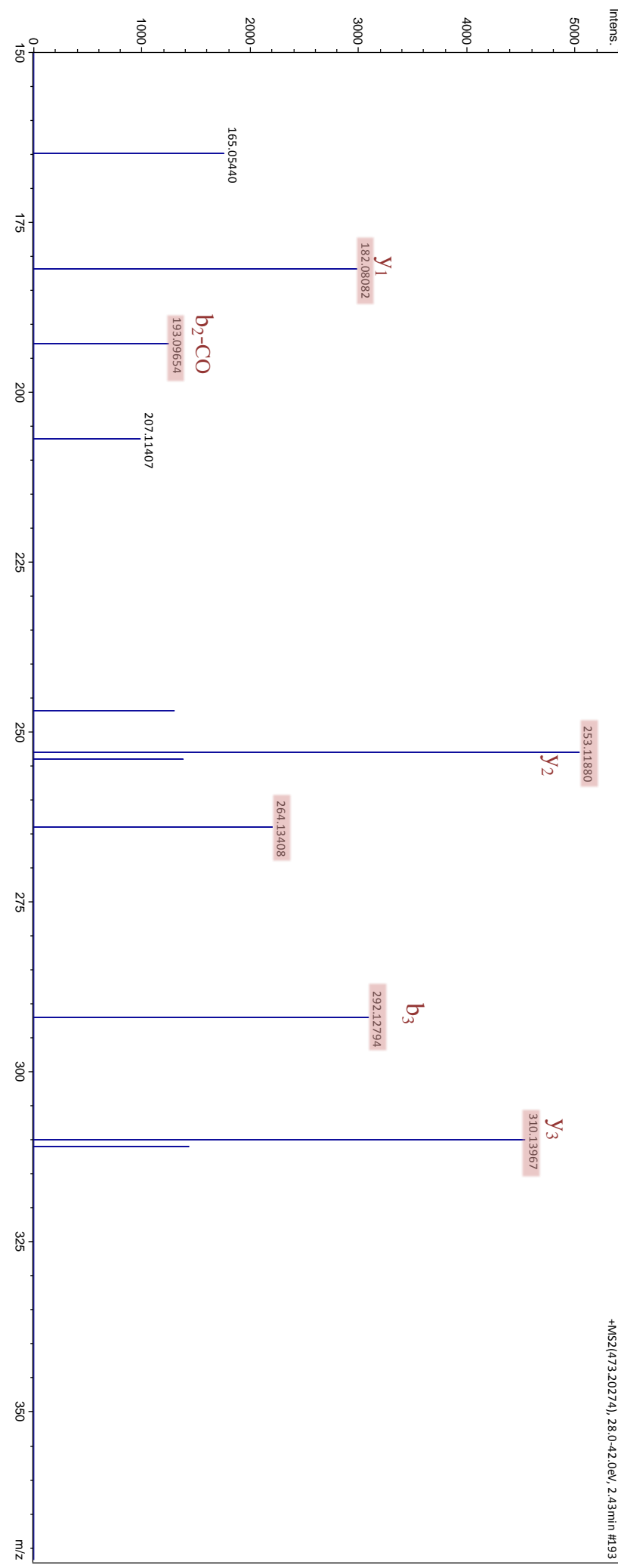

Thaxteramide D

Table S15: Identified $\mathrm{MS}^{2}$ fragments of thaxteramide D

\begin{tabular}{llll}
\hline Ion & Expected & Found & $\mathbf{\Delta m}$ \\
\hline $\mathbf{H}^{+}$ & 473.20308 & 473.20274 & -0.72 \\
$\mathbf{y}_{3}$ & 310.13975 & 310.13967 & -0.26 \\
$\mathbf{y}_{\mathbf{2}}$ & 253.11828 & 253.11880 & 2.05 \\
$\mathbf{y}_{\mathbf{1}}$ & 182.08117 & 182.08082 & -1.92 \\
$\mathbf{b}_{3}$ & 292.12918 & 292.12794 & -4.24 \\
$\mathbf{b}_{\mathbf{3}}$-CO & 264.13427 & 264.13408 & -0.72 \\
$\mathbf{b}_{\mathbf{2}}$-CO & 193.09715 & 193.09654 & -3.16 \\
\hline
\end{tabular}

Figure S6: $\mathrm{MS}^{2}$ fragmentation spectrum of thaxteramide D 


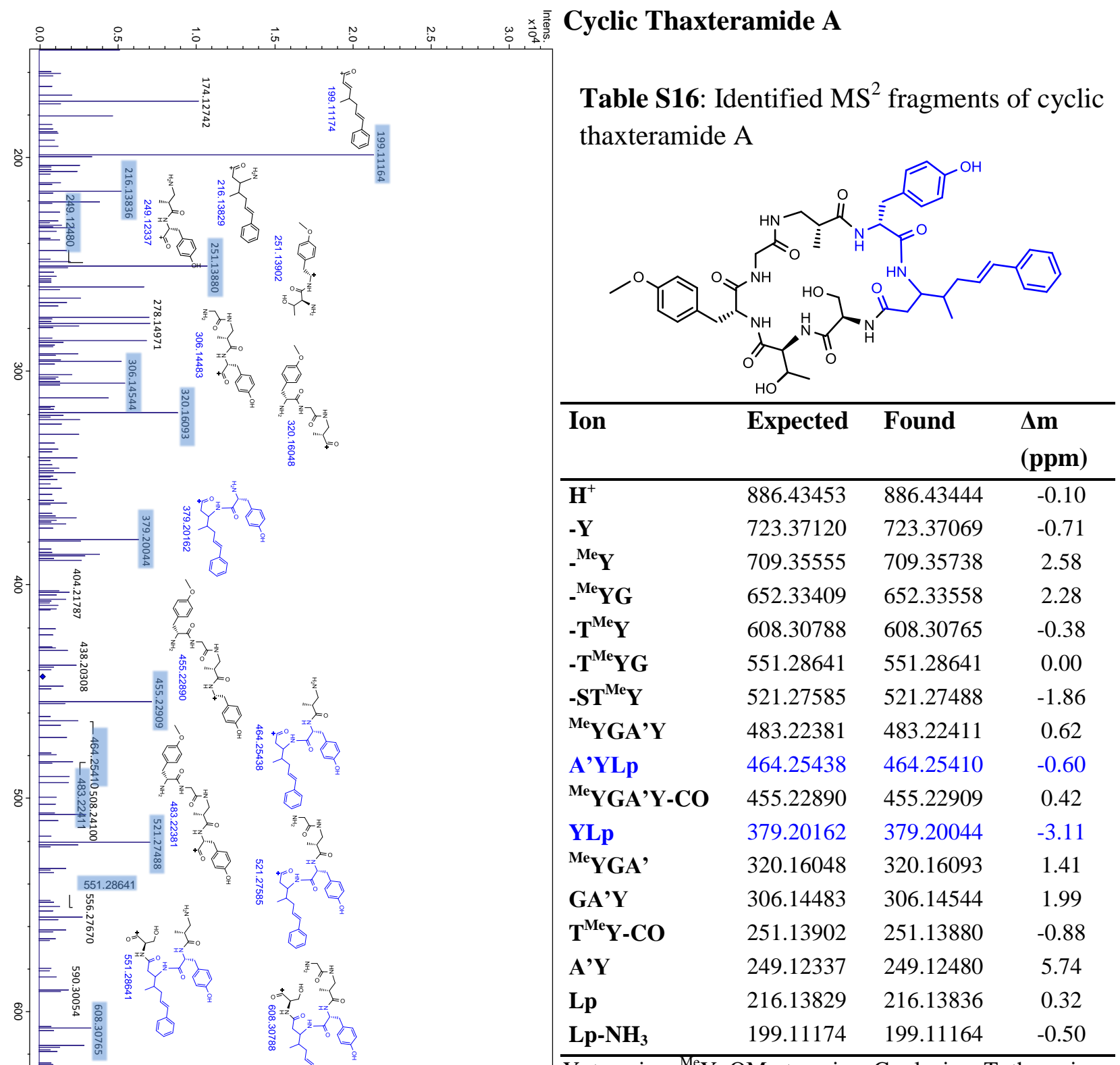

Y: tyrosine; ${ }^{\mathrm{Me}} \mathrm{Y}$ : OMe-tyrosine; G: glycine; T: threonine, $\mathrm{S}$ : serine; $\mathrm{A}^{\prime}$ : Me- $\beta$-alanine; Lp: lipid chain.

Figure S7: $\mathrm{MS}^{2}$ fragmentation spectrum of cyclic thaxteramide A 


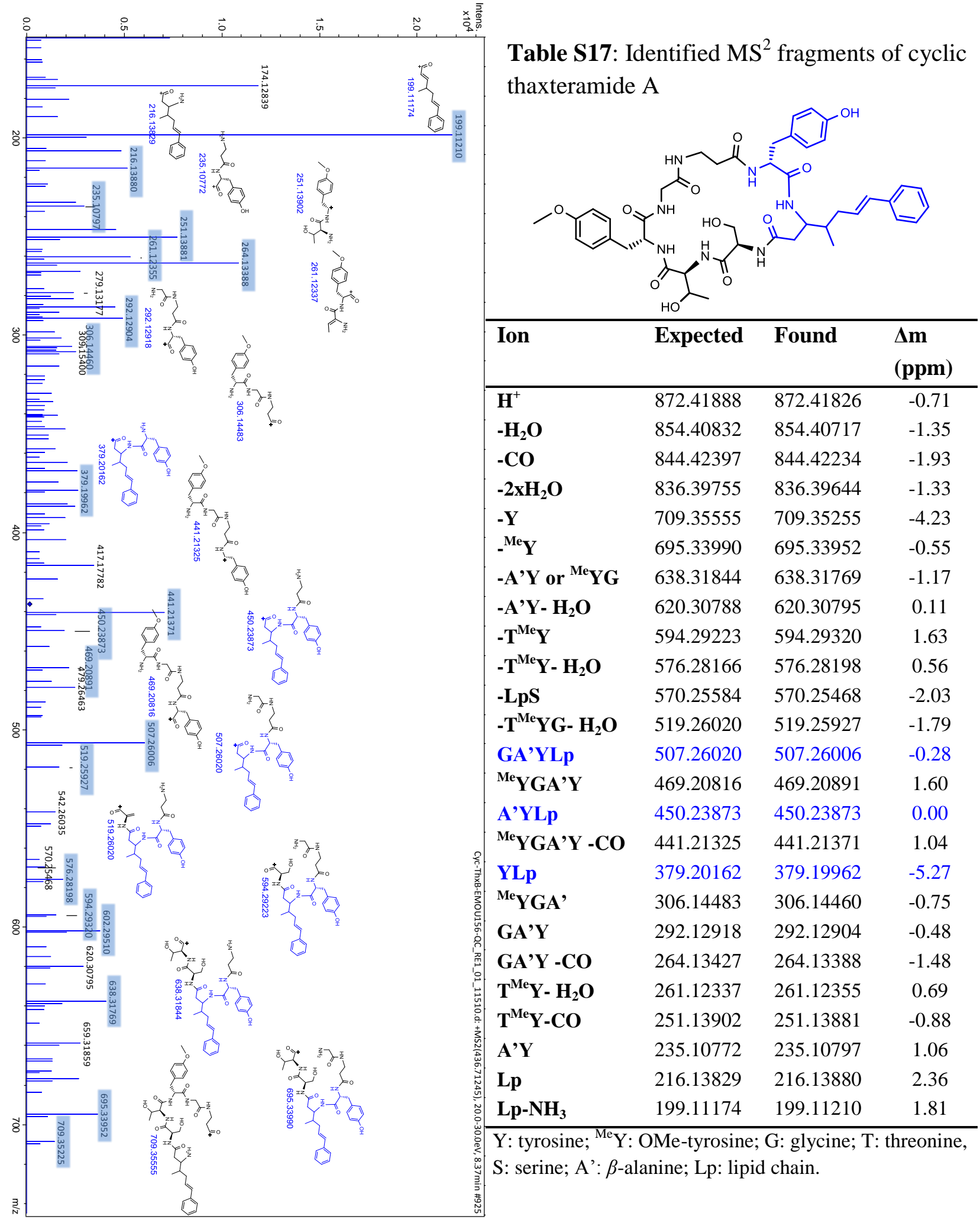

\section{Cyc-Thaxteramide B}

Table S17: Identified $\mathrm{MS}^{2}$ fragments of cyclic xteramide $\mathrm{A}$ 


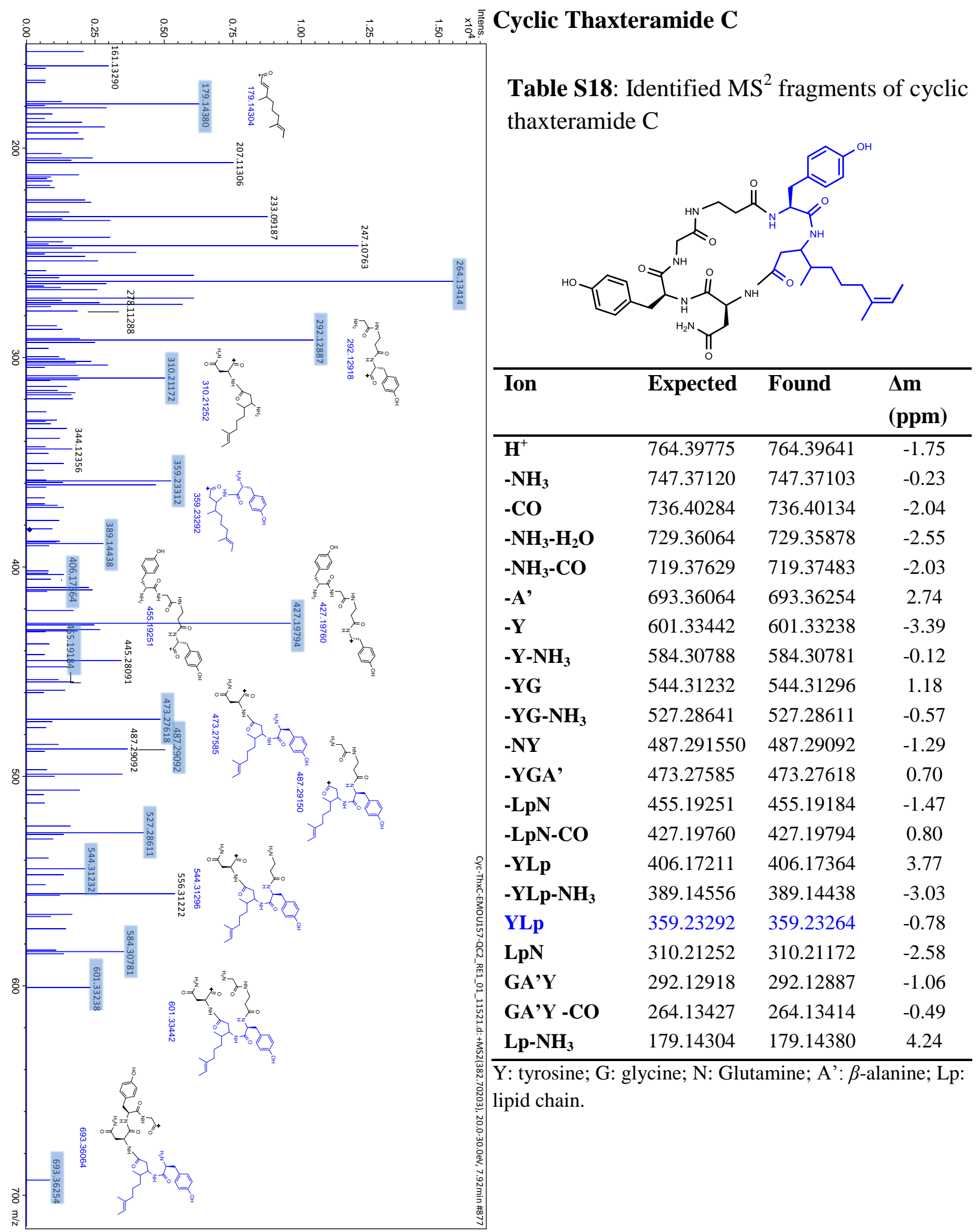

Figure S9: $\mathrm{MS}^{2}$ fragmentation spectrum of cyclic thaxteramide $\mathrm{C}$ 


\section{Marfey's method}

Table S19: Retention times of the D- and L-FDLA derivatized amino acid residues received from the acid hydrolysis of the thaxteramides $\mathrm{A} 1$ and $\mathrm{B} 1$ compared to the respective standards.

\begin{tabular}{|c|c|c|c|c|c|}
\hline \multirow[t]{2}{*}{ Amino acid } & \multicolumn{2}{|c|}{ Standard amino acids } & \multicolumn{2}{|c|}{$\begin{array}{l}\text { Hydrolysate of } \\
\text { Thaxteramide A1 and B1 }\end{array}$} & \multirow[t]{2}{*}{ ThxA1 } \\
\hline & L-FDLA & D-FDLA & L-FDLA & D-FDLA & \\
\hline$L$-Tyrosine & 12.5 & 12.7 & 12.7 & 12.5 & D \\
\hline$L$-Threonine & 12.1 & 15.1 & 121 & 151 & 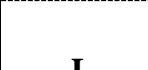 \\
\hline$L$-Allo-Threonine & 12.7 & 13.9 & 12.1 & 15.1 & $\mathbf{L}$ \\
\hline$L$-Serine & 12.3 & 12.8 & 12.3 & 12.8 & $\mathbf{L}$ \\
\hline$O \mathrm{Me}-L$-Tyrosine & 18.0 & 20.3 & 20.3 & 18.0 & D \\
\hline$\beta^{2}$-Homoalanine $(S)$ & 16.2 & 16.1 & 16.1 & 16.2 & $R$ \\
\hline Amino acid & L-FDLA & D-FDLA & L-FDLA & D-FDLA & ThxB1 \\
\hline L-Tyrosine & 12.5 & 12.7 & 12.7 & 12.5 & D \\
\hline L-Threonine & 12.1 & 15.1 & 121 & 151 & $\mathbf{T}$ \\
\hline L-Allo-Threonine & 12.7 & 13.9 & 12.1 & 15.1 & L \\
\hline L-Serine & 12.3 & 12.8 & 12.3 & 12.8 & $\mathbf{L}$ \\
\hline OMe-L-Tyrosine & 18.0 & 20.3 & 20.3 & 18.0 & D \\
\hline
\end{tabular}

Solvent system B was used for HPLC analysis

Table S20: Retention times of the D- and L-FDLA derivatized amino acid residues received from the acid hydrolysis of the thaxteramides $\mathrm{C}$ and $\mathrm{D}$ compared to the respective standards.

\begin{tabular}{l|c|c|c|c|c}
\hline \multirow{2}{*}{ Amino acid } & \multicolumn{2}{|c|}{ Standard amino acids } & \multicolumn{2}{c|}{$\begin{array}{c}\text { Hydrolysate of } \\
\text { Thaxteramide A1 and B1 }\end{array}$} & \multirow{2}{*}{ ThxC } \\
\cline { 2 - 5 } & L-FDLA & D-FDLA & L-FDLA & D-FDLA & \\
\hline$L$-Tyrosine & 12.5 & 12.7 & 12.6 & 12.8 & L \\
\hline$L$-Aspartic acid & 12.5 & 13.3 & 12.6 & 13.4 & L \\
\hline \multicolumn{1}{c|}{ Amino acid } & L-FDLA & D-FDLA & L-FDLA & D-FDLA & ThxD \\
\hline$L$-Tyrosine & 12.5 & 12.7 & 12.5 & 12.7 & L \\
\hline
\end{tabular}

Solvent system B was used for HPLC analysis 


\section{Mosher's method}

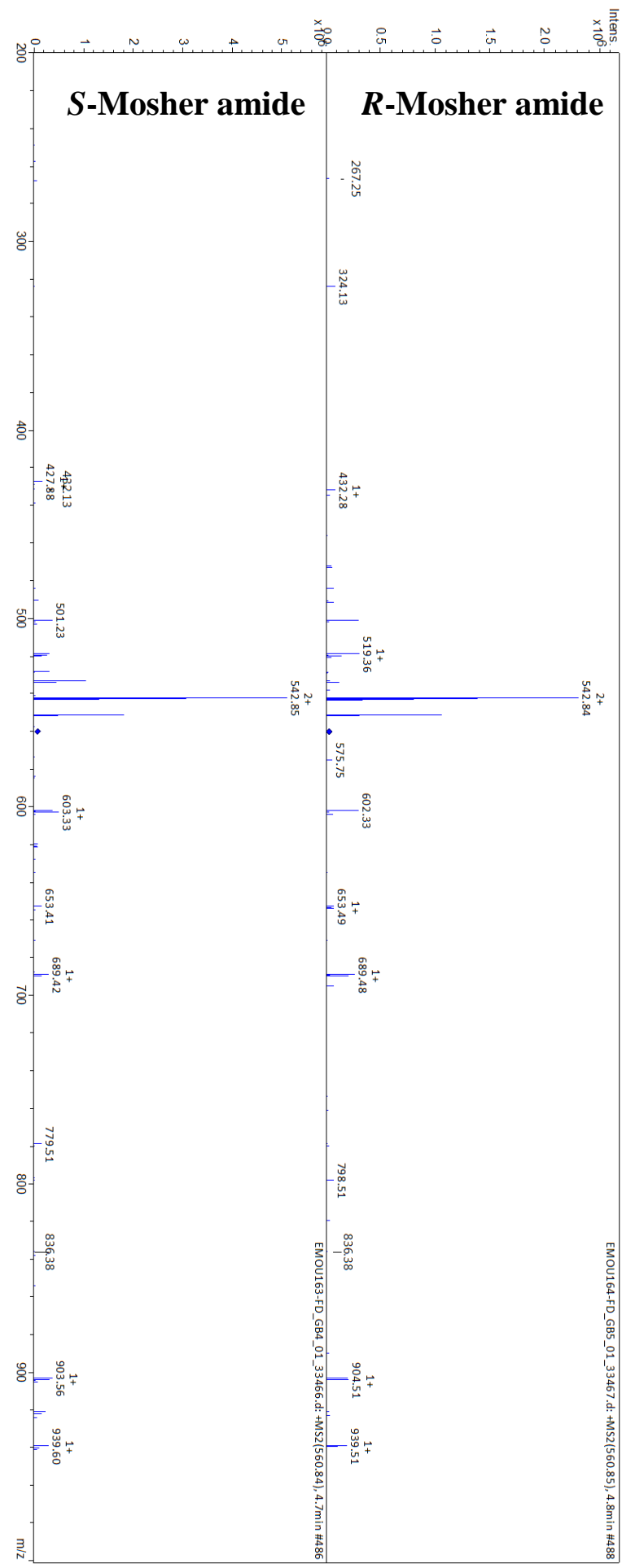

Figure S10: $\mathrm{MS}^{2}$ fragmentation spectra of $(S)$ - and $(R)$-Mosher amide of ThxA1 (major peak at 4.7 and $4.8 \mathrm{~min}$, respectively)

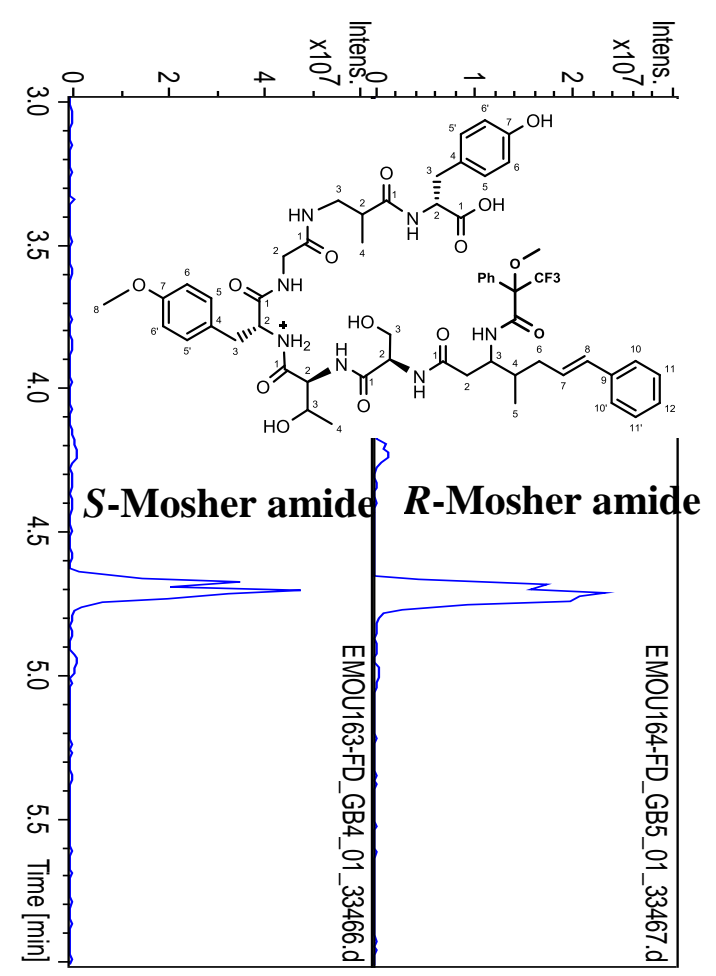

Figure S11: ESI trace at 560.85 of reaction mixtures of $(S)$ - and $(R)$-Mosher amide of ThxA1

Table S21: Identified $\mathrm{MS}^{2}$ fragments of $S$ and $R$-Mosher amide of ThxA1

\begin{tabular}{lll}
\hline Ion & $(\boldsymbol{S})$-amide & $(\boldsymbol{R})$-amide \\
\hline $\mathbf{H}^{+}$ & 1120.71 & 1120.73 \\
$\mathbf{2 H}^{+}$ & 560.84 & 560.85 \\
$\mathbf{2 H}^{+}-\mathbf{H}_{\mathbf{2}} \mathbf{O}$ & 551.80 & 551.82 \\
$\mathbf{2 H}^{+}-\mathbf{2 H}_{\mathbf{2}} \mathbf{O}$ & 542.85 & 542.84 \\
$\mathbf{y}_{\mathbf{6}}$ & 689.42 & 689.48 \\
$\mathbf{y}_{\mathbf{5}}$ & 602.33 & 602.33 \\
$\mathbf{y}_{\mathbf{4}}$ & 501.23 & 501.31 \\
$\mathbf{y}_{\mathbf{3}}$ & - & 324.13 \\
$\mathbf{y}_{\mathbf{1}}$ & - & 267.25 \\
$\mathbf{b}_{\mathbf{6}}$ & 939.60 & 939.51 \\
$\mathbf{b}_{\mathbf{5}}$ & 854.51 & - \\
$\mathbf{b}_{\mathbf{4}}$ & 797.44 & - \\
$\mathbf{b}_{\mathbf{4}}-\mathbf{H}_{\mathbf{2}} \mathbf{O}$ & 836.38 & 836.38 \\
$\mathbf{b}_{\mathbf{3}}-\mathbf{H}_{\mathbf{2}} \mathbf{O}$ & 602.33 & 602.33 \\
$\mathbf{b}_{\mathbf{2}}$ & 519.29 & 519.36 \\
$\mathbf{b}_{\mathbf{1}}$ & 432.28 & 432.13 \\
\hline
\end{tabular}




\section{$(R)$ - and $(S)$ - Mosher amides of thaxteramide A1}

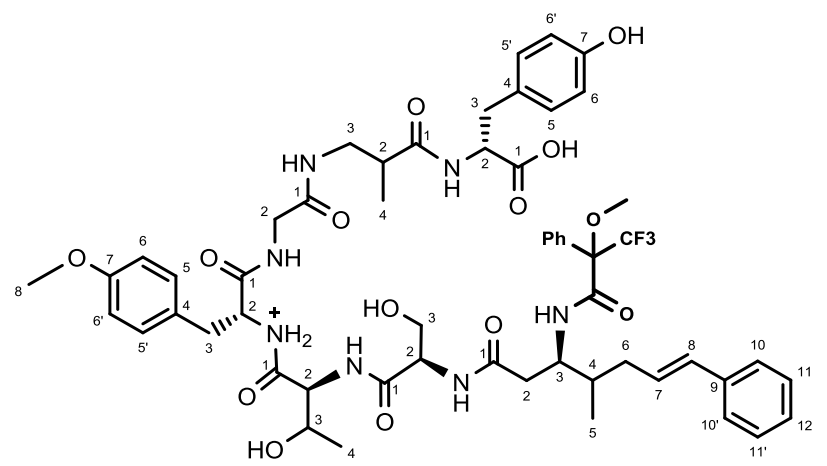

The $(R)$ - and $(S)$-Mosher amide of thaxteramide A1 were synthesised separately as described in procedure $\mathrm{F}$ and analysed without any further purification. The comparison of ${ }^{1} \mathrm{H}$ NMR shifts of the relevant protons of both amides around the C3 stereocenter is detailed in table S22, and indicates an $(R)$-configuration.

Table S22: NMR data of MTPA-derivatives derived from Thaxteramide A1 (in $\mathrm{CD}_{3} \mathrm{OD}$ )

\begin{tabular}{|c|c|c|c|c|c|}
\hline \multirow[b]{2}{*}{$\begin{array}{c}\text { Partial } \\
\text { structure }\end{array}$} & \multirow[b]{2}{*}{$\mathbf{C}$} & \multirow[b]{2}{*}{ Type } & \multirow{2}{*}{$\begin{array}{l}(R) \text {-MTPA amide } \\
\delta_{\mathrm{H}}\left(\delta_{\mathrm{C}}\right)\end{array}$} & \multicolumn{2}{|l|}{ (S)-MTPA amide } \\
\hline & & & & $\boldsymbol{\delta}_{\mathrm{H}}\left(\boldsymbol{\delta}_{\mathrm{C}}\right)$ & $\Delta_{\delta \text { S-R }}$ \\
\hline \multirow{4}{*}{ Serine } & 1 & $\mathrm{CO}$ & & & \\
\hline & 2 & $\mathrm{CH}$ & $4.39(56.2)$ & $4.47(56.5)$ & +0.08 \\
\hline & \multirow[t]{2}{*}{3} & \multirow[t]{2}{*}{$\mathrm{CH}_{2}$} & 3.77 & $3.88(43.3)$ & +0.11 \\
\hline & & & 3.61 & 3.75 & +0.14 \\
\hline & 1 & $\mathrm{CO}$ & & & \\
\hline \multirow[t]{13}{*}{ Lipid chain } & \multirow[t]{2}{*}{2} & \multirow{2}{*}{$\mathrm{CH}_{2}$} & $2.60(37.9)$ & $2.57(37.4)$ & -0.03 \\
\hline & & & 2.64 & 2.63 & -0.01 \\
\hline & 3 & $\mathrm{CH}$ & $4.27(52.5)$ & $4.27(52.0)$ & 0 \\
\hline & 4 & $\mathrm{CH}$ & $1.99(37.9)$ & $1.89(37.6)$ & -0.10 \\
\hline & 5 & $\mathrm{CH}_{3}$ & 1.01 & 0.87 & -0.14 \\
\hline & \multirow[t]{2}{*}{6} & \multirow[t]{2}{*}{$\mathrm{CH}_{2}$} & $2.41(36.9)$ & $2.17(36.7)$ & -0.24 \\
\hline & & & 2.11 & 1.91 & -0.20 \\
\hline & 7 & $\mathrm{CH}$ & 6.27 & $6.16(129.0)$ & -0.11 \\
\hline & 8 & $\mathrm{CH}$ & $6.42(133.0)$ & $6.27(133.0)$ & -0.15 \\
\hline & 9 & $\mathrm{C}$ & & & \\
\hline & $\begin{array}{l}10 \\
10\end{array}$ & $\mathrm{CH}$ & & & \\
\hline & $\begin{array}{l}11, \\
11\end{array}$ & $\mathrm{CH}$ & & & \\
\hline & 12 & $\mathrm{CH}$ & & & \\
\hline
\end{tabular}




\section{Feeding experiments}

Below are the observed mass shifts resulting from precursor incorporations after feeding experiments of the MSr9139 with isotopically labelled precursors strain, as detected by LCMS analysis.

Table S23: Observed mass shifts and their percentages after feeding with isotopically labelled precursors.

\begin{tabular}{|c|c|c|c|c|c|c|}
\hline \multirow[b]{2}{*}{ Precursor } & \multirow[b]{2}{*}{ Exp. } & \multicolumn{5}{|c|}{ Observed mass shift (incorporation \%) } \\
\hline & & $\begin{array}{l}\text { ThxA1 } \\
904.44 \\
\end{array}$ & $\begin{array}{l}\text { ThxA2 } \\
890.42 \\
\end{array}$ & $\begin{array}{l}\text { ThxB1 } \\
890.42 \\
\end{array}$ & $\begin{array}{l}\text { ThxB2 } \\
876.42 \\
\end{array}$ & $\begin{array}{c}\text { ThxC } \\
782.40 \\
\end{array}$ \\
\hline Trans-cinnamic acid- $d_{7}$ & +7 & $\begin{array}{l}911.49 \\
(57 \%) \\
\end{array}$ & $\begin{array}{l}897.48 \\
(62 \%) \\
\end{array}$ & $\begin{array}{l}897.48 \\
(61 \%) \\
\end{array}$ & $\begin{array}{l}883.46 \\
(66 \%) \\
\end{array}$ & - \\
\hline Phenylalanine- $d_{5}$ & +5 & $\begin{array}{l}909.45 \\
(63 \%) \\
\end{array}$ & $\begin{array}{l}895.46 \\
(73 \%)\end{array}$ & $\begin{array}{l}895.47 \\
(69 \%) \\
\end{array}$ & $\begin{array}{l}881.48 \\
(71 \%)\end{array}$ & - \\
\hline Sodium propionate- $1-{ }^{13} \mathrm{C}$ & $+1 /+2$ & $\begin{array}{l}905.45 \\
(74 \%) \\
\end{array}$ & $\begin{array}{l}891.43 \\
(71 \%) \\
\end{array}$ & $\begin{array}{l}891.44 \\
(68 \%) \\
\end{array}$ & $\begin{array}{l}877.42 \\
(62 \%) \\
\end{array}$ & $\begin{array}{l}783.41(25 \%) \\
784.42(53 \%)\end{array}$ \\
\hline Sodium propionate- $2-{ }^{13} \mathrm{C}$ & $+1 /+2$ & $\begin{array}{l}905.45 \\
(66 \%) \\
\end{array}$ & $\begin{array}{l}891.44 \\
(65 \%) \\
\end{array}$ & $\begin{array}{l}891.44 \\
(64 \%) \\
\end{array}$ & $\begin{array}{l}877.42 \\
(64 \%) \\
\end{array}$ & $\begin{array}{l}783.41(29 \%) \\
784.42(33 \%)\end{array}$ \\
\hline Me-Malonic acid- $d_{3}$ & $+3 /+3$ & $\begin{array}{l}907.47 \\
(34 \%)\end{array}$ & $\begin{array}{l}893.45 \\
(32 \%)\end{array}$ & $\begin{array}{l}893.45 \\
(34 \%)\end{array}$ & $\begin{array}{l}879.43 \\
(34 \%)\end{array}$ & $\begin{array}{l}785.43(38 \%) \\
788.45(17 \%)\end{array}$ \\
\hline L-Glutamine- $-{ }^{13} \mathrm{C}_{5},{ }^{15} \mathrm{~N}_{2}$ & +1 & $\begin{array}{l}905.45 \\
(55 \%) \\
\end{array}$ & $\begin{array}{l}891.43 \\
(58 \%) \\
\end{array}$ & $\begin{array}{l}891.43 \\
(58 \%) \\
\end{array}$ & $\begin{array}{l}877.41 \\
(59 \%) \\
\end{array}$ & $783.41(60 \%)$ \\
\hline L-Asparagine- $-{ }^{13} \mathrm{C}_{4},{ }^{15} \mathrm{~N}_{2}$ & $+1 /+6$ & $\begin{array}{l}905.45 \\
(49 \%)\end{array}$ & $\begin{array}{l}891.43 \\
(47 \%)\end{array}$ & $\begin{array}{l}891.43 \\
(51 \%)\end{array}$ & $\begin{array}{l}877.41 \\
(54 \%)\end{array}$ & $\begin{array}{l}783.41(15 \%) \\
788.42(29 \%) \\
789.42(35 \%)\end{array}$ \\
\hline$L$-Glutamic acid- ${ }^{15} \mathrm{~N}$ & +1 & $\begin{array}{l}905.44 \\
(56 \%) \\
\end{array}$ & $\begin{array}{l}891.43 \\
(53 \%) \\
\end{array}$ & $\begin{array}{l}891.43 \\
(52 \%) \\
\end{array}$ & $\begin{array}{l}877.41 \\
(57 \%) \\
\end{array}$ & $783.40(55 \%)$ \\
\hline$L$-Aspartic acid- $-{ }^{13} \mathrm{C}_{4},{ }^{15} \mathrm{~N}$ & $+1 /+5$ & $\begin{array}{l}905.45 \\
(55 \%) \\
\end{array}$ & $\begin{array}{l}891.43 \\
(57 \%)\end{array}$ & $\begin{array}{l}891.43 \\
(54 \%)\end{array}$ & $\begin{array}{l}877.41 \\
(57 \%)\end{array}$ & $783.41(54 \%)$ \\
\hline & & $\begin{array}{l}908.47 \\
(20 \%) \\
\end{array}$ & $\begin{array}{l}894.46 \\
(24 \%)\end{array}$ & $\begin{array}{l}894.46 \\
(22 \%)\end{array}$ & $\begin{array}{l}880.44 \\
(25 \%)\end{array}$ & $\begin{array}{l}786.43 \\
(18 \%)\end{array}$ \\
\hline$L$-Tyrosine-phenyl- $d_{4}$ & $+4 \times 2$ & $\begin{array}{l}912.50 \\
(64 \%) \\
\end{array}$ & $\begin{array}{l}898.48 \\
(63 \%) \\
\end{array}$ & $\begin{array}{l}898.48 \\
(62 \%) \\
\end{array}$ & $\begin{array}{l}884.47 \\
(62 \%) \\
\end{array}$ & $\begin{array}{l}790.46 \\
(74 \%) \\
\end{array}$ \\
\hline$L$-Methionine $-\mathrm{Me}-d_{3}$ & $+3 /+3$ & $\begin{array}{l}907.47 \\
(61 \%) \\
\end{array}$ & - & $\begin{array}{l}893.45 \\
(65 \%) \\
\end{array}$ & - & - \\
\hline Thymine- $d_{4}$ & +4 & $\begin{array}{c}907.46 \\
(36 \%) \\
910.48 \\
54 \%) \\
\end{array}$ & $\begin{array}{l}893.44 \\
(37 \%) \\
896.46 \\
(52 \%) \\
\end{array}$ & $\begin{array}{l}893.44 \\
(12 \%)\end{array}$ & $\begin{array}{l}879.42 \\
(16 \%)\end{array}$ & $\begin{array}{l}785.42(37 \%) \\
788.44(26 \%)\end{array}$ \\
\hline Uracil- $d_{2}$ & +2 & - & - & $\begin{array}{l}891.43 \\
(39 \%) \\
892.44 \\
(34 \%) \\
\end{array}$ & $\begin{array}{l}876.41 \\
(24 \%) \\
877.42 \\
(43 \%) \\
\end{array}$ & $\begin{array}{l}783.41(40 \%) \\
784.42(31 \%)\end{array}$ \\
\hline
\end{tabular}


Tablse S24: $\mathrm{MS}^{2}$ fragmentation of thaxteramides after L-glutamic acid- ${ }^{15} \mathrm{~N}$ feeding

\begin{tabular}{lllllllc}
\hline Ion & $\begin{array}{c}\text { Amino } \\
\text { acid }\end{array}$ & $\begin{array}{c}\text { ThxA1 } \\
904.44\end{array}$ & $\begin{array}{c}\text { ThxA2 } \\
890.42\end{array}$ & $\begin{array}{c}\text { ThxB1 } \\
890.42\end{array}$ & $\begin{array}{c}\text { ThxB2 } \\
876.42\end{array}$ & $\begin{array}{c}\text { Amino } \\
\text { acid }\end{array}$ & $\begin{array}{c}\text { ThxC } \\
782.40\end{array}$ \\
\hline $\mathbf{H}^{+}$ & & 905.44 & 891.43 & 891.43 & 877.42 & & 783.40 \\
$\mathbf{y}_{\mathbf{6}}$ & Lp1 & & & & & - & - \\
$\mathbf{y}_{5}$ & Thr & 602.28 & 588.26 & 588.26 & Lp2 & \\
$\mathbf{y}_{\mathbf{4}}$ & Ser & 501.23 & 487.22 & 487.22 & & Asn & 473.20 \\
$\mathbf{y}_{3}$ & Tyr1 & & 324.15 & 310.14 & Tyr1 & 310.21 \\
$\mathbf{y}_{\mathbf{2}}$ & Gly & & 267.13 & 253.12 & Gly & 253.11 \\
$\mathbf{y}_{\mathbf{1}}$ & $\beta$-Ala & & 182.08 & & & $\beta$-Ala & 182.08 \\
\hline $\mathbf{b}_{\mathbf{6}}$ & Tyr2 & 724.37 & 710.35 & 710.35 & & - & - \\
$\mathbf{b}_{5}$ & $\beta$-Ala & 639.31 & 625.30 & 639.31 & & Tyr2 & 602.33 \\
$\mathbf{b}_{\mathbf{4}}$ & Gly & 582.29 & & 582.29 & & $\beta$-Ala & 531.29 \\
$\mathbf{b}_{3}$ & Tyr1 & & 405.22 & & & Gly & 474.27 \\
$\mathbf{b}_{\mathbf{2}}$ & Ser & 304.16 & & & Tyr1 & \\
$\mathbf{b}_{\mathbf{1}}$ & Thr & & & & Asn & \\
\hline
\end{tabular}

* Isotopically labeled fragments are indicated in red, non-labeled in black.

Tablse S25: $\mathrm{MS}^{2}$ fragmentation of thaxteramides after thymine- $d_{4}$ feeding

\begin{tabular}{|c|c|c|c|c|c|c|c|c|}
\hline \multirow{2}{*}{$\begin{array}{l}\text { Ion } \\
\mathbf{H}^{+}\end{array}$} & \multirow[t]{2}{*}{$\begin{array}{c}\text { Amino } \\
\text { acid }\end{array}$} & \multicolumn{2}{|c|}{$\begin{array}{l}\text { ThxA1 } \\
904.44\end{array}$} & \multicolumn{2}{|c|}{$\begin{array}{l}\text { ThxA2 } \\
890.42\end{array}$} & \multirow[t]{2}{*}{$\begin{array}{c}\text { Amino } \\
\text { acid }\end{array}$} & \multicolumn{2}{|c|}{$\begin{array}{c}\text { ThxCC } \\
782.40\end{array}$} \\
\hline & & 907.46 & 910.48 & 893.44 & 896.46 & & 785.42 & 788.44 \\
\hline $\mathbf{y}_{6}$ & Lp1 & & & & & - & & \\
\hline $\mathbf{y}_{5}$ & Thr & 605.30 & & 591.28 & & Lp2 & & \\
\hline $\mathbf{y}_{4}$ & Ser & 504.25 & & 490.23 & & Asn & 473.20 & \\
\hline $\mathbf{y}_{3}$ & Tyr1 & 327.17 & & 327.17 & & Tyr1 & 310.14 & \\
\hline $\mathbf{y}_{2}$ & Gly & 270.15 & & 270.15 & & Gly & 253.12 & \\
\hline $\mathbf{y}_{1}$ & $\beta$-Ala & 182.08 & & 182.08 & & $\beta$-Ala & & \\
\hline$b_{6}$ & Tyr2 & 726.38 & 729.40 & 712.37 & 715.39 & - & 604.35 & 607.37 \\
\hline$b_{5}$ & $\beta$-Ala & 641.33 & & 627.32 & & Tyr2 & 533.31 & 536.33 \\
\hline $\mathbf{b}_{4}$ & Gly & 584.31 & & 570.30 & & $\beta$-Ala & 476.29 & 479.31 \\
\hline $\mathbf{b}_{3}$ & Tyr1 & & & & & Gly & & \\
\hline $\mathbf{b}_{2}$ & Ser & & & & & Tyr1 & & \\
\hline $\mathbf{b}_{1}$ & Thr & & & & & Asn & & \\
\hline
\end{tabular}

* Doubly labelled fragments are indicated in blue, singly labelled in red, and nonlabelled in black. 


\section{Gene clusters and in silico analyses}

Table S26. Functions of ORFs identified in thxA gene cluster using antiSMASH compared to percentage sequence identity/similarity to previous known proteins by use of BLAST searching

Sequence identity

\begin{tabular}{|c|c|c|c|c|c|c|}
\hline \multirow[b]{2}{*}{ ORF } & \multirow[b]{2}{*}{$\begin{array}{l}\text { Size } \\
\text { [aa] }\end{array}$} & \multirow[b]{2}{*}{ Proposed function } & & & & \\
\hline & & & Closest orthologue & Organism & $\begin{array}{l}\text { SI } \\
{[\%]}\end{array}$ & Reference \\
\hline 1 & 457 & PAS/PAC sensor protein & anti-anti-sigma factor & $\begin{array}{l}\text { Sorangium } \\
\text { cellulosum }\end{array}$ & 63 & KYF51483.1 \\
\hline 2 & 285 & signal peptide protein & Hypothetical protein & $\begin{array}{l}\text { Chondromyces } \\
\text { apiculatus }\end{array}$ & 42 & WP_044237000.1 \\
\hline 3 & 219 & $\begin{array}{l}\text { Imidazole glycerol } \\
\text { phosphate synthase } \\
\text { subunit }\end{array}$ & $\begin{array}{l}\text { Imidazole glycerol } \\
\text { phosphate synthase } \\
\text { subunit HisH }\end{array}$ & $\begin{array}{l}\text { Sorangium } \\
\text { cellulosum }\end{array}$ & 72 & WP_012235455.1 \\
\hline 4 & 195 & HisB protein & HisB protein & $\begin{array}{l}\text { Sorangium } \\
\text { cellulosum }\end{array}$ & 88 & WP_020735415.1 \\
\hline 5 & 2882 & PKS & Polyketide synthase & $\begin{array}{l}\text { Sorangium } \\
\text { cellulosum }\end{array}$ & 57 & CCE88376.1 \\
\hline 6 & 9620 & PKS/NRPS & NRPS & $\begin{array}{l}\text { Nannocystis } \\
\text { exedens }\end{array}$ & 60 & WP_096331950.1 \\
\hline 7 & 292 & Secreted protein & Hypothetical protein & $\begin{array}{l}\text { Sorangium } \\
\text { cellulosum }\end{array}$ & 94 & KYF77099.1 \\
\hline 8 & 806 & Lon protease 2 & DNA-binding protein & $\begin{array}{l}\text { Sorangium } \\
\text { cellulosum }\end{array}$ & 92 & KYF57989.1 \\
\hline 9 & 778 & Hypothetical protein & Hypothetical protein & $\begin{array}{l}\text { Sorangium } \\
\text { cellulosum }\end{array}$ & 70 & KYF63359.1 \\
\hline 10 & 849 & $\begin{array}{l}\text { Two-component hybrid } \\
\text { sensor and regulator }\end{array}$ & $\begin{array}{l}\text { Hybrid sensor } \\
\text { histidine } \\
\text { kinase/response } \\
\text { regulator }\end{array}$ & $\begin{array}{l}\text { Sorangium } \\
\text { cellulosum }\end{array}$ & 74 & KYF56770.1 \\
\hline 11 & 585 & $\begin{array}{l}\text { Multi-sensor hybrid } \\
\text { histidine kinase }\end{array}$ & $\begin{array}{l}\text { Hybrid sensor } \\
\text { histidine } \\
\text { kinase/response } \\
\text { regulator }\end{array}$ & $\begin{array}{l}\text { Sorangium } \\
\text { cellulosum }\end{array}$ & 54 & KYF90994.1 \\
\hline 12 & 261 & $\begin{array}{l}\text { Exodeoxyribonuclease } \\
\text { III }\end{array}$ & $\begin{array}{l}\text { Exodeoxyribonuclease } \\
\text { III }\end{array}$ & $\begin{array}{l}\text { Chondromyces } \\
\text { apiculatus }\end{array}$ & 76 & EYF04658.1 \\
\hline 13 & 568 & Hypothetical protein & Hypothetical protein & $\begin{array}{l}\text { Chondromyces } \\
\text { apiculatus }\end{array}$ & & WP_044243930.1 \\
\hline 14 & 430 & $\begin{array}{l}\text { Histidyl-tRNA } \\
\text { synthetase }\end{array}$ & Histidine--tRNA ligase & $\begin{array}{l}\text { Chondromyces } \\
\text { apiculatus }\end{array}$ & 83 & WP_044243862.1 \\
\hline 15 & 145 & $\begin{array}{l}\text { Holliday junction } \\
\text { resolvase }\end{array}$ & Hypothetical protein & $\begin{array}{l}\text { Sorangium } \\
\text { cellulosum }\end{array}$ & 78 & KYF57648.1 \\
\hline
\end{tabular}

${ }^{\mathrm{a}}$ SI: Sequence identity. Protein Blast (Database: nonredundant protein sequence (nr)). 
Table S27. Functions of ORFs identified in thxA gene cluster using anti-Smash compared to percentage sequence identity/similarity to previous known proteins by use of BLAST searching

\begin{tabular}{|c|c|c|c|c|c|c|}
\hline \multirow[b]{2}{*}{ ORF } & \multirow[b]{2}{*}{$\begin{array}{l}\text { Size } \\
{[\text { aa] }}\end{array}$} & \multirow[b]{2}{*}{ Proposed function } & \multicolumn{4}{|c|}{ Sequence identity } \\
\hline & & & Closest orthologue & Organism & $\begin{array}{c}\text { SI } \\
{[\%]^{\mathrm{a}}}\end{array}$ & Reference \\
\hline 1 & 662 & Hypothetical protein & $\begin{array}{l}\text { Serine/threonine- } \\
\text { protein kinase PknK }\end{array}$ & $\begin{array}{l}\text { Chondromyces } \\
\text { crocatus }\end{array}$ & 67 & WP_050434478 \\
\hline 2 & 267 & $\begin{array}{l}\text { Membrane protein, TerC } \\
\text { family }\end{array}$ & TerC family protein & $\begin{array}{l}\text { Chondromyces } \\
\text { apiculatus }\end{array}$ & 76 & WP_044249890 \\
\hline 3 & 484 & $\begin{array}{l}\text { Methylmalonate- } \\
\text { semialdehyde } \\
\text { dehydrogenase }\end{array}$ & $\begin{array}{l}\text { Methylmalonate- } \\
\text { semialdehyde } \\
\text { dehydrogenase }\end{array}$ & $\begin{array}{l}\text { Chondromyces } \\
\text { apiculatus DSM } \\
436\end{array}$ & 85 & EYF01160 \\
\hline 4 & 290 & $\beta$-ureidopropionase & Acyltransferase & $\begin{array}{l}\text { Chondromyces } \\
\text { apiculatus }\end{array}$ & 91 & WP_044249892 \\
\hline 5 & 467 & Dihydropyrimidinase & Dihydropyrimidinase & $\begin{array}{l}\text { Chondromyces } \\
\text { crocatus }\end{array}$ & 87 & WP_050434483 \\
\hline \multirow{2}{*}{6} & \multirow{2}{*}{460} & \multirow{2}{*}{ Glutamate synthase } & Glutamate synthase & $\begin{array}{l}\text { Chondromyces } \\
\text { crocatus }\end{array}$ & 76 & WP_082363071 \\
\hline & & & $\begin{array}{l}\text { Dihydropyrimidine } \\
\text { dehydrogenase }\end{array}$ & $\begin{array}{l}\text { Chondromyces } \\
\text { crocatus }\end{array}$ & 76 & AKT42929 \\
\hline 7 & 464 & $\begin{array}{l}\text { Dihydropyrimidine } \\
\text { dehydrogenase }\end{array}$ & $\begin{array}{l}\text { Dihydropyrimidine } \\
\text { dehydrogenase }\end{array}$ & $\begin{array}{l}\text { Chondromyces } \\
\text { apiculatus }\end{array}$ & 88 & WP_044249896 \\
\hline 8 & 488 & $\begin{array}{l}\text { Cytosine/purines, uracil, } \\
\text { thiamine, allantoin } \\
\text { transporter }\end{array}$ & Nitrate reductase & $\begin{array}{l}\text { Chondromyces } \\
\text { apiculatus }\end{array}$ & 82 & WP_044249899.1 \\
\hline 9 & 131 & Hypothetical protein & Hypothetical protein & $\begin{array}{l}\text { Anaeromyxobacter } \\
\text { sp. PSR-1 }\end{array}$ & 40 & WP_059436159 \\
\hline 10 & 100 & Hypothetical protein & Pyruvate oxidase & Lactococcus lactis & 52 & WP_096816434 \\
\hline 11 & 194 & $\begin{array}{l}\text { RNA polymerase, } \\
\text { sigma-24 subunit, ECF } \\
\text { subfamily }\end{array}$ & $\begin{array}{l}\text { RNA polymerase } \\
\text { sigma factor }\end{array}$ & $\begin{array}{l}\text { Anaeromyxobacter } \\
\text { sp. PSR-1 }\end{array}$ & 53 & WP_059436157.1 \\
\hline 12 & 264 & $\begin{array}{l}\text { Short-chain } \\
\text { dehydrogenase /reductase } \\
\text { SDR }\end{array}$ & $\begin{array}{l}\text { SDR family } \\
\text { oxidoreductase }\end{array}$ & $\begin{array}{l}\text { Chondromyces } \\
\text { apiculatus }\end{array}$ & 78 & WP_044244231.1 \\
\hline 13 & 723 & $\begin{array}{l}\text { PAS/PAC sensor signal } \\
\text { transduction histidine } \\
\text { kinase }\end{array}$ & $\begin{array}{l}\text { PAS sensor histidine } \\
\text { kinase }\end{array}$ & Cystobacter fuscus & 49 & ATB44042.1 \\
\hline 14 & 476 & Hypothetical protein & Hypothetical protein & $\begin{array}{l}\text { Nannocystis } \\
\text { exedens }\end{array}$ & 40 & SFE91939.1 \\
\hline
\end{tabular}

\footnotetext{
${ }^{a}$ SI: Sequence identity. Protein Blast (Database: nonredundant protein sequence (nr)).
} 
Table S27. Functions of ORFs identified in thxA gene cluster using antiSMASH compared to percentage sequence identity/similarity to previous known proteins by use of BLAST searching (continued)

\begin{tabular}{|c|c|c|c|c|c|c|}
\hline \multirow[b]{2}{*}{ ORF } & \multirow[b]{2}{*}{$\begin{array}{l}\text { Size } \\
\text { [aa] }\end{array}$} & \multirow[b]{2}{*}{ Proposed function } & \multicolumn{4}{|c|}{$\begin{array}{l}\text { Sequence } \\
\text { identity }\end{array}$} \\
\hline & & & $\begin{array}{l}\text { Closest } \\
\text { orthologue }\end{array}$ & Organism & $\begin{array}{c}\text { SI } \\
{[\%]^{\mathrm{a}}}\end{array}$ & Reference \\
\hline 15 & 510 & $\begin{array}{l}\text { Sigma-54 } \\
\text { dependent } \\
\text { transcriptional } \\
\text { regulator }\end{array}$ & $\begin{array}{l}\text { sigma-54- } \\
\text { dependent } \\
\text { transcriptional } \\
\text { regulator }\end{array}$ & $\begin{array}{l}\text { Chondromyces } \\
\text { apiculatus }\end{array}$ & 64 & $\begin{array}{l}\text { WP_044249902 } \\
.1\end{array}$ \\
\hline 16 & 239 & $\begin{array}{l}\text { Hypothetical } \\
\text { protein }\end{array}$ & $\begin{array}{l}\text { Hypothetical } \\
\text { protein }\end{array}$ & $\begin{array}{l}\text { Chondromyces } \\
\text { apiculatus }\end{array}$ & 39 & $\begin{array}{l}\text { WP_044249904 } \\
.1\end{array}$ \\
\hline 17 & 6331 & PKS & PKS type I & $\begin{array}{l}\text { Sorangium } \\
\text { cellulosum }\end{array}$ & 56 & $\begin{array}{l}\text { WP_104980970 } \\
.1\end{array}$ \\
\hline 18 & 7250 & PKS/NRPS & NRPS & $\begin{array}{l}\text { Archangium } \\
\text { gephyra }\end{array}$ & 44 & $\begin{array}{l}\text { WP_047857561 } \\
.1\end{array}$ \\
\hline 19 & 1717 & Protein kinase & $\begin{array}{l}\text { Hypothetical } \\
\text { protein }\end{array}$ & $\begin{array}{l}\text { Sorangium } \\
\text { cellulosum }\end{array}$ & 61 & KYF99469.1 \\
\hline 20 & 428 & $\begin{array}{l}\text { Major facilitator } \\
\text { superfamily }\end{array}$ & MFS transporter & $\begin{array}{l}\text { Chondromyces } \\
\text { apiculatus }\end{array}$ & 38 & $\begin{array}{l}\text { WP_081865306 } \\
.1\end{array}$ \\
\hline 21 & 516 & $\begin{array}{l}\text { Hypothetical } \\
\text { protein }\end{array}$ & $\begin{array}{l}\text { Hypothetical } \\
\text { protein }\end{array}$ & $\begin{array}{l}\text { Sorangium } \\
\text { cellulosum }\end{array}$ & 61 & KYF76308.1 \\
\hline 22 & 367 & $\begin{array}{l}\text { Membrane protease } \\
\text { subunit } \\
\text { stomatin/prohibitin- } \\
\text { like protein }\end{array}$ & $\begin{array}{l}\text { Hypothetical } \\
\text { protein }\end{array}$ & $\begin{array}{l}\text { Sorangium } \\
\text { cellulosum }\end{array}$ & 76 & KYF76307.1 \\
\hline 23 & 226 & $\begin{array}{l}\text { Hypothetical } \\
\text { protein }\end{array}$ & $\begin{array}{l}\text { DUF697 domain- } \\
\text { containing protein }\end{array}$ & $\begin{array}{l}\text { Sorangium } \\
\text { cellulosum }\end{array}$ & 74 & $\begin{array}{l}\text { WP_061611893 } \\
.1\end{array}$ \\
\hline 24 & 557 & $\begin{array}{l}\text { ABC-type } \\
\text { siderophore export } \\
\text { system, fused } \\
\text { ATPase and } \\
\text { permease } \\
\text { components }\end{array}$ & $\begin{array}{l}\text { cyclic peptide } \\
\text { export ABC } \\
\text { transporter }\end{array}$ & $\begin{array}{l}\text { Sorangium } \\
\text { cellulosum }\end{array}$ & 71 & $\begin{array}{l}\text { WP_081426940 } \\
.1\end{array}$ \\
\hline 25 & 444 & $\begin{array}{l}\text { Hypothetical } \\
\text { protein }\end{array}$ & $\begin{array}{l}\text { Hypothetical } \\
\text { protein }\end{array}$ & $\begin{array}{l}\text { Sorangium } \\
\text { cellulosum }\end{array}$ & 51 & KYF84429.1 \\
\hline 26 & 978 & Protein kinase & $\begin{array}{l}\text { serine/threonine- } \\
\text { protein kinase } \\
\text { PknK }\end{array}$ & $\begin{array}{l}\text { Chondromyces } \\
\text { crocatus }\end{array}$ & 67 & $\begin{array}{l}\text { WP_050434478 } \\
.1\end{array}$ \\
\hline
\end{tabular}

${ }^{a}$ SI: Sequence identity. Protein Blast (Database: nonredundant protein sequence (nr)). 


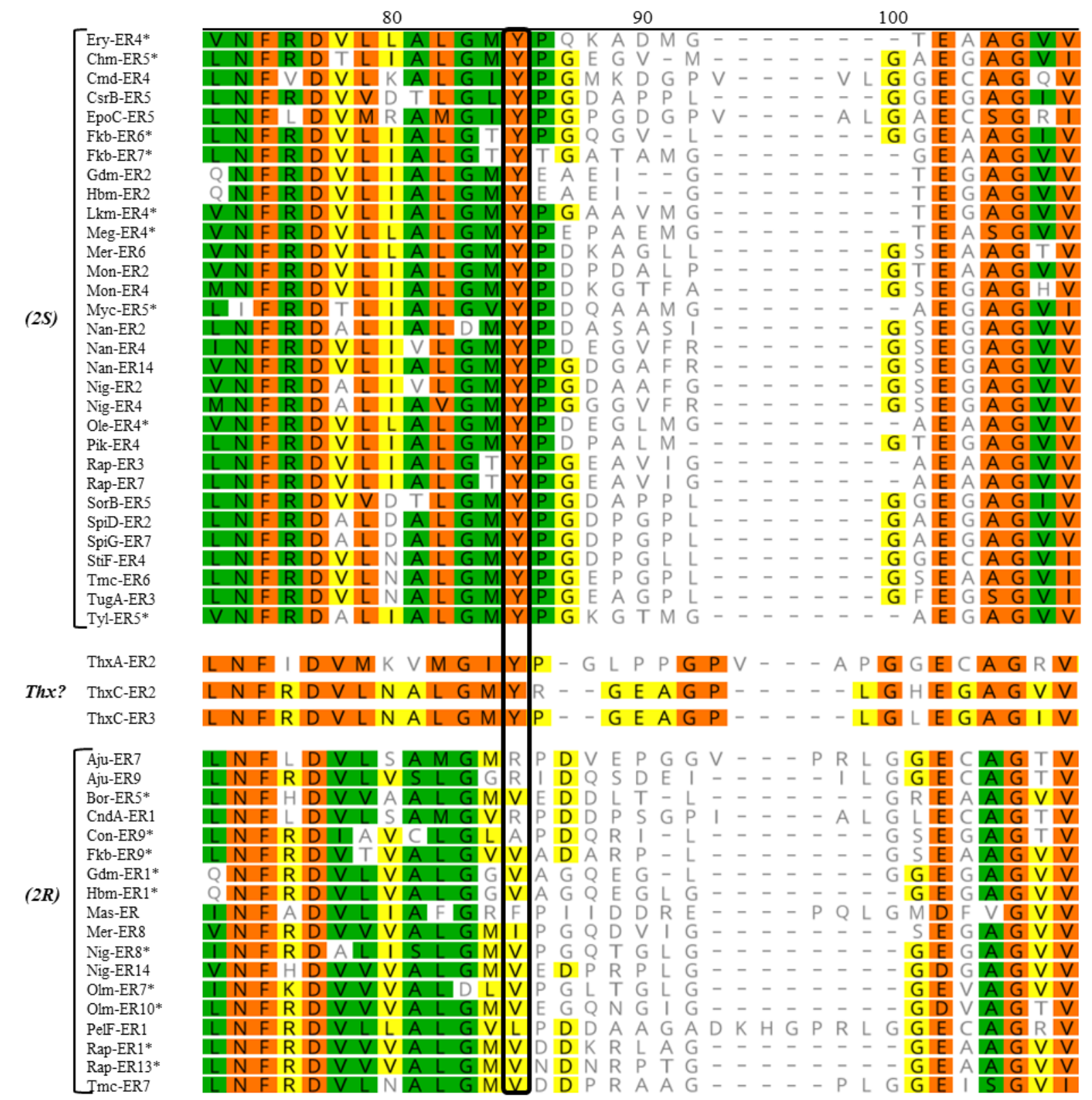

Figure S12: Sequence alignment of a portion of known ER domains from PKS genes based on the stereochemical outcome of the methyl group $((R)$ or $(S)$-configuration) at the $\mathrm{C} 2$ position resulting from the enzymatic reduction of the carbon double bond of the 2-enoyl intermediate, compared to the ER domains in module 2 of thxA and modules 2 and 3 of thxC. The highlighted alignment depicts the presence of the conserved tyrosine (Y85) residue previously correlated with $(2 S)$ configuration in the polyketide products in all ER domains of thxA and thxC. Residue numbering is that of the longest ER domain (CsrB-ER5) in the alignement. *ER domains labeled with an asterix were used in the initial study by Leadlay et al. 


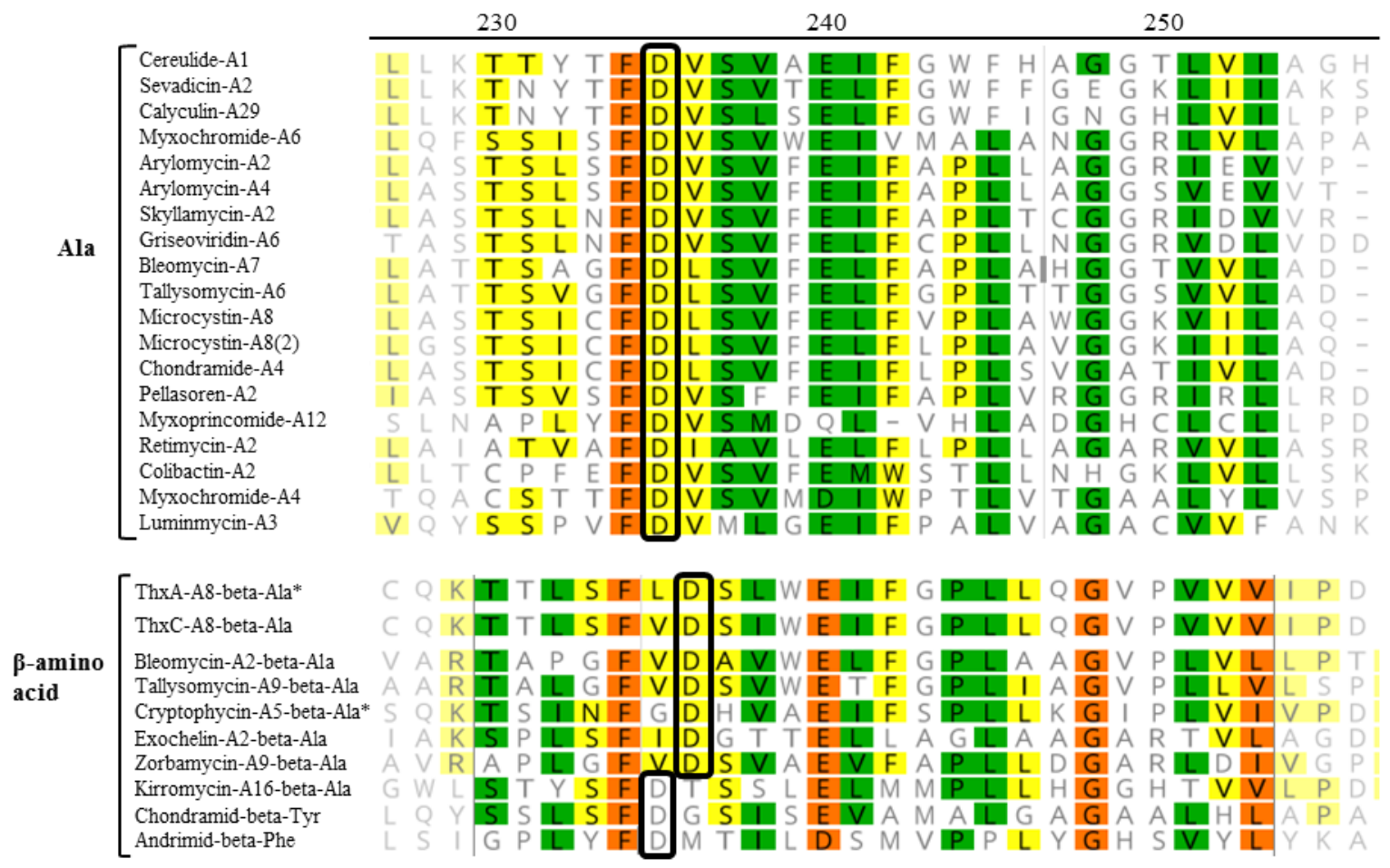

Figure S13: Sequence alignment of a portion of known A domains from PKS genes, of which the specificity has been determined to be for alanine or other $\beta$-amino acids, compared to the A domains in thxA and thxC with $\beta$-amino acid specificity. The highlighted alignment depicts the presence of the conserved aspartic acid (D235) residue within the active site of A domains for alanine specificity, and showing a one position shift for most $\beta$-alanine specificities including ThxA-A8 and ThxC-A8. Those of kirromycin ( $\beta$-Ala), chondramid $(\beta$-Tyr), and andrimid $(\beta$ Phe) retain the same position. Residue numbering is based on Gramicidin PheA domain (not shown). *A domains proven to accommodate both $\beta$-Ala and Me- $\beta$-Ala. 
Table S28: Active site residues of known A domains from PKS genes, of which the specificity has been determined to be for alanine or other $\beta$-amino acids, compared to the A domains in modules 8 of thxA and thx $C$ with $\beta$-amino acid specificity.

\begin{tabular}{|c|c|c|c|c|c|c|c|c|c|c|}
\hline & Selectivity & 235 & 236 & 239 & 278 & 299 & 301 & 322 & 330 & 331 \\
\hline Consensus & Ala & D & $\mathbf{L}$ & $\mathbf{L}$ & $\mathbf{F}$ & $\mathbf{G}$ & $\mathbf{I}$ & $\mathbf{A}$ & $\mathbf{V}$ & $\mathbf{L}$ \\
\hline Cereulide-A1 & Ala & D & $\mathrm{V}$ & A & $\mathrm{N}$ & I & $\mathrm{A}$ & $\mathrm{L}$ & I & $\mathrm{Y}$ \\
\hline Sevadicin-A2 & Ala & D & $\mathrm{V}$ & $\mathrm{T}$ & $\mathrm{N}$ & $\mathrm{F}$ & $\mathrm{A}$ & $\mathrm{L}$ & $\mathrm{I}$ & $\mathrm{Y}$ \\
\hline Calyculin-A29 & Ala & D & $\mathrm{V}$ & $\mathrm{S}$ & $\mathrm{N}$ & $\mathrm{M}$ & $\mathrm{A}$ & I & I & $\mathrm{Y}$ \\
\hline Myxochromide-A6 & Ala & D & $\mathrm{V}$ & $\mathrm{W}$ & $\mathrm{V}$ & $\mathrm{L}$ & $\mathrm{A}$ & $\mathrm{A}$ & I & I \\
\hline Arylomycin-A2 & Ala & D & $\mathrm{V}$ & $\mathrm{F}$ & $\mathrm{S}$ & $\mathrm{V}$ & $\bar{A}$ & $\mathrm{~L}$ & $\mathrm{~V}$ & $\mathrm{Y}$ \\
\hline Arylomycin-A4 & Ala & D & $\mathrm{V}$ & $\mathrm{F}$ & $S$ & $\mathrm{~V}$ & $\mathrm{~A}$ & $\mathrm{~L}$ & V & $\mathrm{Y}$ \\
\hline Skyllamycin-A2 & Ala & D & $\mathrm{V}$ & $\mathrm{F}$ & $S$ & $\mathrm{~V}$ & $\mathrm{~A}$ & $\mathrm{~F}$ & $\mathrm{~V}$ & $\mathrm{Y}$ \\
\hline Griseoviridin-A6 & Ala & D & $\mathrm{V}$ & $\mathrm{F}$ & $S$ & $\mathrm{~L}$ & $\mathrm{~A}$ & $\mathrm{~V}$ & $\mathrm{~V}$ & $\mathrm{Y}$ \\
\hline Bleomycin-A7 & Ala & D & $\mathrm{L}$ & $\mathrm{F}$ & $\mathrm{N}$ & $\mathrm{N}$ & A & $\mathrm{L}$ & $\mathrm{T}$ & $\mathrm{Y}$ \\
\hline Tallysomycin-A6 & Ala & D & $\mathrm{L}$ & 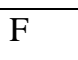 & $\mathrm{N}$ & $\mathrm{N}$ & $\mathrm{A}$ & $\mathrm{L}$ & $\mathrm{T}$ & $\mathrm{Y}$ \\
\hline Microcystin-A8 & Ala & D & $\mathrm{L}$ & $\mathrm{F}$ & $\mathrm{N}$ & $\mathrm{N}$ & A & $\mathrm{L}$ & $\mathrm{T}$ & $\mathrm{Y}$ \\
\hline Microcystin-A8(2) & Ala & D & $\mathrm{L}$ & $\mathrm{F}$ & $\mathrm{N}$ & $\mathrm{N}$ & $\mathrm{A}$ & $\mathrm{L}$ & $\mathrm{T}$ & $\mathrm{Y}$ \\
\hline Chondramide-A4 & Ala & D & $\mathrm{L}$ & $\mathrm{F}$ & $\mathrm{N}$ & $\mathrm{N}$ & $\mathrm{A}$ & $\mathrm{L}$ & $\mathrm{T}$ & $\mathrm{Y}$ \\
\hline Pellasoren-A2 & Ala & D & $\mathrm{V}$ & $\bar{F}$ & $\mathrm{~V}$ & $\mathrm{~N}$ & $\mathrm{~A}$ & $\mathrm{~L}$ & $\mathrm{~T}$ & $\mathrm{Y}$ \\
\hline Myxoprincomide-A12 & Ala & D & V & M & $\mathrm{D}$ & $\mathrm{L}$ & A & A & V & $\mathrm{Y}$ \\
\hline Retimycin-A2 & Ala & D & I & $\mathrm{L}$ & $\mathrm{Q}$ & I & $\mathrm{T}$ & $\mathrm{L}$ & $\mathrm{V}$ & $\mathrm{Y}$ \\
\hline Colibactin-A2 & Ala & D & $\mathrm{V}$ & $\mathrm{F}$ & $\mathrm{W}$ & $\mathrm{T}$ & $\mathrm{G}$ & $\mathrm{G}$ & I & $\mathrm{F}$ \\
\hline Myxochromide-A4 & Ala & D & $\mathrm{V}$ & $\mathrm{M}$ & $\mathrm{S}$ & I & $\mathrm{G}$ & I & $\mathrm{V}$ & A \\
\hline Luminmycin-A3 & Ala & $\mathrm{D}$ & $\mathrm{V}$ & G & $\mathrm{W}$ & I & $\mathrm{T}$ & G & I & V \\
\hline ThxA-A8- $\beta$-Ala* & $\beta$-Ala & $\mathrm{L}$ & $\mathrm{D}$ & $\mathrm{W}$ & $\mathrm{V}$ & $\mathrm{T}$ & $\mathrm{S}$ & $\mathrm{L}$ & A & $\mathrm{D}$ \\
\hline ThxC-A8- $\beta$-Ala & $\beta$-Ala & $\mathrm{V}$ & D & $\mathrm{W}$ & V & $\mathrm{T}$ & $\mathrm{S}$ & $\mathrm{L}$ & A & $\mathrm{D}$ \\
\hline Bleomycin-A2- $\beta$-Ala & $\beta$-Ala & $\overline{\mathrm{V}}$ & $\bar{D}$ & $\overline{\mathrm{W}}$ & $\overline{\mathrm{V}}$ & $\bar{I}$ & $\bar{S}$ & $\mathrm{~L}$ & $\mathrm{~A}$ & $\mathrm{D}$ \\
\hline Tallysomycin-A9- $\beta$-Ala & $\beta$-Ala & $\mathrm{V}$ & D & $\mathrm{W}$ & $\mathrm{V}$ & $\mathrm{V}$ & $\mathrm{S}$ & $\mathrm{L}$ & A & $\mathrm{D}$ \\
\hline Cryptophycin-A5- $\beta$-Ala* & $\beta$-Ala & G & D & A & $\mathrm{V}$ & $\mathrm{F}$ & $\mathrm{S}$ & $\mathrm{L}$ & A & $\mathrm{D}$ \\
\hline Exochelin-A2- $\beta$-Ala & $\beta$-Ala & I & D & $\mathrm{T}$ & $\mathrm{L}$ & I & $\mathrm{S}$ & $S$ & G & $\mathrm{D}$ \\
\hline Zorbamycin-A9- $\beta$-Ala & $\beta$-Ala & $\mathrm{V}$ & $\mathrm{D}$ & A & $\mathrm{L}$ & $\mathrm{V}$ & $\mathrm{S}$ & $\mathrm{L}$ & A & $\mathrm{D}$ \\
\hline Kirromycin-A16- $\beta$-Ala & $\beta$-Ala & D & $\mathrm{T}$ & $\mathrm{L}$ & Q & $\mathrm{L}$ & G & V & I & $\mathrm{W}$ \\
\hline Chondramid- $\beta$-Tyr & $\beta$-Tyr & $\mathrm{D}$ & $\mathrm{G}$ & $S$ & $\mathrm{~T}$ & $\mathrm{I}$ & $\mathrm{T}$ & $\bar{A}$ & $\mathrm{~V}$ & $\bar{A}$ \\
\hline Andrimid- $\beta$-Phe & $\beta$-Phe & $\mathbf{D}$ & $\mathrm{M}$ & $\mathrm{L}$ & $S$ & $\mathrm{~L}$ & $\mathrm{G}$ & A & $\mathrm{M}$ & $\mathrm{C}$ \\
\hline
\end{tabular}

* A domains proven to accommodate both $\beta$-Ala and Me- $\beta$-Ala. 


\section{Epimerization}

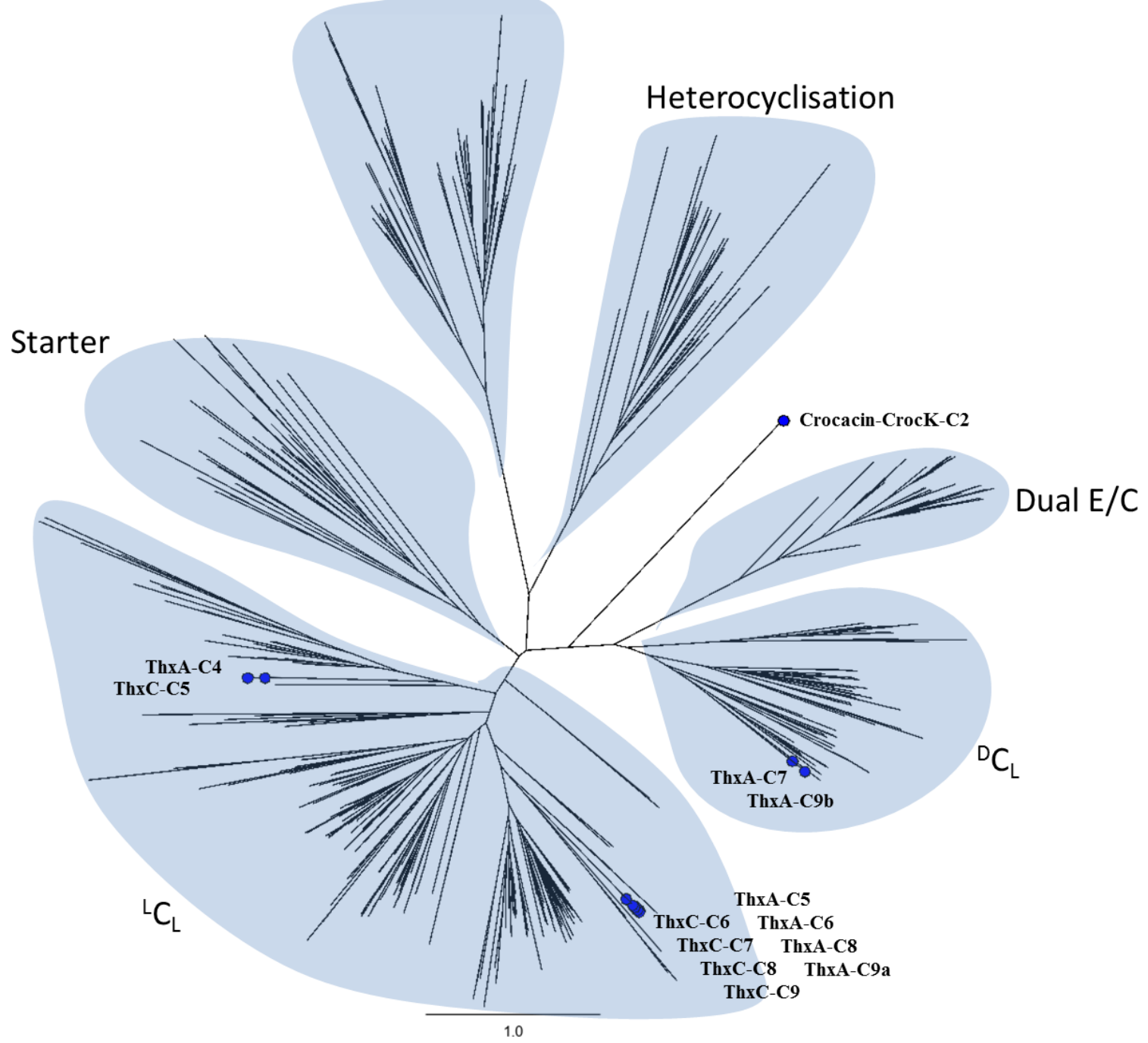

Figure S14: Phylogenetic tree of condensation domains (C) including those involved in the biosynthesis of thaxteramides as well as the known terminal $\mathrm{C}$ domain CrocK-C2 from the crocacin biosynthetic pathway.

\section{MT domain in module 6 of thxA:}

ARRRRAGSRRIRHRAVPGRAVAVGRGVLRVRRRPLWLDGGARGAQPRLPRRVRAEPRGQDGRGDRPGAP RDPLPDVRRGGGPAGLRRRVPERVVRKGAGDRAFARPRGPDHRRPRRRAGGRAPGAGGLVRVGDRRRDR RLRGRGAAREQRAAAAPEPRGDDPLAERHPHRRREPAPGLSVFILSWRRPLRRAHLRGRGRAVRSPRLHEA PLARGPALHRRHLRRPRLHRRCPGRGRARDHALVHVRRGLPRVPGLADAVHRPGSLP*RARLAGELAARL HPRLGGGHRRIGGRSAGGHDRAYALRQRAQP*FRDPGDTRAPGSGAAEHRLPVSSSGSRLPGQPVLRAAVP PRRGACGRAAGRRGAARAPRRAAAAVHDARRVRDDAVVPDDHGWQGGPQGAAAARGGAREREGPRGA SER

The in silico structural homology prediction of the MT domain of module 6 indicates a homology to histone-arginine methyltransferase where 329 residues ( $84 \%$ of the sequence) have been modelled with $100.0 \%$ confidence by the single highest scoring template. 


\section{Bioactivity data}

The observed minimum inhibitory concentration (MIC, $\mu \mathrm{g} / \mathrm{mL}$ ) values of active derivatives are shown below in table S29. If not included in the table, the MIC of any of the derivatives against any of the pathogens could not be determined, as it would be above the maximum concentration of $64 \mu \mathrm{g} / \mathrm{mL}$ of our experimental procedure. In this case, the derivative is reported as showing no activity.

Table S29: MIC values of active derivatives against the corresponding pathogens

$\mathrm{MIC}[\mu \mathrm{g} / \mathrm{mL}]$

\begin{tabular}{lcc}
\cline { 2 - 3 } Pathogen & Cyc-ThxA & Cyc-ThxC \\
\hline Staphylococcus aureus Newman & 8 & 32 \\
Bacillus subtilis & 4 & 16 \\
S. pneumoniae DSM-20566 & 16 & 16 \\
S. aureus N315 (MRSA) & $>64$ & 32 \\
S. aureus HG001 & 64 & 32 \\
S. aureus HG001 Dap (DRSA) & $>64$ & 64 \\
\hline
\end{tabular}




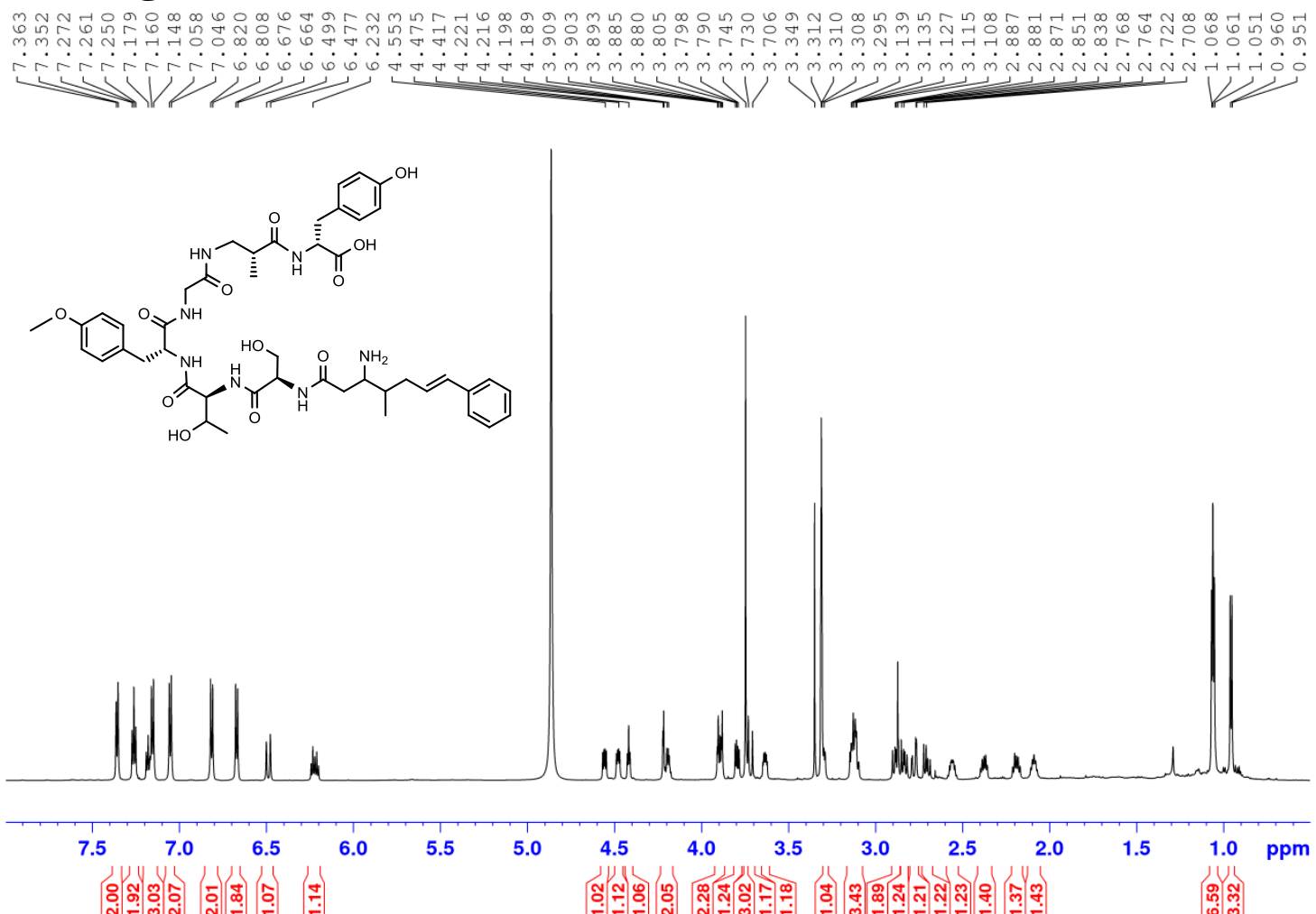

Figure S15: ${ }^{1} \mathrm{H}$ spectrum of Thaxteramide $\mathrm{A} 1\left(\mathrm{CD}_{3} \mathrm{OD}, 700 \mathrm{MHz}\right)$

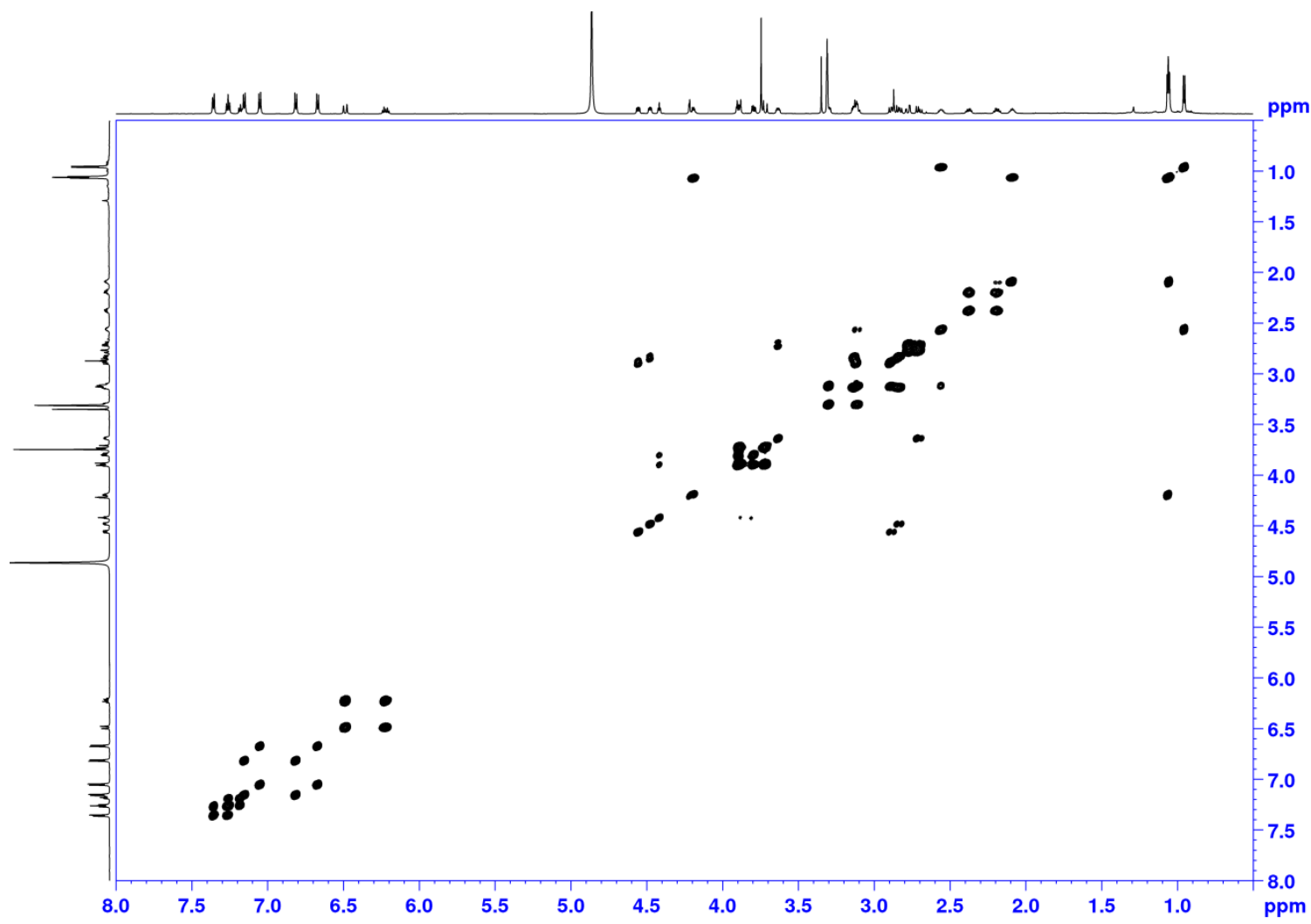

Figure S16: COSY spectrum of Thaxteramide $\mathrm{A} 1\left(\mathrm{CD}_{3} \mathrm{OD}, 700 \mathrm{MHz}\right)$ 


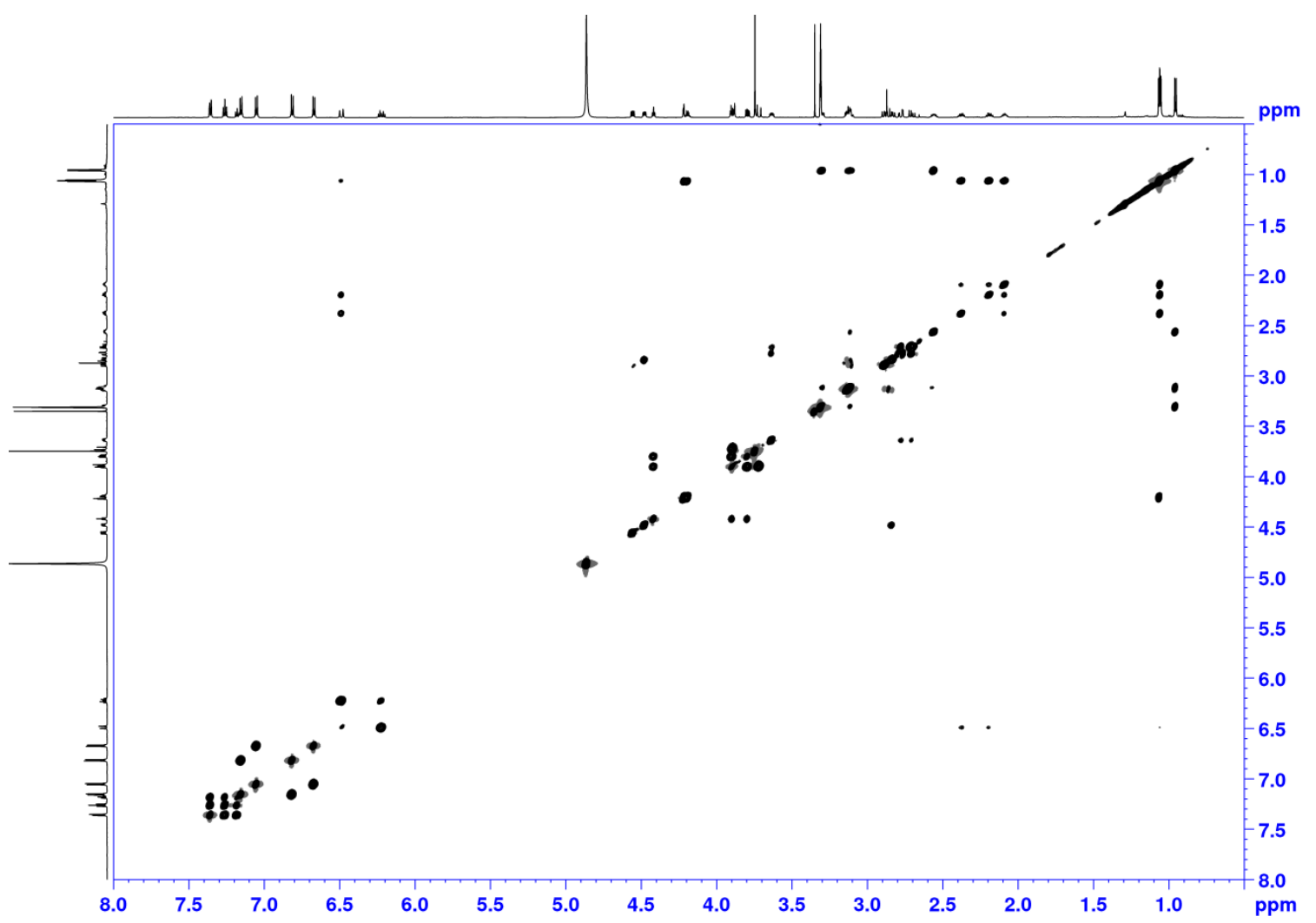

Figure S17: TOCSY spectrum of Thaxteramide A1 $\left(\mathrm{CD}_{3} \mathrm{OD}, 700 \mathrm{MHz}\right)$

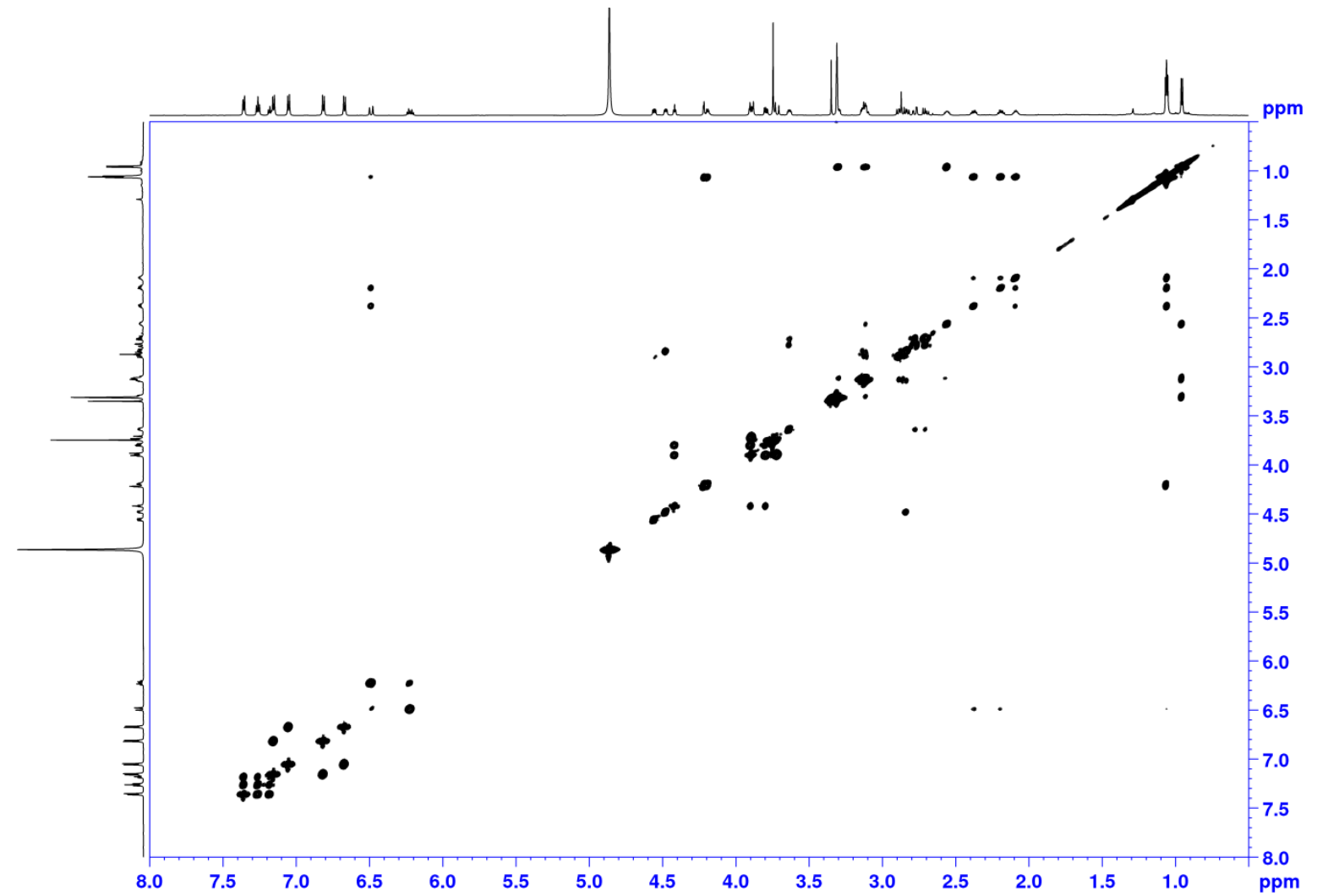

Figure S18: ROESY spectrum of Thaxteramide A1 $\left(\mathrm{CD}_{3} \mathrm{OD}, 700 \mathrm{MHz}\right)$ 


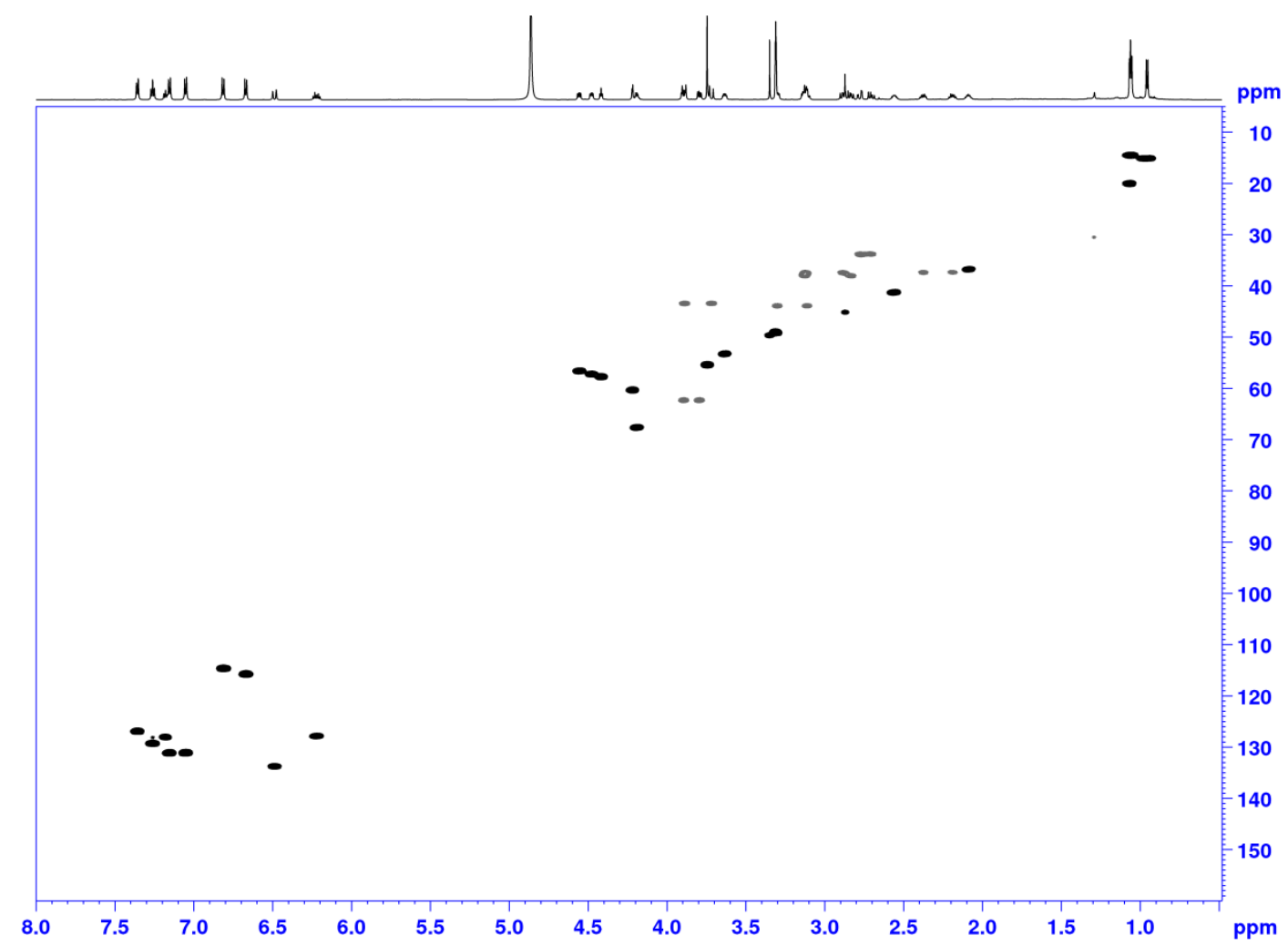

Figure S19: HSQC spectrum of Thaxteramide A1 (CD $\left.{ }_{3} \mathrm{OD}, 700 \mathrm{MHz}\right)$

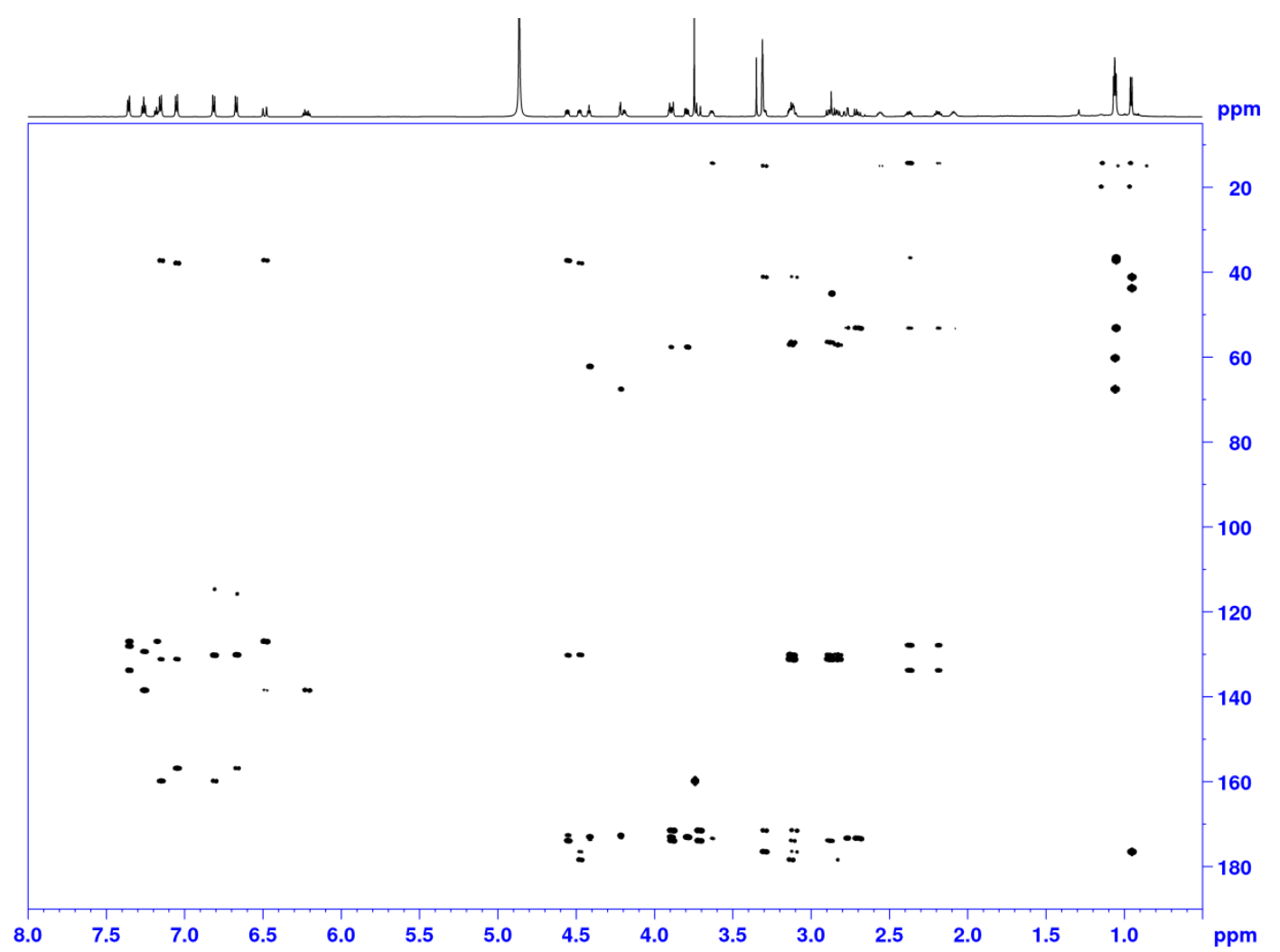

Figure S20: HMBC spectrum of Thaxteramide A1 (CD $\left.{ }_{3} \mathrm{OD}, 700 \mathrm{MHz}\right)$ 


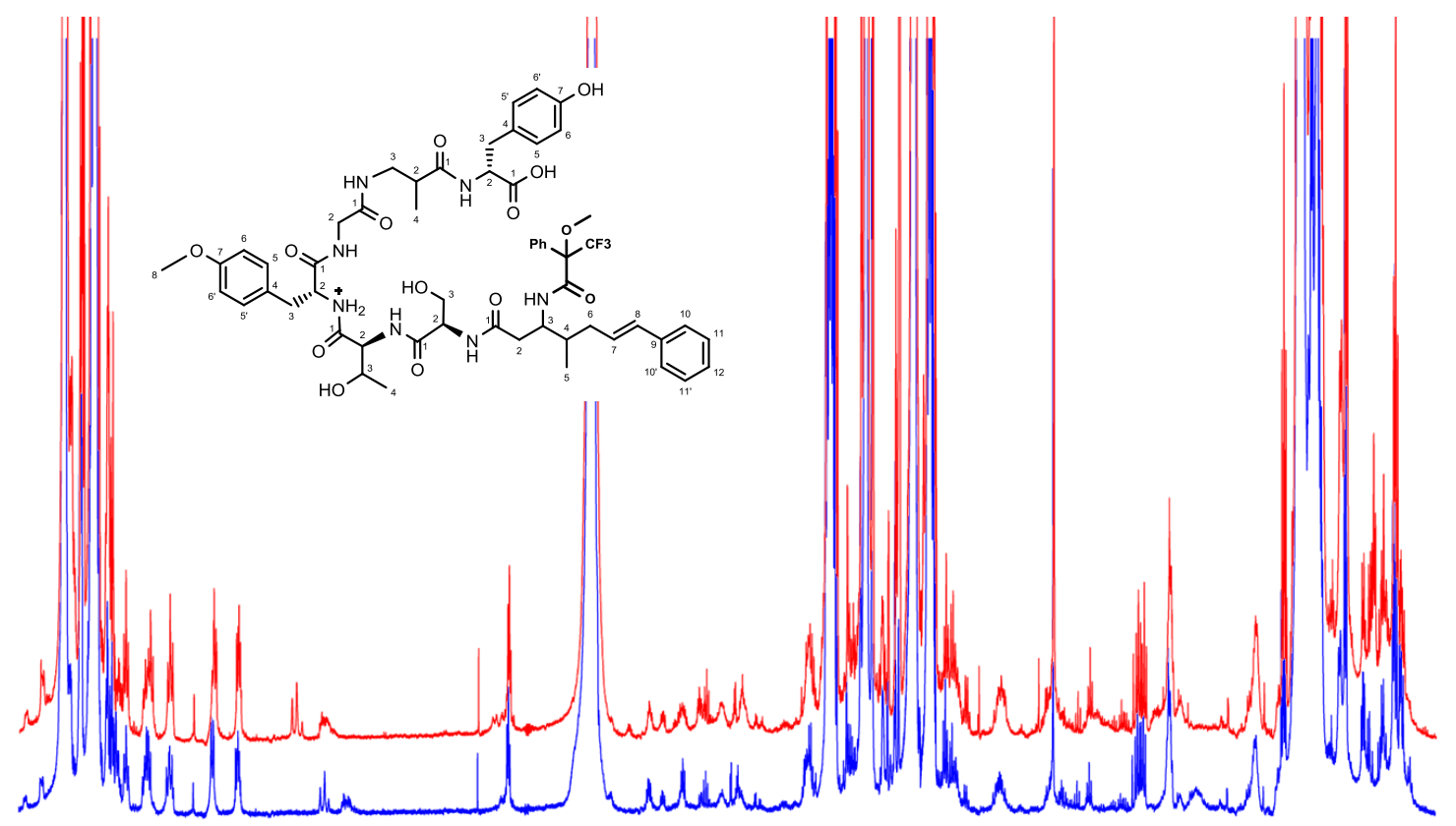

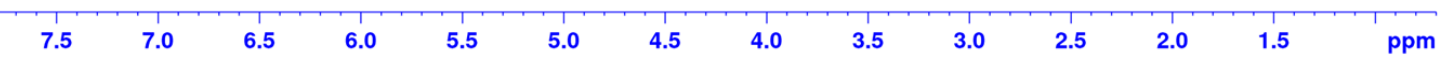

Figure S21: ${ }^{1} \mathrm{H}$ spectra comparison of $(S)$ - (in blue) and $(R)$ - (in red) MTPA amide of ThxA1 $\left(\mathrm{CD}_{3} \mathrm{OD}, 700 \mathrm{MHz}\right)$
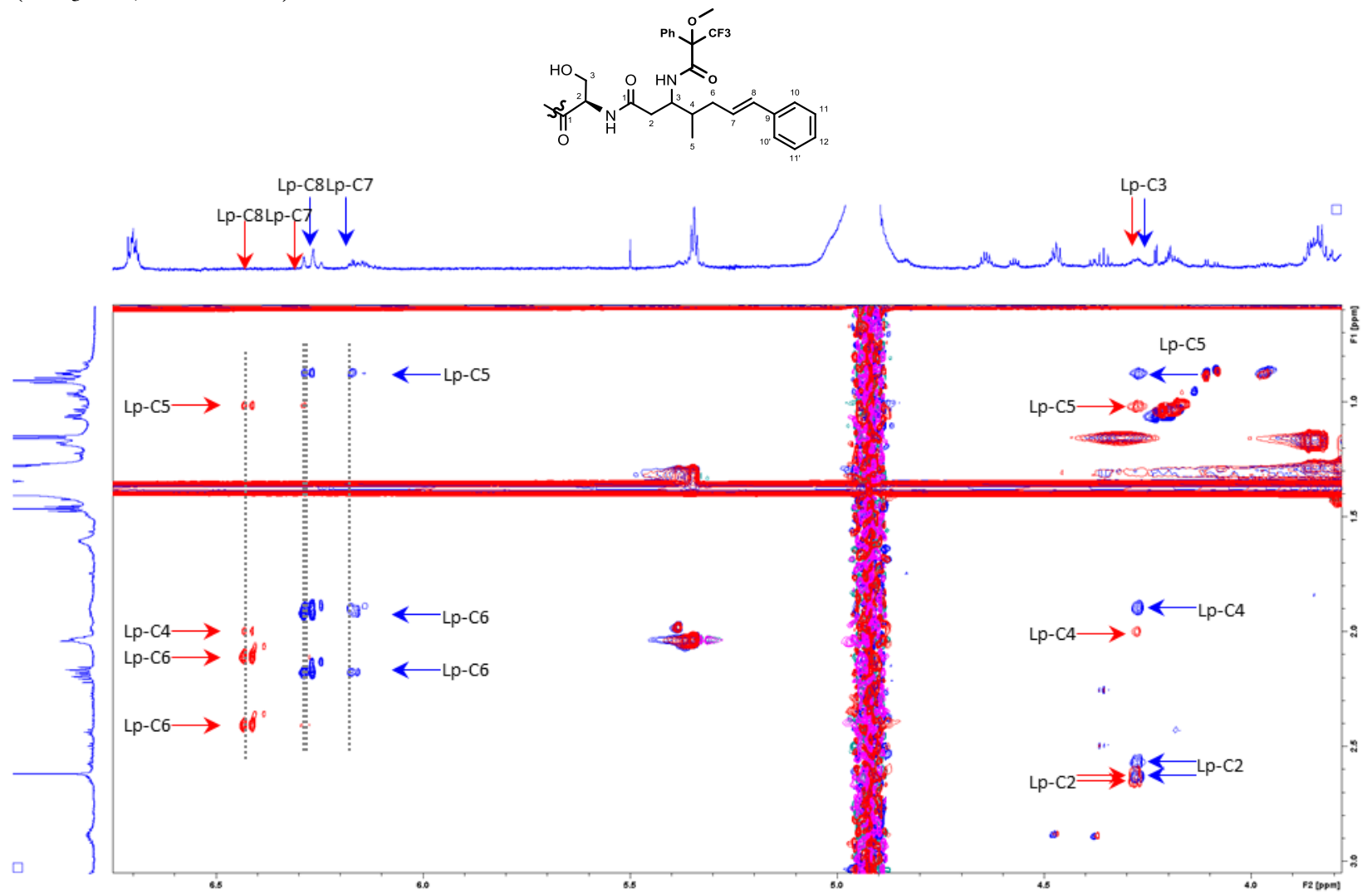

Figure S22: TOCSY spectra comparison of $(S)$ - (in blue) and $(R)$ - (in red) MTPA amide of ThxA1 $\left(\mathrm{CD}_{3} \mathrm{OD}, 700 \mathrm{MHz}\right)$ showing lipid chain 


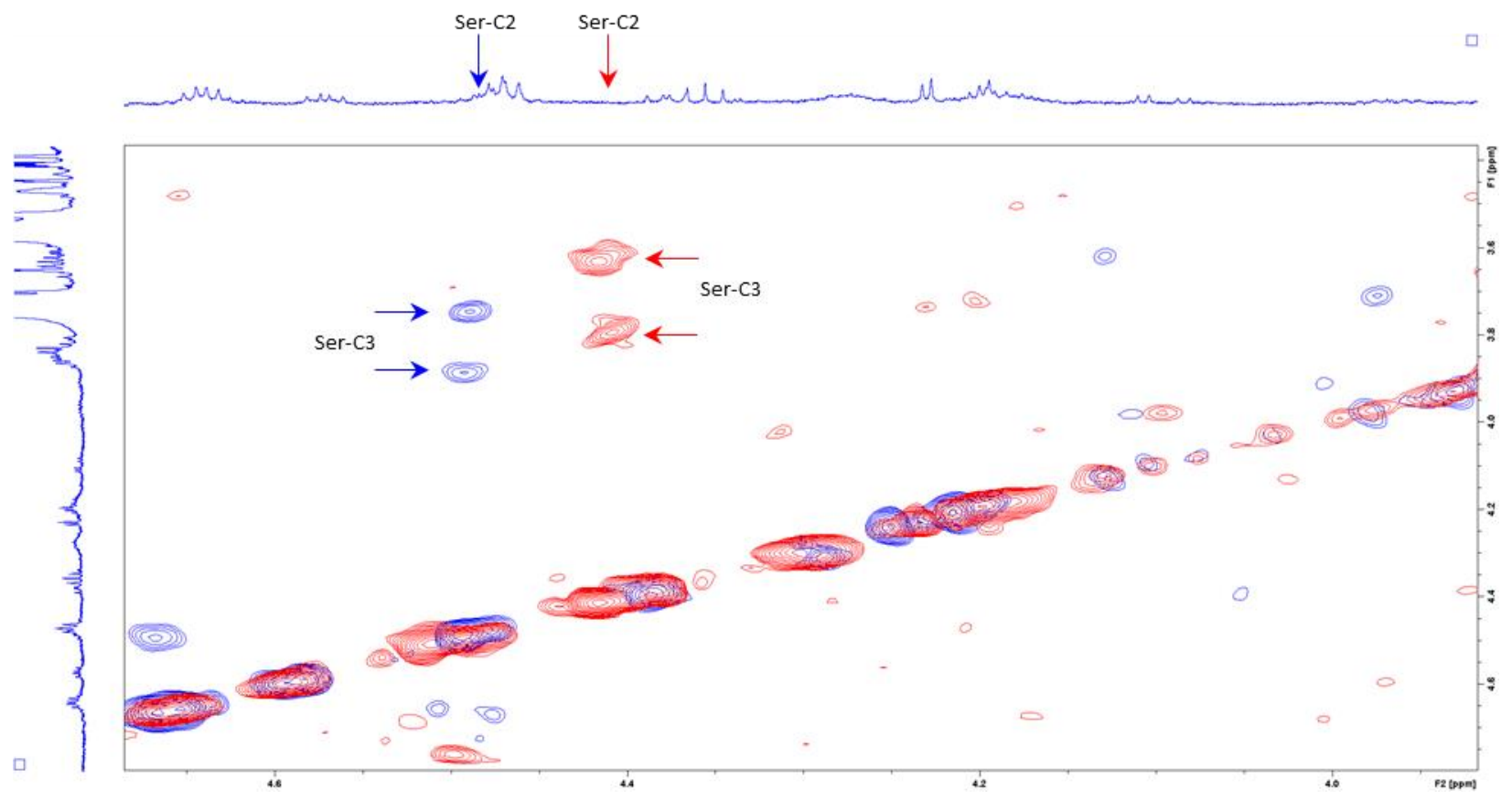

Figure S23: COSY spectra comparison of $(S)$ - (in blue) and $(R)$ - (in red) MTPA amide of ThxA1 $\left(\mathrm{CD}_{3} \mathrm{OD}, 700 \mathrm{MHz}\right)$ showing Serine 


\section{Thaxteramide A2}

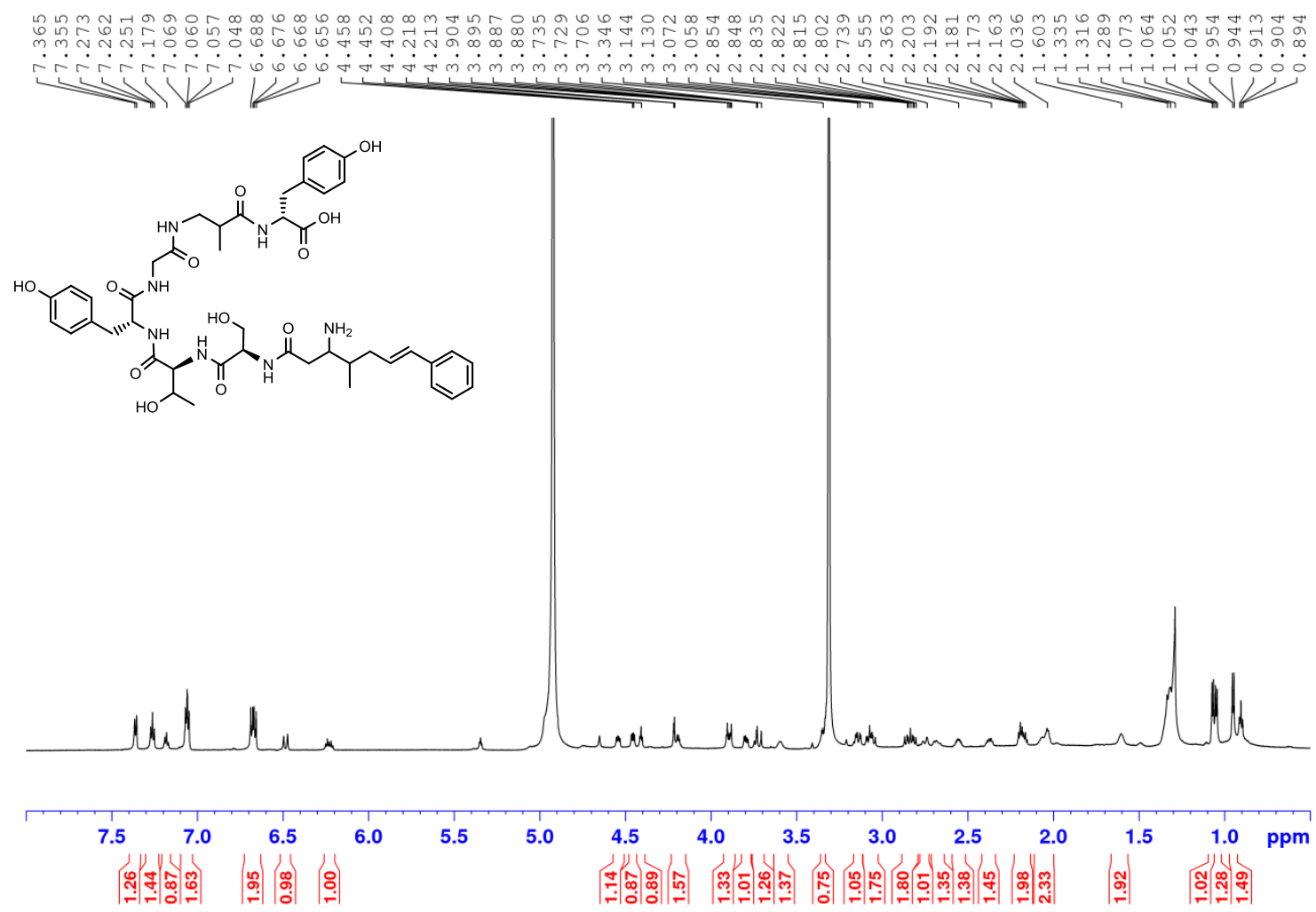

Figure S24: ${ }^{1} \mathrm{H}$ spectrum of Thaxteramide $\mathrm{A} 2\left(\mathrm{CD}_{3} \mathrm{OD}, 700 \mathrm{MHz}\right)$

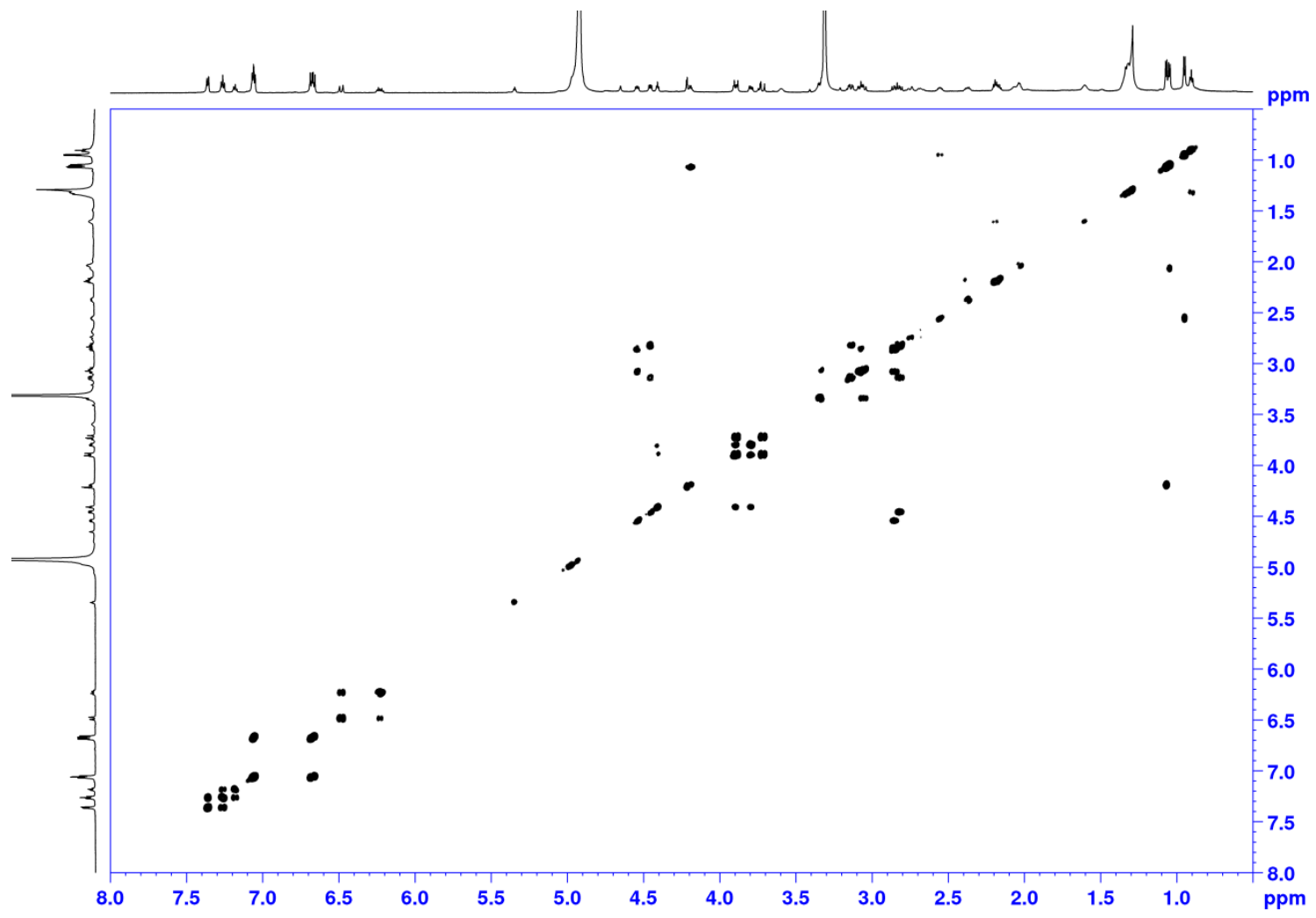

Figure S25: COSY spectrum of Thaxteramide A2 ( $\left.\mathrm{CD}_{3} \mathrm{OD}, 700 \mathrm{MHz}\right)$ 


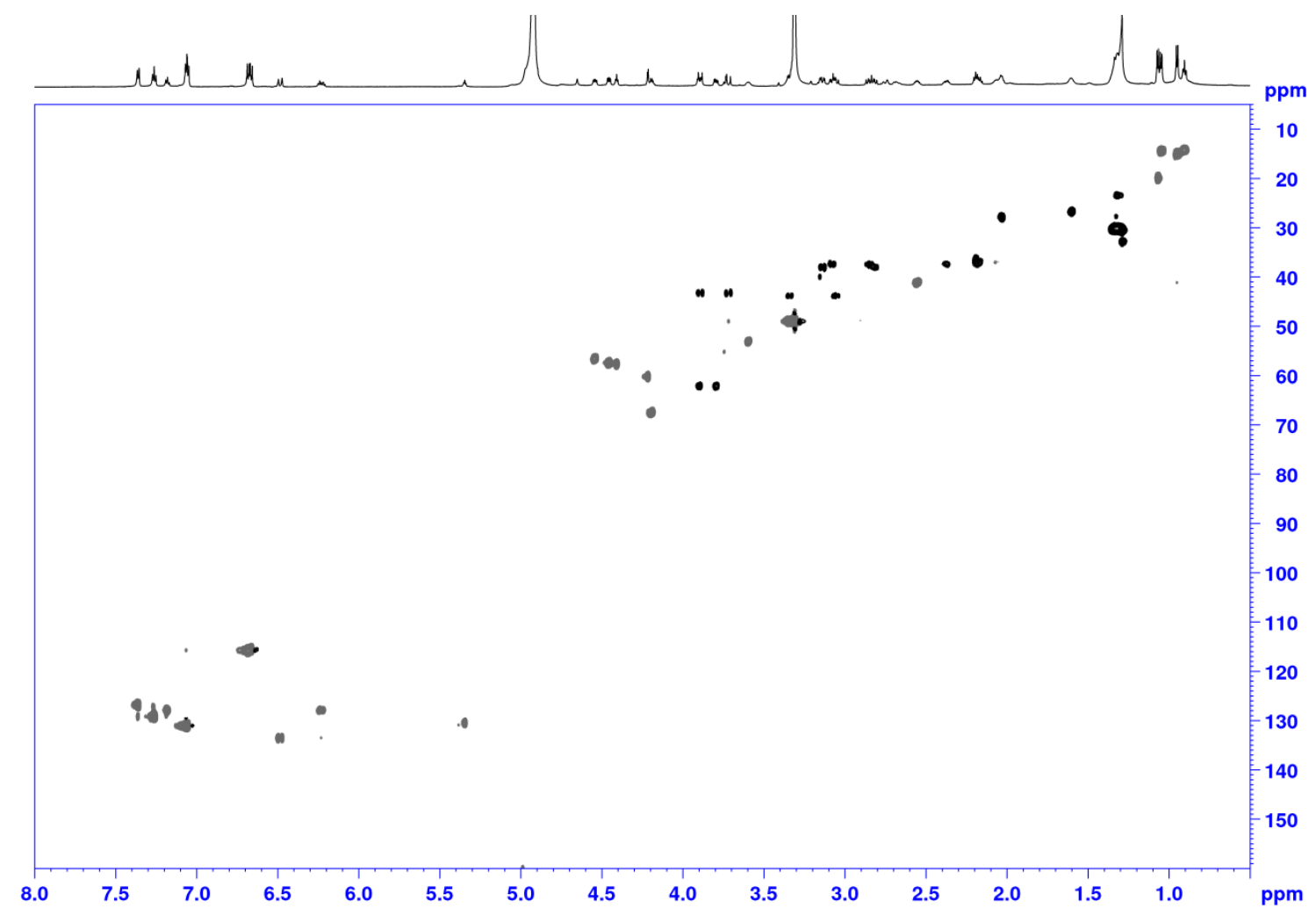

Figure S26: HSQC spectrum of Thaxteramide A2 $\left(\mathrm{CD}_{3} \mathrm{OD}, 700 \mathrm{MHz}\right)$

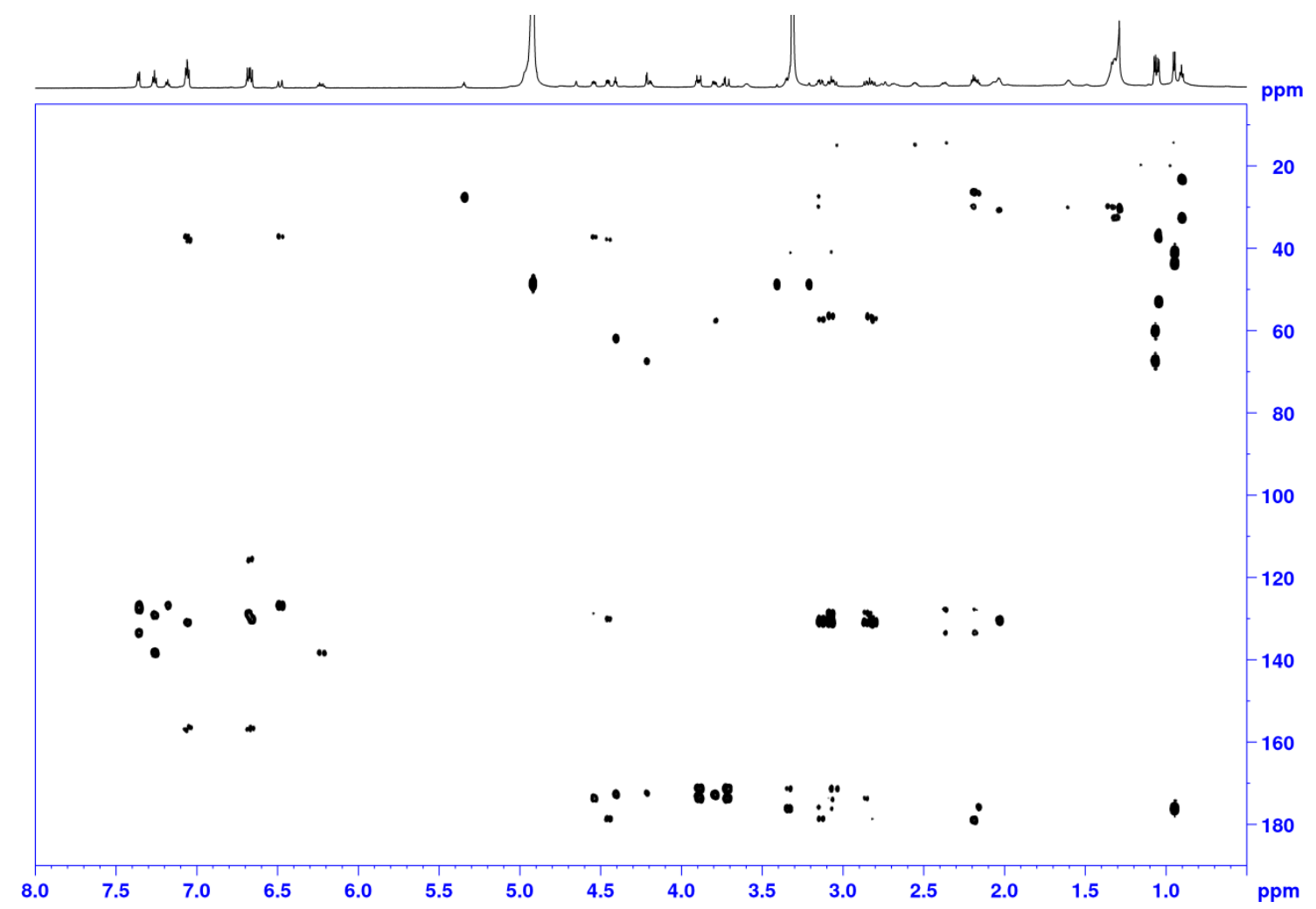

Figure S27: HMBC spectrum of Thaxteramide $\mathrm{A} 2\left(\mathrm{CD}_{3} \mathrm{OD}, 700 \mathrm{MHz}\right)$ 


\section{Thaxteramide B1}

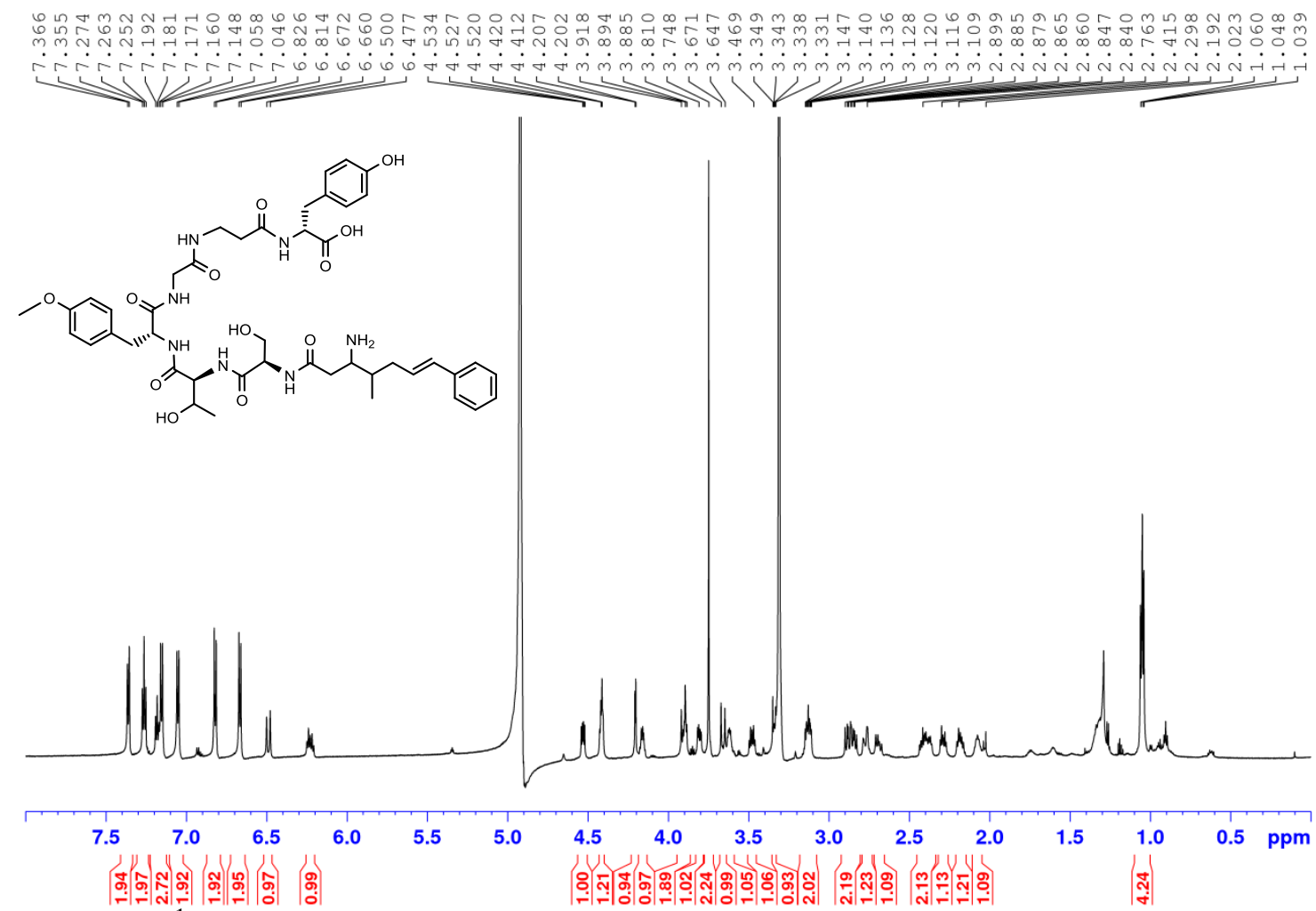

Figure S28: ${ }^{1} \mathrm{H}$ spectrum of Thaxteramide $\mathrm{B} 1\left(\mathrm{CD}_{3} \mathrm{OD}, 700 \mathrm{MHz}\right)$

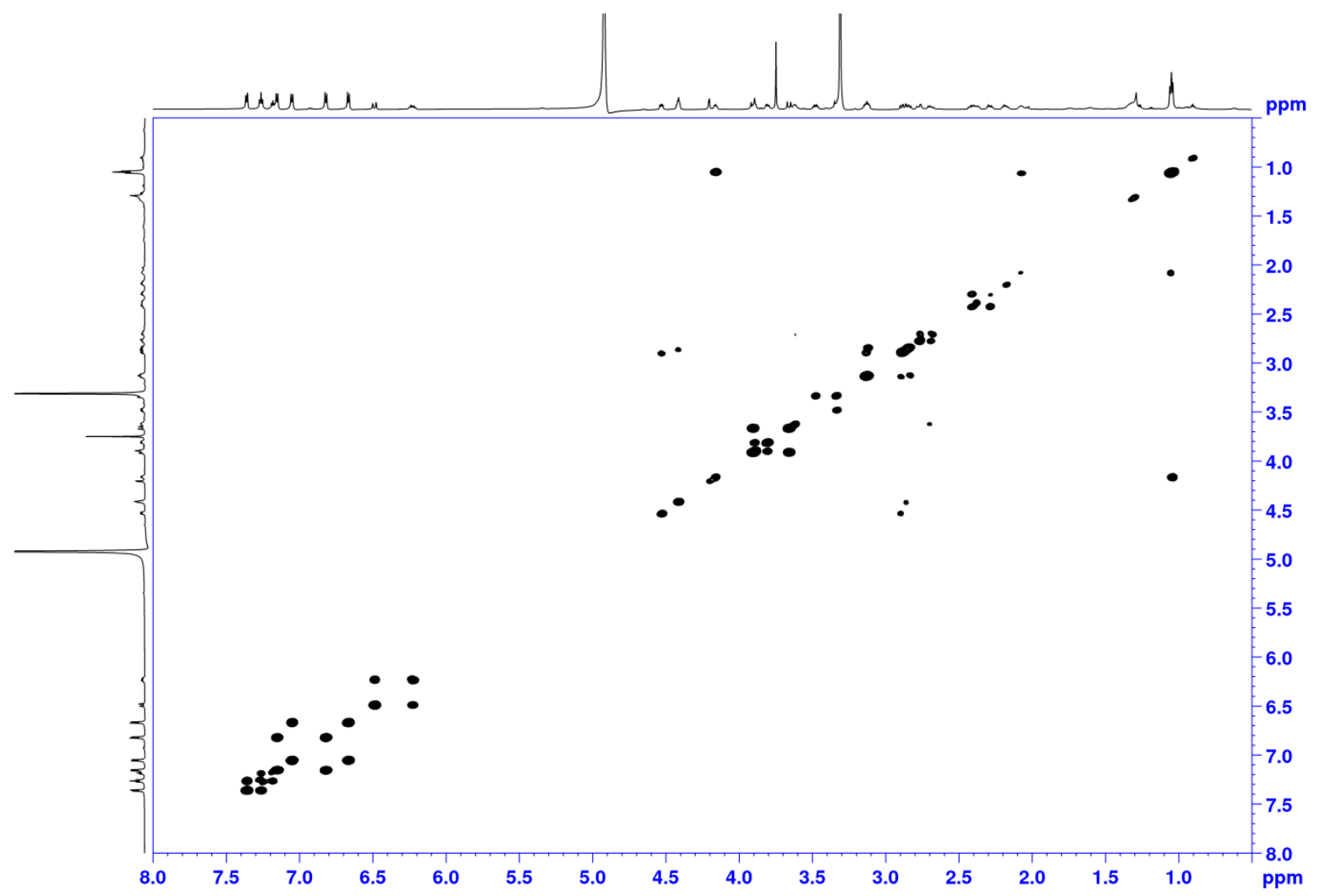

Figure S29: COSY spectrum of Thaxteramide $\mathrm{B} 1\left(\mathrm{CD}_{3} \mathrm{OD}, 700 \mathrm{MHz}\right)$ 


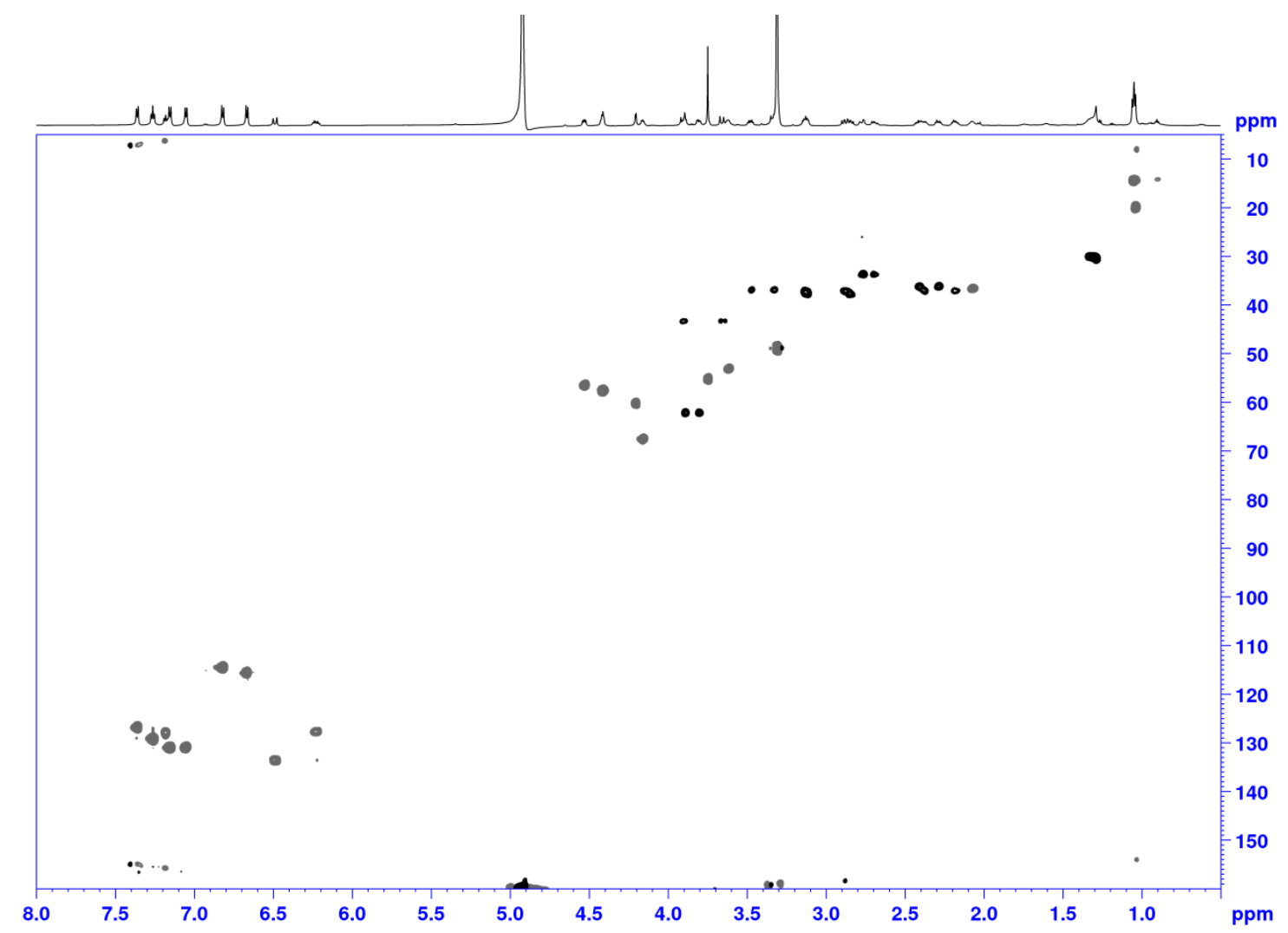

Figure S30: HSQC spectrum of Thaxteramide B1 $\left(\mathrm{CD}_{3} \mathrm{OD}, 700 \mathrm{MHz}\right)$

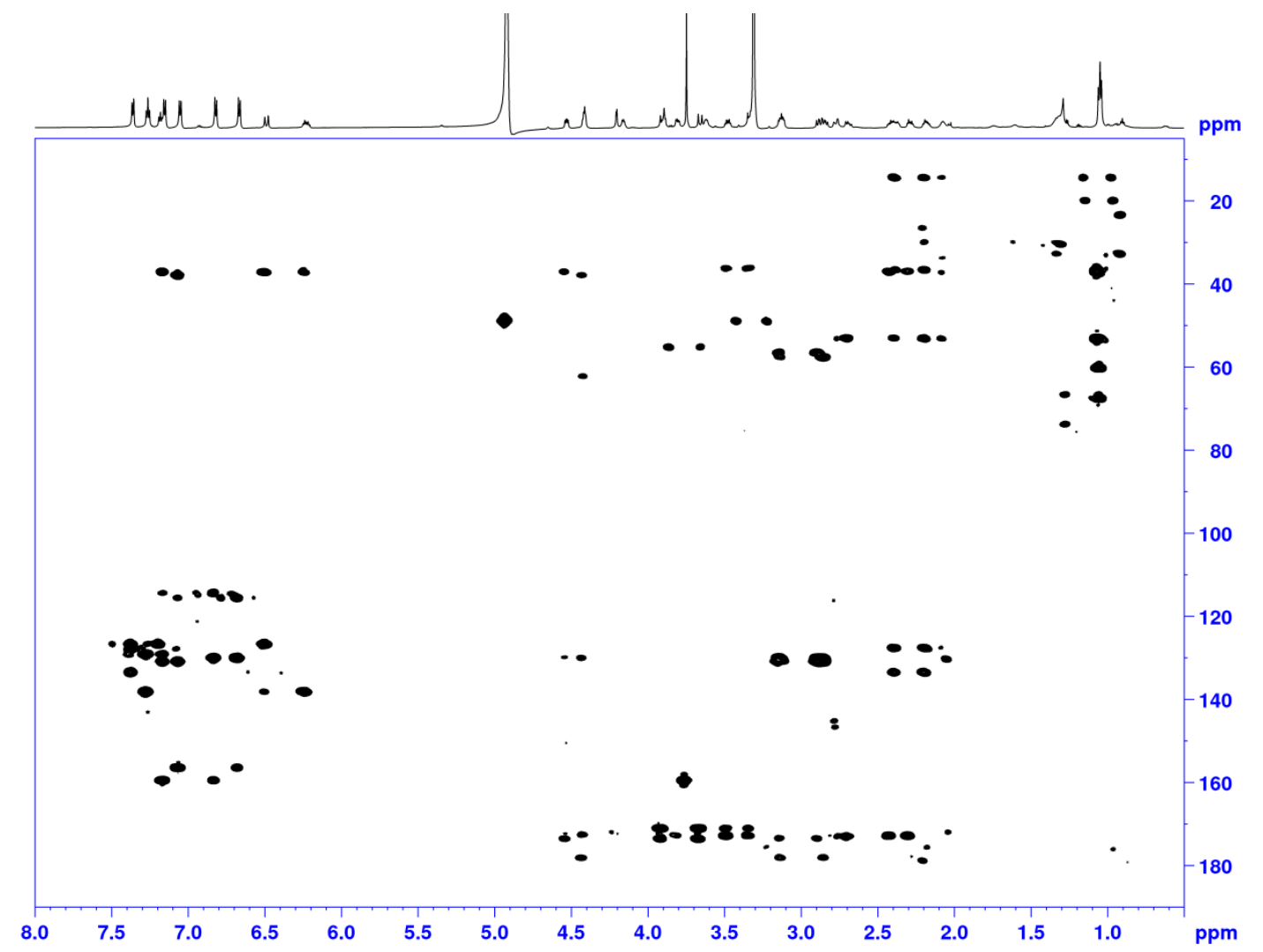

Figure S31: HMBC spectrum of Thaxteramide B1 $\left(\mathrm{CD}_{3} \mathrm{OD}, 700 \mathrm{MHz}\right)$ 


\section{Thaxteramide B2}

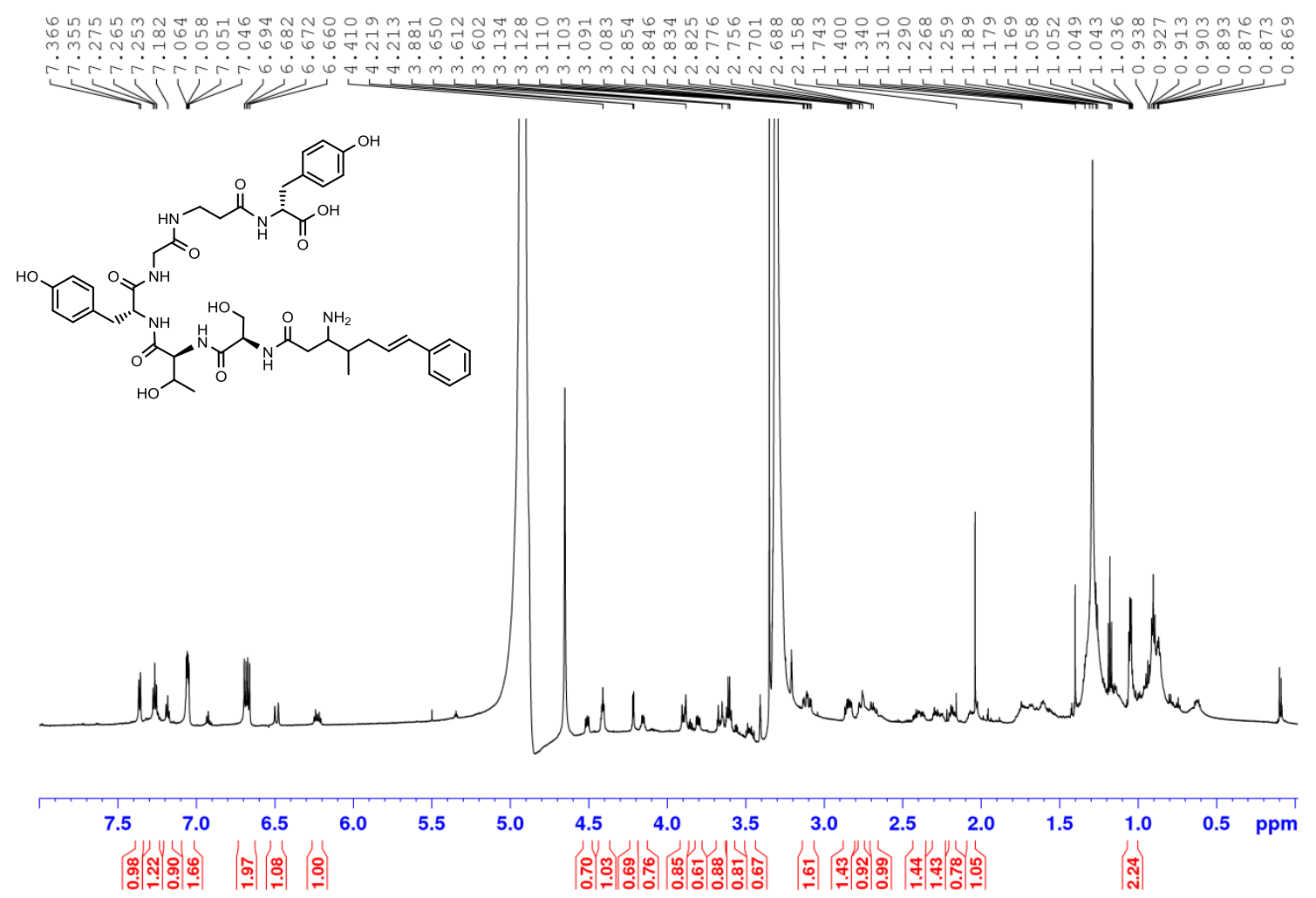

Figure S32: ${ }^{1} \mathrm{H}$ spectrum of Thaxteramide $\mathrm{B} 2\left(\mathrm{CD}_{3} \mathrm{OD}, 700 \mathrm{MHz}\right)$

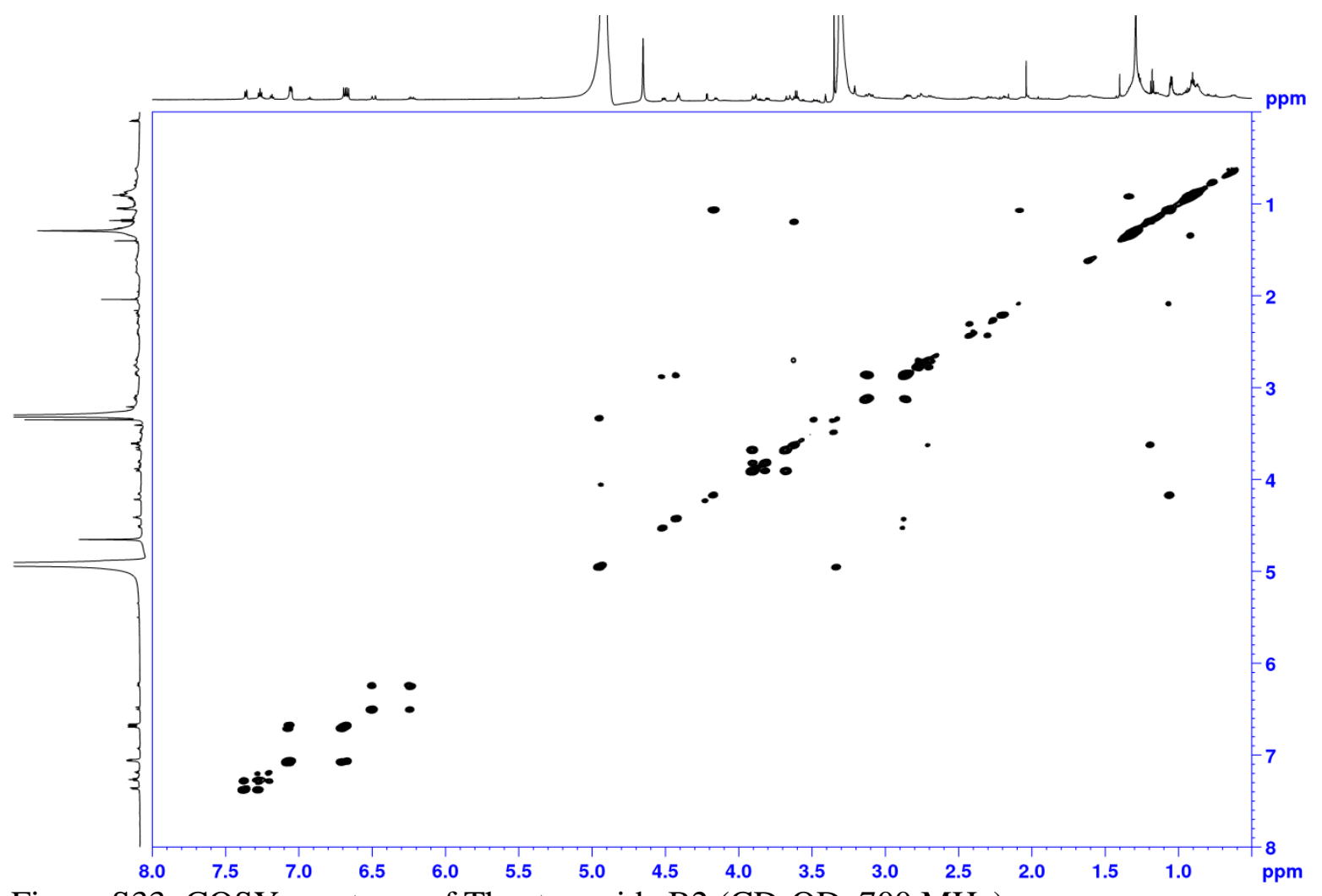

Figure S33: COSY spectrum of Thaxteramide $\mathrm{B} 2\left(\mathrm{CD}_{3} \mathrm{OD}, 700 \mathrm{MHz}\right)$ 

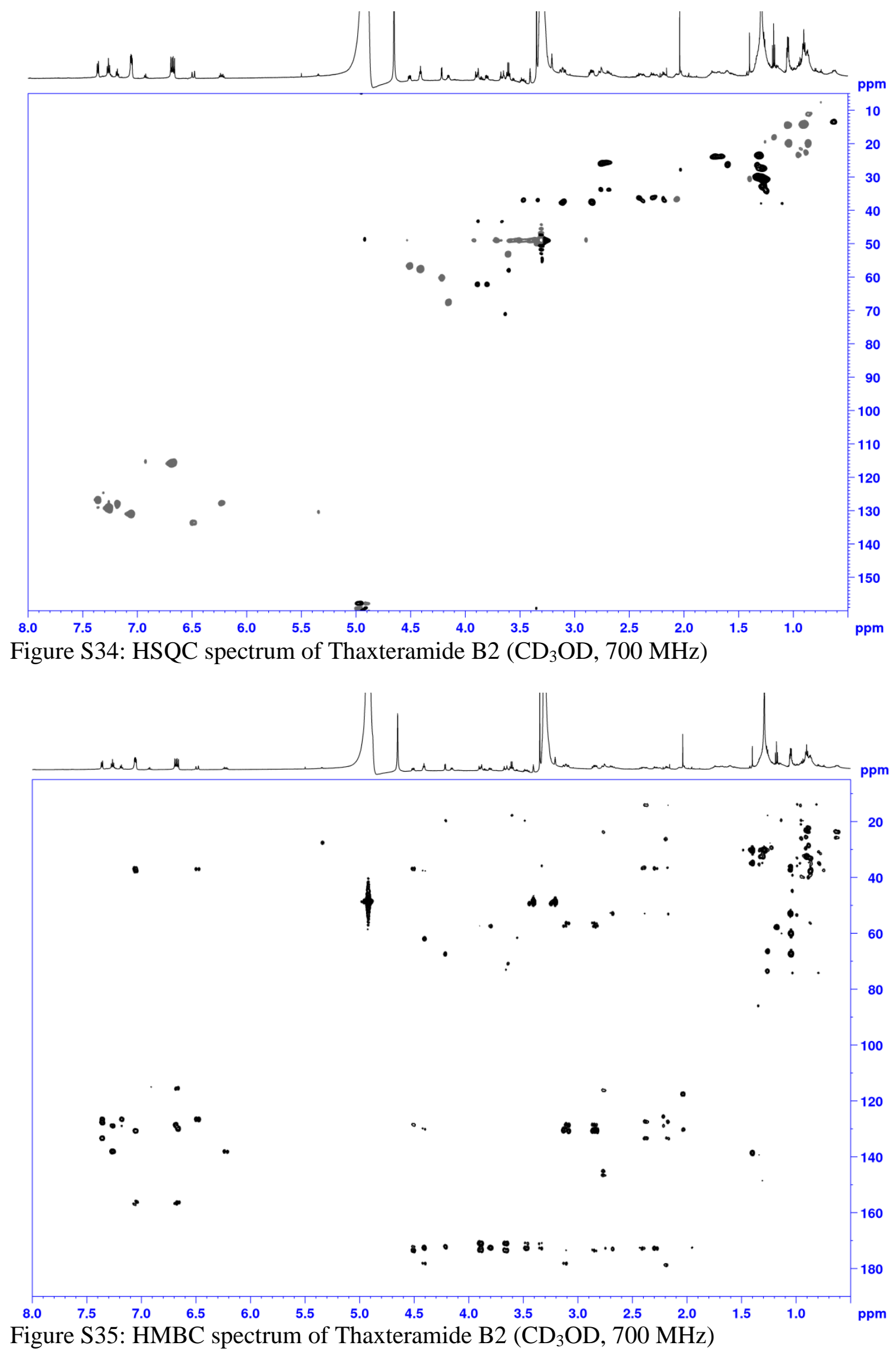


\section{Thaxteramide C}

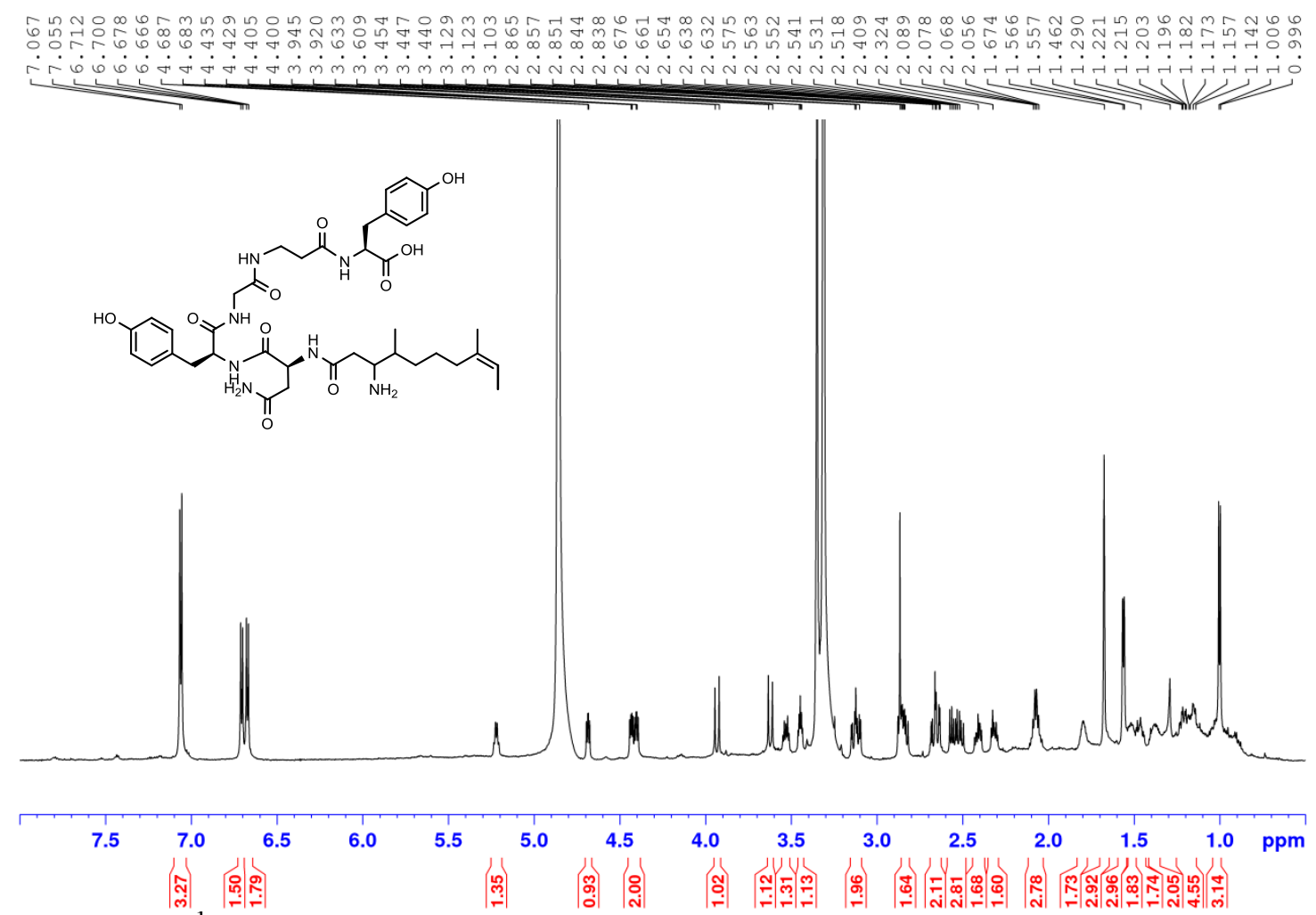

Figure S36: ${ }^{1} \mathrm{H}$ spectrum of Thaxteramide $\mathrm{C}\left(\mathrm{CD}_{3} \mathrm{OD}, 700 \mathrm{MHz}\right)$

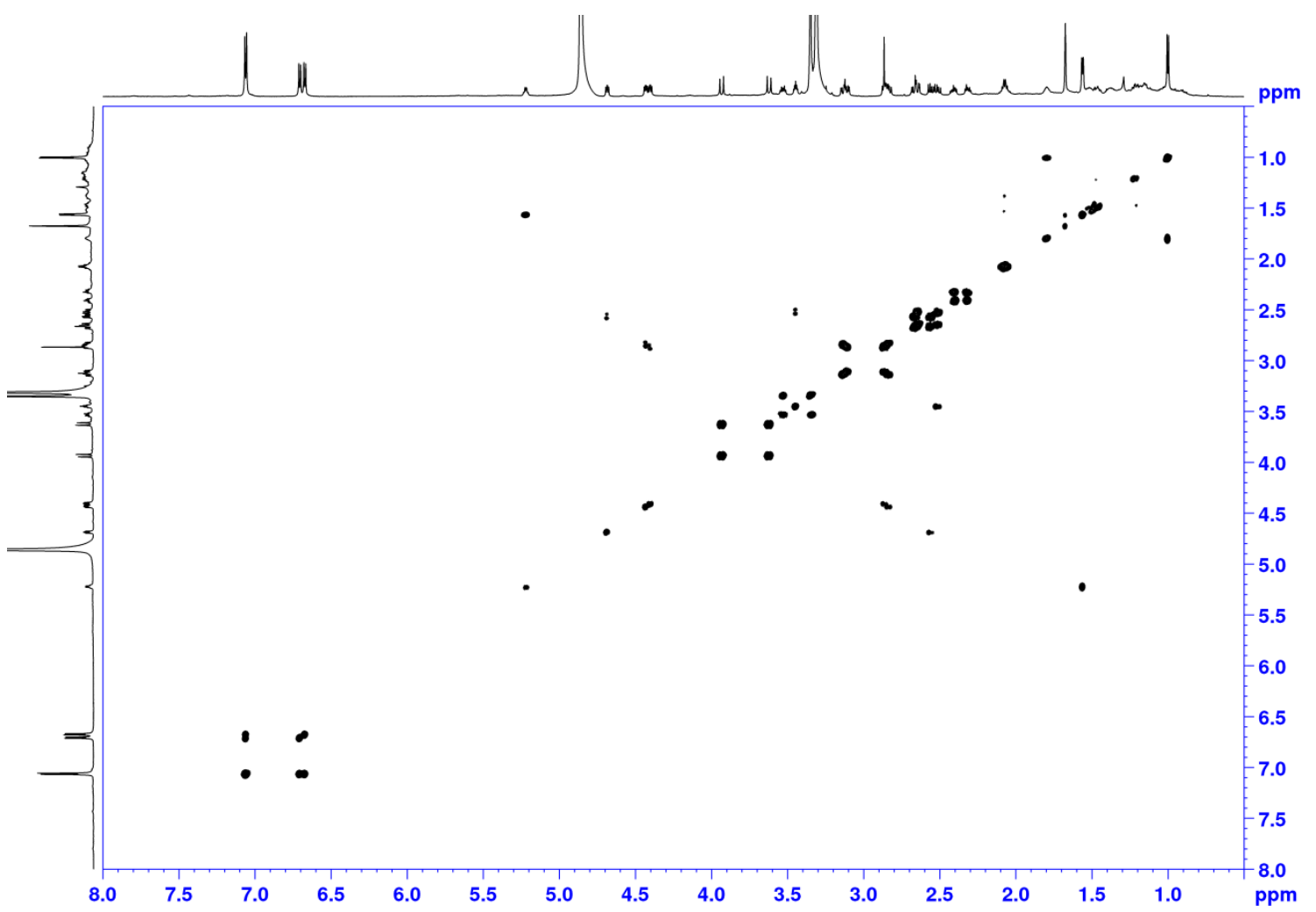

Figure S37: COSY spectrum of Thaxteramide $\mathrm{C}\left(\mathrm{CD}_{3} \mathrm{OD}, 700 \mathrm{MHz}\right)$ 


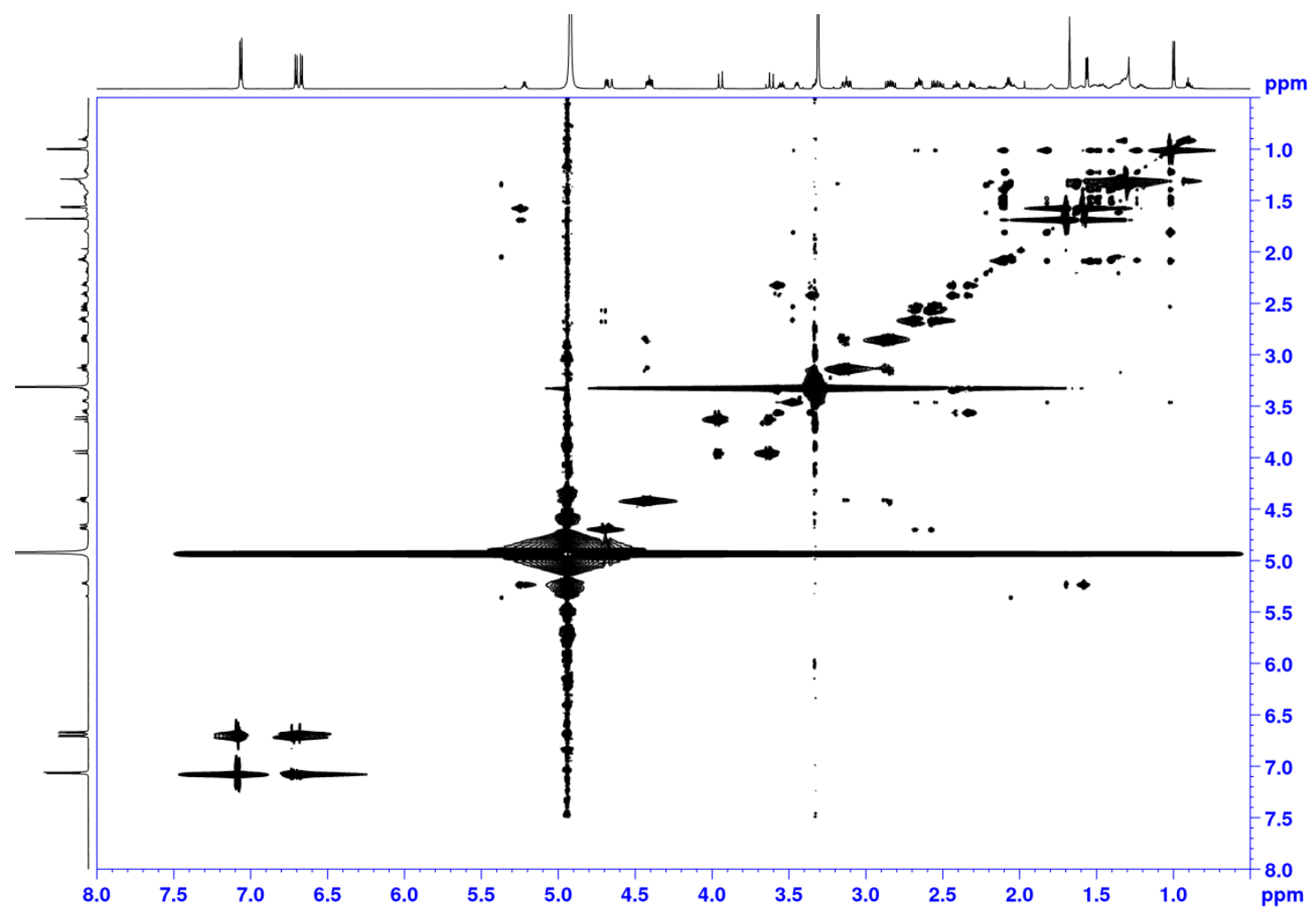

Figure S38: TOCSY spectrum of Thaxteramide $\mathrm{C}\left(\mathrm{CD}_{3} \mathrm{OD}, 700 \mathrm{MHz}\right)$

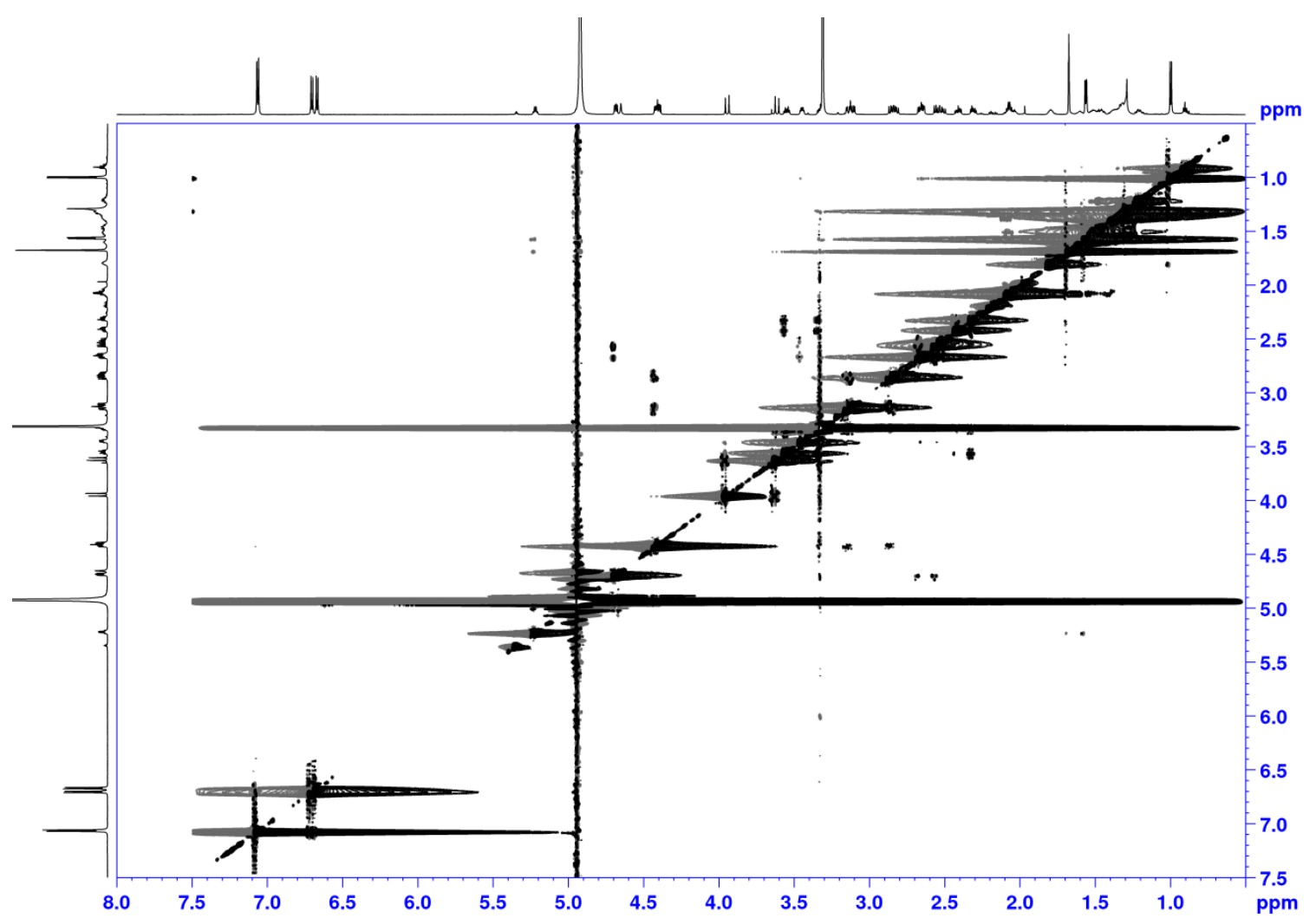

Figure S39: ROESY spectrum of Thaxteramide $\mathrm{C}\left(\mathrm{CD}_{3} \mathrm{OD}, 700 \mathrm{MHz}\right)$ 


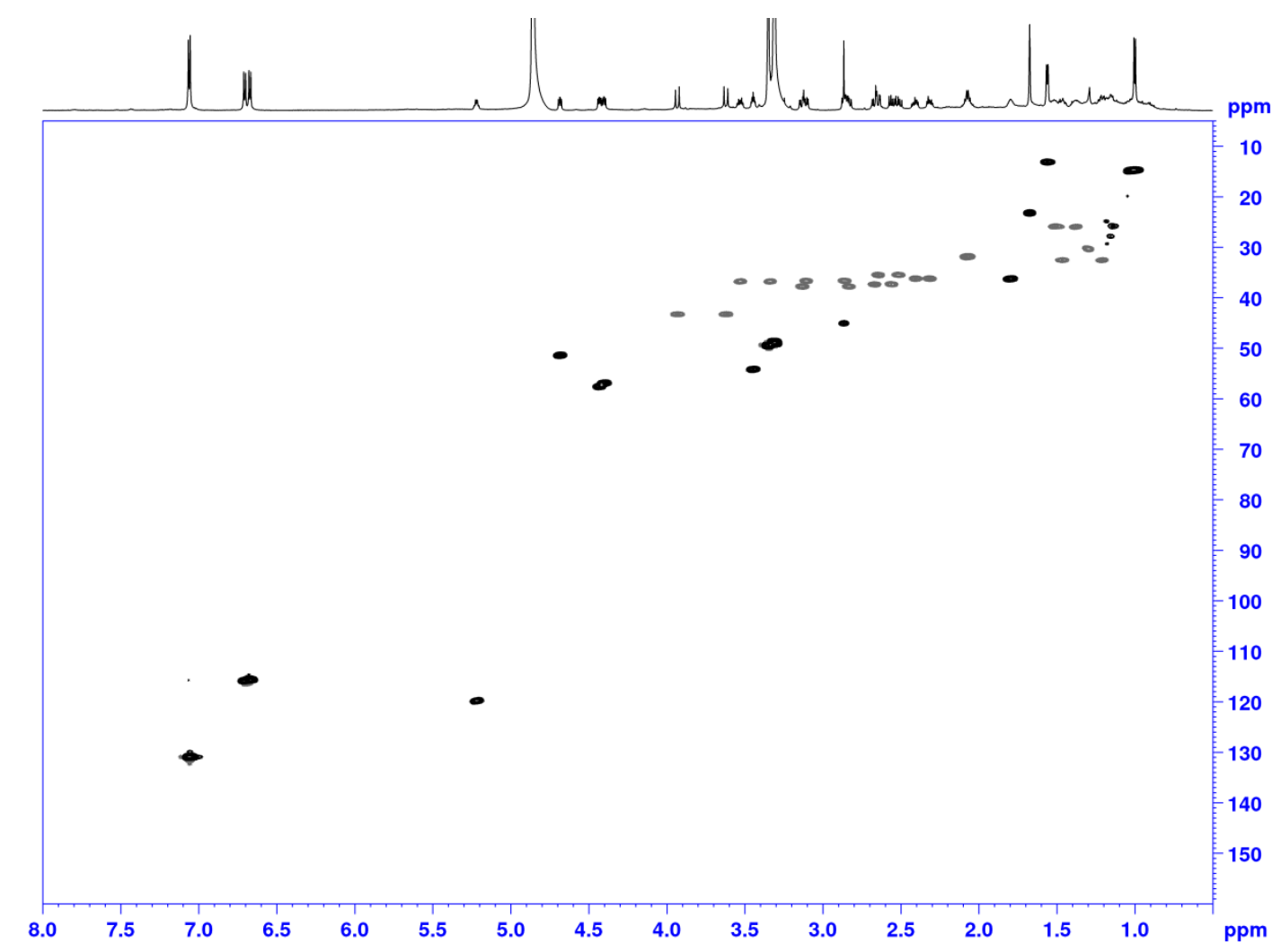

Figure S40: HSQC spectrum of Thaxteramide C $\left(\mathrm{CD}_{3} \mathrm{OD}, 700 \mathrm{MHz}\right)$

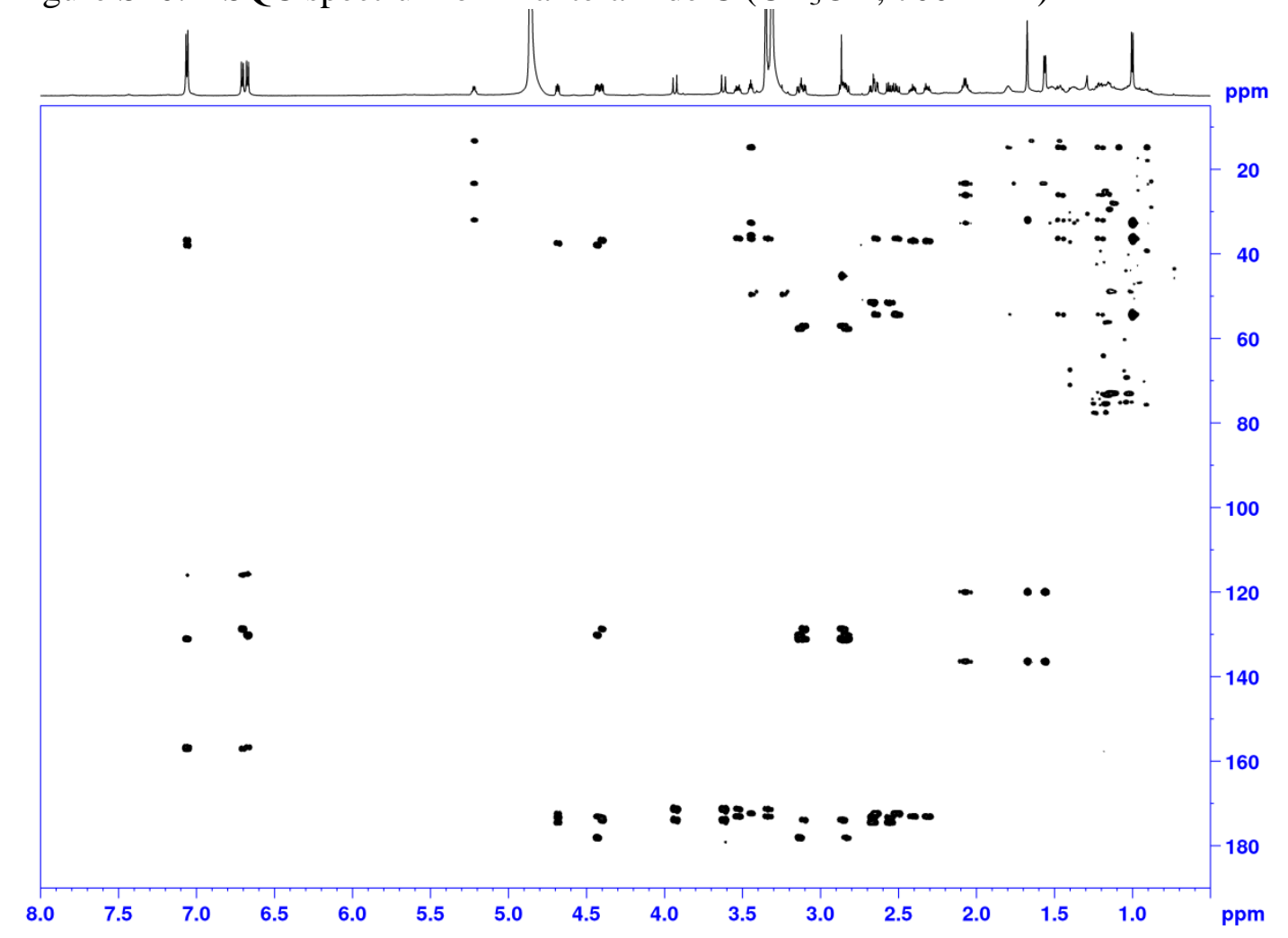

Figure S41: HMBC spectrum of Thaxteramide C $\left(\mathrm{CD}_{3} \mathrm{OD}, 700 \mathrm{MHz}\right)$ 


\section{Thaxteramide D}

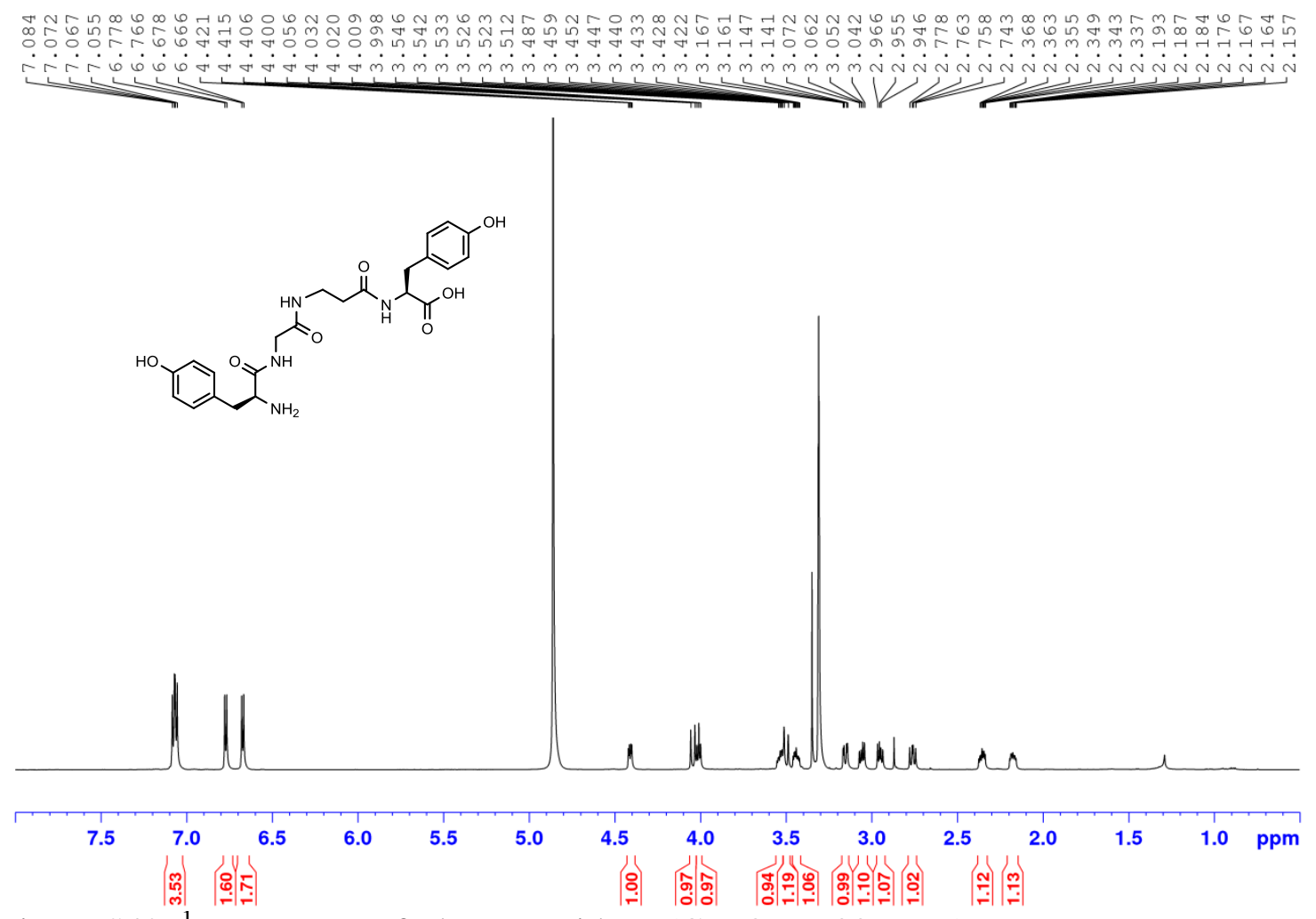

Figure S42: ${ }^{1} \mathrm{H}$ spectrum of Thaxteramide $\mathrm{D}\left(\mathrm{CD}_{3} \mathrm{OD}, 700 \mathrm{MHz}\right)$

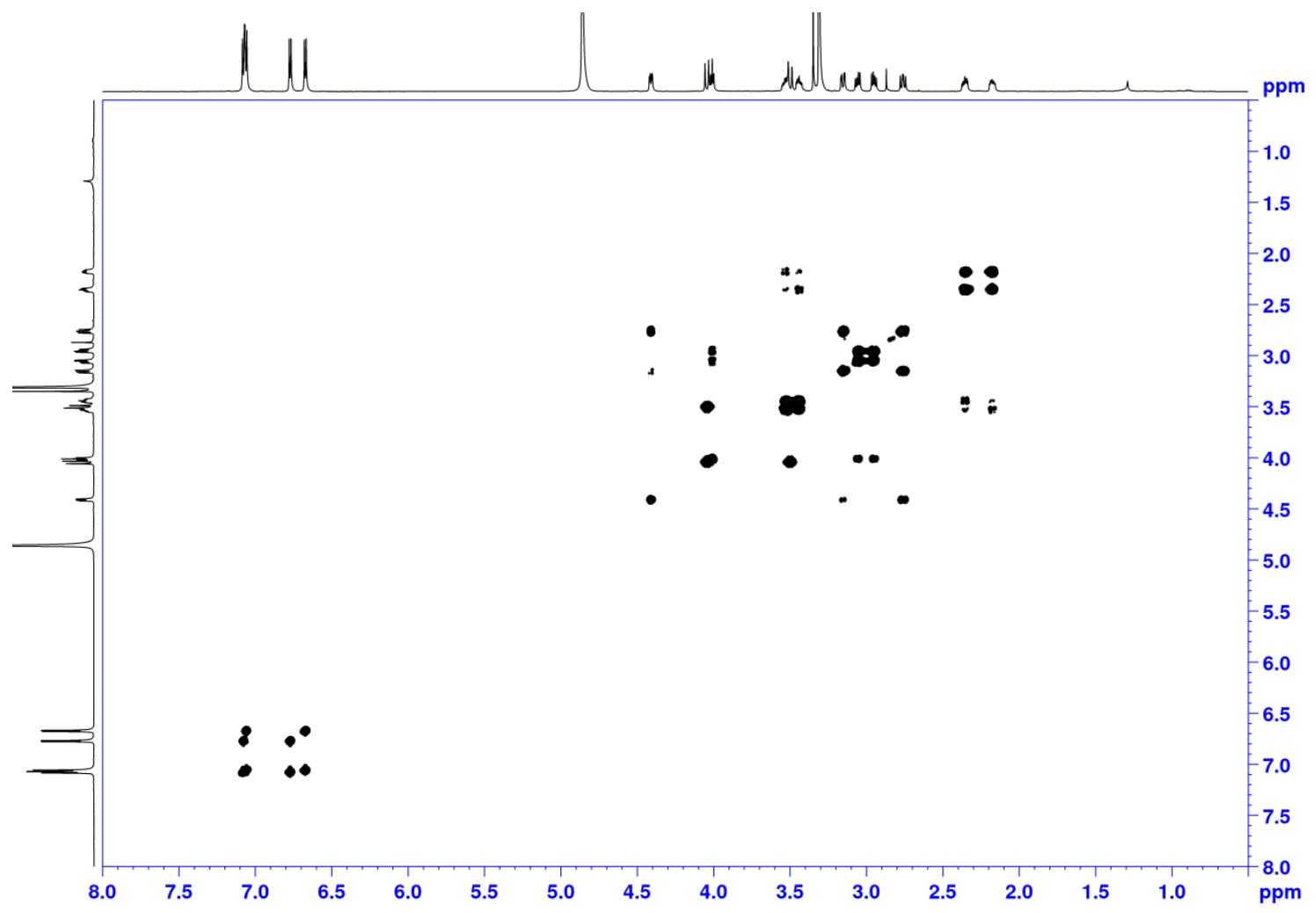

Figure S43: COSY spectrum of Thaxteramide $\mathrm{D}\left(\mathrm{CD}_{3} \mathrm{OD}, 700 \mathrm{MHz}\right)$ 


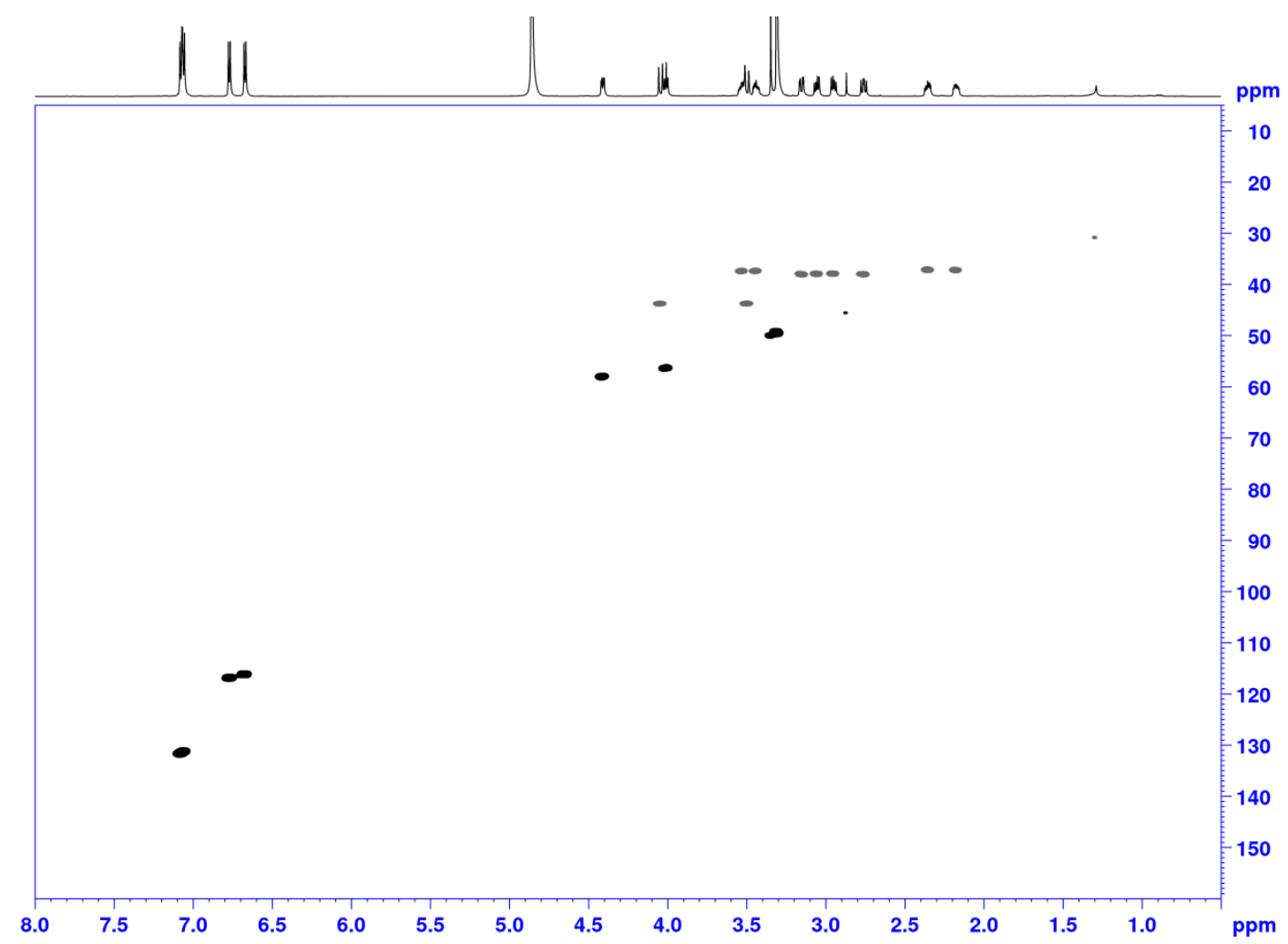

Figure S44: HSQC spectrum of Thaxteramide D $\left(\mathrm{CD}_{3} \mathrm{OD}, 700 \mathrm{MHz}\right)$

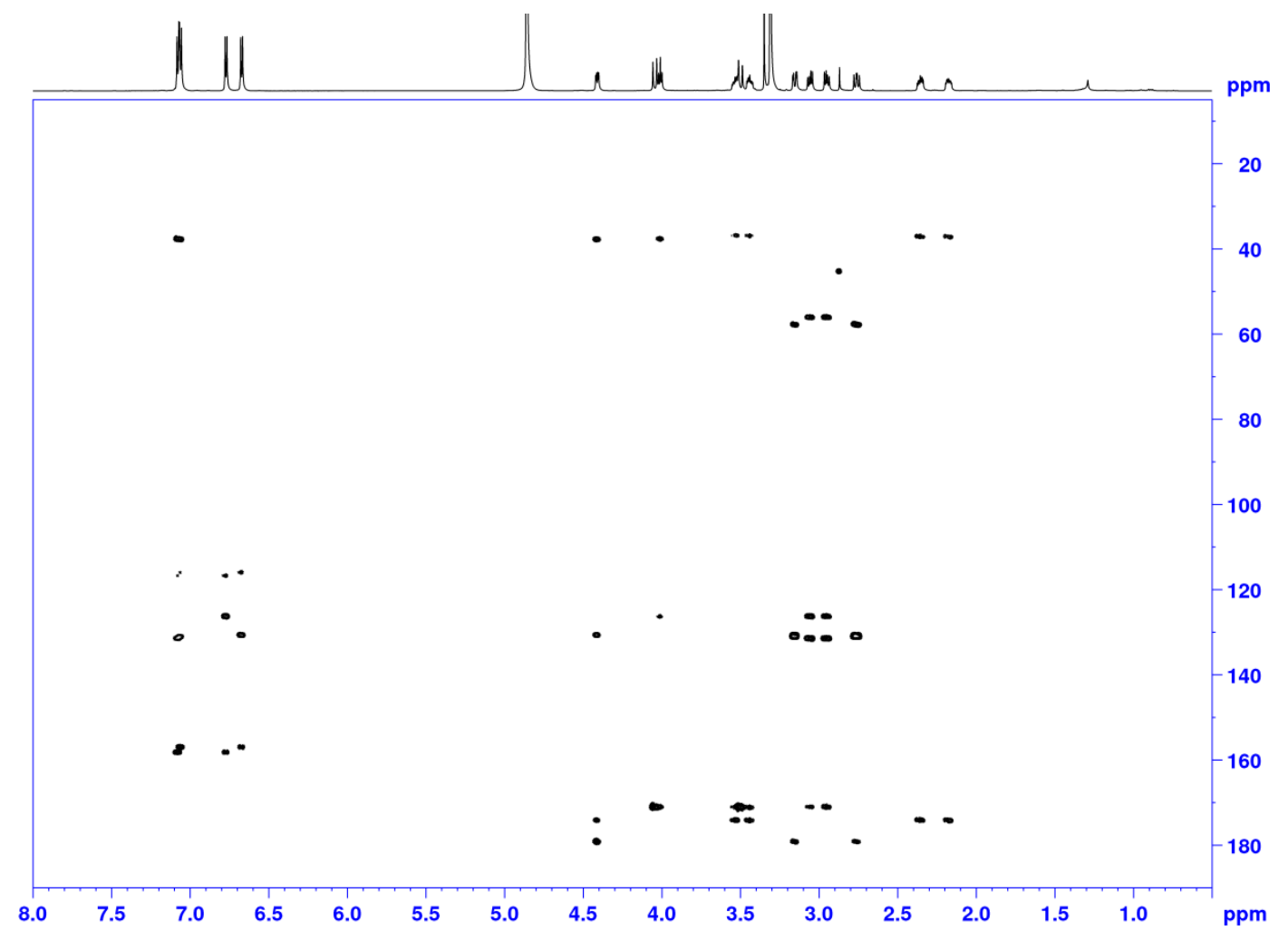

Figure S45: HMBC spectrum of Thaxteramide D $\left(\mathrm{CD}_{3} \mathrm{OD}, 700 \mathrm{MHz}\right)$ 


\section{Cyc-Thaxteramide A}

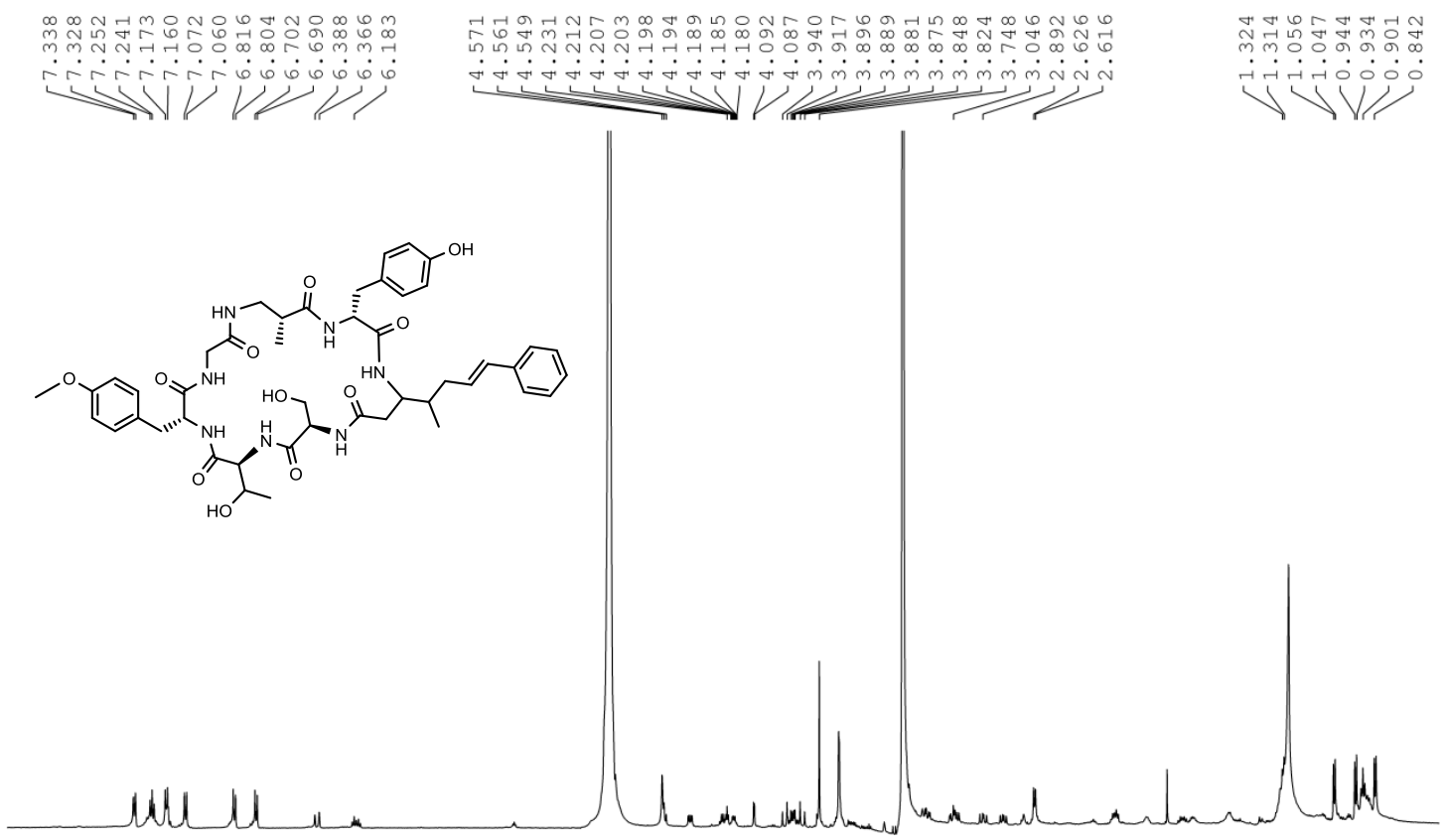

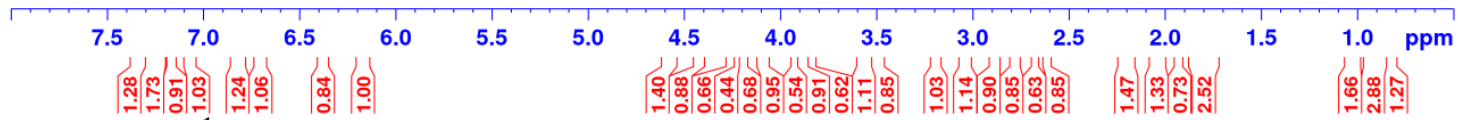

Figure S46: ${ }^{1} \mathrm{H}$ spectrum of Cyc-Thaxteramide $\mathrm{A}\left(\mathrm{CD}_{3} \mathrm{OD}, 700 \mathrm{MHz}\right)$

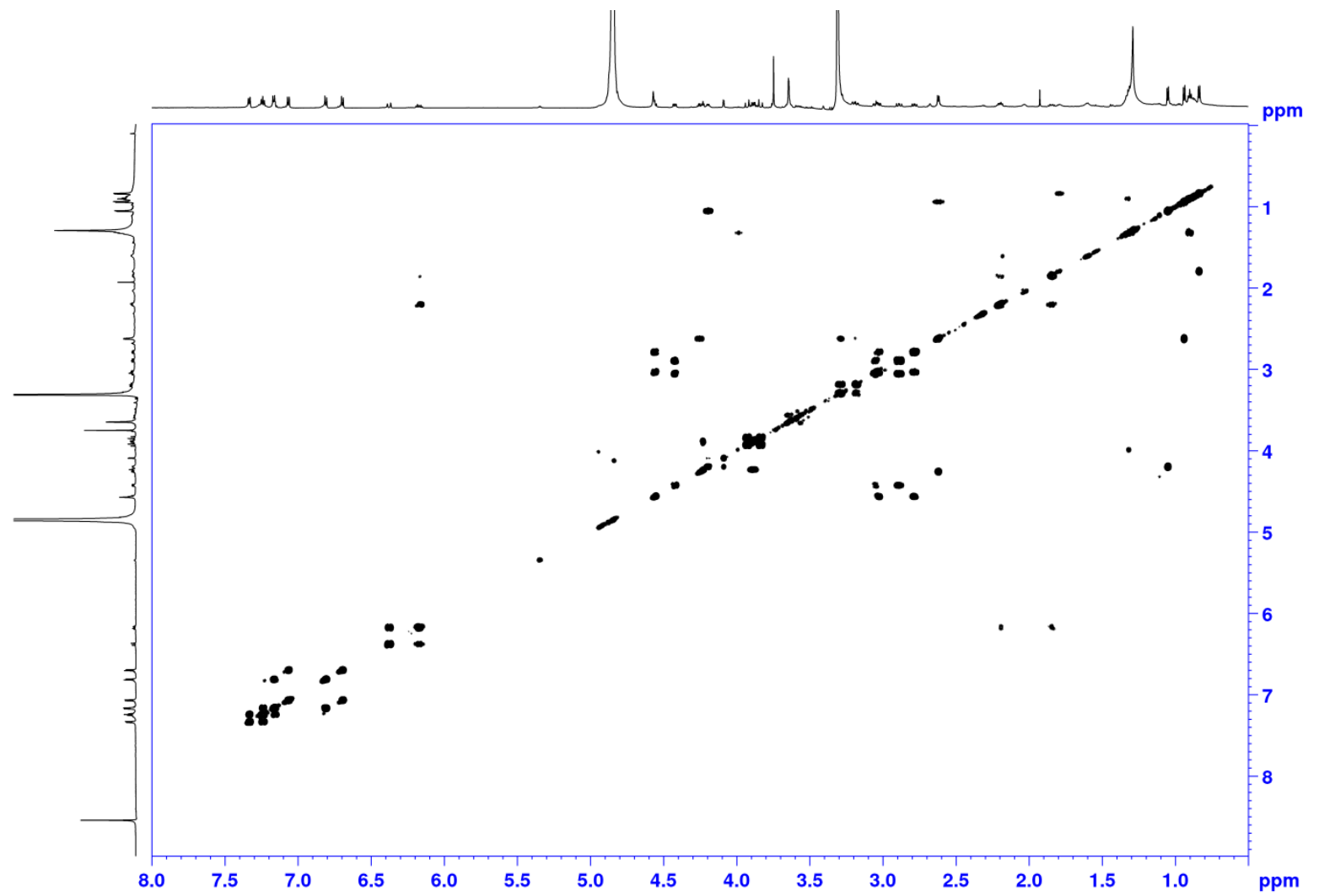

Figure S47: COSY spectrum of Cyc-Thaxteramide $\mathrm{A}\left(\mathrm{CD}_{3} \mathrm{OD}, 700 \mathrm{MHz}\right)$ 


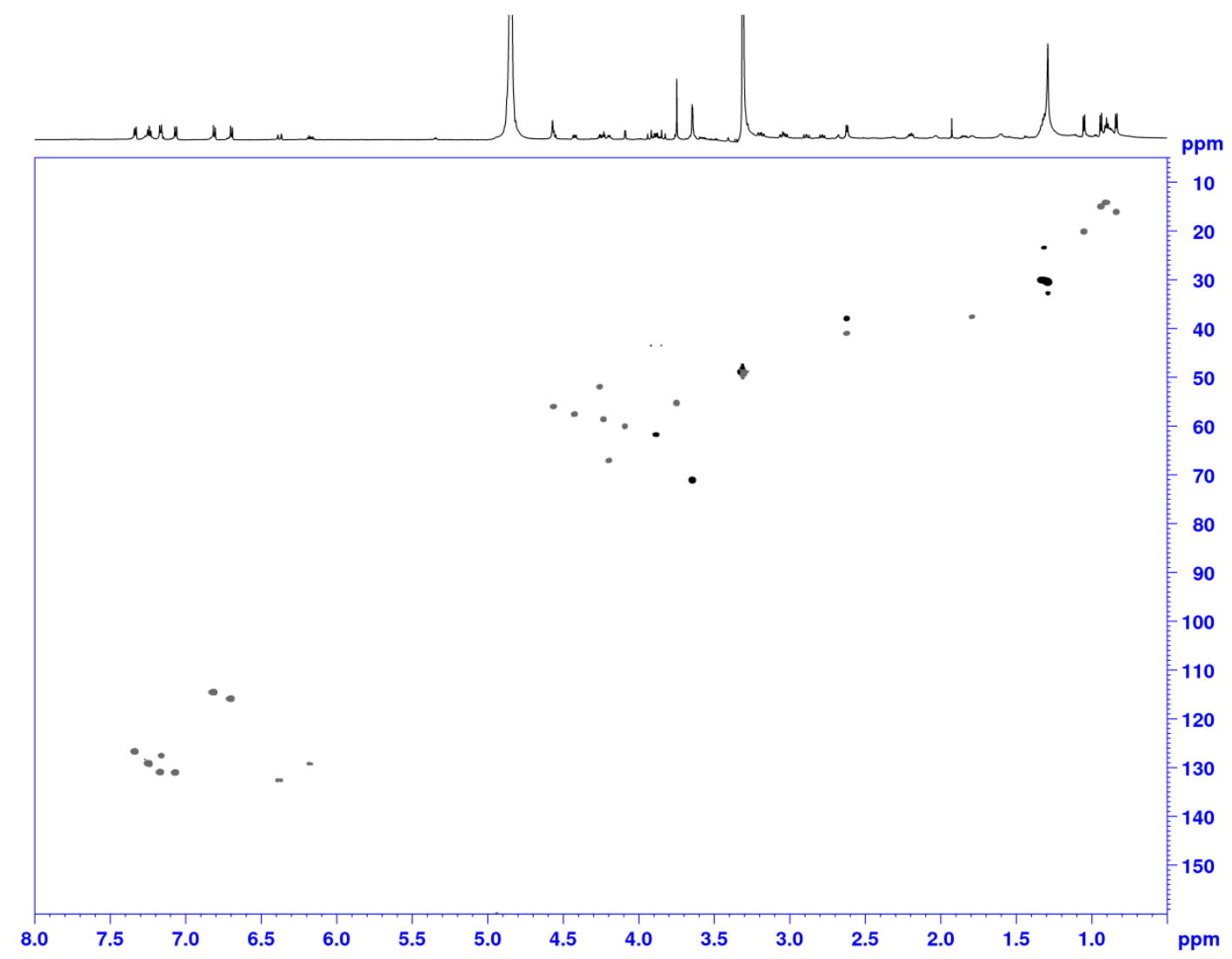

Figure S48: HSQC spectrum of Cyc-Thaxteramide $\mathrm{A}\left(\mathrm{CD}_{3} \mathrm{OD}, 700 \mathrm{MHz}\right)$

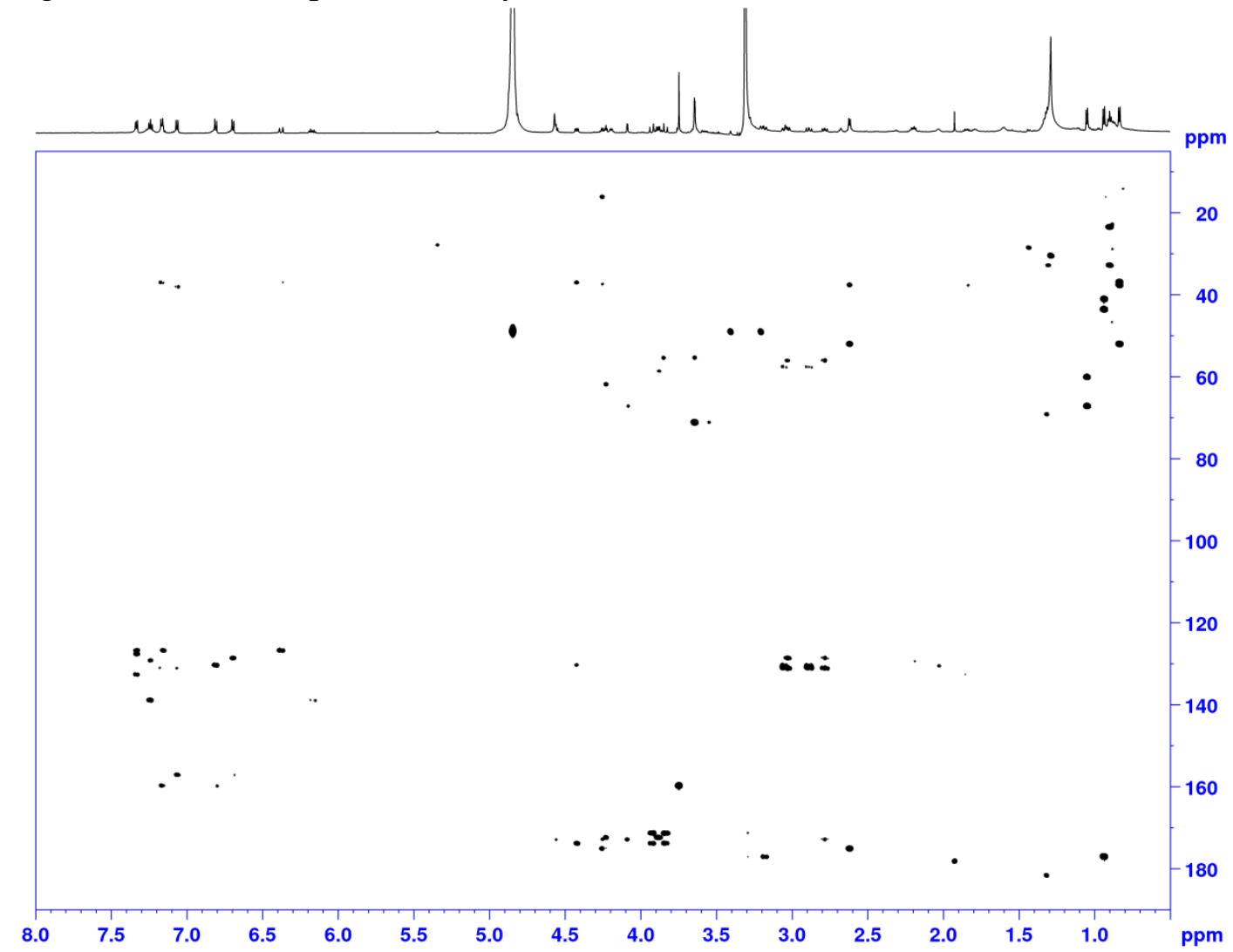

Figure S49: HMBC spectrum of Cyc-Thaxteramide A $\left(\mathrm{CD}_{3} \mathrm{OD}, 700 \mathrm{MHz}\right)$ 


\section{Cyc-Thaxteramide B}
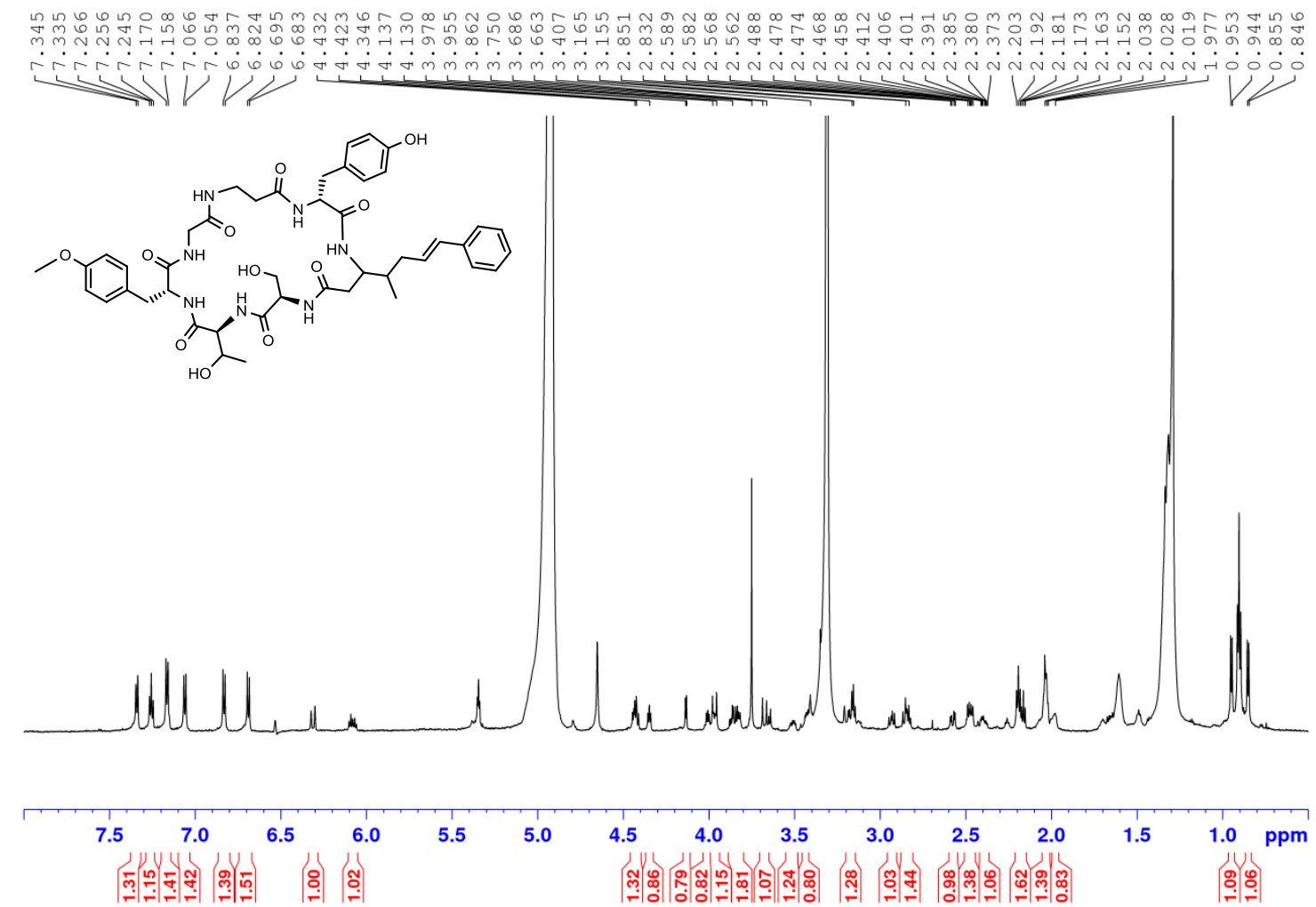

Figure S50: ${ }^{1} \mathrm{H}$ spectrum of Cyc-Thaxteramide $\mathrm{B}\left(\mathrm{CD}_{3} \mathrm{OD}, 700 \mathrm{MHz}\right)$

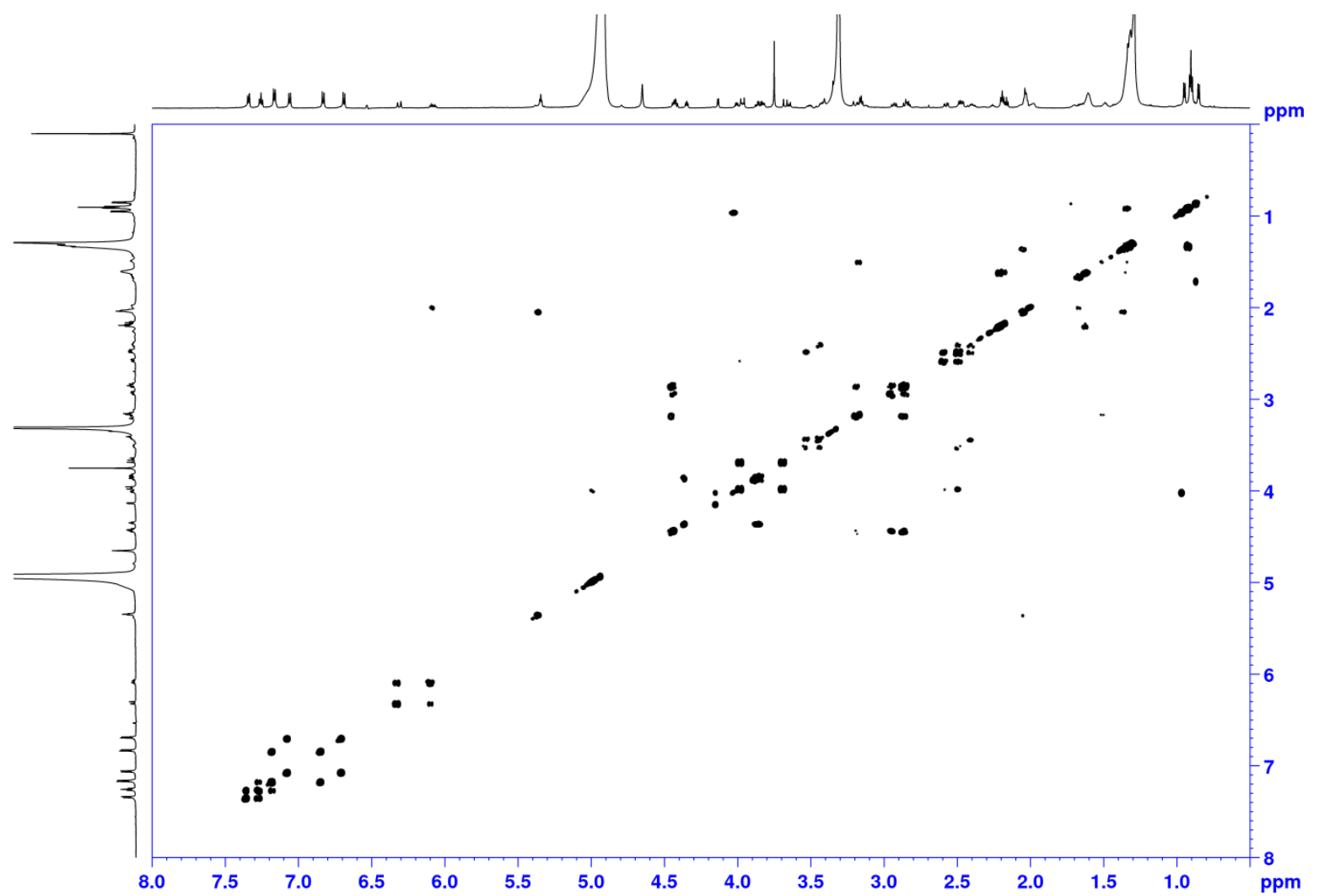

Figure S51: COSY spectrum of Cyc-Thaxteramide $\mathrm{B}\left(\mathrm{CD}_{3} \mathrm{OD}, 700 \mathrm{MHz}\right)$ 


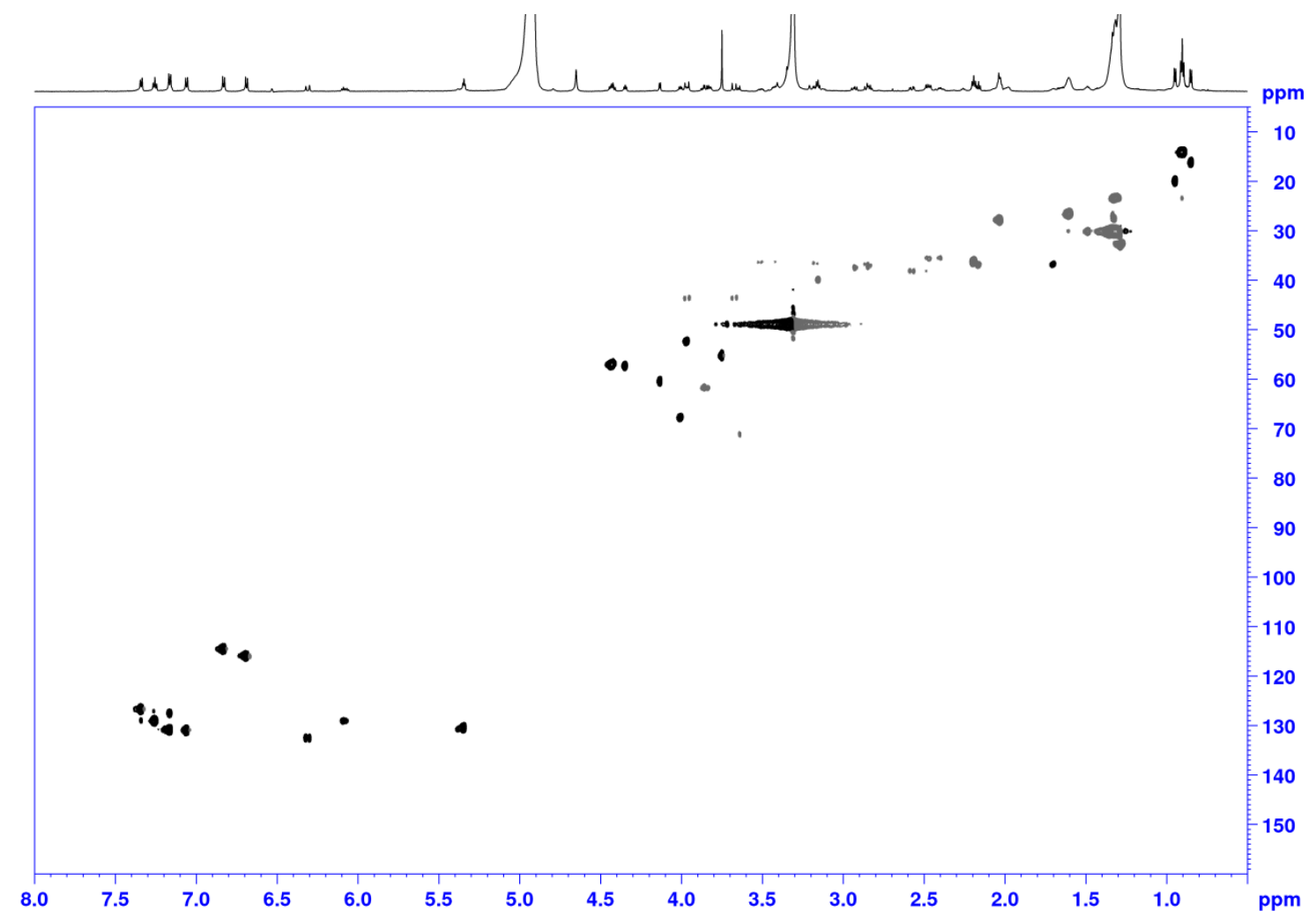

Figure S52: HSQC spectrum of Cyc-Thaxteramide B ( $\left.\mathrm{CD}_{3} \mathrm{OD}, 700 \mathrm{MHz}\right)$

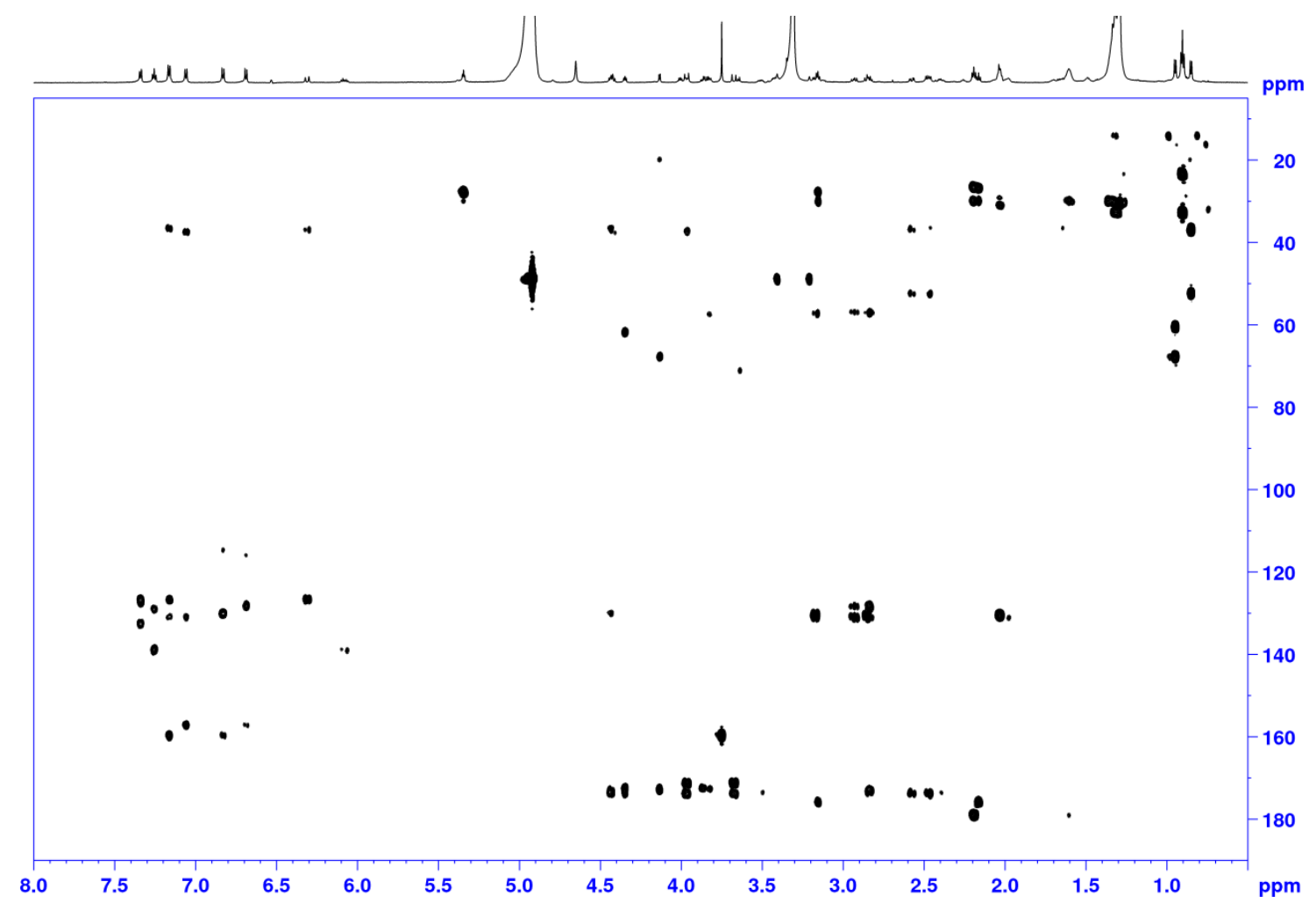

Figure S53: HMBC spectrum of Cyc-Thaxteramide B $\left(\mathrm{CD}_{3} \mathrm{OD}, 700 \mathrm{MHz}\right)$ 


\section{Cyc-Thaxteramide C}

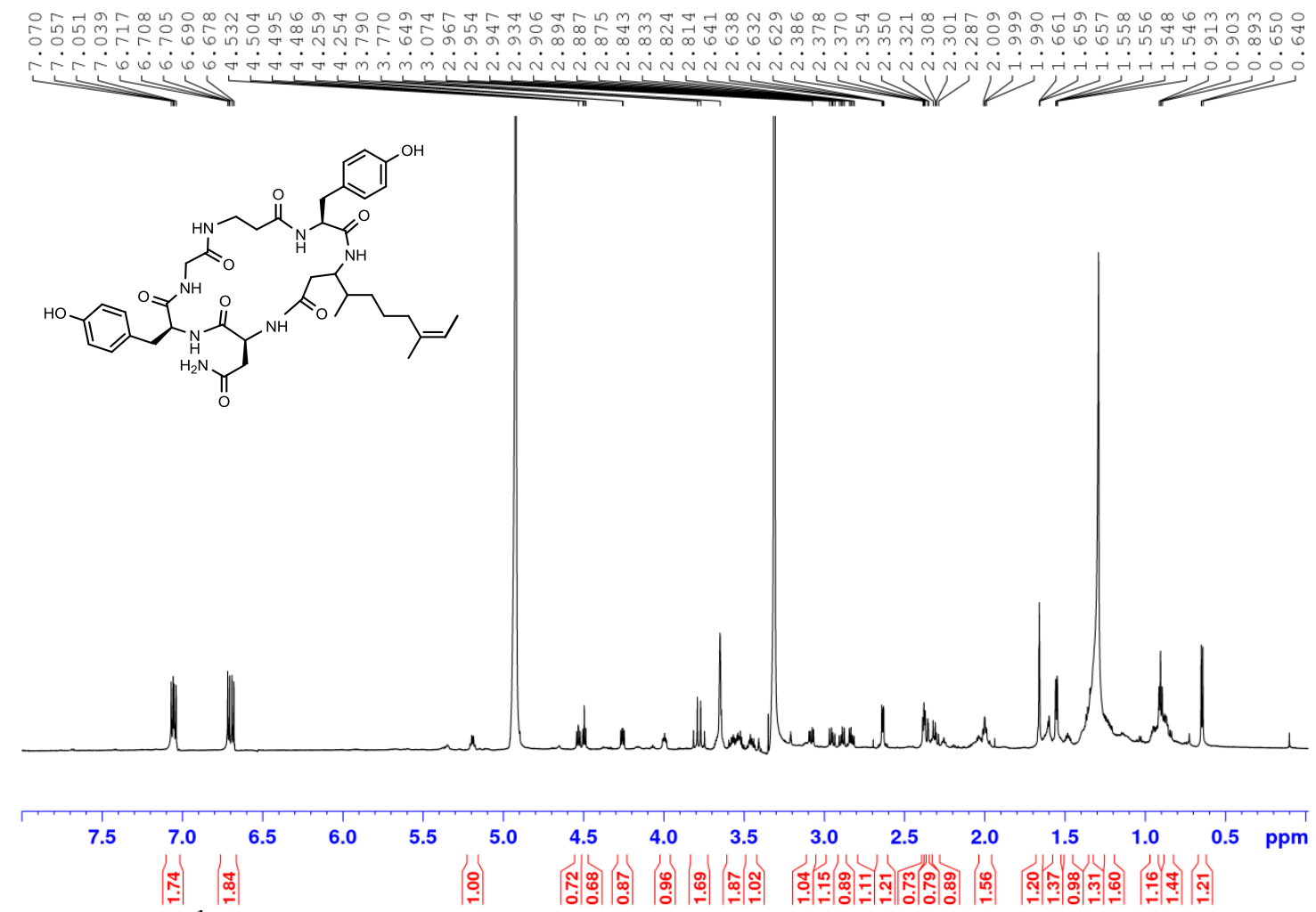

Figure S54: ${ }^{1} \mathrm{H}$ spectrum of Cyc-Thaxteramide $\mathrm{C}\left(\mathrm{CD}_{3} \mathrm{OD}, 700 \mathrm{MHz}\right)$

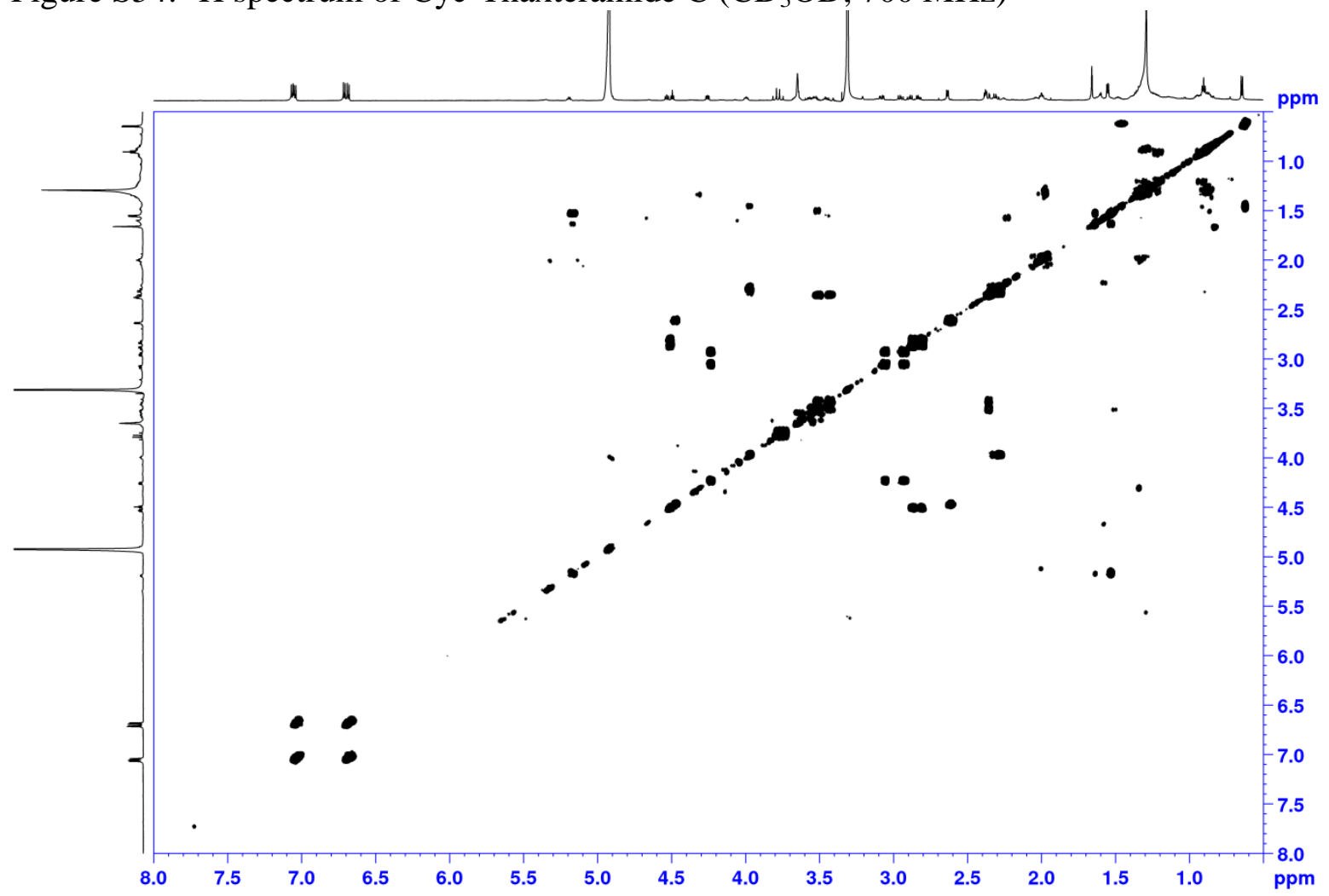

Figure S55: COSY spectrum of Cyc-Thaxteramide $\mathrm{C}\left(\mathrm{CD}_{3} \mathrm{OD}, 700 \mathrm{MHz}\right)$ 


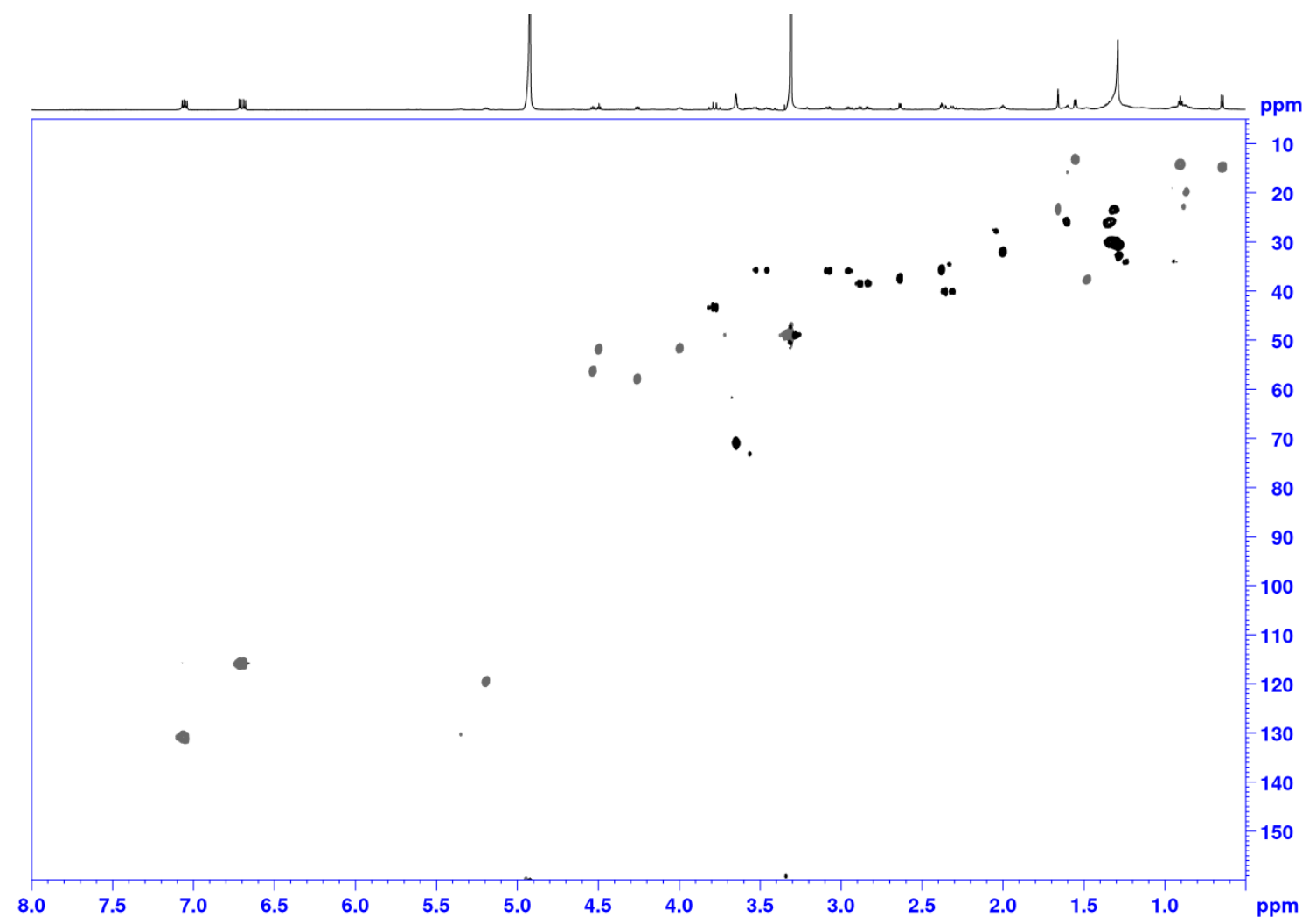

Figure S56: HSQC spectrum of Cyc-Thaxteramide C ( $\left.\mathrm{CD}_{3} \mathrm{OD}, 700 \mathrm{MHz}\right)$

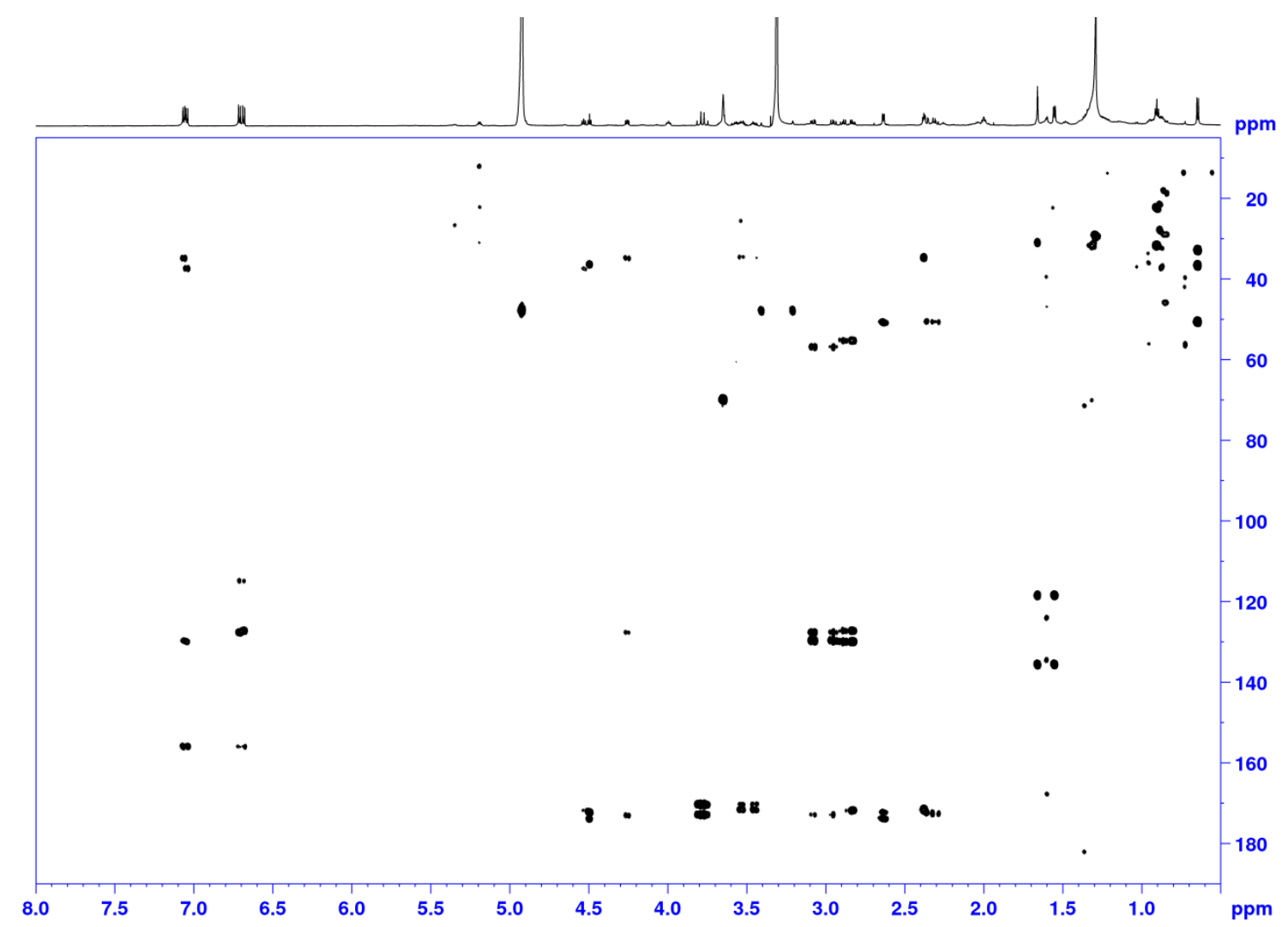

Figure S57: HMBC spectrum of Cyc-Thaxteramide C $\left(\mathrm{CD}_{3} \mathrm{OD}, 700 \mathrm{MHz}\right)$ 


\section{LCMS chromatograms and MS spectra}

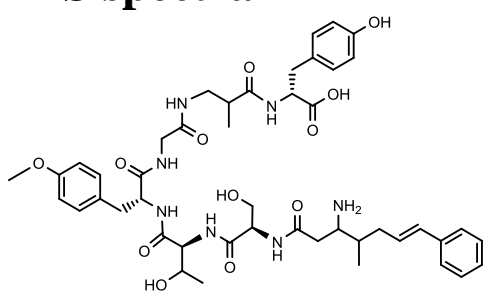

Thaxteramide A1 (ThxA1)

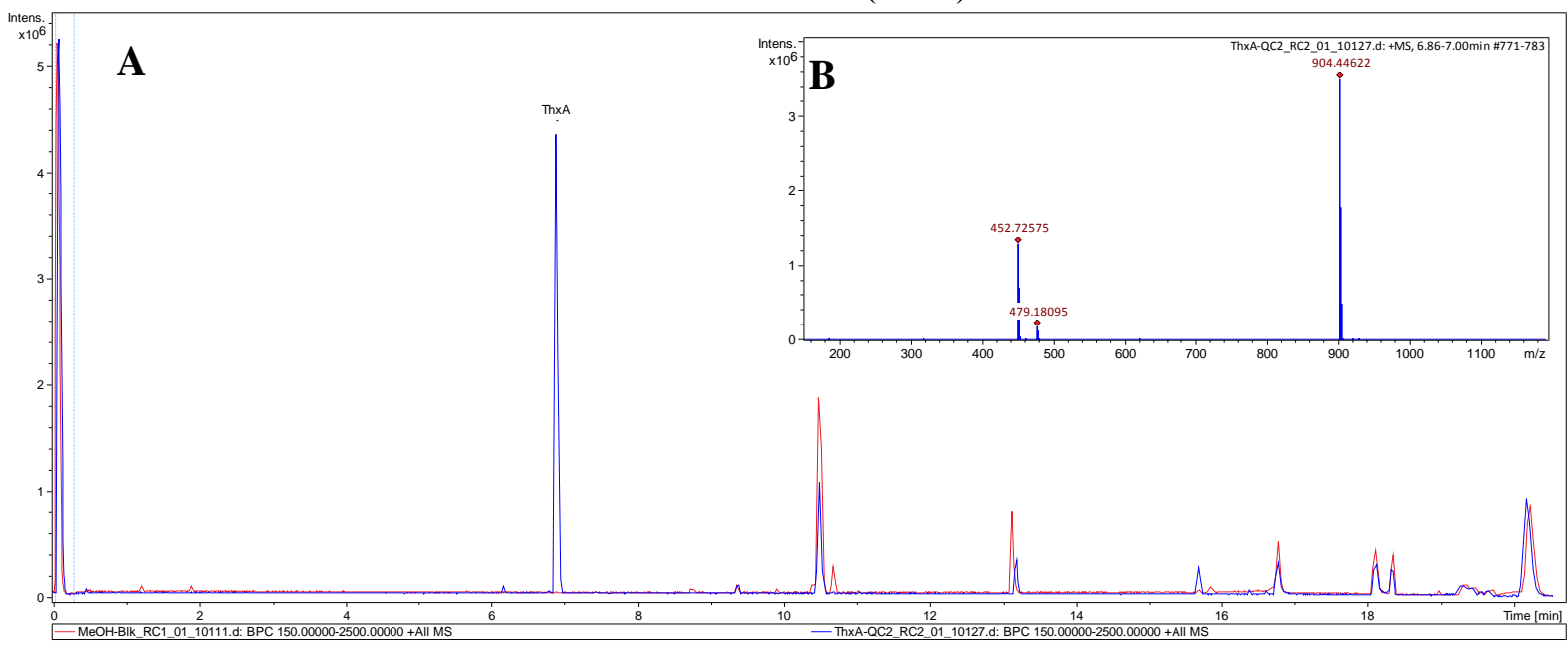

Figure S58: A- LCMS chromatogram of Thaxteramide A1 (Blue) and MeOH blank (Red); BHRMS spectra of ThxA1 peak; $m / z \quad[\mathrm{M}+\mathrm{H}]^{+}:$904.44622, $[\mathrm{M}+2 \mathrm{H}]^{2+} / 2: 452.72575$, $[\mathrm{M}+\mathrm{ACN}+2 \mathrm{H}]^{2+} / 2_{2}: 479.18095$.

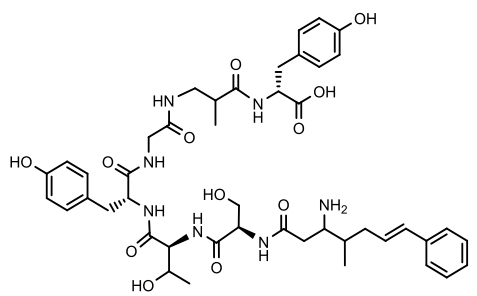

Thaxteramide A2 (ThxA2)

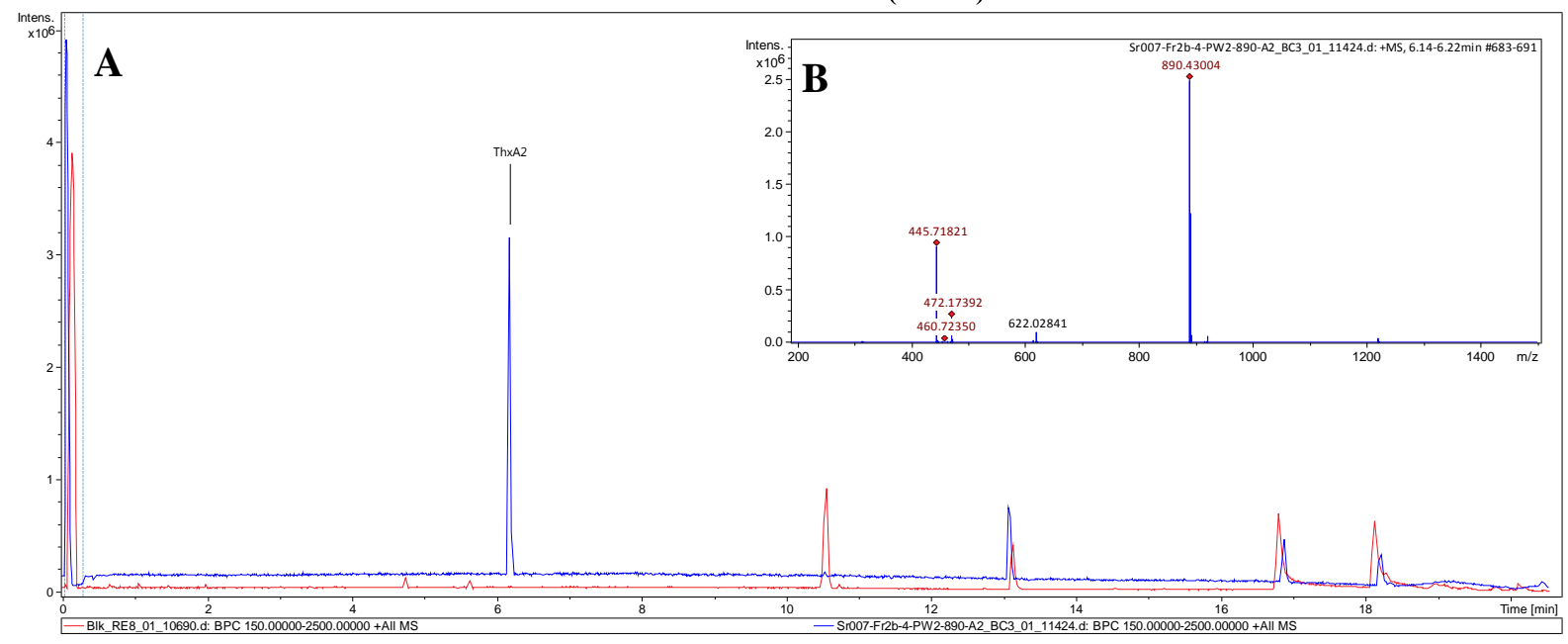

Figure S59: A- LCMS chromatogram of Thaxteramide A2 (Blue) and MeOH blank (Red); BHRMS spectra of ThxA2 peak; $m / z \quad[\mathrm{M}+\mathrm{H}]^{+}:$890.43004, $[\mathrm{M}+2 \mathrm{H}]^{2+} / 2$ : 445.71821, $[\mathrm{M}+\mathrm{ACN}+2 \mathrm{H}]^{2+} / 2: 472.17392$. 

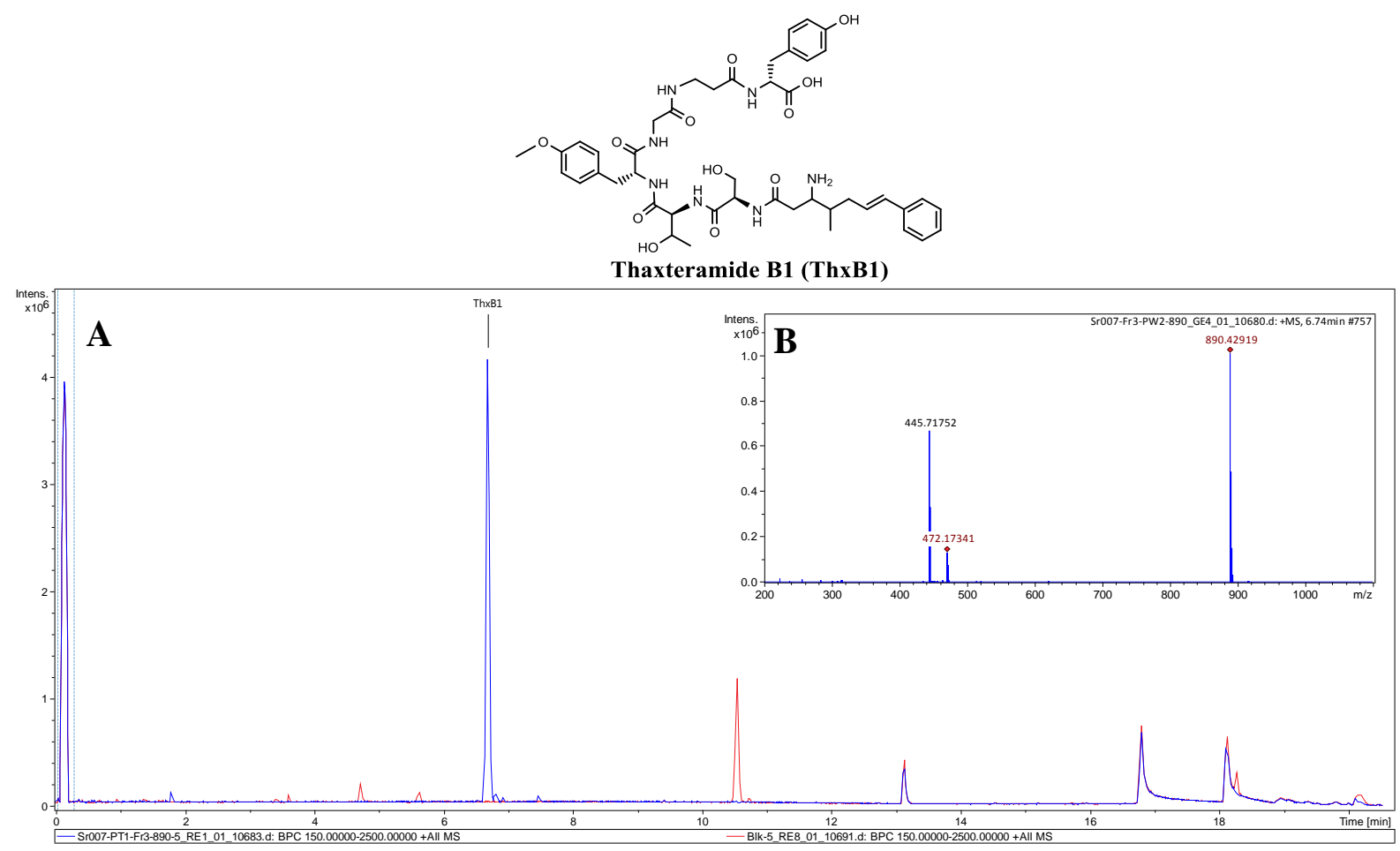

Figure S60: A- LCMS chromatogram of Thaxteramide B1 (Blue) and MeOH blank (Red); BHRMS spectra of ThxB1 peak; $m / z \quad[\mathrm{M}+\mathrm{H}]^{+}: \quad 890.42919, \quad[\mathrm{M}+2 \mathrm{H}]^{2+} / 2: \quad 445.71752$, $[\mathrm{M}+\mathrm{ACN}+2 \mathrm{H}]^{2+} /_{2}: 472.17341$.

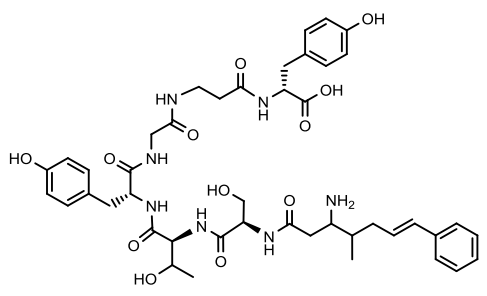

Thaxteramide B2 (ThxB2)

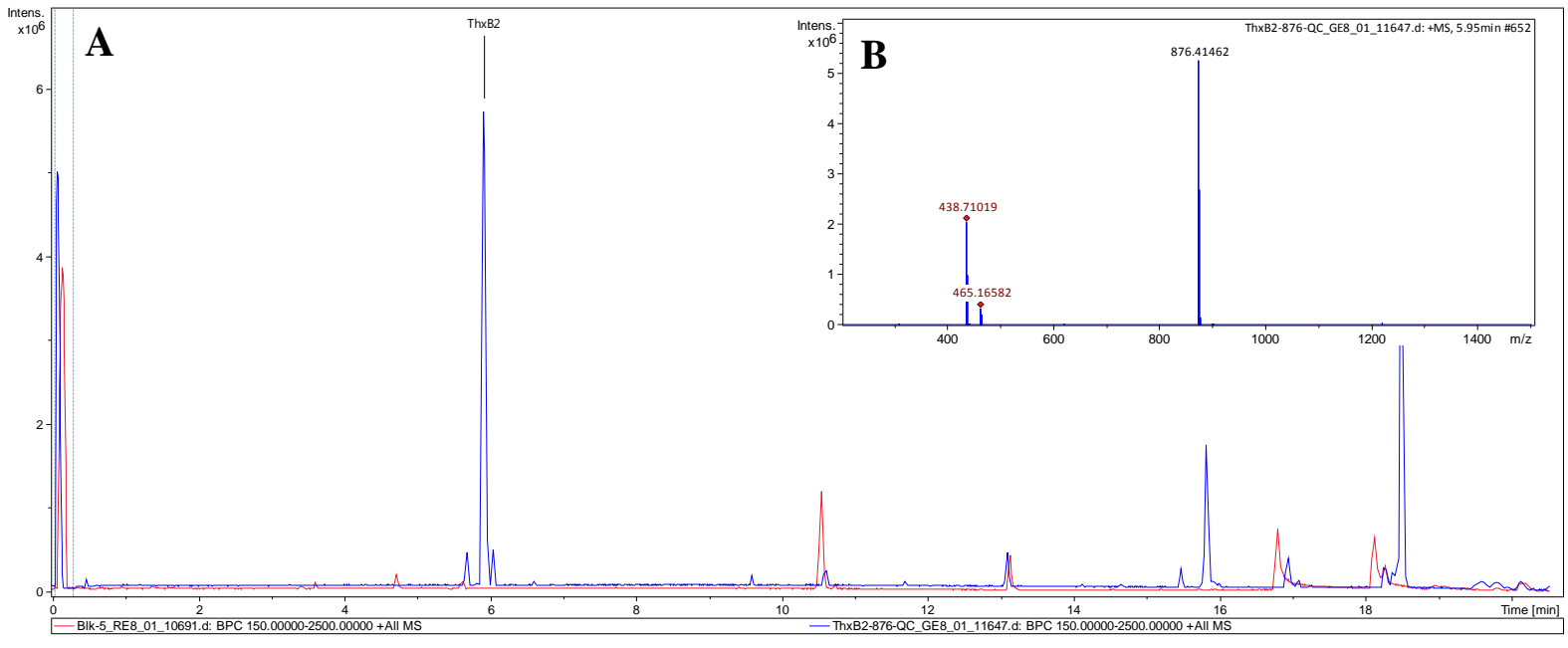

Figure S61: A- LCMS chromatogram of Thaxteramide B2 (Blue) and MeOH blank (Red); BHRMS spectra of ThxB2 peak; $m / z \quad[\mathrm{M}+\mathrm{H}]^{+}:$876.41462, $[\mathrm{M}+2 \mathrm{H}]^{2+} / 2$ : 438.71019, $[\mathrm{M}+\mathrm{ACN}+2 \mathrm{H}]^{2+} / 2_{2}: 465.16582$. 


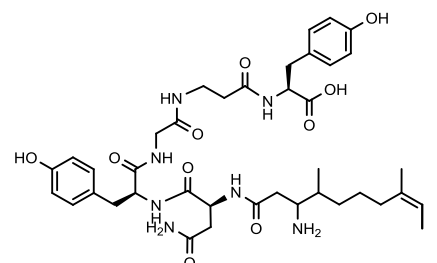

Thaxteramide C (ThxC)

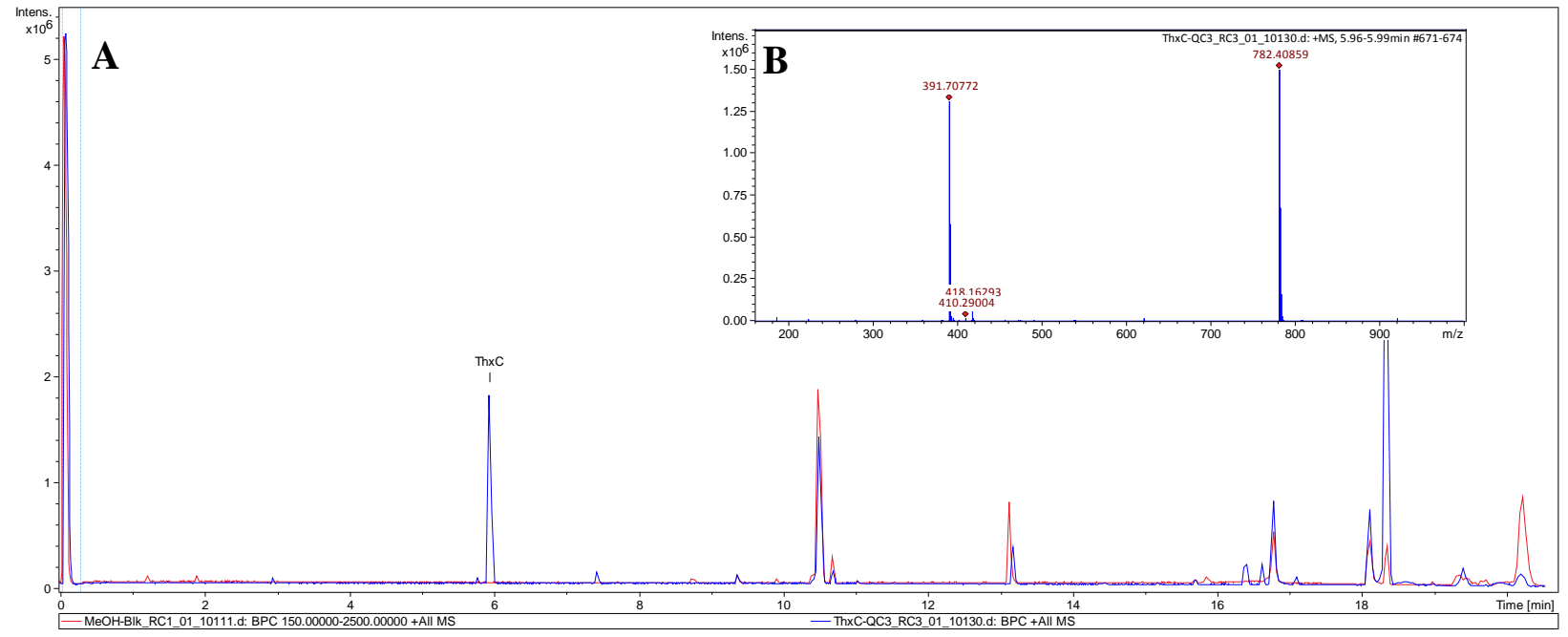

Figure S62: A- LCMS chromatogram of Thaxteramide C (Blue) and MeOH blank (Red); BHRMS spectra of ThxC peak; $m / z \quad[\mathrm{M}+\mathrm{H}]^{+}: \quad 782.40859, \quad[\mathrm{M}+2 \mathrm{H}]^{2+} / 2: \quad 391.70772$, $[\mathrm{M}+\mathrm{ACN}+2 \mathrm{H}]^{2+} / 2: 418.16293$.

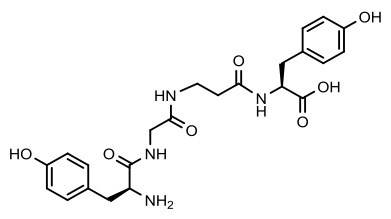

Thaxteramide D (ThxD)

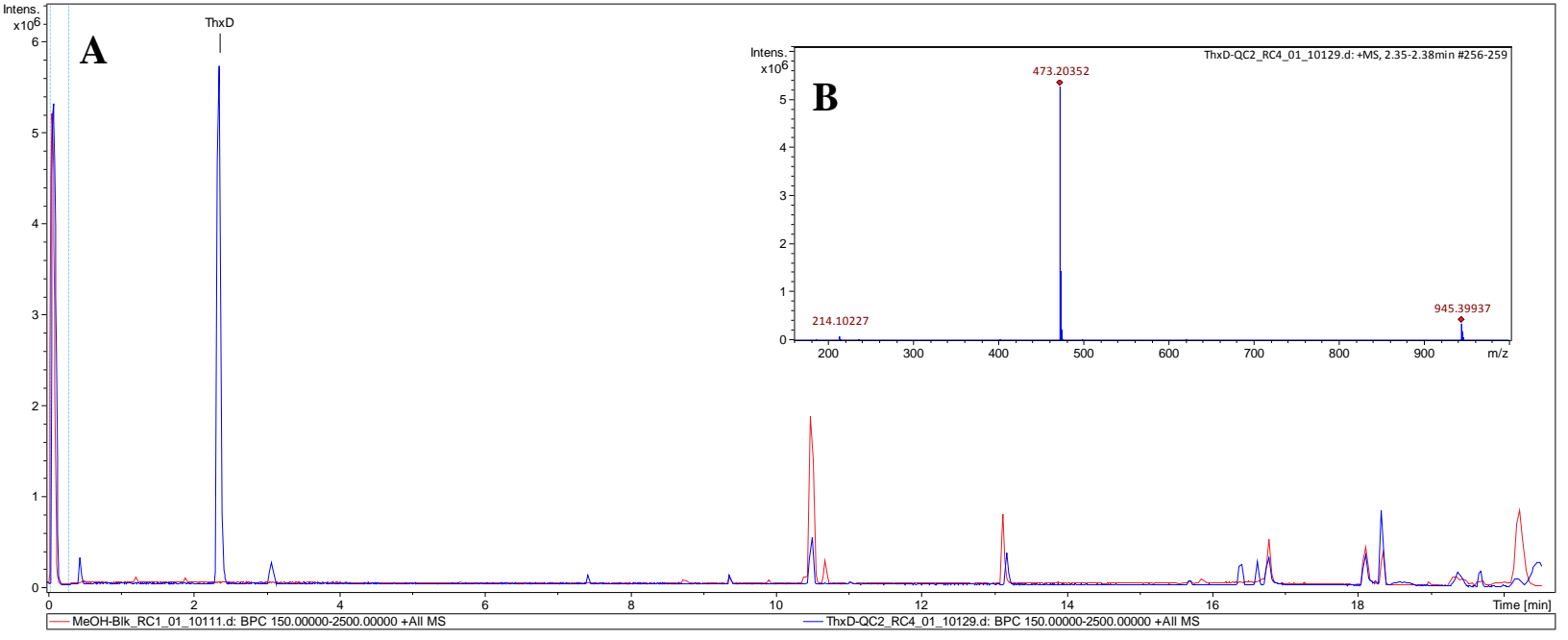

Figure S63: A- LCMS chromatogram of Thaxteramide D (Blue) and MeOH blank (Red); BHRMS spectra of ThxD peak; $m / z[\mathrm{M}+\mathrm{H}]^{+}: 473.20352,[2 \mathrm{M}+2]^{+}: 945.39937$. 

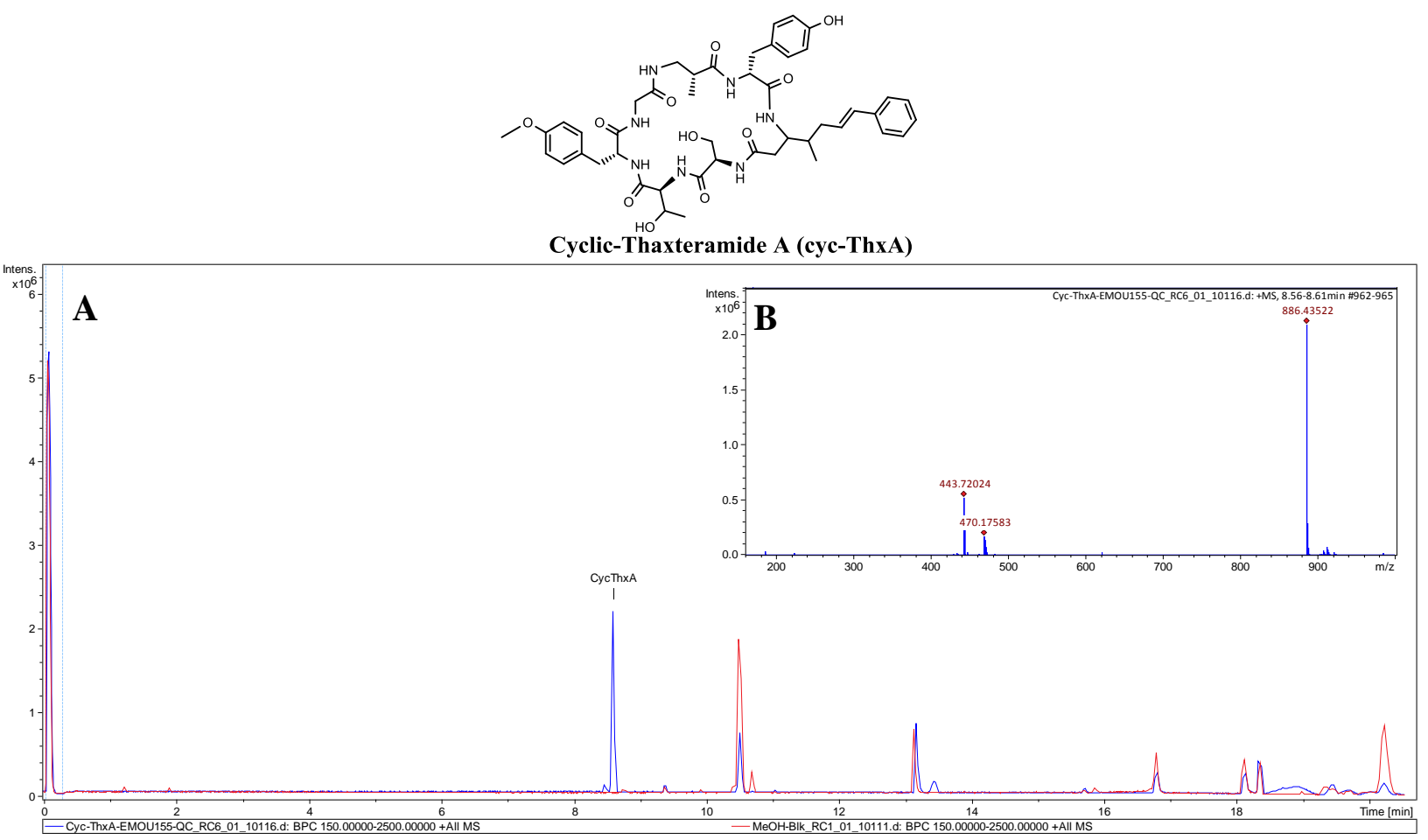

Figure S64: A- LCMS chromatogram of cyclic Thaxteramide A1 (Blue) and MeOH blank (Red); B- HRMS spectra of Cyc-ThxA1 peak; $m / z[\mathrm{M}+\mathrm{H}]^{+}:$886.43522, $[\mathrm{M}+2 \mathrm{H}]^{2+} /_{2}: 443.72024$, $[\mathrm{M}+\mathrm{ACN}+2 \mathrm{H}]^{2+} / 2: 470.17583$
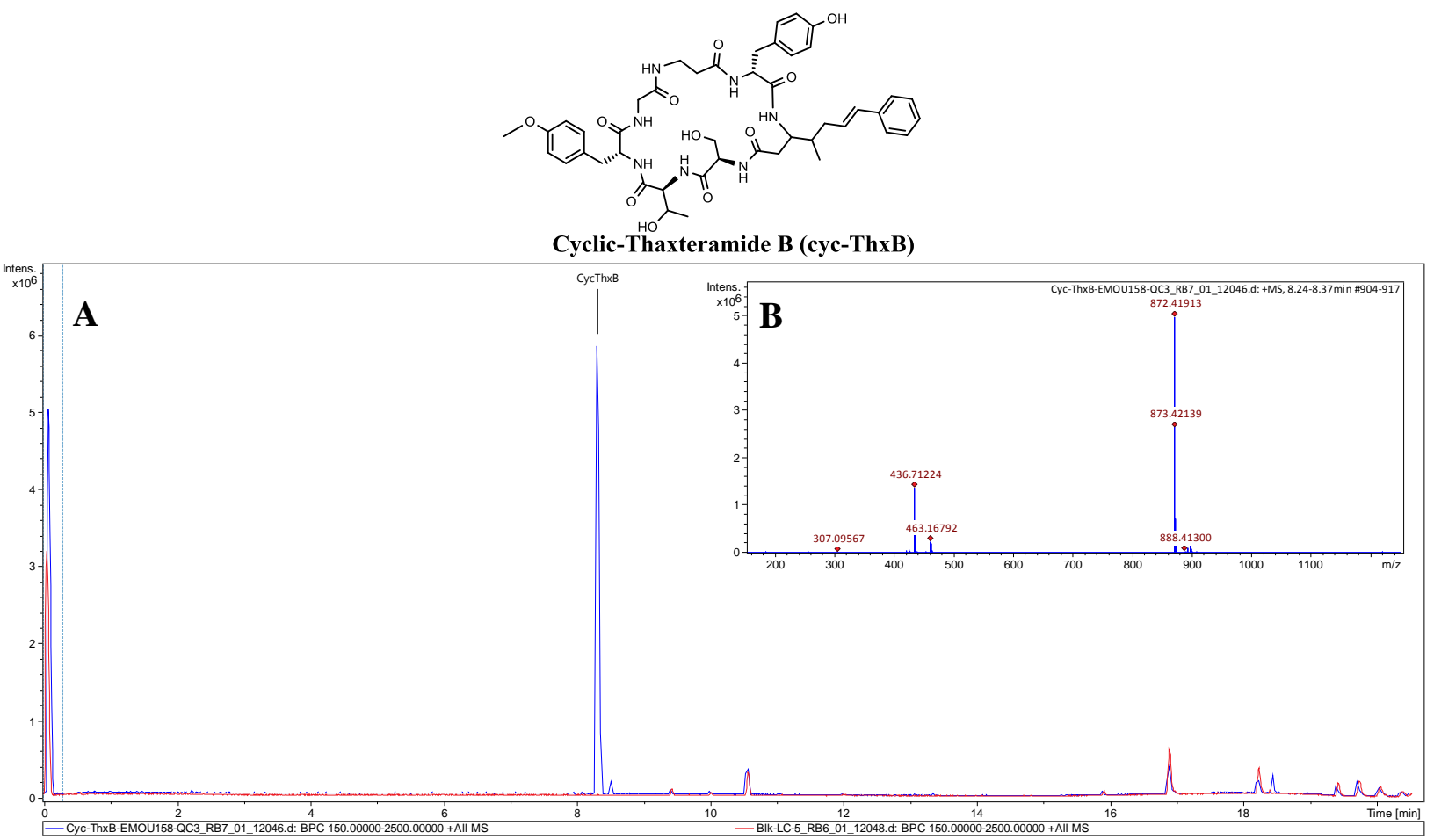

Figure S65: A- LCMS chromatogram of cyclic Thaxteramide B1 (Blue) and MeOH blank (Red); B- HRMS spectra of Cyc-ThxB1 peak; $m / z[\mathrm{M}+\mathrm{H}]^{+}: 872.41913,[\mathrm{M}+2 \mathrm{H}]^{2+}{ }_{2}: 436.71224$, $[\mathrm{M}+\mathrm{ACN}+2 \mathrm{H}]^{2+} /_{2}: 463.16792$. 

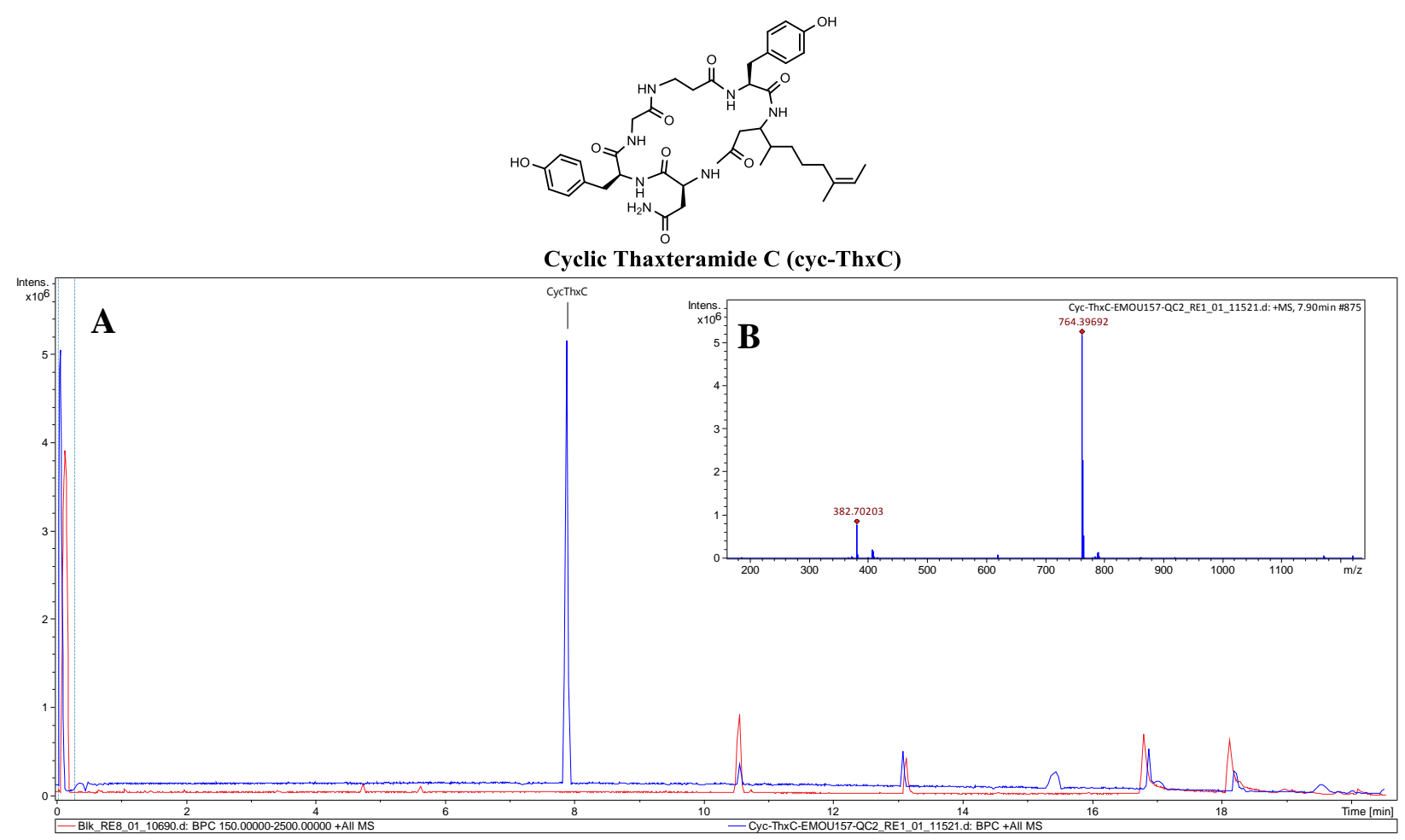

Figure S66 A- LCMS chromatogram of cyclic Thaxteramide C (Blue) and MeOH blank (Red); B- HRMS spectra of Cyc-ThxC peak; $m / z,[\mathrm{M}+\mathrm{H}]^{+}:$764.39692, $[\mathrm{M}+2 \mathrm{H}]^{2+} / 2:$ 382.70203, $[\mathrm{M}+\mathrm{ACN}+2 \mathrm{H}]^{2+} / 2: 409.15766$. 